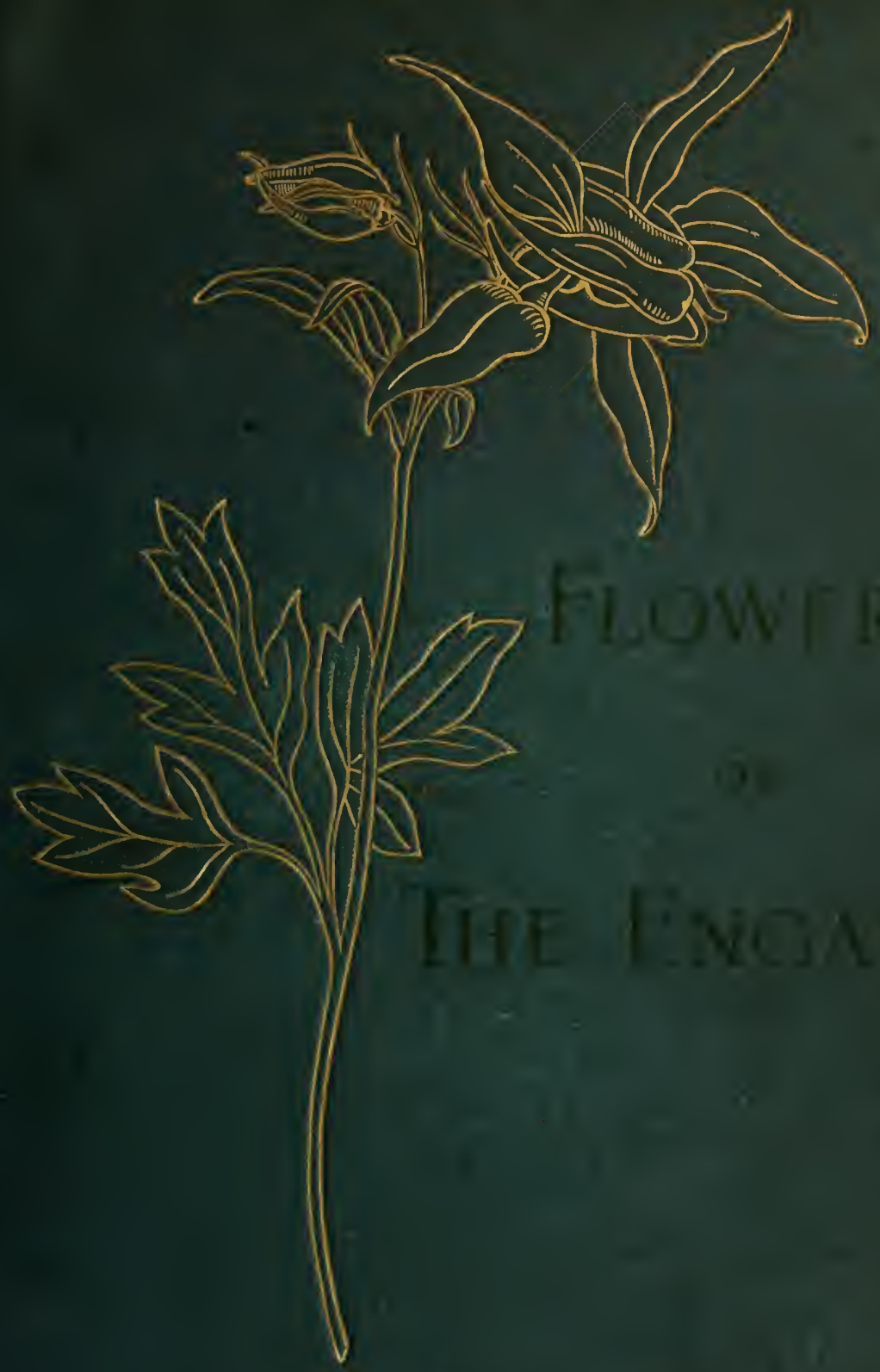




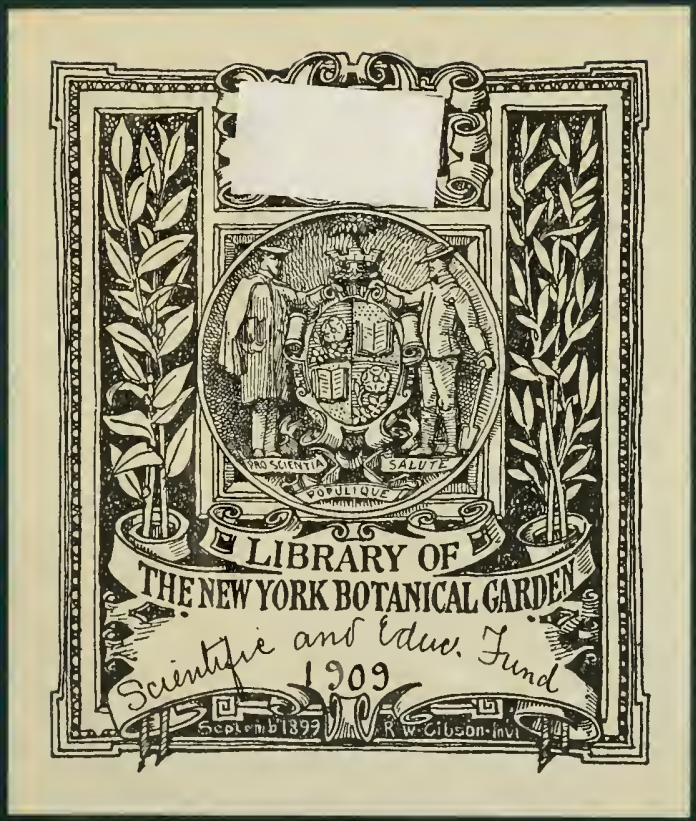




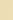


FL(NERS ()F THF ENGADINE. 



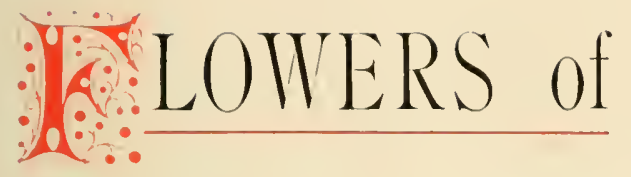

\section{THE NGADINE.}

\section{Drawn from illature}

EVELYN I), HEATHCOTE, M.A. 



\subsection{1:Naky \\ I i ioke \\ PRE II I C E. \\ - ivi at \\ ingerian}

$\mathrm{T}^{\mathrm{T}}$

II: following work has no higher aim than to assist lovers of flowers, who have but a slencler acquaintance with systematic botany, in naming correctly some of the plants they may find during a visit to the Engadine, and with this view more attention has been paid in the illustrations to the gencral appearance and habit of growth of the various plants, than to the details of their structure; at the same time, though the drawings might no doubt have been more elaborate, it is hoped they are accurate as far as they go.

Many people while in the Engadine are induced by the profusion and beauty of the wild flowers around them, and by the ease with which even the high growing alpine species are obtained, to begin for the first time a collection of dried plants, which they naturally wish to name correctly, but with only a small knowledge of botanical terms, they find the ordinary books difficult to understand and wish for some assistance in the form of illustration. Ten years ago, when these drawings were made, Weber's pretty little work in four volumes, containing plates of 400 flowers, was in frequent use, and probably still is: but some of his illustrations are misleading from being reduced in size without a notice to that effect, some give a very inadequate idea of the plant in question, while, as far as the Engadine is concemed, not far short of half the number of plates represent flowers which have not been found in that locality at all. The employment of the present volume outside the boundary of the valley it is especially intended to illustrate, would not labour under the same disadrantage, or at least not to the same extent, for there are very few plants growing in the Engadine which are not found in some other part of Switzerland as well, and the only ones represented in this book are, I believe, Primula glutinosa and P'rimula dinyana. I am aware that Dr. Taylor, in his Flowers: their origin, shapes, etc., says that "on the Engadine, a high valley in the Canton des Grisons, there are found eighty. species of flowering plants unknown to the rest of Switzerland, although they are very common in the extreme north of Europe," and he kindly informs tit me that the statement was made on the authority of a paper in the Rerue 之 des De'ux Monde's, in February, 1870, by Prof. C. Martin, but a reference to the article itself will 1 think prove that this is a mistaken inference from the words 
used. Prof. Martin, indeed, says that in the Engadine there are a certain number of species unknown to the rest of Switzerland, but very common in the north of Europe, and instances five: Thalictrum alpinum Trientalis europea, Juncus castaneus and stygius, Carex Vahlii; then he goes on to state that the number of "plantes boréales" amounts in the Engadine, according to M. Heer, to eighty, but says nothing about these being unknomn in other parts of Switzerland, and incleed the very instance he gives out of these eighty, "Le Saule des Lapons" proves this, for it is a plant which is found in other parts of the Swiss Alps. Even of the five species given by Prof. Martin two at least appear to have been found elsewhere: Trientalis europaea in Ursernthale and Juncus stygius in Canton Schwyz, near Einsiedeln.

It must not be imagined that the present volume pretends to be a complete "Jlora of the Engadine," or even contains drawings of all the rarer species,-it can only be considered as a contribution to such a work; but the illustrations have this advantage, that the originals were all drawn and coloured from freshly. gathered specimens, and are of the size of life. The plates are arranged according to Bouvier's Flore des Alpes de la Suisse et de la Savoie, 1878; and in the short descriptive catalogue, I have made frequent use of Wood's Tourist's Flord. The English, French, German, and Italian names of the Genera are given where they are not a mere reproduction of the Latin ; also a sign of duration, or of general size, as a, for annual, T, for tree; the approximate months of flowering expressed in figures; the letter $B$ if the plant is an inhabitant of the lritish Isles; the position, or soil in which any plant may be expected to be found; any peculiarity which serves to distinguish one flower from another bearing a general resemblance to it ; and one locality for all the rarer species. With regard to this last point, it will be understood that the plants are not confined to the localities named, but that I have gathered them myself in the spots so given in every case, except where the letter $\mathrm{C}$ follows the locality, when I have the authority of Herr Caviezel, of Pontresina, that they grow there. My thanks are in an especial manner due to him, and to his two sons, who brought me specimens of many of the rarer species, and gave me much useful information, without which this volume would have been more imperfect than it is.

\section{EVELYN D. HEATHCOTE.}




\section{AIBREVIATIONS.}

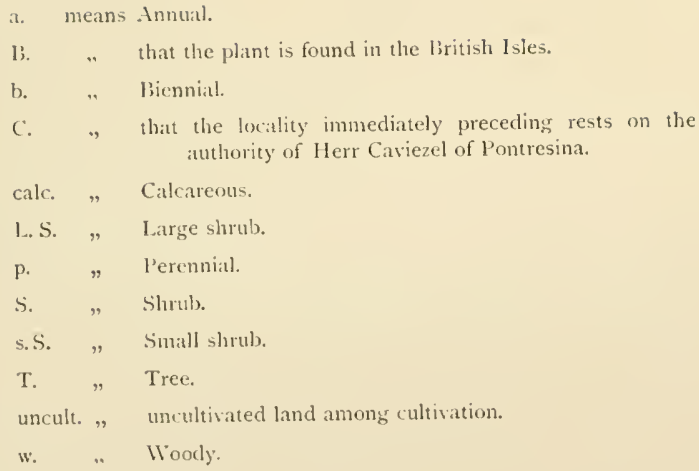




\section{Jflowers of the Engadinc.}

\section{DICOTYLEDONES.}

THALAMIFLORA.

I.-RA NUNCULACE $Æ$ Juss.

1. Clematinez.

Atrage'ne 1.... Ilpenrebe, Gor.

Polyandria, Polygyia, L. Plate alpina L. W. 6 - 8 . Rocky woods.

Alp Surlej. I.

\section{2.-ANEMONEE.}

Thali'ctrum I.-Meadow Rue. Pigamon, Fr. Wiesenraute, Gcr. Polyandria, Polygynia, L.

aquilegifólium L. p. $6-8$. Jountain woods. Rosegthal. n. minus I. p. 7-8. B. Hills and dry pastures.

Silvaplana. Iи.

Anemo'ne L. - Windfower. Windröschen, Ger.

Polyandria, Polygynia, L.

vernális L. p. $5-7$. Pastures and rough grass.

Pontresina. w.

alpina 1. p. $6-7$. (irassy hili sides.

Schafberg. v.

alpina 1. var. sulphirea, p. $6-7$. Grassy hill sides.

Silvaplana. v.

\section{3.-R.NUNCLIE.}

Ranu'nculus 1.-Crowfoot and Buttercup. Hahnenfuss, Ger.

Polyandria, Polygynia, $L$.

capillaceus Thuill. p. $6-8$. B. Water.

Pontresina. vi.

glacialis I. p. :- -8. Stony, near snow, often rose coloured. Cambrena.

v1.

alpéstris I.. p. 6 - S. High.

Julier. vi.

aconitifólius I. p. $6-8$. Woody mountains.

Rosegthal. vis.

pyrena'us L. p. $6-7$. High moist.

Sils. vIII.

parnassifólius I., p. 6 S. Near snow.

Albula. C. vir.

Thóra I. p. 6- 7 . High pastures; no root leaves.

Aibula. C. Ix.

hy'bridus Bir. P. 6-7. High calc: stony; root leaves.

Flámmula 1. var. réptans, p. 7 9. H. Wet.

Albula. C. Ix.

Campfêr. Ix.

montánus Willd. p. 5-7. Pastures, etc. Receptacle hairy.

répens I. p. 6-8. B. Noist meadows.

Pontresina. $x$.

Pontresina. $\mathrm{x}$. 


\section{4.-HeLlebore..}

Ca'1tha L. Marsh Marigold. Populage, Fr. Dotterblume, Ger. Sposa del sole, It. Polvandrit, Polıgynia, L.

palístris L. p. 5-7. B. Marshy.

common. xil.

Tro'llius L.-Globe-Flower. Kugelranunkel, Ger.

Polyandria, Polygyiui, L.

europre'us L. p. 6-7. B. Mountain pastures; open woods. common. xirr.

Aquile'gia L.-Columbine. Ancolie, Fr: Akelei, Ger:

Polyandria, Oligogynia, L.

vulgaris L. var. atrata, p. $6-7$. Shade: stamens exceeding the very dark flower. Sils.

alpina L. p. $7-8$. High stony; edges of woods. Rosegthal.

xiv.

xx.

Aconi'tum L.-Wolf's-bane, Monk's-hood. Eisenhut, Ger.

Polyandria, Oligoginia, $L$.

I.jcóctonum L. p. 7-S. Mountain woods.

common. XY1.

Napéllus L. p. 7 -8. B. Woods; pastures; open stony: paniculátum Lam. p. $7-8$. Mountain woods.

common. xvil.

Rosegthal. xint.

\section{II.-BERBERIDEA Tent.}

Be'rberis L.-Barberry: Vinettier, Fr. Sauerdorn, Ger. Crespino, It. Hexandria, Monogyiar, $L$.

vulgáris L. S. $6-7$. R. Stony.

Pontresina.

xix.

III. PAPAVERACEA Juss.

Papa'ver L.-Poppy. Pavot, Fr. Mohn, Ger.

Polyandria, Monogyia, L.

alpinum L. var. flaviflórum, p. 6-8. High stony.

Cambrena. xix.

\section{IV.-CRUCIFERA JusS. \\ Tetradyania L. \\ SILIQUOSAE.}

1.-AKABIDE.E.

A'rabis L.-Wall or Rock Cress. Arabette, Fr: Gänsekraut, Ger. cærílea Hænk. p. 7-8. High rocky.

bellidifólia Jacq. p. 6-8. Moist.

Bernina. C. $\mathrm{x} 1 \mathrm{x}$.

alpina L. p. $5-8$. Stony.

Stätzer-See. $\mathrm{xx}$.

Halléri L. p. 5-7. Stony grass.

Pontresina. $\mathrm{xx}$.

alpéstris Reichb. var. ciliáta, b. 6-7. Dry.

Pontresina. $\mathrm{xx}$.

Pontresina. $\mathrm{xx}$.

Cardami'ne L.-Lady's Smock, Bittercress. Schaumkraut, Ger. Billeri, It. a) pína IVilld. p. 7 - . High.

resedifólia L. a. b. or p. 6-8. Stony.

amára L. p. 6-7. B. Wet. Anthers violet.

praténsis L. p. 6. B. Moist. Anthers yellow.

Bernina. $\mathrm{xx}$.

common. xxi.

Pontresina. xxı.

Stätzer-See. $\mathrm{xx}$. 
Nastu rtium R. Br. Cress. ('resson, fir. Brunnenkresse, Gir. Cres cione, $I l$.

paluistre 1) C. b. 6-9. B. Wet.

Samaden. C. $\mathrm{xxu1.}$

2. -ISYMBRIE.

Ery'simum L.--Treacle-mustard. Vélar, Fr. Hederich, Ger: Crespinaccio. $1 t$.

rha'ticum DC: 1., 6-7. Rough hill sides.

Silvaplana. xxı.

\section{S I L I C U LOSAE.}

\section{It.Msink.}

Dra'ba 1,. Whitlow-grass. Drave, Fr: Hungerlliumchen, fier. aizoides L. p. 5 - 6. B. Open rocks. St. Moritz. C. xxv. tomentósa Wahl. var. frigida, [. 5 i. Rocks and high pastures.

Pontresina. xxv.

Cochlea'ria L.--Scurvy-grass. Cranson, Fr. Löfelkraut, Ger. saxitilis Lam. p. 6-8. Kocks and stony.

Bernina road. xxy.

Hutchi'nsia R. Br.

$$
\text { 4.-Thlaspine. }
$$

alpina K. Br. p. $6-8$. High stony.

Bernina. $\mathrm{xxv}$.

Biscute'lla 1.--Buckler-mustard. Lunetière, Fr: Brillenschote, Ger: lavigáta L. p. $6--8$. Rocks and stony pastures.

Silvaplana. $\quad \mathrm{xxv1.}$

\section{V.- CISTINE E Juss.}

Helia nthemum Tourn.-Rock-rose. Sonnenröschen, Ger.

Polyandria, Monogynia, $L$.

vulgaire Gartn. w, 6--. B. Dry pastures; with stipules. Pontresina. xxvin.

celándicum Wahl. W. 7-S. Stony; no stipules. Morteratsch. xxvit.

\section{VIOLARIEA DC.}

Viola L. - Violet. Violette, Fr. Veilchen, Ger.

pinnata L. P. 6 - 7. Pastures. palistris L. p. $5-7$. B. Moist.

Thomasiána Perr. et song. p. 6. Stony.

canina L. p. $6-7$. l3. Woods and open.

mirábilis L. 1) 6. Woods.

biflóra L. P. 5 S. Moist rocky:

tricolor L. var, alpestris, a, b, $6-9$. Fields, etc. calcaráta L. p. 6 -8. Woods and pastures.

calcarata L. var. flavia, 1. 6-8. Pastures.
Pentandria, Jonogrnia, $L$.

$\begin{aligned} \text { Samaden. C. } & \text { xxvun. } \\ \text { Fex. } & \text { xxvın. } \\ \text { Languard. } & \text { xxıx. } \\ \text { l'ontresina. } & \text { xxvı. } \\ \text { Celerina. C. } & \text { xxx. } \\ \text { common. } & \text { xxxı. } \\ \text { common. } & \text { xxxı. } \\ \text { Stätrer-See. } & \text { xxxı. } \\ \text { Sils. C. } & \text { xxxı. }\end{aligned}$




\section{VII.-DROSERACEÆ DC.}

Dro'sera 1.--Sundew. Rossolis, Fr. Sonnenthau, Gcr. Ruggiada del sole, It.

Pentendria, Pentaginia, L.

rotundifólia I. p. 7 -8. B. Bogs; leaf stalks hairy.

Flatz-bach. xxxil.

longifólia 1. p. 7 - 8. B. Bogs; leaf stalks hairless.

Stätzer-See. xxxil.

Parna'ssia L. - Grass of Parnassus. Einblatt, Ger.

Pentandria, Tetraginia, L.

palústris L. p. 7 -9. B. Moist.

Silvaplana. xxxir.

VIII. POLYGALE $Æ$ Juss.

Poly'gala L.-Milkwort. Kreutzblume, Gir. Diadelfhita, Octandria, $L$. atmára I. p. $6-8$. B. Hills, dry.

Chamebúxus L. w. $6-7$. Woods and stony:

Rosegthal. xxxıl.

Campfêr. xxxill.

IX.-CARYOPHYLLEÆ JUSS.

\section{I.-SILFNE.E.}

Dianthus L.-Pink. (Eillet, Fr: Nelke, Ger. Garofano, It.

Deandria, Digyin, $L$.

atrorúbens Koch. p. 7-8. Grassy. Bracts coriaceous.

Sils. xxxin.

deltoídes L. p. 7-8. B. Stony pastures.

Samaden. xxxil.

* sylvéstris Wulf. p. 6-9. Rocky.

common. xxxiv.

supérbus L. p. b. 7 -8. Stony.

Gypso'phila L.--Gipskraut, Ger.

répens L. p. 6-8. Stony.

Dicandria, Digynia, $L$.

Surlej. xxxuv.

Sapona'ria L.-Soapwort. Seifenkraut, Ger. Detandria, Digynia, L. ocymoídes L. p. 7-8. Stony.

Sile'ne L.-Catchfly or Campion. Cornillet, Fr. Leimkraut, Ger:

Samaden. xxxy.

Decandria, Trigynia, L.

infláta Sm. p. $6-8$. B. Fields, etc.

Pontresina. xxxr.

† Arméria L. a. 7 -9. Stony.

Brusio. C. xxxy'

rupéstris L. p. $6-8$. J)y.

Pontresina. xxxvit.

acaúlis L. p. $6-7$. B. Stony.

Morteratsch. xxxvit.

nútans L. p. 6-8. B. Rocks and pastures.

Mela'ndrium Rohl.-Catchfly and Campion.

Silvaplana. xxxviı. sylvéstre Roehl. p. 6-8. B. Fields, etc.

Diocia, Decandria, $L$.

Pontresina. xxxix.

* Usually said to be scentless, but in the Engadine generally sweet scented. Mr. F. N. Williams, F.1. ५., tolls me that he has found scented and scentless specimens growing in adjacent localities in Tyrol.

+ This plant was admitted into this collection in consequence of a mistake as to the position of Brusio, which though in the Grisons, is in Val Poschiavo, and therefore not in the Engadine. 
Ly'chnis L. - Campion. Lichtnelke, Gir: Decandria, Pentagynia, L.

alpína 1. p. 7-8. 13. High.

lilos-Cúculi L. P. 7. 1. Moist meadows.

Javirun. C. $\mathrm{xL}$.

Flos-Jóvis lam. p. 7 . Open stony.

Silvaplana. $\mathrm{xL}$.

Bevers. C. XLI,

\section{2. -AISINF, \%.}

Spergula'ria l'ers.--Sandspurrey:

Decandriu, Triginia, l.

rúbra Pers, a. 7 - 8. B. Dry; roadsides.

Pontresina. xLII.

Sagi'na L.-Pearlwort. Vierling, Ge'r. Decundria, Pentugynia, L. linna'i Presl. b. 6 -8. B. 1)ry.

Pontresina. xL11.

Alsi'ne Wahl. --Sandwort.

Decandria, Trigluia, L.

laricifólia Crantz. p. 7 8. 1)ry pastures.

Silvaplana. XLn.

vérna liartl. p. $6-8$. B. Stony.

common. XL11.

Cherléria Fenzl. p. 7 - 8. 13. High.

liernina. XLıII.

Arena'ria L.-Sandwort. Sabline, Fr: Sandkraut, Ger.

Deiandrin, Trigynia, L.

cilinita 1. p. $7-8$. B. High stony : sepals $3-5$ nerved.

Albula hospitz. C. xull.

biflóra L. p. 7-8. High ; sepals one nerved.

Bernina. xull,

Stella'ria L.-Stitchwort. Sternmiere, Ger. Decandria, Trigynia, L.

némorum L. p. 6 -7. l3. Moist woods.

Campfer. xulv.

Cera'stium 1.-Mouse-ear Chickweed. Hornkraut, Ger.

Decandria, P'entagymia, $L$.

trigy'num Vill. p. 6-8. 13. Moist ; styles $3-5$.

Pontresina. xis.

alpinum I. p. 7 - S. B. High stony:

Cambrena. xur.

arvínse l. p. 5-8. I3. Open hills and banks.

common. XLV.

\section{HYPERICINE死 JusS.}

Hypericum L.--St. John's Wort. Millepertuis, Fr. Johanniskraut, Ger. Pilatro, $I t$.

Polyadelphia, Polyandria, L.

quadrángulum L. 1). 7 -8. 13. Moist.

Silvaplana. xLv.

\section{XI.-GERANIACE $Æ$ Juss.}

Gera'nium L.--Crane's-bill. Storchenschnabel, Ger:

Ilonadelphia, Decandria, $L$.

sylváticum J. p. $6-8$. B. Woods and meadows.

common. XLvH.

aconitifólium L'Herit. p. $6-8$. High.

Rosegthal. XLVIII.

\section{OXALIDEA DC.}

O'xalis L.-Wood Sorrel. Surelle, Fr. Sauerklee, Ger. Alleluia, It. Decundrit, Pentageniu, $L$.

Acetosélla 1. p. 6 - 7. B. Moist shade.

l'ontresina. xLIX. 


\section{CALYCIFLORE. \\ XIII.-PAPILIONACEA. L。 \\ Diadelplia, Decandria, $L$. \\ 1. LOTE..}

Ono'nis L.-Rest-harrow. Bugrane, Fr. Hauhechel, Ger. rotundifólia L. p. 7. Rocky.

Scanfs. C. xlix.

Anthy'llis L.-Kidney Vetch. Wundklee, Ger:

Vulnerária L. p. 6-7. B. Dry pastures.

common. I.

Medica'go L.-Nedick. Imzerne, Fr. Schneckenklee, Ger. falcáta L. p. 7 - 8. B. Dry meadows and banks.

Samaden. L.

Melilo'tus 'Tourn.-Melilot. Honigklee, Ger.

arvénsis Wallr. b. 7 -8. B. Waste.

Samaden. LI.

Trifo'lium L.-Trefoil and Clover. Trèfle, Fr. Klee, Ger. alpínum L. p. 6-9. Pastures, etc.

common. LII.

bádium Schreb. p. 6-9. Moist meadows and pastures. Silvaplana. Lir.

Lo'tus I. - Bird's-foot Trefoil. Lotier, Fr. Schotenklee, Ger. corniculátus L. p. 6 -8. B. Pastures.

Pontresina. LIIl.

Pha'ca 1.-Bastard Vetch. Berglinse, Ger. alpina Jacq. p. 6--7. Stony. frígida L. p. 7-8. High pastures. austrális L. p. 7-8. High rough pastures. astragalína D C. p. 6-8. Pastures, etc.

Silvaplana. I.IV. Lunghino. C. LV.

Pontresina. LVI.

common. IVII.

Brail. C. L.viı.

Pontresina. Lviı.

Saluver. C. Lix.

lappónica Gaud. P. 6-7. High : peduncles Jong. Saluver. C.
montána DC. p. $6-7$. Pastures; peduncles bardly exceeding Jeaves.

Albuja. C. Lviı.

\section{VICIE.E.}

Vi'cia L. - Vetch, Tare. Vesce, Fr. Wicke, Ger. Veccie, It. sépium 1. p. 6-7. B. Shade. Crácca I. J. 6-8. B. I)ry.

Pontresina. Lx.

Pontresina. Lxi.

La'thyrus I.-Vetchling. Gesse, Fr. Platterbse, Ger. praténsis L. p. 7 -S. B. Meadows.

Pontresina. Lxil.

\section{3.- HENYSAREA.}

Hippocre'pis L.-Horseshoe Vetch. Hufeisenklee, Ger. Ferro di comósa L. p. 6--7. B. Banks.

Ono'brych is Tourn.-Saintfoin. Esparcette, $F$. Cedrangola, $I t$. montána 1) C. p. 6-7. Neadows.

Hedy'sarum L.-Sainfoin, Fr. Süssklee, Ger. obscúrum L. p. 6-7. Moist pastures.

Pontresina. J.xiv.

Meierei, Lxv. 


\section{XIV.-ROSACEA JUSS.}

I. AMYGALE.

Ce'rasus Juss.-Cherry: Cerisier, Fr. Kirschbaum, Gio: Ciriegio, It. lcosandria, ITonogyria, i.

Pảdus I) ( . I. S. 7. B. Dry. Rosegthal. ixw.

\section{ROSE.F.}

Sanguisor'ba L.--Burnet. Pimprenelle, fr. Wiesenknopf, (ier:

\section{Titrindrit, Monogluin, L.}

officinalis 1. 1. 7 - 8. B. Moist meadow's.

Stitzer-see. Lxwll.

Alchemi'lla 1..-Lady's Mantle. Sinau, Ger. Tetrindria, Mono,'sma, L. alpina 1. p. $6-8$. 13. High rocky.

Albula. C. Lxisis. vulgaris 1. p. $6-7$. B. (irassy hills.

common. Lxis11.

Dry'as 1.. - Mountain Avens. Dryade, Fr. and Ger.

$$
\text { Icosandria, Polygyniu, } L \text {. }
$$

octopétalir 1.. p. 6 -8. B. Woods, pastures.

sils. LXIX.

Ge'um 1. Arens. Benoite, Fr. Icosandria, Polygynia, $L$. rivále I. p. $6-7$. B. Neadows. Silvaplana. reptans L. p. 7-8. High rocky; terminal leaflet not much larger than the others. Cambrena. montinum 1. p. 6-8. Pastures; terminal !eaflet large. I anguard.

Lxix.

LXX.

Lxxi.

Sibba'ldia L.

Pentindria, Pentagynia, $L$.

procúmbens L. p. $7-8$. ]3. High.

liernina. 1.xxı.

Potenti'lla l._-Cinquefoil. Quintefeuilie, Fr. l̈̈ngerkraut. Ger. Cinıpuefoglio, $\mathrm{It}$.

Tiosandivia, Polysumit, $L$.

Anserina I. p. $6-8$, B. I)ry.

common. Lxir.

argéntea, 1. p. 7 -8. B. Dry.

Pontresina, Lxisis.

vérna I. p. 6 7. B. Dry. Lower stipules with a linear tail. Meierei.

LxxแII.

maculita l'ourr. var. firma, p. 7 -8. li. Dry:

bernina. Lxxıv.

aúrea L. p. 6-7. Grassy; stipules not prolonged.

l'ontresina. Lxxill.

cauléscens L. p. 8-9. Fissures of rocks.

grandiflóra I. p. 6-8. Hill sides and pastures.

Tormentilla Sibth. p. 6-7. B. Woods, etc.

Madulein. C. Lxxv.

Pontresina. cxxir.

common. Lxxill.

Co'marum L.-Narsh Cinquefoil. Comaret, Fi: Siebenfingerkraut, Ger. paluistre L. p. 7-8. 13, Wet. Jcosandriu, Polyeynia, $L$.

Fraga'ria 1.- Strawberry. Fraisier, Fr. Erdbeere, Ger:

Stiatzer-See. Lxxvir.

vésca 1. p. 6-7. B. Woods, etc. Iiosandria, Polygynia, L.

Pontresina.

LXXili

Ru'bus L.-Bramble. Ronce, Fr. Brombeere, Ger. Rovo, It. liosandria, Polyzynia, L.

Ide'us L. s.S. 7. B. (Raspberry.) Woods.

Pontresina.

Lxixim.

Ro'sa L.-Rose.

Kosandria, Monegria, $L$.

alpina L. s.S. 7 - S. Hill sides; no prickles.

Silvaplana.

1.xxin. 


\section{3.--Poмe.}

Py'rus L.-Pear.

Icosandria, Pentagynia, L.

aucupária Gertn. (Mountain Ash. Sorbier des oiseleurs, Fr. Vogelbeere, Ger.) T. 7-8. Woods.

Arnela'nchier Mled.-Felsenbirne, Ger: vulgáris Mœnch. S. 6-7. Rocky hills.

Cotonea'ster Med.-Steinapfel, Ger. vulgáris Lindl, s. S. 6 -8. B. Rocky.

Pontresina.

LXXix. Icosandria, Pentagyinia.

Pontresina. Lxxx.

Icosandria, Pentagynia, L.

Pontresina. Lxxx.

XV.--ONAGRARIEA JusS.

Epilo'bium L.-Willow-herb. Weidenröschen, Ger.

spicátum Lam. p. 7--8. B. Woods.

Octandria, ATonogynia, $L$.

Fleischéri Hochst. p. 7-8. Stony.

Silvaplana.

LXXXI.

Surlej. Lxxxi.

montanum I. P. $7--8$. B. Shady hills. Stigma four-cleft; no raised lines on the stem.

Pontresina.

palústre L. p. 7 -8. B. Wet. Stigma undivided; no raised lines on the stem.

Pontresina.

alpínum L. p. 7-8. B. Wet. Raised lines on stem; leaves obtuse; autumn shoots above ground, green.

Pontresina.

origanifólium Lam. p. 7-8. B. Wet. Raised lines on stem, leaves acuminate, autumn shoots underground, yellow. Pontresina.

LXXX11.

LXXXII.

LXXX111.

LXXXII.

\section{XVI.-CA LLITRICHINEA Lk.}

Calli'triche L.-Water Star-wort. Wasserstern, Ger. Monandria, Digynia, L.

stagnális Scop. var: verna, a. or p. 6-8. B. Styles evanescent.

Pontresina.

Lxxxiv.

\section{XVII.-TAMARISCINEA Desv.}

Myrica'ria Desv.-Tamarisk.

Decandria, Trigynia, $L$.

germánica Desv. S. 6 - 7. Gravel of rivers.

Norteratsch.

Lxxxiv.

XVIII.-PORTULACE $Æ$ Juss.

Mo'ntia L.-Blinks.

fontána L. var. rivuláris, p. $7-8$. B. Rills.

Triandria, Trigynia, $L$.

Pontresina. C. txxxiv.

\section{XIX.-PARONYCHIE A St. Hill.}

Hernia'ria L.-Rupture-wort. Bruchkraut, Ger.

$$
\text { Pentandria, Digynia, L. }
$$

glábra L. p. $7-8$. B. Try, sandy, or stony.

Samaden. Lxxxiv.

Sclera'nthus 1.-Knawel. Gnavelle, Fr. Knäuel, Ger.

ánnuus L. a. b. 7 -8. B. Dry.

Decandria, Digynia, L.

Pontresina. Lxxxiv. 


\section{CRASSULACE无 IC.}

Sedum I.-Stonecrop. Orpin, Fr.

atrátum 1. a. $6-7$. 1)ry, stony.

Decandria, Pentagynia, L.

villósum 1. a. 1) C., b. Koch, p. Sim. 8. B. lioggy.

Silvaplana. Lxxxy.

annuum 1. 1. 6 -8. Dry stony. No creeping shoots.

Rosegthal. Lxxxv.

álbum 1. p. 7 8. 13. Walls, rocks, and dry banks.

Pontresina. Lxxxy.

dasyphy'llum 1., p. 6-7. B. Wall and rocks. Leaves glaucous.

Silvaplana. Ixxxy.

acre I. p. 6-8. I). Walls and dry. Petals acuminate, leaves oroid, with a rounded protuberance at base.

Pontresina. 1.xxxir.

répens Schleich. p. 6-8. High rorks. P’etals obtuse, leaves sessile, not produced at base.

l.anguard.

Sempervi'vum I.. Houseleek. Joubarde, Fr. Hauswurz, G'r. Semprevivo, Il.

tectórum L. 1). 7 S. B. Rexks.

Dodecandria, Dodecagynia, $L$.

arachnoideum L. p. 7 -8. Rocks.

Silvaplana.

common.

Wulféni Hpe. p. 7 -8. High rocks.

Lake Cavloccio.

\section{XX1. GROSSULARIEA D C}

Ri'bes I._-Currant. Groseiller, Fr. Johannisbeere, Ger.

* rúbrum 1. S. 6-7. I3. Woods.

Pentandria, Monogrnia, $L$.

alpinum 1. s.S. 6 - 7. B. Woods.

Pontresina. Ixxxix.

St. Moritz. Lxxxix.

\section{XXII.-SAXIFRAGEA Juss.}

Saxi'fraga 1.-Saxifrage. Steinbrech, Ger. Decandria, Digynia, L.

Aizóon !aç. p. 6-8, Rocks.

crésia 1. p. 7 - 8. High.

oppositifólia I. p. 5-S. Is. High rocks.

stelláris L. p. 6-S, B. Wet.

áspera I. P. 6-8. Stony.

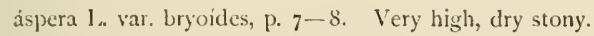

aizoides L. P. $7-8$. B. Moist.

cuneifólia 1. p. 6-8. Shady rocks.

planifólia Lap. p. 7-8. High rocks.

exarata Vill. p. 6 -8. Dry.

androsicea I. p. 6-8. Moist, stony.

Seguiéri Spr. p. 6 -8. Very high.

rotundifólia L. p. $7--8$. Moist shady.
Silvaplana. $\mathrm{xc}$.

Piz .llv. C. xc.

Surlej. xc.

Silvaplana. $\mathrm{xc}$

Sils. $\mathrm{xer}$.

Bernina, xcl.

Silvaplana. xсr.

Silvaplana. xerr.

Surlej. xell1.

Silvaplana. xсн1.

Mlorteratsch. xcill.

Beversthal. C. хси.

Silvaplana. $\mathrm{xer}$.

- This is probably Rile's petrexum Wulf, an undoulted Jingadine plant. R. rubrum, except as a garden escape, would not be founcl at su high an elevation. 
Chrysosple'nium L. -Golden-Saxifrage. Dorine, Fr: Milzkraut, Ger: Decandria, Digymia, L.

alternifólium L. p. 6. B. Moist shade.

St. Moritz. xcv.

XXIII.-UMBELLIFER E Juss.

Pentandria, Digynia, $L$.

Astra'ntia L.-Astrance, Fr.

mínor L. p. $7-8$. High. Rosegthal. xcri.

Bupleu'rum L.-Hare's-ear. Buplère, Fr. Hasenohr, Ger.

stellátum L. p. 7-8. Hill sides. Pontresina. xcri.

Me'um Jacq. - Spignel. Bärenwurz, Ger.

Mlutellina Gertn. p. 6 - 8 . Pastures. P’ontresina. xcrii.

Laserpi'tium L.-Laser, Fr. Laserkratt, Ger.

hirsútum Lam. 7 -8. Meadows. Samaden. xcyiri.

XXIV. CAPRIFOLIACEA Rich.

Ado'xa I.-Moschatel. Moscatelline, Fr. Bisamkraut, Ger.

Octundria, Tetragyini, L.

Moschatellina L. p. 6. Shade.

Albula. C. xcr:

Sambu'cus L-Elder. Sureau, Fr. Hollunder. Ger.

Pentandria, Trigyia, $L$.

E'bulus L. p. $7-S$. B. Uncult. Herbaceous. Pontresina. C.

Pontresina.

xeIx.

racemósa I. 1.S. . S. Stony woods.

c.

Lonice'ra L.-Honeysuckle. Chèrefeuille, Fr. Geisblatt, Ger. Madreselva, It.

Pentandria, Monogynia, L.

nígra L. S. 7. Woods. Berries laterally adhering. Crestalta.

alpígena L. s. S. 7. Woods. Berries united into one.

Crestalta.

cærúlea 1. S. 6-7. Woods and open.

Pontresina.

CI.

Cr.

Linnæ'a (iron.

Didyamia, Anginspermia, $L$.

boreális Gron. p. 7 -9. B. Mossy pine woods.

St. Moritz.

C11.

\section{XXV.-RUBIACE压 Juss.}

Ga'lium L.-Bedstraw. Gaillet, $F$ : Labkraut, Ger. Gaglio, It. Tetrandria, Monogynia, $L$.

boreále l. p. 7 - . B. Heathy. Leaves in fours, three nerved.

vérum L. p. 7 - S. B. Dry banks.

Silvaplana. CIII.

Samaden. Cril.

sylvéstre Poll. p. 6-7. B. Rough, rocky. Leaves $6-8$, one nerved.

Silvaplana. CIIı.

uliginósum L. p. 7. B. Wet. Stem rough with reversed prickles.

Samaden. CIII. 
XXVI,- VALERIANE王 I)C.

Valeria'na 1.-Valerian. Baldrian, (i'r. Triandrit, I/onosynia, /..

officinális L. p. 7 8. B. Moist banks and woods. Pontresina. (cw.

montana L. .) $7_{-S}$. Shady rocks. Surlej. $\mathrm{Cl}$.

tripteris I. P. 5-8. Rorky. Sometimes direcious. P’ontresina. cri.

\section{XXVII.-DIPSACEA DC:}

Knau'tia Coult. - Scabious.

Tetrandria, Monogymia, $L$.

arvénsis Coult. p. 7 -S. I3. Fields.

Scabio'sa l..--Scabious.

common. ivil.

Columbaria [. var. lícida, p. 6 -8. (irassy hillsides. Stät/er-See. 1vm.

\section{COMPOSIT A Idans. Syngenesia $L$. \\ 1. Corsunirkr.}

\section{Homogy'ne Cass.}

alpina Cass. p. $6-7$. Woods and pastures.

common. cIx.

Solida'go L. Ciolden-rod. V'erge d'or, Fr. Goldruthe, Ger. Verga d'oro, $I t$.

Virga-aúrea I. 1. 7 - - S. 13.

common. $\mathrm{cx}$.

A'ster 1.-Star-wort. Sternblume, $G(r$.

alpunus 1. 1). 7-9. Rucky pastures, etc. Pontresina. cxı.

Bellidia'strum Cass. l'âtpueronic, $F r$ :

Michélii Cass. p. 6-7. Morders of woods. St. Mority. cxi.

Be'llis I. Daisy. Pâquerette, fir: Maasliebe, Gir. Margheritina, It. perénnis L. p. 5 -9. [3. Meaduws, etc. common. cx1.

Eri'geron J.-. I'lea-bane. Vergertte, Fr. lierufkraut, Ger.

ácris L. var. drcebachénsis, p. $7-8$. Dry. Leaves hairy only on the margin.

Morteratsch. cxil.

Villarsii 13ell. $1 \% 7^{-8}$. Hill sides.

Pontresina. cxil.

alpínus I. p. 6-8. I3. l'astures.

common. cxiv.

uniflórus I. P. 6-8. High grassy:

languard. cxiv.

Gnapha'lium I..-Cudweed. Ruhrkraut, Gir.

sylvaticum L. P. $7^{--8 . \text { B. Woods. }}$

supinum I. p. 7 ..S. ls. Very high.

Icontopódium Scop) p. $7-8$. Very high.

dioicum I. p. $6-8$. 1). I)ry.

carpáthicum Wahl. p. 7-S. High moist rocks.

Surlej. cxv.

Bernina. cxv.

Julier. cxv.

common. CXv.

fexthal. C. cxi'.

Artemi'sia 1.-Wormwood, Mugwort. Armoisc, li: Wermuth, Ger.

spicata Wulf. P. 7 8. High rocks.

Fout of l'iz Corvatsch. C. cxv.

Mutellina Vill. p. $6-8$. High open rocks.

Rosegthal. cxit. 
Leuca nthemum Lam. - Ox-eye.

vulgáre Lam. p. $6-8$. B. Meadows, etc.

common. cxvil.

* coronopifólium Vill. p. 6-7. High.

Saluver. C. cxvil. alpínum Lam. p. 6-8. High stony.

Morteratsch. cxvit.

Achille'a L.-Yarrow, Sneezewort. Schaafgarbe, $G c \%$. nána L. p. 7-8. Very high.

Rosegthal. cxint. moscháta Jacq. p. 6-8. High.

Rosegthal. cxvitr. hy'brida Gaud. p. 7 -8. Very high. Hybrid between moschata and nana.

atráta I. p. 7 -S. High.

Millefólium L. p. $7 \rightarrow 9$. B. Pastures, etc.

Bernina. C. cxix.

common. cxix.

A'rnica L.-Wohlverleih, Ger.

montána L. P. 7 -8. Open woods, etc.

common. cxx.

Aro'nicum Necker.

Clúsii Koch. p. 6-8. High stony. Stem hollow.

Cambrena. cxxi.

Sene'cio L.-Groundsel or Ragwort. Seneçon, Fr. Kreuzkraut, Grr:

viscósus $L$. a. 7 -8. B. Stony woods and waste.

Pontresina. cxxi.

abrotanifólius L. p. 7-9. High.

incánus I. var. carniólicus, p. 7-S. Very high.

Silvaplana.

cXxil.

nebrodénsis L. a. or. p. 7- $\delta$. Rocky pastures.

Bernina. cxxi.

Fúchsii Gmel. p. 6-8. Woody hills.

Silvaplana. cxxit.

Dorónicum L. p. 6-8. High rough pastures.

Rosegthal. cxxrr.

Pontresina. cxxy.

\section{Cynarocephat.Æ.}

Ci'rsium Scop.-Plume-Thistle. Kratzdistel, Gir.

erióphorum Scop. p. $7-8$. B. Dry.

Samaden. cxxvi.

Carli'na L. Carline-Thistle. Eberwurz, Ger. acaúlis L. p. 7 -8. MLountain pastures.

Silvaplana. cxxvit.

Saussu'rea DC.

alpína DC. p. 7-8. B. High meadows.

St. Moritz. Cxxvill.

Serra'tula I.-Sawwort. Sarrête, F\%: Schartendistel, Ger. Rhapónticum DC. p. $7-8$. Rough meadows.

Pontresina. cxxix.

\section{3.- CHICORACE.}

Hypochæ'ris 1.-Cat's-ear. Porcelle, Fr. Ferkelkraut, Ger. uniflóra Vill. p. 7-8. Rough pastures.

Silvaplana. cxxx.

Leo'ntodon 1.-- Hawkbit. Liondent, Fr. Löwenzahn, Ger.

híspidus L. p. 6-9. Meadows and pastures.

common. cxxxr.

* According to Nyman (Conspectus Flore Europrex) this plant should be named I. ceratophylloides Nym. It is the same as Pyrethrum ! Malleri WW., and is distinct from Chrysanthemum (not Lcucanthenum) coronopifolium of Villars, which is not given for Switzerland at all. 
Tragopo'gon 1. (ioat's-beard. Salsifis, fir. Bocksbart, Ger. Barba di beceo, $/$.

praténsis I. b. 6 7. B. Neadow's, ctc.

Pontresina. cxxxi.

Tara'xacum Hall.- Mandelion. I'faffenrö̈rlein, Ger. officinale Web. var. palústre, 1) 6-7. B. Marshes. Stätzer-Sce. (xxxut.

Cre'pis 1.- Hawk's-beard. l'ippau, G'\%. aúrea Cass. p. 7 - 8. P’astures, etc. Jacepuini Tausch. p. 7 - 8 . Stony. hyoseridifólia 'lausch. p. 7--8. Vicry high.

Statzet-sce. cxxiti.

Silvaplana. cxxxu.

Albula. C. cxisin.

Alp Murail. C.. cxxxur.

Hiera'cium I. Ifawkweed. Epervière, fr: Habichtskraut, Gir:

Pilosélla L. p. 6 -9. B. Lry. liracts alt lanceolate acute. Silvaplana. cxxxiv.

pillosellitúrme I)C. p. 8. 3ry. Outer bracts ovate. Saluver. C. cxxxiv.

aurantiacum 1. p) or b. 7 - . High pastures. St. Moritz. cxxxr.

Auricula L. 7 9. lilowers $2-5$ crowded into a head. Pontresina. cxxxr.

alpinum 1. p. 7 -8. B. High. Hernina. cxxxvi.

glanduliferum Hopp. 1. 7-8. Very high. Bernina. cxxxr.

villósum L. var. dentatum, p. 7 . Open rocks. Surlej. cxxxvit.

scorzonerifólium Vill. p. $7-8$. Stony. Rosegthal. cxxxwri.

albidum Vill, p. 7 -8. Rocks and gravel. Very viscid. Samaden. cxxix.

XXIX.-CAMPANULACE $A$ Juss.

Campa'nula 1..--Bell-flower. Glockenblume, Ger. Campanella, It.

Pintendia, IVonogyinit, $L$.

thyrsoidea 1. p. $6-7$. Pastures.

Sils. cxi.

barbata L. p. 7 8. Woods and hill sides.

St. Moritz. cxil.

rapunculoides L. p. 8. B. Stony.

Samaden. cxis.

pusilla Hank. p. $7-8$. Rocks and gravel of torrents. Flatz-bach. cxitu.

Ścheuchzéri Vill. p. $6-8$. Pastures, etc.

Pontresina. cxirti.

Phyteu'ma L. Rampion. Raiponce, Fr: Rapunzel, Ger: Raperonzolo, $/ 1 . \quad$ Pentandria, Monogynia, $L$.

paucifórum 1. p. $7-8$. Very high. Heads $5-7$, leaves obovate.

Languard. C. cXlil:

hemisphe'ricum L. p. :-8. High. Heads ro-15, leaves linear.

humile Schieich. P. 6-7. Very high rocks. Rusegthal. cxus:

orbiculaire L. p. 6 - 8 . B. Pastures. common. cxis.

Scheuchzéri All. p. 7 S. Rocks. Silvaplana. cxlr.

betonicifóliun Vill. p. 6-S, Hill meadows. St. Moritz. cxlv'

- Halléri $A$ Nl. p. 7 -8. Woods and pastures. Silvaplana. exirul.

- This is named by mistake on the plate Plyteunit spicatum var. nigrum. 


\section{XXX.-ERICE E Juss.}

Arctosta'phylos Adans.-Bear-berry. Bärentraube, Ger:

Decandria, Monogynia, L.

officinális Wimm. s.S. $6-7$. B. Woods.

common. CXIVIH.

Callu'na Salisb.--Ling.

Oitandria, Monogynia, $L$. vulgáris Salisb. s. S. 7. B. Heathy.

Stätzer-See. cxlvil.

Eri'ca L.-Heath. Bruyère, Fr. Heide, Ger. Octundria, Monogynia, L. cárnea L. s.S. 5 -6. Woods.

Morteratsch. cxLviII.

Aza'lea L.

procúmbens L. s. S. 6-7. B. Rocky.

II uottas. cxus.

Rhodode'ndron L.- Rosage, Fr. Alpenrose, Ger.

Decandria, Monosynia, $L$.

ferrugineum L. s.S. 6 -9. Rocks and pastures.

common. cxLIX.

hirsútum I. s.S. 7-8. Rocks.

Celerina. C. cxtix.

Vacci'nium L.- Whortleberry. Airelle, Fr. Heidelbeere, Ger.

Octandria, Llonogy'nia, L.

Myrtillus L. w. 6. B. Woods.

common.

uliginósum L. s. S. 7. B. Boggy.

Pontresina.

Vítis-ldæ'a L. w. 6-7. B. Woods.

common. CLL

Oxyco'ccus Tourn.-Cranberry. Canneberge, Fr.

Oitandria, Monogryin, L.

palústris Pers. w. 7. B. Bogs.

Stätzer-See.

ClI.

Py'rola L.-Wintergreen. Wintergrün, Gcr. Decandria, Monggynia, L.

rotundifólia L. p. 7 -8. B. Moist woods.

Below Crestalta.

Clil.

minor L. p. 7 -8. B. Woods.

common. Clilt.

secúnda L. p. 7-8. B. Shady woods.

St. Moritz. clil

uniflóra L. p. 7-8. B. Shady woods.

St. Moritz. CLII

\section{COROLLIFLORE.}

XXXI.--GENTIANEA Juss.

Gentia'na L.-Gentian. Enzian, Gcr. Genziano, It.

Pentandria, Disynia, L.

lútea L. p. $7-8$. Woods.

Rosegthal. CLIN.

Charpentiéri Thom. p. 7--8. Woods. Hybrid between punctata and lutea.

Rosegthal. Cr.

punctàta I. p. $6-8$. Woods, etc.

common. CLVI.

cruciáta I. p. 7-9. Iry pastures.

Samaden. CLV11.

asclepiadéa 1. p. 8 9. Grassy thickets.

Pontresina. C. CLrill.

ciliáta 1. p. $7^{-}$9. Rough pastures.

Sils. CL1X.

excísa Presl. p. 6-8, Mleadows and woods. Teeth of calyx spreading. 
bavarica I. P. 7 - S. High moist. Style deeply cloven. common. cLix.

brachyphy'lla Vill. p. $7^{--8}$. Very high. Style undivided; leaves sulyrotundo ovate.

Bernina. (I.).

virna 1. p. 6 7. 13. Meadows, etc. Style undivided; leaves elliptic, acute.

common. cIX.

utriculósa L. a. 7 -S. Moist. Calyx inflated with winged angles.

nivális $\mathrm{I}$.. a. $6-\mathrm{S}$. I. Meadows, etc. common.

Silvaplana. $\mathrm{CLx}$.

yermanica llilld a 8 - 9. I3. Meadows, etc. Leaves acute. Fonte, C.

obtusifólia Wilkt. a. 7 -8. Iry. Lower leaves obtuse. Maloja. (ז.xi.

campéstris I. a. 6-S. 1). Open. Often white. common. cLxi.

tenella Kottb. a. 7-8. Edges of torrents. Morteratsch. cr.xı.

XXXII,- POLEMONIACEA Ven:

Polemo'nium L.-Jacob's-ladder. S̈perrkraut, Ger.

Pintandria, Monogrinia, L.

carúleum I. P. 7-8. B. Moist woods.

Crestalta. crxu.

XXXIII.-CONVOLVULACE

Cu'scuta L.--I)odder. Flachsseide, (ror. Pentendria, Digynia, L. europexa L. a. 7 -8. B. l'arasitic on herbs, etc. Silvaplana.

CLSII.

\section{BORRAGINEA Juss.}

E'chium L.-Viper's-Bugloss. Vipérine, Fr: Naterkopf, Ger.

Pentandria, Monogyula, L. vulgare I. b. $6-8$. I3. Dry uncult.

Meierei. ст.хи.

Pulmona'ria 1.-1.ungwort. Lungenkraut, Ger:

Pentandria, Jonosynia, $L$.

azírea Bess. p. 5-6. B. Stony shade.

Morteratsch. CLxill.

Myoso'tis I.-Mouse-ear. Näuseohr, Ger. Orecchio di topo, It.

Pentundriu, Monogyiu, L.

alpéstris Schmidt. p. 6-8. B. Open.

common. crixs.

Eritri'chium Schrad.

Pentandril, Monogynia, $L$.

nánum schrad. p. 7 - 8. Very high granitic.

Bernina. cLxiv.

\section{XXXV.--SCROPHULARINEA JusS.}

Verba'scum I.--Nullein. Ioléne, Fr. Wollkraut, Ge\%. Tasso barbasso, It.

Pentandria, Mlonogrinit, L.

? pulverulentum Vill. b. 7--\$. B. Stony hills. Pontresina.

CI.XV.

Lina'ria Juss. - Toadflax or Snapdragon. Leinkraut, $\mathrm{Gcr}$.

Didjnamia, Angiospermia, L.

alpína Mill. a - b. 7 -8. Stony and gravel of torrents. Silvaplana.

vulgáris Mill. P. 7 - 9. B. Uncult.

Celerina. C. cixv. 
Euphra'sia I.- Eyebright. Augentrost, Ger.

Didynamia, Angiospermia, $L$.

officinális 1. var. alpína, a. 6-8. Pastures, etc.

Morteratsch.

CLXVII. minima DC. a. $7-8$. Nountain meadows.

Silvaplana. CLxvı.

Ba'rtsia L.

Didinamia, Angiospermier, $L$.

alpina I. [. 7 -8. B. Moist.

common. CLXVII.

Melampy'rum L.-Cow-wheat. Wachtelweizen, Ger.

Didynamia, Angiospermia, $L$.

praténse L. a. 6-8. B. Woods.

Silvaplana.

CLXVill.

sylváticum I. a. $6-8$. B. Woods.

Silvaplana.

CLXVIII.

Rhina'nthus L. Yellow-rattle. Cocréte des prés, Fr. Klappertopf, Gir. Cresta di gallo, $I t$.

Didynamia, Angiospermia, $L$.

májor Ehrh. a. 6-7. B. Meadows. Tube curved, style protruding.

Pontresina.

CLXIX.

minor Ehrh. a. 6 - 7. B. Moist neadows. Tube straight, style included.

CIXIX.

Pedicula'ris Tourn. - Lousewort or Red-rattle. Läusekraut, Ger. Pidocchiera, $I t$.

Didinamia, Angiospermia, $L$.

rostráta 1 .. p. 6--8. High moist.

languard. CLXX.

incarnáta Jacq. p. 7 -8. High novist.

Beversthal. C.

CLXXI.

tuberósa L. p. 6-8. Grassy.

Samaden.

CLXXI.

átro-rúbens Schleich. p. 7--8. High grassy.

Beversthal. C.

palústris 1. p. or b. 6-7. B. Boggy meadows.

Stätzer-See.

cI.xill.

foliósa L. p. $7-8$. Stony.

Rosegthal.

CLXXII.

recutita L. p. 6-8. Moist meadows.

Pontresina.

CLXXIII.

verticilláta L. p. 6-8. Woods, meadows.

Pontresina.

CIXXIV.

CLXXV.

Vero'nica I..-Speedwell. Ehrenpreis, Ger. Diandria, Lonogymia, $L$.

Beccabúnga L. p. 6-8. B. Wet.

Pontresina.

cixixv.

Chamæ'dry's 1. p. 6-7. B. 1)ry.

Pontresina.

CLXXVI.

officinális L. p. 7-8. B. Woody hills.

Crestalta.

CLXXII].

spicáta L. p. $7-8$. B. Dry.

bellidioides L. p. 6 - 8 . Grassy.

Pontresina. C.

CגxXVI1.

saxátilis Scop. w. 6-7. Rough stony.

Pontresina.

cLxxVII.

alpina L. p. 6-8. B. Grassy mountains.

Silvaplana.

CLXXVIII.

serpyllifólia L. p. $6-7$. B. Meadows, etc.

Stätzer-See.

CLxxvil.

Pontresina. CLxxiul.

\section{XXXVI.—LABIAT $Æ$ Juss.}

Didynamia, Gymnospermiar, L. Except Salvia.

Sa'lvia 1.--Sage or Clary. Sauge, Fr. Salbei, Ger:

Diandria, Monogynia, L.

jraténsis L. p. 7--8. B. Grassy hills.

l'onte. (. CLxix.

Thy'mus L.-Thyme. Thymian, Ger:

Serpy'llum L. w. 6--8. B. Open dry.

common. CLXXX. 
Calami'ntha Meench,-Calamint. Calament, Fr. liergmünze, Gor.
alpina lam. p. 6 -S. Hill sides.
Silvaplana. (זxxx.

Hormi'num I. Jrachenmal, Cier

pyrenaicum $\mathrm{I}$. 1). 7 . High pastures.

liernina. (: cLxx.

Dracoce phalum L.--1)ragon's-head. Irachenkopf, (ier.

austriacum 1.1 \%. 7 . Stony slopes.

Ruyschiana I. p. 6. Hill sides.

Aruez. C. ILxxxi

pontresina. crxxis.

La'mium I.. - Dead-nettic. I amier, Fr. Taubnessel, Ger: Ortica monta, I/. ilbum I. 1), G-9. I). L'ncult.

common. c1.xxxנs.

Prune'jla 1. - Self-heal. Brunelle, Fr: and Ger.

vulgaris 1. p. $7-8$. 13. Woods. 1)ry pastures. common.

alpina 'limb.-1 agr. 1) 7-8. Dry calc, hills. Cor. more than twice as long as caly:

Maloja. (:. ci.xхxı!.

A'juga 1.-Bugle. (iünsel, G'r'r. Bugola, $/ \ell$.

pyramidalis L. J. G-7. B. Woods and pastures. common.

cixxill.

Teu'crium L. Germander. (iemandree, fir Gamander, Gir. Camedrio, $I t$.

montinum I. w. 7 - 8 . Calc. hills.

sils.

CLXXX111.

\section{XXXVII, LENTIBULARIEA Rich.}

Pingui'cula Tourn.-Butterwort. Grassette, fir: Fettkraut, Gir. Diandria, Monogyria, L.

vulgáris 1. p. 6 8. B. liogs. Corolla veinless. Silvaplana. grandifóra lam. p. 6 - S. 13. Bogs. Margin of corolla veined. Fex. alpina 1. p. 6-7. B. High. Silvaplana.

CLXXXI:

CLXXXIS,

CLXXXI:

\section{XXXVIII.-PRIMULACEÆ Vent.}

Prímula L.- Primrose and Cowslip. Primeviere, Fr: Primel, Ger: Primavera, $l l$.

Pentandrit, . Monogynit, $L$.

officinális Jacq. p. 6. B. Meadows.

clitior Jacq. J) 5 - 6. B. Woods.

farinósa L. p. 6-8. B. Wet meadows, etc.

Sils.

CLxxxy.

Jongiflóra All. p. 6. Very high pastures.

common.

cLxixvi.

gráreolens Heg. p. 6-7. High rocks.

Fexthal. C.

Clxxxill.

Iluottas.

CLxxxvil.

viscósa Vill. p. $6-7$. High rocks.

common.

CLxxxix.

integrifúlia L. 1). 6-7. Iligh boggy:

Muottas.

cxc.

cxic.

dinyana lagg. p. 6-7. High. Probably hybrid between integrifolia and viscosa (Nyman), or integrifolia and graveolens, according to other authors.

Albula. C. cxc.

glutinósa Wiulf. p. $6=8$. High.

Scarlthal. C.

cic. 
Andro'sace L.-Mannsschild, Gir.

Pentandria, Monogynia, L.

glaciális Hopp. p. 6- 8 . High rocks.

Bernina.

CXCl.

Chamajásme Host. p. 6-S. High grass.

Julier. $\quad \mathrm{cxcl}$.

obtusifólia All. p. 6-8. Grass.

common. $\mathrm{cxcl}$.

Soldane'lla L.-Drottelblume, Ger. Pentandria, Monoyrnia, L.

alpina I. p. 6-7. Near snow. Scales of mouth equalling filaments.

Muottas. cxcir.

pusilla Baumg. p. 6-7. Near snow. Scales of mouth O. Muottas.

CXCII.

\section{XXXIX.-GLOBULARIEÆ DC.}

Globula'ria L.-Kugelblume, Ger: cordifólia 1. w. 7. Stony.

Tetrandriu, Monogynia, L.

Mut Maria, C. cxciII.

\section{XL.-PLANTAGINEÆ Vent.}

Planta'go L.--Plantain. Wegetritt, Ger. Piantaggine, It.

Tetrandria, Monogynia, $L$.

alpina L. w. or p. 6-7. Mountain pastures.

Pontresina.

CXCIII.

MONOCHLAMYDE E.

XL1._POLYGONEÆ JusS.

Poly'gonum L.-Persicaria. Renonée, Fr. Knöterich, Ger. Persicaria, It. Octandria, Trigynia, $L$.

Bistórta L. p. 6-7. B. Moist meadows.

Silvaplana.

vivíparum L. p. 6 -8. B. Mountain pastures.

Silvaplana.

CXciv. amphibium L. p. 6-S. B. Wet.

CXCv.

XLII.-SANTALACE无 R. Br.

The'sium l„-Bastard Toadflax. Thésion, Fr. Leinblatt, Ger.

Pentandria, Monogynia, $L$.

alpinum L. p. $6-7$. Heaths and pastures.

praténse Ehrh. p. 6-7. Hill meadows.
Celerina. cxcvi.

Celerina. cxcvi.

XLIII. THYMELEA JuSS.

Da'phne L.-Mezereon. Kellerhals, Ger. Laureola, $I t$.

Octandria, Monogynia, $L$. striảta Tratt. s.S. 6-8. Rocky.

Languard. cxcvil. 


\section{XLIV.-EMPETREÆ Nutt.}

E'mpetrum 1.-Crowberry. Camarine, fir. Rauschbeere, Go:

Dircia, Trizndria, $l$.

nigrum L. s.S. 6. I). Mountain heaths. Pontresina. cxcvit.

XLV,-EUPHORBIACEA A. Juss.

Eupho'rbia $\mathrm{I}_{\star}$ - Spurge. Wolfsmilch, Ger: Monecia, Monandria, L.

Cyparissias L. p. 6- 7 . Barren, etc. Pontresina. cxcrill.

XLVI.--SALICINEÆ Rich.

Sa'lix L.-Willow. Saule, fr. Weide, Gir. Salcio, It.

Diacia, Diandritr, L.

reticuláta L. w. $6-7$. B. Moist rocky.

Fexthal. cxcvun.

\section{MONOCOTYLEDONES.}

XLVII.-IRIDEÆ JusS.

Cro'cus Tourn.--Safran, Fr: and Gir. Zafferano, It.

Triandrit, . Konogynit, $L$.

vérnus . 1ll. p. 5-6. B. Meadows.

l'ontresina. cxcrutt.

XLVIII. ALISMACEÆ R. Br.

Triglo'chin L-Arrow-grass. 'Troscart, Fr. I)reizack, Gir: Itixandrit, .Lonogynia, $I$..

palústre L. p. 6-7. B. lioggy:

Roseythal. cxic. 


\section{XLIX.-ASPARAGINEA A. Rich.}

Polygona'tum Tourn.--Solomon's Scal. Weisswurz, Ger. (inocchietto, It. Hexandria, Monogynia, L.

vulgáre Desf. p. 6 -7. B. Woods. Pontresina.

cxcix.

Convalla'ria 1..-Lily of the Valley. Muguet, Fr. Maililie, Ger: Mughetto, It.

Hexandria, Monogruia, $L$. majális L. p. $6-7$. B. Shade.

Samaden. C. cc.

Maia'nthemum Web. ap. Wigg.-Schattenblume, Ger.

Tetrandria, Monogyia, L.

bifólium DC. p. 6-7. B. Woods.

Pontresina.

Pa'ris L.-Herb Paris. Parisette, Fr. Einbeere, Ger. Uva di volpe, $/ t$. Octandria, Tetragymu, $L$.

quadrifólia L. p. 6-7. B. Woods.

Meierei. ccil.

\section{L.一ORCHIDEÆ Juss.}

O'rchis L.-Ragwurz, Ger:

Gynandria, Monandria, L.

máscula I. p. 6. B. Woods and meadows.

Silvaplana.

cCIII.

globósa I. p. $6-7$. Mountain pastures.

Val Suvretta.

latifólia L. p. 6-7. B. Moist. Stem hollow.

Silvaplana.

maculáta L. p. 7. B. WVoods and meadows. Stem solid.

Below Crestalta. ccv.

Gymnade'nia R. Br. Gynandria, Monandria, $L$.

conópsea R. Br. p. 6-7. B. Meadows.

Silvaplana. CCVI.

odoratíssima Rich. p. 7-8. Woods and meadows.

Sils. CCVI.

álbida Rich. p. 6-7. B. Hills.

St. Moritz. Ccv11.

Habena'ria R. Br. Glnandria, Monandria, $L$. viridis R. Br. p. 6-7. B. Pastures, etc.

Silvaplana. ccvis.

Nigrite'lla Rich.-Schwarzständel, Ger:

Gynandrit, Monandria, $L$. angustifólia Rich. p. $6-8$. Hills, etc.

Silvaplana. suaveolens K. p. 7--8. Hills. Hybrid between N. angustifolia and Gymnadenia conopsea.

Silvaplana.

Corallorhi'za Hall.- Coralroot. Coralline, Fr: Corallenwurz, Ger.

Gynandria, Monandria, $L$.

innáta R. Br. p. 7. Woods.

Sils. ccrir.

Cypripe'dium L.-Lady's Slipper. Sabot de la Vierge, $F r$. Frauenschuh, Ger: Pantoffola, It.

Cálceolus L. p. 6-7. B. Woods.

Gynandria, Diandria, $L$.

Scanfs. C. ccix.

\section{LILIACEA DC.}

Li'lium L.-Lily. Lis, Fr. Lilie, Ger. Giglio, It.

Hexandria, Monogyniu, L.

Mártagon L. p. 7. Mountain woods.

Silvaplana. ccx.

bulbiferum L. p. $6-7$. Moist woody hills.

Pontresina. C. ccxl. 
Lloy'dia Salisb.-Spiderwort. Hexandria, Monorynia, $L$.

serótina Reich. 1) 6 -7. B. Stony shade.

Bernina Falls. ccxu.

Ga'gea salisb. - Vugelnileh, Gir: ITexandria, Monogryia, I.

l,jottardi Schult\%. p. 5-6. Nountain pastures, woods, etc.

Morteratsch. cCXil,

A'llium L.-Garlick. Ail, Fr. Lauch, Gir: Aglio, It.

Hexandria, Monogynia, L.

Victoriális L. p. 7-8. Rocky:

Bernina. C. ccxull.

Schrenóprasum 1. 1). 6-7. L). Sides of rivers and lakes. St. Moritz. ccxir.

Lilia'strum Tourn,-St. Bruno's lily: Faux-lis, Fr.

Ifxandria, Afonogynia, $L$.

álbum link. p. 7. Pastures.

Maloja. ccxis:

\section{COLCHICACEE DC.}

Co'Ichicum L.-Meadow Saffron. Zeitlose, $G^{\prime}{ }^{\prime}$ :

Hexandria, Trigrnia, L.

autumnále 1.. p. $\&$ 9. B. Moist meadlows.

Celerina. ccxi.

Tofie'ldia lHuds. - Scottish Asphodel.

Hexandria, Trigynia, $L$.

palústris lluds. p. 6-8. B. Boggy pastures.

Pontresina. ccxil.

calyculàta Wahl. p. 7 - 8. Boggy pastures.

Julier. ccxis.

Vera'trum L.-Niesswurz, Ger. Elleboro bianco, It.

Hexandra, Trigynia, $L$.

álbum 1. p. $7-8$. Meadows.

Silvaplana. ccxir.

\section{LIII.-JUNCEA DC.}

Ju'ncus L.-Kush. Jone, frr: Binse, Ger. (Yiunco, $1 t$.

Hexandria, Aonogyuia, $L$.

filifórmis 1. p. 7-8. B. Peaty meadows.

Morteratsch. C. ccxiru.

alpinus Vill. p. 7 - S. B. Moist.

Morteratsch. C. cexiut.

Jacquini L. p. 7. High moist.

Heuthal. C. ccxix.

triglúmis L. p. 7. B. Moist.

Plattas. C. ccxix.

trifidus 1. 1). 7-8. 1. Fissures of high moist rocks. Pontresina. C. ccxix.

Lu'zula I)(..-Wood-rush.

Hexandria, Monogynia, $L$.

lítea 1)C. p. 6-7. Pastures, woods, etc.

Pontresina. ccxi.

nivea [ C. p. 7 -S. Neadows, etc.

Pontresina. C. ccxi.

\section{CYPERACEA DC.}

Erio'phorum 1.-Cotton-grass. Linaigrette, Fr: Wollgras, Ger.

Triandria, Honogynia, $L$.

alpínum L. p. 7. B. l Bogg:

Stätzer-Sec. ccxis.

Scheuchzéri Hopp. p. 6-7. Mloist.

Bernina. ccxxi.

angustifólium Koth. p. 6-7. Boggy:

Rosegthal. ccxxi. 
Carex L.-Sedge. Laiche, Fr. Segge, Gir. Caretta, It. Monacia, Triandria, L.

echináta Murr. p. 6-7. B. Boggy. canéscens L. p. 6-7. B. Boggy.

* leporína L. p. 7. B. Moist. nígra All. p. 7-8. Dry grassy. limósa L. p. 6-7. B. Bogs. panicea, L. p. 7. B. Springy meadows. pallesscens L. p. 7. B. Moist. fláva L. p. 6-7. B. Boggy. vesicária $L$. p. 7. B. Water.
Stätzer-See. C. ccxxı.

Stätzer-See. C. ccxxu.

cсxxi1.

Piz Languard. C. ccxxilı.

Morteratsch. C. ccxxilı.

Stätzer-See. C. ccxxm1.

Stätzer-See. C. cсххм.

Stätzer-See, C. ccxxiv.

Stätzer-See. ссххж:

* This is probably C. Jagopina Wahl., whicb, though very near C. leporina, is a smaller and essentially alpine plant, growing according to Her Caviezel in Val Bevers. I have not preserved the specimen from which the drawing was made, nor have $I$ any note of where I gathered it. 

I N D E X. 



\section{INDEX OI: THE LATIN NAMES.}

\section{Sinonyms are in Malics.}

\begin{tabular}{|c|c|c|c|c|}
\hline ACHIIII:I & & & l'age. & Plate \\
\hline itrati & 78 & $\ldots$ & 12 & cxix. \\
\hline hybricla & $\ldots$ & $\ldots$ & 12 & (xilli. \\
\hline Millefolium & $\ldots$ & $\ldots$ & 12 & (XIX. \\
\hline mosthiatia & $\ldots$ & $\ldots$ & 12 & (xilll. \\
\hline mana... & $\ldots$ & $\ldots$ & 12 & cxills. \\
\hline
\end{tabular}

Achyophorms /lelveticus. Hypochicris uniflora. Acosilis

Cemmarzm, paniculattm.

Lycoctonum $\quad \ldots \quad 2$ xis.

Nipellus $\quad \ldots \quad \ldots \quad 2$ xim.

panteulatum $\quad$... 2 xitl.

aturiegatum. paniculatum.

ADONi.

Mosclittellina $\quad \ldots \quad 10 \quad \mathrm{xCl}$.

A.srostemmer. Lychnis.

Ajti:it

pyramidalis ... $\quad \ldots \quad 17 \quad$ c1.s.x.

Al.cH1:M11.1..1

alpina $\quad \ldots \quad \ldots \quad$ …

vulearis $\quad \ldots \quad \ldots \quad 7 \quad$ I.XiII

AI.I.1T. II

Scheenoprasum $\quad \ldots \quad 21 \quad$ ccxill.

Alsixi:

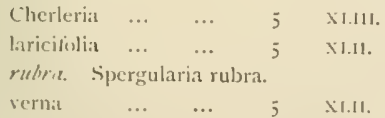

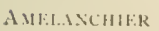

vulgaris $\quad \ldots \quad$... \& L....

AxliROA.IC

Chancejasme $\quad .$. is cxicl.

glacialis $\quad \ldots \quad \ldots$ is cici.

obtusifolia $\ldots \quad \ldots \quad$ is cxit.

\begin{tabular}{|c|c|c|}
\hline ANEMONI: & & Page. \\
\hline alpina & $\ldots$ & 1 \\
\hline sulphurea & alpina var.) & 1 \\
\hline vernalis & ... & 1 \\
\hline
\end{tabular}

Antennerie. (inaphalium.

Anthericum liliastrum. Liliastrum aibum.

ANTHY1.1.15

Vulneraria $\ldots \quad \ldots \quad 6 \quad 6 \quad$ 1.

Aguriticil.

alpinat $\quad \ldots \quad \ldots \quad 2 \quad \ldots \quad \mathrm{xr}$.

atrata iulgaris var.) 2 XI:

ARAlis:

$\begin{array}{lcccc}\text { alpina } & \ldots & \ldots & 2 & \mathrm{xx} . \\ \text { bellidifolia } & \ldots & \ldots & 2 & \mathrm{xx} . \\ \text { ciliata alpestris var.) } & 2 & \mathrm{xx} . \\ \text { citrulea } & \ldots & \ldots & 2 & \mathrm{xix} . \\ \text { Halleri } & \ldots & \ldots & 2 & \mathrm{xx} .\end{array}$

Arbutus Lint-lrsi. Arctostaphylos officinalis.

Arctostaplyos

officinalis ... ... It cxisuts.

Uure-Ursi. officinalis.

ARENARIA

biflora $\quad \ldots \quad \ldots \quad$; 1 xill,

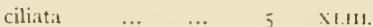

nubra. Spergularia rubra.

verna. Alsine verna.

Aretiu alpince. Androsace glacialis.

ARNIC.

Clusii. Aronicum Clusii.

Doronitum. Aronicum Clusii.

montana $\quad \ldots \quad \ldots \quad 12 \quad \mathrm{Cxx}$.

Aronic. Anelanchier.

Aroniclil

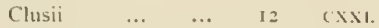

Joronitum. Clusii. 


\section{Index.}

ARTEMISIA

$\begin{array}{lcccc}\text { Mutellina } & \ldots & \ldots & \text { II } & \text { CXVir. } \\ \text { spicata } & \ldots & \ldots & \text { II } & \text { CXVI. }\end{array}$

AsTik

alpinus $\quad \ldots \quad \ldots \quad$ I $\quad$ CXI.

Astregalus alpinus. Phaca astragalina.

Astrugulus australis. Phaca australis.

Astrasalus campestris. Oxytropis campestris.

Astragalus montanus. Oxytropis montana.

Astragalus urulansis. Oxytropis Halleri.

A:TR.INTIA

minor $\ldots$... 10 xerl.

ATR.AGENE alpina

I.

AZALEA procumbens $\quad \ldots \quad 14$ ('Xis.

B.JRTIIA alpina $\quad \ldots \quad \ldots \quad 16 \quad$ CLxill.

BELLIDIASTRLY Michelii $\quad . . \quad \ldots \quad 11 \quad$ CXI.

BEI.I.IS perennis $\quad . . \quad \ldots \quad 11 \quad \mathrm{CXI}$.

BERPERIS vulgaris $\quad \ldots \quad \ldots \quad$ 2 XIX

BisCUTELLA lievigata $\quad \ldots \quad \ldots \quad$... 3 xit. striatilis. levigata.

Brmella. prunella.

BIPIEURUN stellatum $\quad \ldots \quad \ldots \quad$ 10 $\quad \mathrm{xCl}$.

C.I.A.INTHA
alpina

C.Alititriche verna (stagnalis var.) 8 L.xx.xiv.

Callunit vulgaris $\quad . . \quad \ldots \quad 14$ CxLIII.

CALTHA palustris $\quad \ldots \quad \ldots \quad \quad 2 \quad x$ ! I.

C.MMPANUI..

barbata $\quad \ldots \quad \ldots \quad 13 \quad$ CXI.I.

pusilla $\quad \ldots \quad \ldots \quad 13$ CxLIII.

rapunculoides $\quad \ldots \quad$ I3 CXLII.

Scheuchzeri... $\quad . . \quad 13 \quad$ cxlli.

thyrsoiclea $\ldots . . . . \quad$ is

$\begin{array}{ccccc}\text { Cardimine } & & & \text { Page. } & \text { Plate. } \\ \text { alpina } & \ldots & \ldots & 2 & \text { xxi. } \\ \text { amara } & \ldots & \ldots & 2 & \text { xxil. } \\ \text { pratensis } & \ldots & \ldots & 2 & \text { xxII. } \\ \text { resedifolia } & \ldots & \ldots & 2 & \text { xxi. }\end{array}$

CAREX

canescens $\ldots . \quad \ldots \quad 22$ ccxill

curtur. canescens.

echinata $\quad \ldots \quad \ldots \quad 22 \quad$ ccxxil.

flava... $\quad \ldots \quad \ldots \quad 22$ ccxxiv.

lasopina-see note, p. 22.

leporina $\quad . . \quad \ldots . \quad 22 \quad$ ccxill.

limosa $\quad . . \quad \ldots \quad 22$ ccxxill.

$\begin{array}{lllll}\text { nigra } & \ldots & \ldots & 22 & \\ \text { ccxisul. }\end{array}$

pallescens ... $\quad . . \quad 22$ cс.xxir.

$\begin{array}{lllll}\text { panicea } & \ldots & \ldots & 22 & \text { cCxxill. }\end{array}$

stellulutor. echinata.

$\begin{array}{lllll}\text { vesicaria } & \ldots & \ldots & 22 & \\ \text { coxisul. }\end{array}$

CARLINA

acaulis $\quad \ldots \quad \ldots \quad 12 \quad$ CXxill.

subacaulis, acaulis.

Cerastium

$\begin{array}{lllll}\text { alpinum } & \ldots & \ldots & 5 & \text { XLY. } \\ \text { ariense } & \ldots & \ldots & 5 & \text { XLY. } \\ \text { trigynum } & \ldots & \ldots & 5 & \text { XLY. }\end{array}$

CERASL'S

Padus $\quad \ldots \quad \ldots \quad 7$ Lxil.

Chamanerium. Epilobium.

Cherleria sectoides. Alsine Cherleria.

Chrysunthemum-see Leucanthemum.

CHRYSOSPLENIUM

alternifolium $\quad \ldots$ ro $\mathrm{xCl}$.

CIRSILYM

eriophorum ... $\quad . . \quad 12$ cxiv1.

Clematis alpina. Atragene alpina.

Cnicus. Circium.

Cochlearia

saxatilis $\quad \ldots \quad \ldots . \quad 3$ xxit.

Caloglossum aivide. Habenaria viridis.

Colchicus

autumnale $\ldots \quad \ldots \quad 21 \quad$ ccis:

ComARty

palustre $\quad \ldots \quad \ldots \quad 7 \quad$ ixisit. 


\section{Index.}

CONINI.I.IRII Pages Plate bifiliu. Maianthemum bifolium. majalis $\quad . . \quad \ldots 20 \quad 20$ ("C. Polygomatum. Polygonatum vulgare.

COR.11.1.OR:11\%. innata $\quad \ldots \quad \ldots \quad 20 \quad$ (')

\section{COTONEATIEK} vulgaris $\quad \ldots \quad \ldots \quad$ \& ixi.

CREPIS

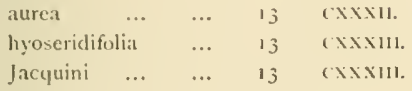

\section{Crocu:}

vernu; $\quad \ldots \quad \ldots \quad 19 \quad$ ixcrill.

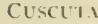

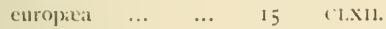

CYIRIPEHLYH Cilceolus $\ldots \quad \ldots \quad$ 20 c(1).

Cinckive. Liliastrum.

D.APHXY:

stritta $\quad \ldots \quad \ldots \quad 18 \quad$ cicrilt.

D)ASTHE:

atrorubens ... ... + .xixil.

deltoides $\quad \ldots \quad \ldots \quad+$ xixill.

superbus $\ldots$... + Xxкil.

sylventris ... ... + хxх11:

Doronicum hirsutum. Aronicum Clusii.

1) R.11:1

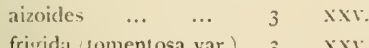

frigida fomentosa var.) 3 x.r.

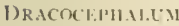
austriacum $\ldots \quad \ldots \quad 17 \quad$ (1.хxхı. Ruyschiana $\quad \ldots \quad 17 \quad$ Clxixi.

1) ROSFIR I longifolia $\ldots \quad \ldots+\quad+$ xixul. rotundifolia... $\ldots .+$ xxixu.

I) RYIS octopetisla $\ldots . \quad \ldots \quad$...

EсHIL' vulgare

CI.xiII.

E.MPETRLY nigrum

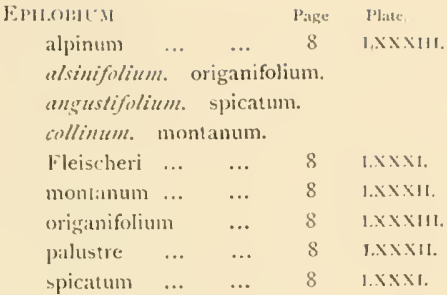

ERIC:I

carneal $\quad . . \quad \ldots \quad$ it cxlitll.

alulguis. Calluna vulgaris.

ERITERON

alpinus $\quad \ldots \quad \ldots \quad$ 11 ( $11 \%$.

drebatchensis (acris var.) II cxil.

uniflorus $\quad \ldots \quad \ldots \quad 11 \quad$ (xil:

Villarsii $\ldots \quad \ldots \quad$ 11 cxilt.

\section{ERIOPHORL'M}

alpinum $\quad \ldots \quad$.. $21 \quad$ CCXNI.

angustifolium $\quad \ldots \quad 21 \quad$ cCxxi.

cafilutum. Sicheuchzeri.

Siheuchzeri $\quad \ldots \quad 21 \quad$ (C)

ERITRICHIL:)

nanum $\quad \ldots \quad \ldots \quad 15 \quad$ (IN)

ERISIMLY

letieticum, rhaticum.

rbeticum $\ldots$... 3 x.xir.

EUPHORI:I

Cyparissias... ... $19 \quad$ (xelill.

EUPHR.ISII

alpina officinalis var.) 16 CIxiı.

minima $\quad \ldots \quad \ldots \quad 16 \quad$ CI.XIII.

FRAR:IRII

vesca

(i.AGE.

fistulosit. L.iottardi.

Liottiurli $\quad \ldots \quad \ldots . \quad 21 \quad$ (C)

Galdus

$\begin{array}{lllll}\text { boreale } & \ldots & \ldots & 10 & \text { c111. } \\ \text { syliestre } & \ldots & \ldots & 10 & \text { c111. } \\ \text { uliginosum } & \ldots & \ldots & 10 & \text { c111. } \\ \text { verum } & \ldots & \ldots & 10 & \text { c11. }\end{array}$


Index.

\begin{tabular}{|c|c|c|c|c|}
\hline & Page. & Plate. \\
\hline \multirow{2}{*}{\multicolumn{2}{|c|}{$\begin{array}{l}\text { GentIANA } \\
\text { asclepiadea ... } \\
\text { bavarica } \quad . .\end{array}$}} & $\ldots$ & 14 & Cl.vill. \\
\hline & & $\ldots$ & 15 & CIIX. \\
\hline \multicolumn{2}{|c|}{ brachyphylla } & ... & 15 & CIX. \\
\hline \multicolumn{2}{|c|}{ campestris ... } & $\ldots$ & 15 & cl.si. \\
\hline \multicolumn{2}{|c|}{ Charpentieri } & ... & 14 & Ci.: \\
\hline ciliata & $\ldots$ & ... & 14 & c1.1x. \\
\hline cruciata & $\cdots$ & ... & I4 & CLYII. \\
\hline excisa & $\ldots$ & $\ldots$ & 14 & CI.I. \\
\hline germanica & $\ldots$ & $\ldots$ & 15 & CIAI. \\
\hline \multicolumn{5}{|c|}{ tenella. } \\
\hline lutea ... & $\ldots$ & ... & 14 & CIIS. \\
\hline nivalis & $\ldots$ & ... & 15 & CIX. \\
\hline obtusifolia & $\ldots$ & ... & 15 & CIXI. \\
\hline punctata & ... & ... & 14 & CLWI \\
\hline tenella & $\ldots$ & $\ldots$ & 15 & CLXI. \\
\hline utriculosa & $\ldots$ & ... & 15 & C1X. \\
\hline verna & $\ldots$ & $\ldots$ & 15 & CLX. \\
\hline
\end{tabular}

GERINIUM

aconitifolium $\quad \ldots \quad 5$ Xivil.

sylvaticum $\ldots \quad \ldots \quad 5 \quad$ xLir.

GELTI

$\begin{array}{lllll}\operatorname{montanum} & \ldots & \ldots & 7 & \text { LXXi. } \\ \text { reptans } & \ldots & \ldots & 7 & \text { IXX. } \\ \text { rivale } & \ldots & \ldots & 7 & \text { Lxix. }\end{array}$

GLOBULARIA

cordifolia $\quad \ldots \quad \ldots \quad$ Is $\quad$ cxcill.

GNAPH.11,IUM

\begin{tabular}{|c|c|c|c|}
\hline carpathicum & $\ldots$ & I 1 & Cxve \\
\hline dioicum & $\ldots$ & II & $\mathrm{CXl}$ \\
\hline $\begin{array}{l}\text { Leontopodiun } \\
\text { pusillum. }\end{array}$ & $\ldots$ & II & cxit. \\
\hline ipinum & $\cdots$ & I I & cxi. \\
\hline ylvaticum .. & $\ldots$ & II & $\mathrm{CXV}$. \\
\hline
\end{tabular}

GMNADENIA

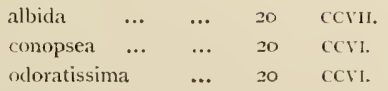

GipsophILA

repens $\quad \ldots \quad$... 4 xxir.

H.MIIN.IRIA

viridis $\quad \ldots \quad \ldots \quad 20 \quad$ Cevil.

HEINSARUMI

oloscurum $\quad \ldots \quad$... 6 1.xr.
Heliaxtheyum Page Phate.

alpestre. celandicum.

celandicum ... ... 3 xxv11.

vulgare $\quad \ldots \quad \ldots \quad 3$ xxvil.

HERXIARIA

glabra $\quad \ldots \quad \ldots \quad 8 \quad$ 1.xxiv.

HiERTCIUM

albidum $\quad \ldots \quad \ldots \quad 1_{3}$ cxixix.

alpinum $\quad \ldots \quad \ldots \quad$ I3 Cxxivi.

aurantiacum $\quad$... 13 cxxisv.

Auricula $\quad . . \quad \ldots \quad$ is cxixiv.

chondrilloides. Crepis Jacquini.

dentatum (villosum var.) 13 cxxist.

glanduliferun $\quad \ldots$ I3 cxxxv.

intybaccum. albidum.

pilosella $\quad . . \quad \ldots \quad$ I 3 cxxiv.

pilloselliforme (Pilo-

sclla var.) $\quad \ldots \quad 13$ cxxiv.

scorzonerifolium $\quad \ldots \quad$ I3 cxxxvit.

HIPPOCREPIS

comosal $\quad \ldots \quad \ldots \quad 6 \quad 6 \quad$ I.XIII.

Howorive

alpina $\quad \ldots \quad \ldots \quad$ I

HORMINUM

pyrenaicum ... ... $17 \quad$ сг.хх.

HUTCHIXSIA

$\begin{array}{lllll}\text { alpina } & \ldots & \ldots & 3 & \mathrm{x} \% \text {. }\end{array}$

HYPERICUA

quadrangulum $\quad \ldots \quad 5 \quad$ xis'.

dubium. quadrangulum.

HYPOCH.ERIS

zmiflora $\quad \ldots \quad \ldots \quad 12 \quad$ cxix.

JUNCL'S

\begin{tabular}{|c|c|c|c|c|}
\hline alpinus & $\ldots$ & $\ldots$ & 21 & CC.NYJI \\
\hline filiformis & $\ldots$ & $\ldots$ & $2 \mathrm{I}$ & cCxvtll. \\
\hline Jacquini & $\ldots$ & $\ldots$ & 21 & c CX1X. \\
\hline trifidus & $\ldots$ & $\cdots$ & 21 & ccxix. \\
\hline riglumis & $\ldots$ & $\ldots$ & 21 & coxix. \\
\hline
\end{tabular}

kernerr. Cochlearia.

KN.ALTI.)

arrensis $\quad \ldots \quad \ldots$ II CVII.

LAMIUTI

album $\quad \ldots \quad \ldots \quad$ I7 crixxit. 


\section{Index.}

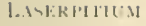

hirsutum Plase. Plate.

Pmalt: hirsutum.

I.. TIITRE

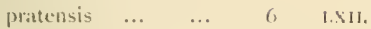

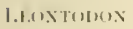

hispidus $\quad \ldots \quad$... 12 (xхx1.

hrestilis. hispidus.

leontopotium alpinun. Cinaphalium l.contopodium.

lofisonum. Spergularia.

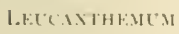

:1ри1иm $\quad \ldots \quad$... 12 ixvil.

ceratophỵlloides-sec note, p. 12.

cormopifolium ... 12 cxill.

rulsate $\quad \ldots \quad \ldots \quad 12 \quad$ (xvil.

1.11.1.1-TRT.

allom $\quad \ldots \quad \ldots \quad 21 \quad$ cexil:

1.11.11. V

bulbiferum $\ldots \quad$... $20 \quad$ crxi.

Martagon $\ldots \quad$... $20 \quad$ CCX.

L.IX.Iki.

alpinat $\quad \ldots \quad \ldots \quad 15 \quad$ CLXVI.

rulgaris $\quad . . \quad \ldots \quad 15$ chivi.

1.1.x. . .

besealis $\quad . . \quad$... 10 (II.

I.I.()) 111.1

serotina $\quad \ldots \quad \ldots . \quad 21 \quad$ ('Nit.

Loiseleuriz. Azalea.

1. (N.) IC I.R.)

alpigenal $\quad \ldots \quad$... $10 \quad$ (1.

cicrulea $\quad \ldots \quad$... 10 cil.

nignia $\quad \ldots \quad \ldots$ ro

I.rTt-

corniculatus $\quad \ldots$ 6 6 T.III.

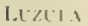

lutea... $\quad \ldots \quad \ldots \quad 21 \quad$ icxi.

niveil $\quad \ldots \quad \ldots \quad 21 \quad$ cexis.

(.) (н):

alpina $\quad \ldots \quad \ldots \quad ;$ xi.

dievic: Melandrium sylvestre.

diumu. Melandrium syliestre.

Flos-Cuculi ... ... ; Xi..

Flus-Jovis $\quad \ldots \quad$... $\quad 5 \quad$ xi.l.

Mrestris. Melandrium sylvestre.

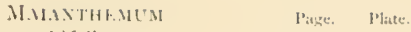

$\begin{array}{llllll}\text { bifolium } & \ldots & \ldots & 20 & \text { (C) }\end{array}$

Metule:ic)

fillata $\quad \ldots \quad \ldots 6 \quad 6 \quad 1$.

MrI.MMURTM

pratense $\quad \ldots \quad$.. 16 (16 exilli.

sylvaticum $\ldots \quad \ldots \quad 16 \quad$ (1x)ा1

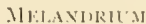

spliestre $\quad \ldots \quad \ldots \quad+$ ixix.

IrLuturts

arvenis $\quad \ldots \quad \ldots \quad$ G 1.1 .

officinalis. arvensis.

Mespilus Amelanthier. Amelanchier vulgaris.

Mesprilus Cofonenster. Cotoneaster vulyaris.

. $\mathrm{NE: \textrm {L } ^ { \prime } \mathrm { M }}$

Mucllina $\ldots \quad \ldots \quad 10$ xсvir.

Monese's grendiffore. Pyrola uniflora.

MoN111

rivularis (fontana var.) 8 I.X.x.

Mrontris

alpestris $\quad \ldots \quad$... $15 \quad$ c1.NIs:

notur. Eritriclium nanum.

MYRIC.IRIS

germanica $\ldots \quad \ldots \quad \& \quad$ ixixil:

NisTLRTLITI

palustre $\quad \ldots \quad \ldots \quad 3 \quad 3 \quad$ ximi.

tirrestre. palustic.

NITIRITLILS

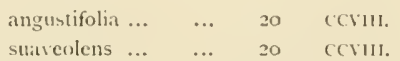

O.NOI:RICHI:

montana $\quad . .6 \quad \ldots \quad 6 \quad$ IXIV.

ONONIS

rotundifolia... $\quad . .6 \quad 6 \quad$ xı.

ORCHIS

allida. Gymnadenia albida.

conopsea. Giymnadenia conopsea.

globona $\quad \ldots \quad$... $20 \quad$ cill.

latifolia $\quad \ldots \quad \ldots \quad 20 \quad$ (C)

maculata $\ldots . \quad \ldots \quad 20 \quad$ CCl.

mascula $\quad \ldots \quad \ldots \quad 20 \quad$ (c)

nigra. Nigritella angustifolia.

oderatissimar. Gymnadenia odoratissima. suretedens. Nigritella suat eolens.

sivitis. Habenaria viridis. 


\section{Index.}

OxALIS

Acetosella $\ldots \quad \ldots \quad \ldots \quad 5 \quad$ XI.IX.

Oxroocers

palustris $\quad \ldots \quad$... $14 \quad$ CI.I.

OxITROPI

$\begin{array}{lllll}\text { campestris } & \ldots & \ldots & 6 & \text { I.VII. } \\ \text { Halleri } & \ldots & \ldots & 6 & \text { I.VII. } \\ \text { lapponica } & \ldots & \ldots & 6 & \text { I.IX. } \\ \text { montana } & \ldots & \ldots & 6 & \text { I.VIII. } \\ \text { uralensis. } & \text { Halleri. } & & \end{array}$

\section{PAPAVER}

tlaviflorum (alpinum var.) 2 xx.

pyrentizun. flaviflorum.

Paralisia. Liliastrum.

PARIS

quadrifolia...$\quad \ldots \quad 20 \quad$ ecit.

PARNASSI.I

palustris $\ldots+\ldots+$ xxxı.

PEDICULARIS

$\begin{array}{lllll}\text { atro-rubens } & \ldots & \ldots & 16 & \text { Clxxil. } \\ \text { foliosa } & \ldots & \ldots & 16 & \text { CLxxil. } \\ \text { incarnata } & \ldots & \ldots & 16 & \text { CLxxi. } \\ \text { palustris } & \ldots & \ldots & 16 & \text { CLxxi. } \\ \text { recutita } & \ldots & \ldots & 16 & \text { CLxxiv. } \\ \text { rostrata } & \ldots & \ldots & 16 & \text { CLxx. } \\ \text { tuberosit } & \ldots & \ldots & 16 & \text { CLxxi. } \\ \text { verticillata } & \ldots & \ldots & \text { I6 } & \text { CLxxv. }\end{array}$

PHACA

$\begin{array}{lllll}\text { alpina } & \ldots & \ldots & 6 & \text { L.IV. } \\ \text { astragalina } & \ldots & \ldots & 6 & \text { LVII. } \\ \text { australis } & \ldots & \ldots & 6 & \text { I.VI. } \\ \text { frigrida } & \ldots & \ldots & 6 & \text { LV. }\end{array}$

PHITETMA

betonicifolium $\quad \ldots \quad 13$ cxIvI.

Halleri $\quad \ldots \quad \ldots \quad 13 \quad$ cxivis.

hemisphericum $\quad . .13$ cxi.Iv.

humile $\quad \ldots \quad \ldots \quad 13$ cxulv.

orbiculare ... $\quad . . \quad$ I3 CXLV.

pauciflorum... $\quad . . \quad$ I3 CXIIV.

Scheuchzeri $\quad$.. 13 cxuv.

spicatum var. nigrum-see note p. 13.

Pinguicutal

alpina $\quad \ldots \quad \ldots \quad$ I7 $\quad$ Cl.xxxiv.

grandiflora ... $\quad . . \quad 17$ Cr.xxxiv.

vulgaris $\quad \ldots \quad$... 17 crxxxiv.

PlanTuio

alpinat

I8

CXCIII.
POLEMONIL: Page. Plate.

croleum $\quad . . \quad \ldots \quad 15 \quad$ CLXiI.

Polyfill.

amara $\quad \ldots \quad \ldots+\quad+$ xximil.

Chammbuxus $\quad . .4$ xxinl.

POLIGONATUM

officinule. vulgare.

vulgare $\quad \ldots \quad \ldots 20$ cxcix.

Polygonum

amphibium ... $\quad \ldots \quad 18 \quad \mathrm{cxcV}$.

Bistortil $\ldots$... I8 cxciv.

vivipartum $\quad \ldots \quad \ldots \quad 18$ Cxciv.

POTENTIS.I.I

alpestris. maculata.

Anserina $\quad . . \quad \ldots \quad 7$ I.xxII.

argentea $\ldots \quad \ldots \quad 7$ Lxxili.

atrea $\quad \ldots \quad \ldots \quad 7$ Lxxil,

caulescens ... ... 7 1.xxv.

Comırum. Comarum palustre.

firma (maculata var.) 7 Ixxiv.

grandiflora ... $\quad . . \quad 7$ Lxxv.

maculata see firma.

sabuludu. firma.

salisburgensis. maculata.

Tomentilla $\quad \ldots \quad 7$ LxxvI,

vernal $\quad \ldots \quad \ldots \quad 7$ I.xiIII.

PrIMULA

Candollema. integrifolia.

dinjana $\quad \ldots \quad \ldots \quad 17 \quad \mathrm{CxC}$

$\begin{array}{lllll}\text { elatior } & \ldots & \ldots & \text { I7 } & \text { CLxxxvi. }\end{array}$

farinosa $\quad \ldots \quad \ldots \quad$ I7 CLxxxull.

glutinosa $\quad \ldots \quad \ldots . \quad 17 \quad$ Cxc.

graveolens ... $\quad . . \quad 17 \quad$ cixixix.

hirsutu. viscosa.

integrifolia ... $\quad \ldots \quad$ I7 Cxc.

latifolia. graveolens.

longiflore $\quad . . \quad \ldots \quad 17 \quad$ CLXXxvin.

Wuretiunc. dinyana.

officinalis $\ldots \quad \ldots \quad 17 \quad$ CLxxxy.

zeris. officinalis.

villost. viscosa.

viscosit $\quad . ., \ldots .17 \quad$ cxc.

PRUNELI

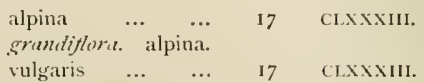

P'runus Pudus. Cerasus Padus.

Ptarmica. Achillea. 
Index:

l't H.NON.IRIA H'uge, Plate, angustifolor. azurea. itzurea $\quad \ldots \quad \ldots \quad 15 \quad$ C1.

l'ulsafiller. Anemone.

l'y'sthrum-sce I.eucanthemum.

l'y kot.

\begin{tabular}{|c|c|c|c|c|}
\hline minor & $\cdots$ & • & 14 & CIIII. \\
\hline rotundifoliat & ... & ... & 14 & ('I.II. \\
\hline secunda & & ... & 14 & C.1.111. \\
\hline itloris & & ... & 14 & Cl.111. \\
\hline
\end{tabular}

Pukts aucuparia $\ldots \quad$... 8 I.xxix.

R.INGNCLUS

aconitifolius $\quad$... $\quad 1 \quad$ VIr.

alpestris $\quad \ldots \quad \ldots \quad \quad 1 \quad$ VI.

ayuntilis-sce capillaceus.

Cipillaceus ... ... I $\quad$ I

ylacialis $\quad \ldots \quad$... I $\quad$ II.

hybridus $\quad \ldots \quad$... I

montanus $\ldots \quad$... $\quad$ l $\quad$ x.

parnassifolius $\quad . . \quad$ l vill.

pyrencus $\quad \ldots \quad$... 1 vil.

repens $\quad \ldots \quad$... I

replans (Flammula var.) $t$ IX.

Thora $\quad \ldots \quad$... I 1 I.

k'hapontioum scariosum. Serratula Rhaponticum.

\section{RHIX.NTHE'S}

Crista-Girlli, minor.

major $\quad \ldots \quad \ldots \quad 16 \quad$ CLXix.

minor $\quad \ldots \quad \ldots \quad 16 \quad$ (1.xix.

RHUIOUF.XDRON

ferrugineum $\quad$.. 14 CXIIS:

hirsutum $\quad . .6 \quad \ldots$ if (xilx)

RII:Fs

alpinum $\quad \ldots \quad \ldots \quad 9 \quad$ I.XXsix

petratum-sce note p. 9 .

rubrum $\quad \ldots \quad \ldots \quad 9 \quad$ I.x.xix.

Ro-

:lpina $\quad \ldots \quad \ldots \quad 7 \quad$ I.xxvill.

Rines:

Idaus $\quad \ldots \quad \ldots \quad 7 \quad$ I.xxillI.

Swisis

I.innivi $\quad \ldots \quad \ldots \quad ; \quad$ XLII.

SIIIX

reticulata $\quad . . \quad \ldots \quad$ is cxcitit.

S.II.YII

pratensis $\quad . . \quad \ldots \quad 16 \quad$ cLxix.

\begin{tabular}{|c|c|c|c|c|}
\hline $\begin{array}{l}\text { Simbects } \\
\text { bibulus } \\
\text { racemosa }\end{array}$ & $\begin{array}{l}\ldots \\
\ldots\end{array}$ & $\begin{array}{l}\ldots \\
\ldots\end{array}$ & $\begin{array}{l}\text { Jage: } \\
10 \\
10\end{array}$ & $\begin{array}{l}\text { Plate. } \\
\text { XCix. } \\
\text { (. }\end{array}$ \\
\hline $\begin{array}{c}\text { S.AxuI ISOKB.I } \\
\text { ofticinalis }\end{array}$ & $\ldots$ & $\ldots$ & 7 & I.xili. \\
\hline $\begin{array}{l}\text { S.POXIKIA } \\
\text { ocymoides }\end{array}$ & $\ldots$ & $\ldots$ & 4 & xxxy. \\
\hline $\begin{array}{c}\text { SAtSSERTII } \\
\text { alpinat }\end{array}$ & $\ldots$ & $\ldots$ & 12 & (XxพנI1. \\
\hline $\begin{array}{r}\text { SAXIRRM(i, } \\
\text { aimoides }\end{array}$ & $\ldots$ & $\ldots$ & 9 & xell. \\
\hline Aizoon & $\ldots$ & $\ldots$ & 9 & ic. \\
\hline androsacea & $\ldots$ & ... & 9 & xси1. \\
\hline asperat & $\ldots$ & $\ldots$ & 9 & $x(t)$ \\
\hline bryoides (as & spera & var.) & 9 & xicl. \\
\hline$r \cdot s \cdot s i a$ & $\ldots$ & $\ldots$ & 9 & ic: \\
\hline cuneifolia & $\ldots$ & $\ldots$ & 9 & xill. \\
\hline exaratia & $\ldots$ & $\ldots$ & 9 & xilli. \\
\hline oppositifolia & & $\ldots$ & 9 & ic: \\
\hline planifolia & $\ldots$ & $\ldots$ & 9 & Xicili. \\
\hline rotundifolia & $\ldots$ & $\ldots$ & 9 & xс⿰丿. \\
\hline Seguieri & ... & $\ldots$ & 9 & xcill. \\
\hline stellaris & $\ldots$ & $\ldots$ & 9 & $\mathrm{xc}$. \\
\hline
\end{tabular}

Sicinosil

(1) z'ensis. Knautia arvensis. lucida (Columbaria var.) 11

CYIII.

SclikiYTHUS

:nnuแs $\quad \ldots \quad \ldots \quad$ \&

Sint:

itcre $\ldots \quad \ldots \quad \ldots \quad 9 \quad$ Lxxxis.

album $\quad \ldots \quad \ldots \quad 9 \quad$ I.xхxн.

alpestre. repens.

innum $\quad \ldots \quad$... 9 1.xхx:

atratum $\quad \ldots \quad$...

disyphyllum $\quad$... 9 1.x.xis

repens $\quad \ldots \quad$.. $\quad 9 \quad$ Lxxixt.

saratile. repens.

villosum $\quad \ldots \quad$... $\quad 9 \quad$ I.XXXv.

SEMIRKTIUM

irachnoideum $\quad$... 9 Ixxxvil.

tectorum $\quad \ldots \quad$... $\quad 9 \quad$ IXxixil.

IVulfeni $\quad \ldots \quad \ldots .99 \quad 9 \quad$ I.xxxil.

SENTCO

abrotanifolius $\quad \ldots \quad 12 \quad$ ('xixt.

carniolicus (incanus var.) 12 ('XXi.

1)orunicum ... $\quad . . \quad 12 \quad$ (Xxil.

Fuchsii $\quad \ldots \quad \ldots \quad 12 \quad$ (XXIV.

nebrodensis $\quad \ldots \quad 12$ cxim.

vincusus $\quad \ldots \quad \ldots \quad 12 \quad$ (xil. 
Index.

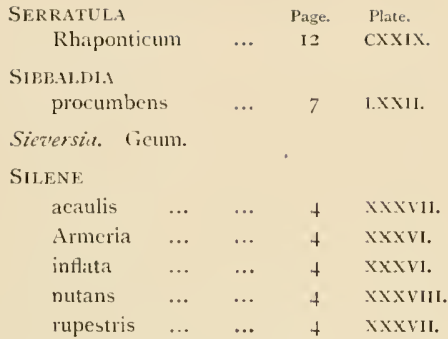

Smilacina. Maianthemum.

SOLDANELLA

$\begin{array}{lllll}\text { alpina } & \ldots & \ldots & \text { is } & \text { сxси. } \\ \text { pusilla } & \ldots & \ldots & \text { is } & \text { cxicu. }\end{array}$

\section{Solwdir)}

Virga-aurea $\quad . . \quad 11 \quad \mathrm{cx}$.

Sorbus aucupurit. Pyrus aucuparia.

Spergula siginoides. Sagina Linnei.

SPERGULARIA

$\begin{array}{lllll}\text { rubra } & \ldots & \ldots & 5 & \text { xill. }\end{array}$

STELLARIA

cerastoides. Cerastium trigynum.

nemorum $\ldots \quad \ldots \quad 5 \quad$ Xiv.

Tamarix-see Myricaria.

TARAXACUM

palustre (officinale var.) I3 cxxxul.

TEUCRJIII

montanum ... $\ldots \quad$ i7 CLxxxilf,

THAICITRUI aquilegifolium $\quad \ldots \quad$ I II. minus $\quad \ldots \quad \ldots \quad 11$ 11.

THESIUN

alpinum $\quad \ldots \quad \ldots \quad$ is cxevi.

pratense $\quad \ldots \quad \ldots \quad 18 \quad$ cxcvi.

THYML:S

Serpyllum $\ldots \quad$... $16 \quad$ CLxix.

TOFIELIHA

borealis. palustris.

calyculata $\ldots \quad \ldots \quad 21 \quad$ ccxis.

palustris $\quad \ldots \quad \ldots \quad 21 \quad$ CCxvı.

Tormentillix. Potentilla.

TRA;OPOMON

pratensis $\quad \ldots \quad \ldots \quad 13 \quad$ cxxixl.

Tricherr. Knatutia.

TRIFOLILAI Page. Plate.

$\begin{array}{lllll}\text { alpinum } & \ldots & \ldots & 6 & \text { I.II. }\end{array}$

$\begin{array}{lllll}\text { badium } & \ldots & \ldots & 6 & \text { LJl. }\end{array}$

TRlGLOCHIN

palustre $\quad \ldots \quad$... 19 cхcr.

TROLLITS

europieus $\quad \ldots \quad \ldots \quad \quad 2 \quad$ xil.

Tussilugo alpinu. Homogyne alpina.

VACCINIUAS
Mjrtillus $\quad \ldots \quad$... $\quad$ it $\quad$ CI.
oxycocizs. Oxycoccus palustris.
uliginosum $\ldots \quad$... $\quad$ I4 CII.
Vitis-idea $\ldots$... $\quad 14$ CLI.

VALERIANA

$\begin{array}{lllll}\operatorname{montana} & \ldots & \ldots & \text { I I } & \text { CV. } \\ \text { officinalis } & \ldots & \ldots & \text { II } & \text { CIV. } \\ \text { tripteris } & \ldots & \ldots & \text { II } & \text { CVI. }\end{array}$

VER.ATRLY

album $\quad \ldots \quad \ldots \quad 21 \quad$ cCxill,

VERPASCLY

pulverulentum ... I5 cr.xv.

VERONIC:A

\begin{tabular}{|c|c|c|c|}
\hline alpinat & $\ldots$ & 16 & exxivil. \\
\hline Beccabungat & $\ldots$ & 16 & CLXXII. \\
\hline bellidioides ... & $\ldots$ & 16 & cIxxทI. \\
\hline Chamiedrys & .. & 16 & CLXXVI. \\
\hline officinalis & $\ldots$ & 16 & ctxixti. \\
\hline saxatilis & $\ldots$ & 16 & coxival. \\
\hline serpyllifolia ... & $\ldots$ & 16 & CLXXVIII. \\
\hline spicata & $\ldots$ & 16 & CIXXitis. \\
\hline
\end{tabular}

VICla

$\begin{array}{lllll}\text { Cracca } & \ldots & \ldots & 6 & \text { I.X.I. }\end{array}$

$\begin{array}{lllll}\text { sepium } & \ldots & \ldots & 6 & \text { I.X. }\end{array}$

VIOI.A

alpestris (tricolor var.) 3 xxix.

ambigzu. Thomasiana.

biflora $\quad \ldots \quad \ldots . \quad 3$ xix

$\begin{array}{lllll}\text { calcarata } & \ldots & \ldots & 3 & \text { xixi. }\end{array}$

canina $\quad \ldots \quad \ldots \quad 3$ xivill.

flava (calcarata var.) 3 x.xi.

mirabilis $\quad \ldots \quad \ldots \quad 3 \quad \mathrm{xxx}$.

palustris $\quad \ldots \quad \ldots \quad 3$ xхтाl.

pinnata $\quad \ldots \quad \ldots \quad 3$ xxill

saxatilis. alpestris.

Thomasiana $\quad \ldots \quad 3$ xix

Zoysii. flava.

Viscaria alfinc. Lychnis alpina.

Vulneraria. Anthyllis. 


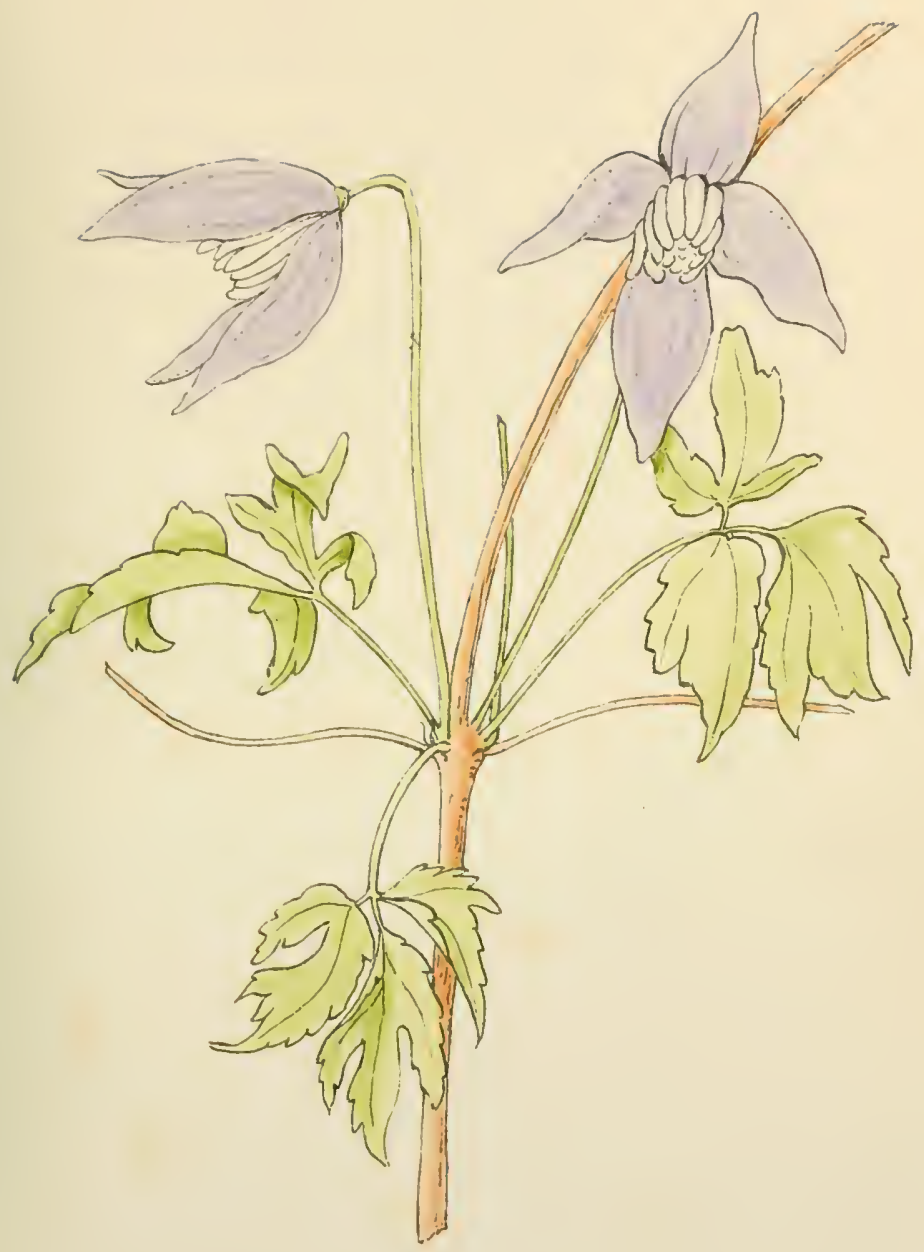

Atragene alpiua. 

II.

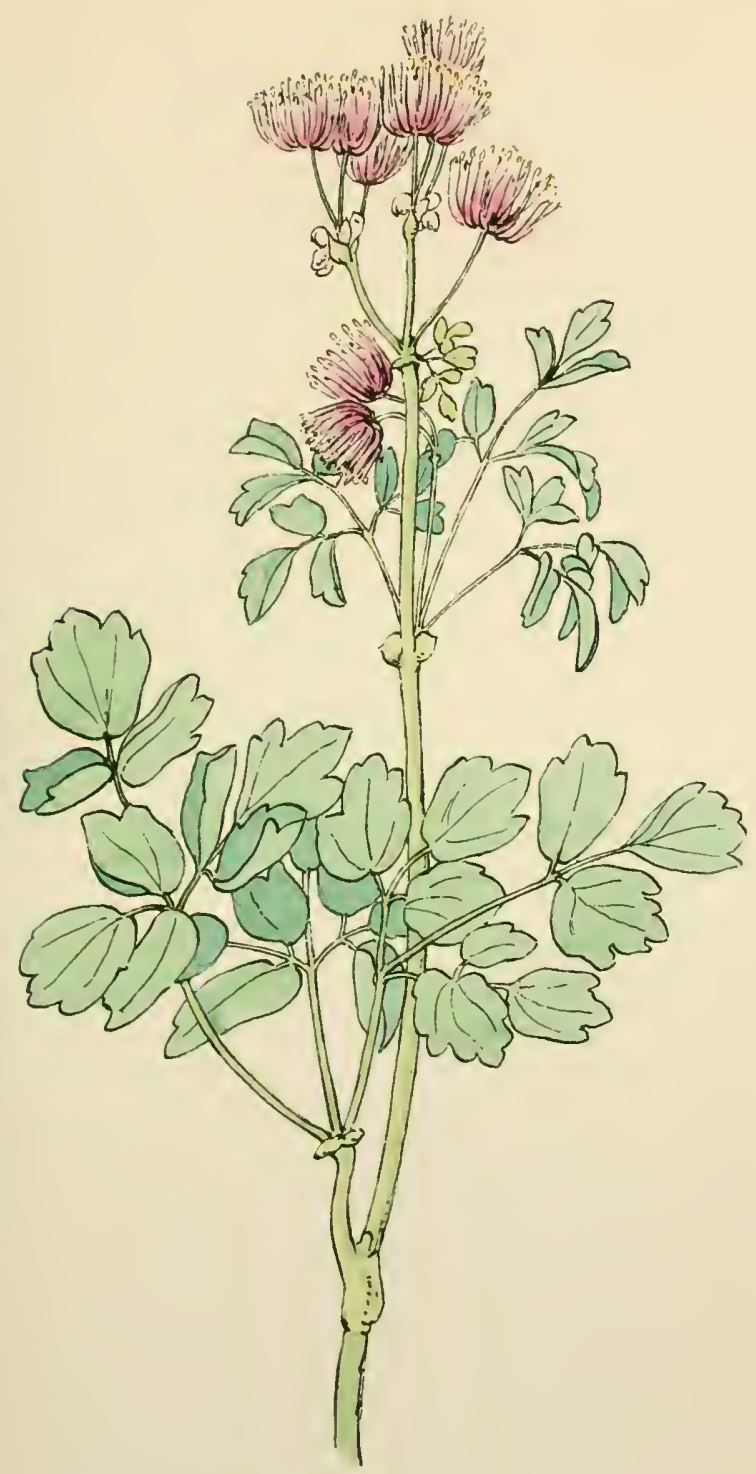

Thalictrum aquilegifolium. 



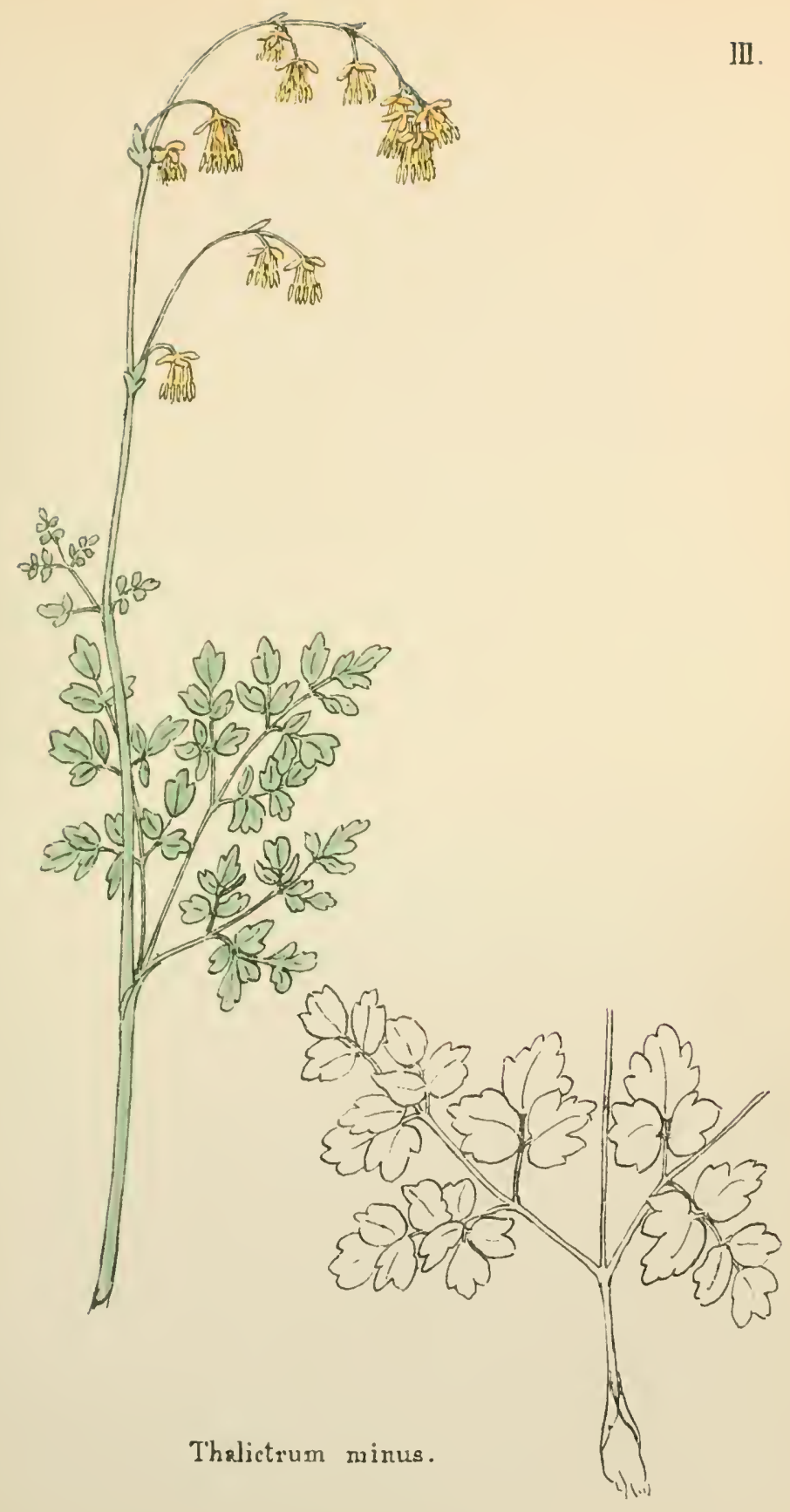


IV.

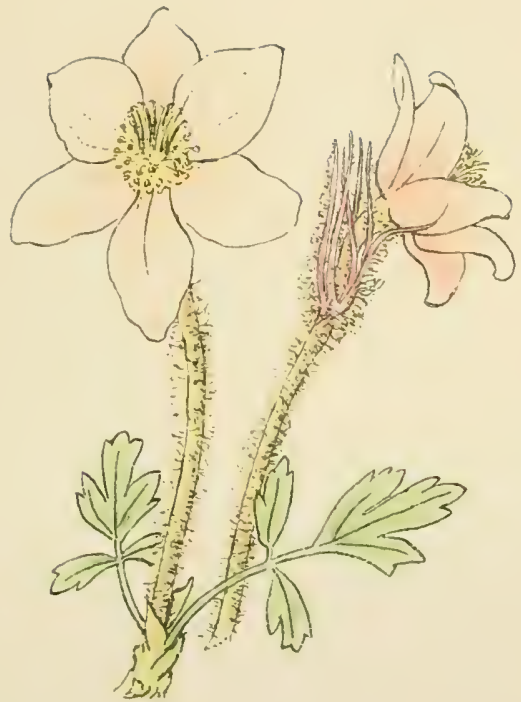

Anemone vernalis. 


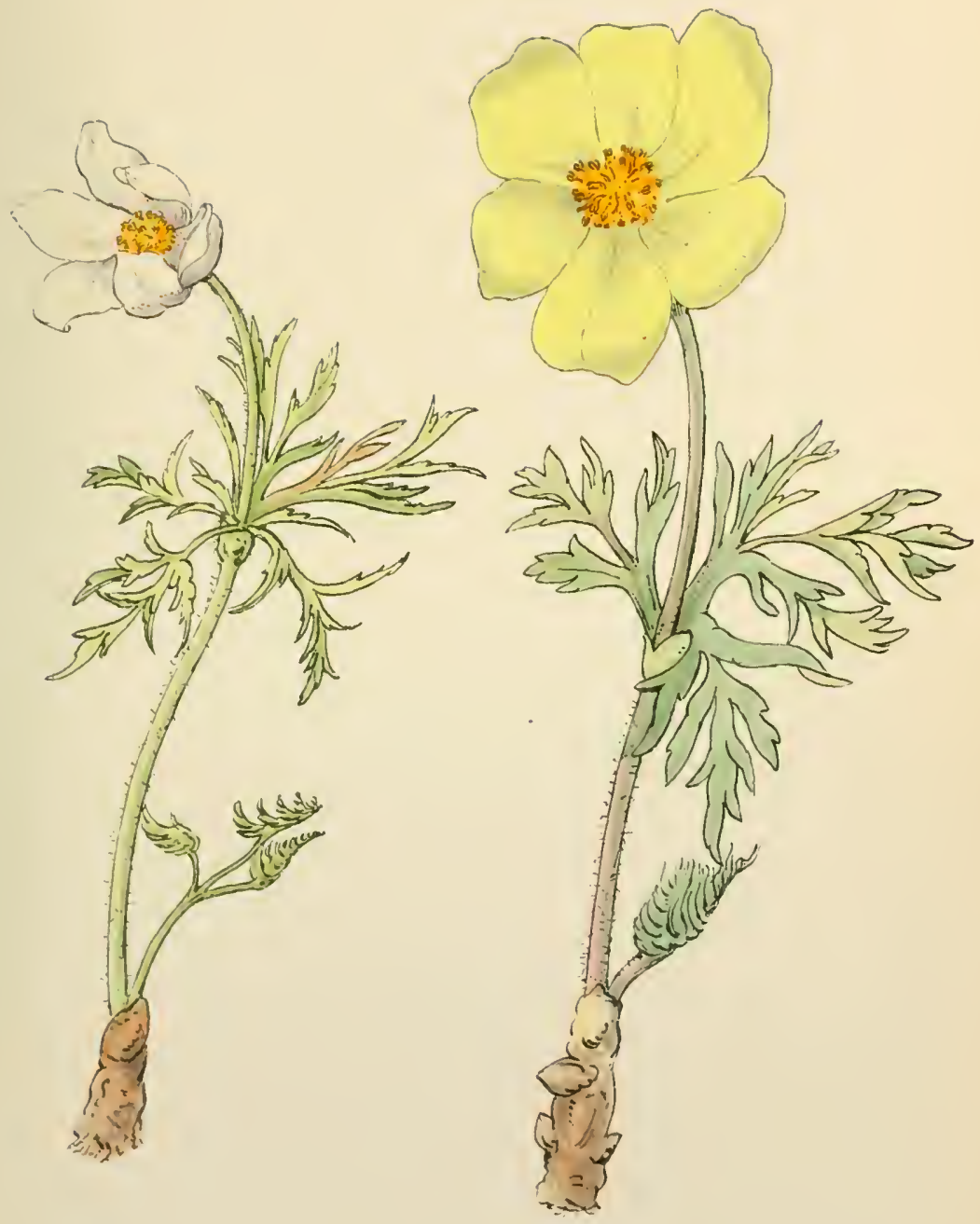

Anenone alpira.

Anemone sulphurea. 



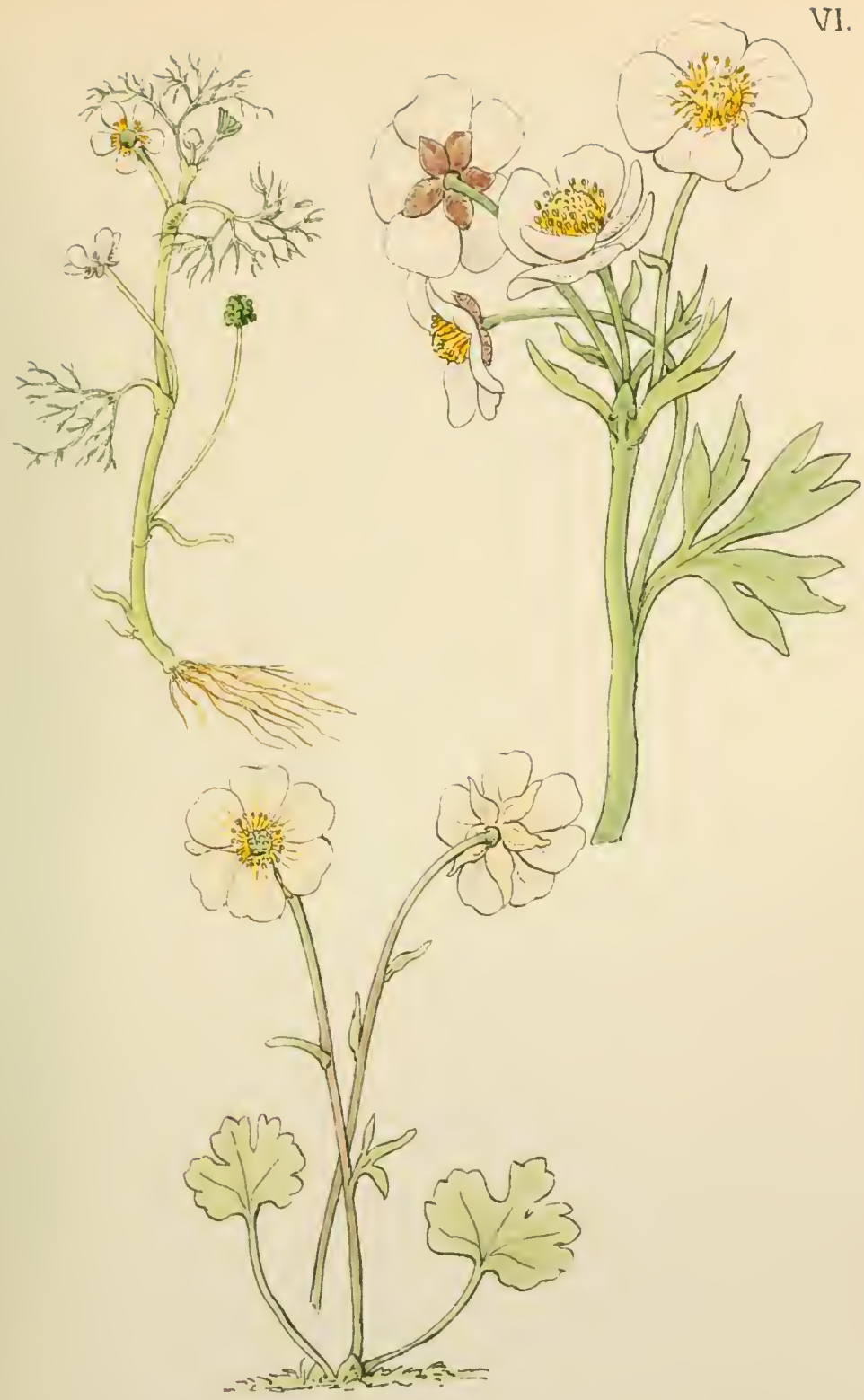

Ranunculus capillaceus.

Ranunculus glacialis.

Ranunculus alpestris. 



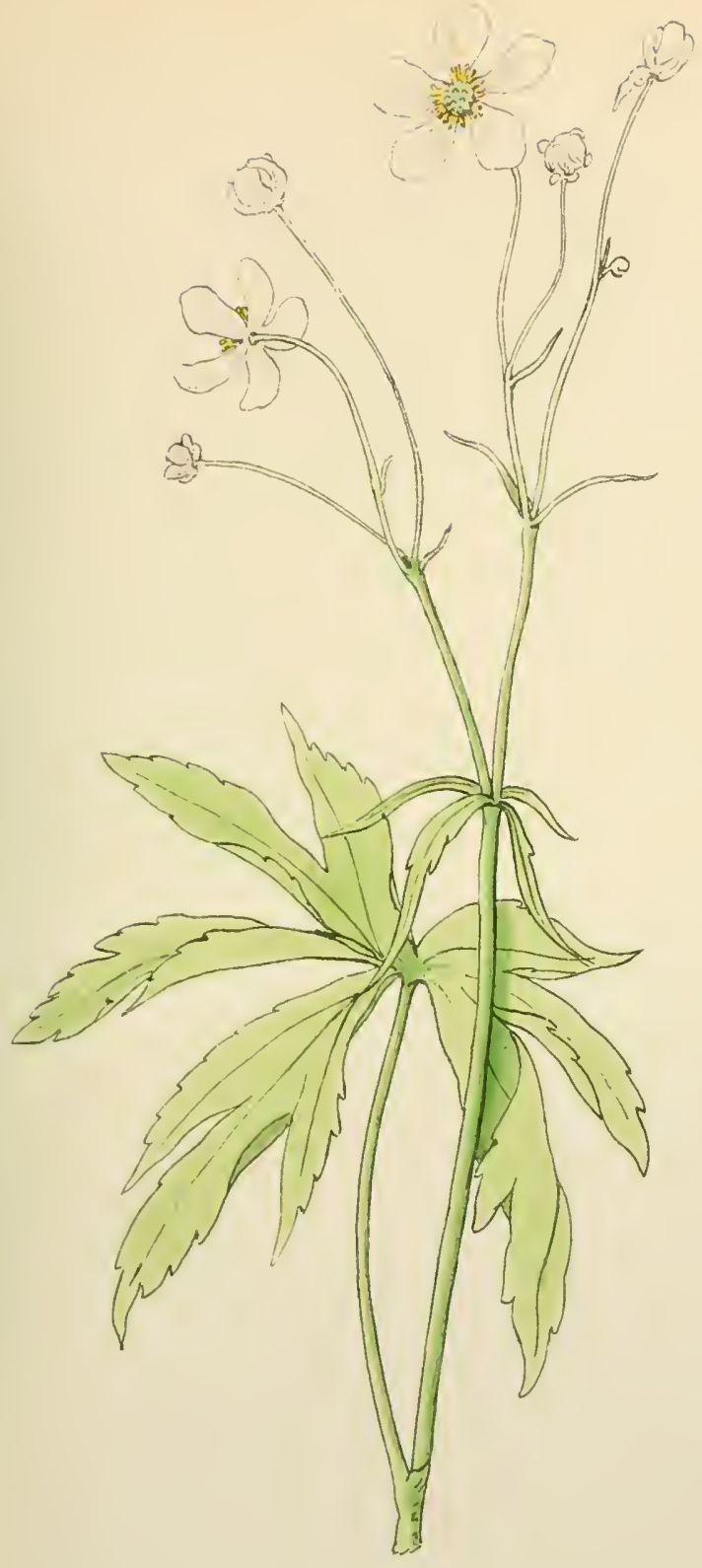

VII.

Ranunculus aconitifolius. 

VIII.
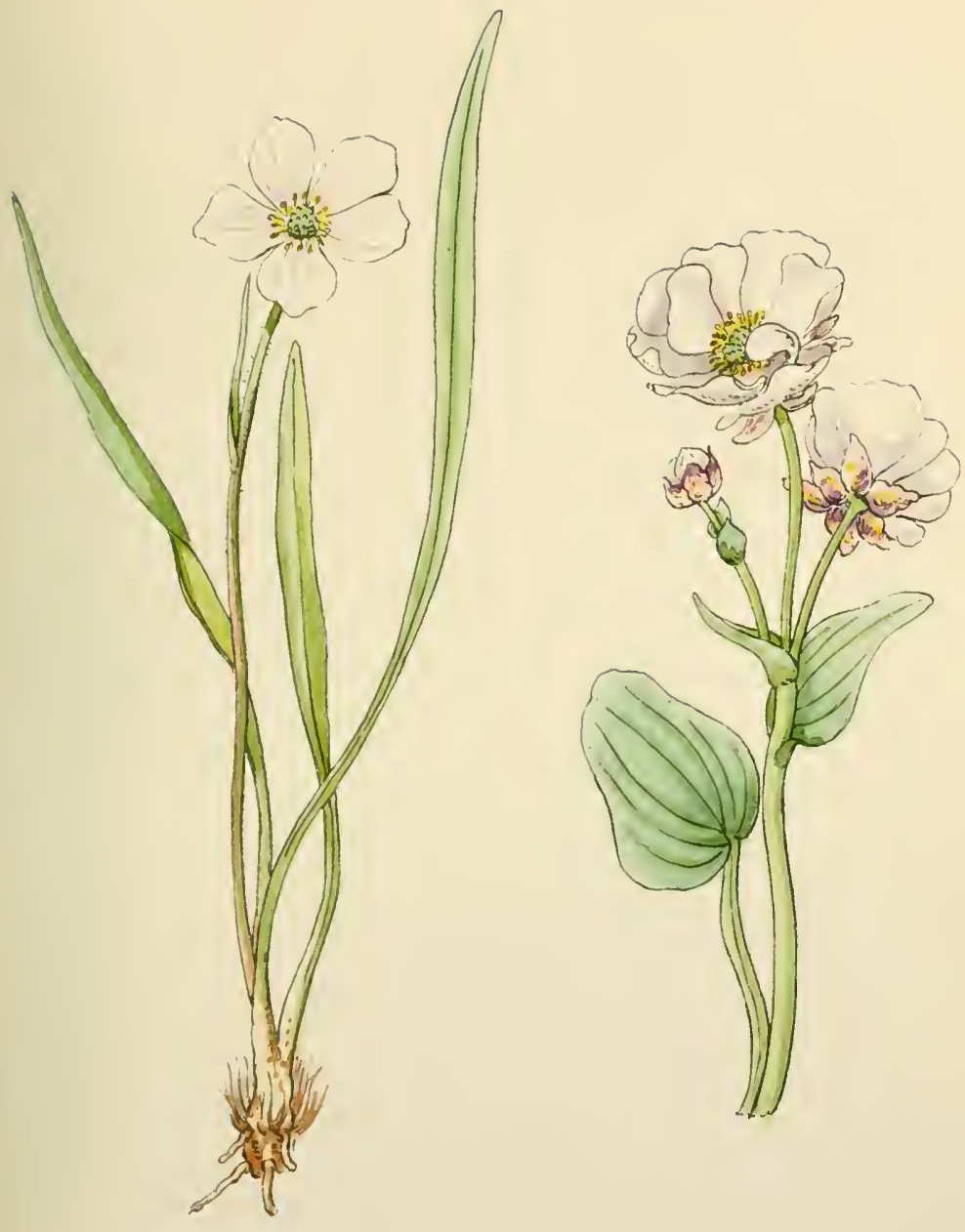

Ranunculus pyrenceus.

Ranunculus parnassifolius. 


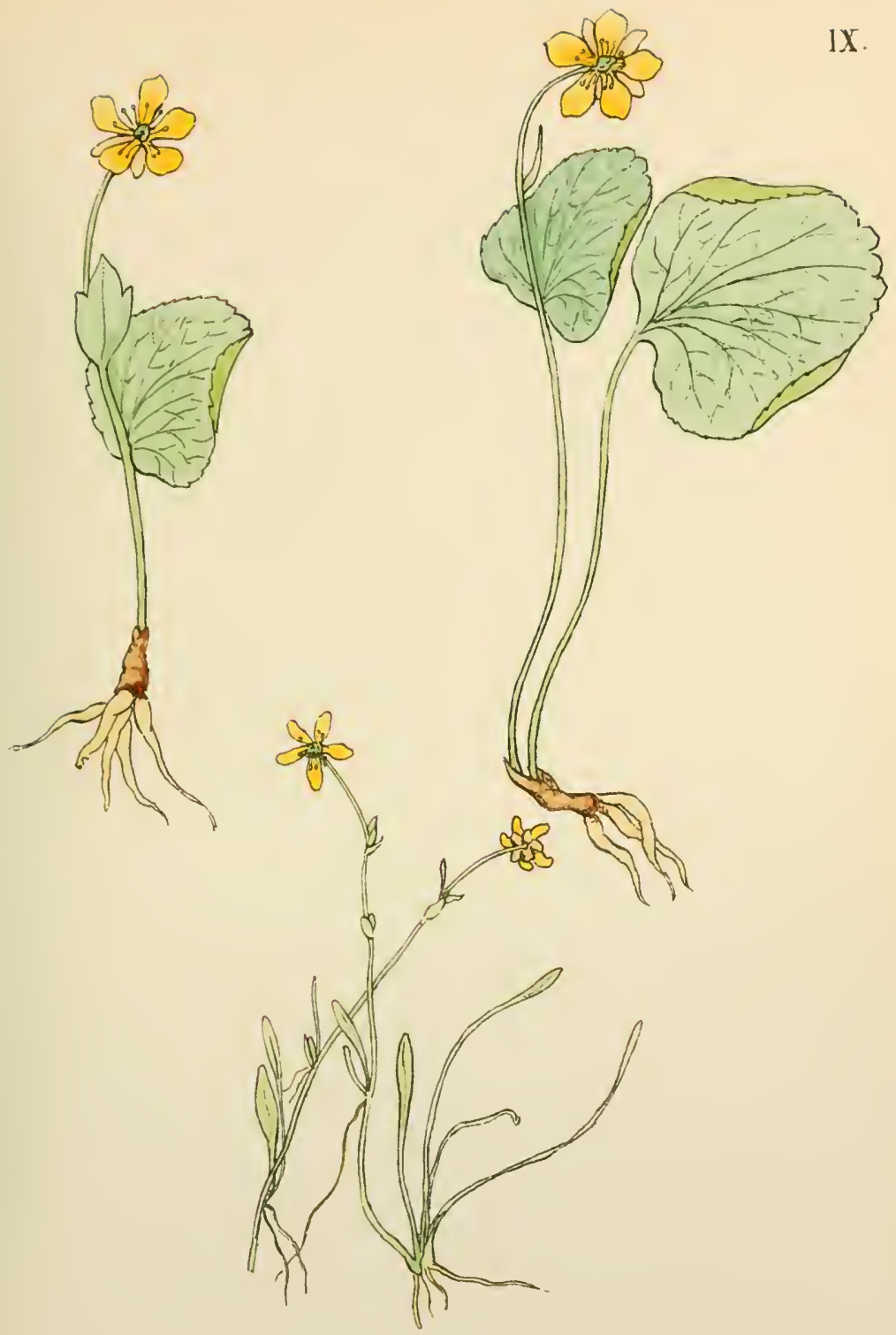

Ranunculus Thora.

Ranunculus hybridus.

Ranuneulus Flammula var. reptans. 

X.

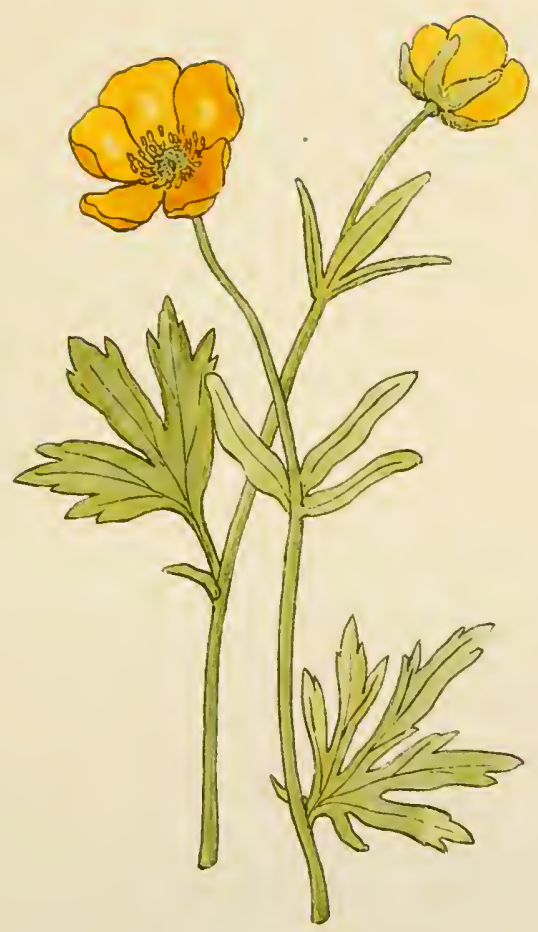

Aanunculus montanus. 

$X I$.

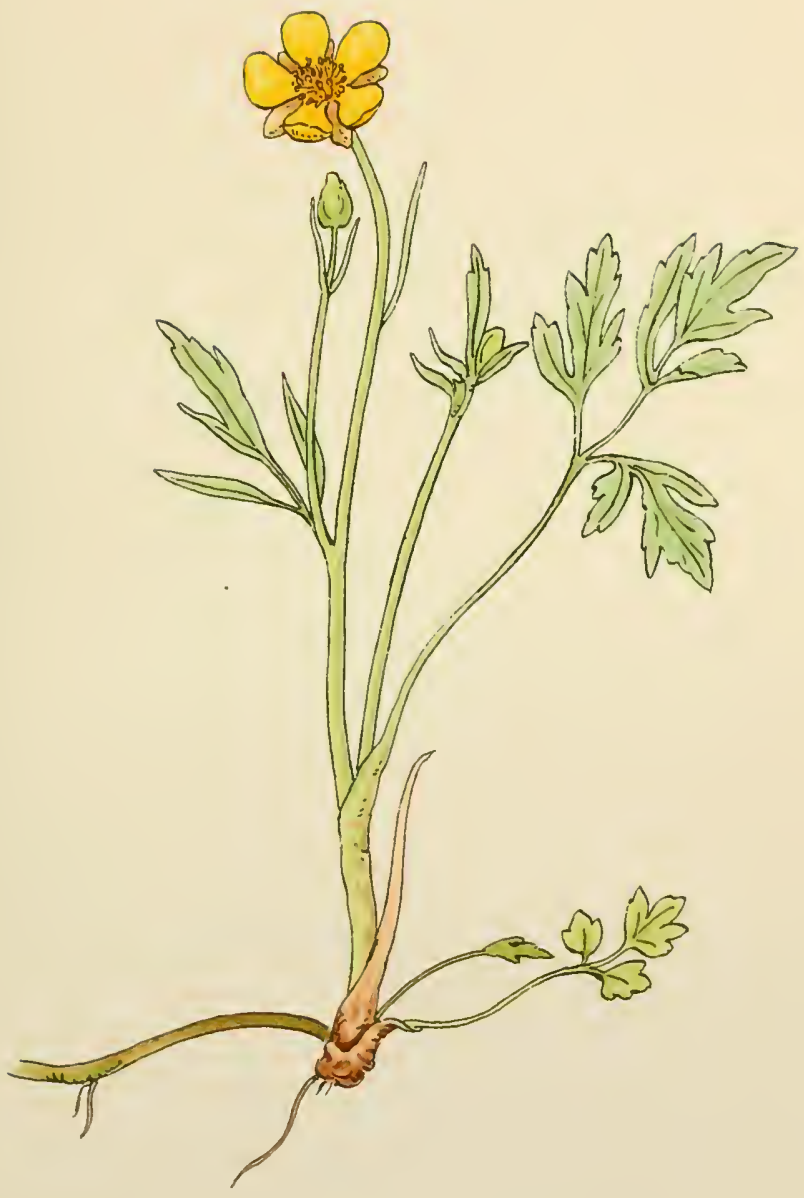

\footnotetext{
Ranunculus repens.
} 

XII.

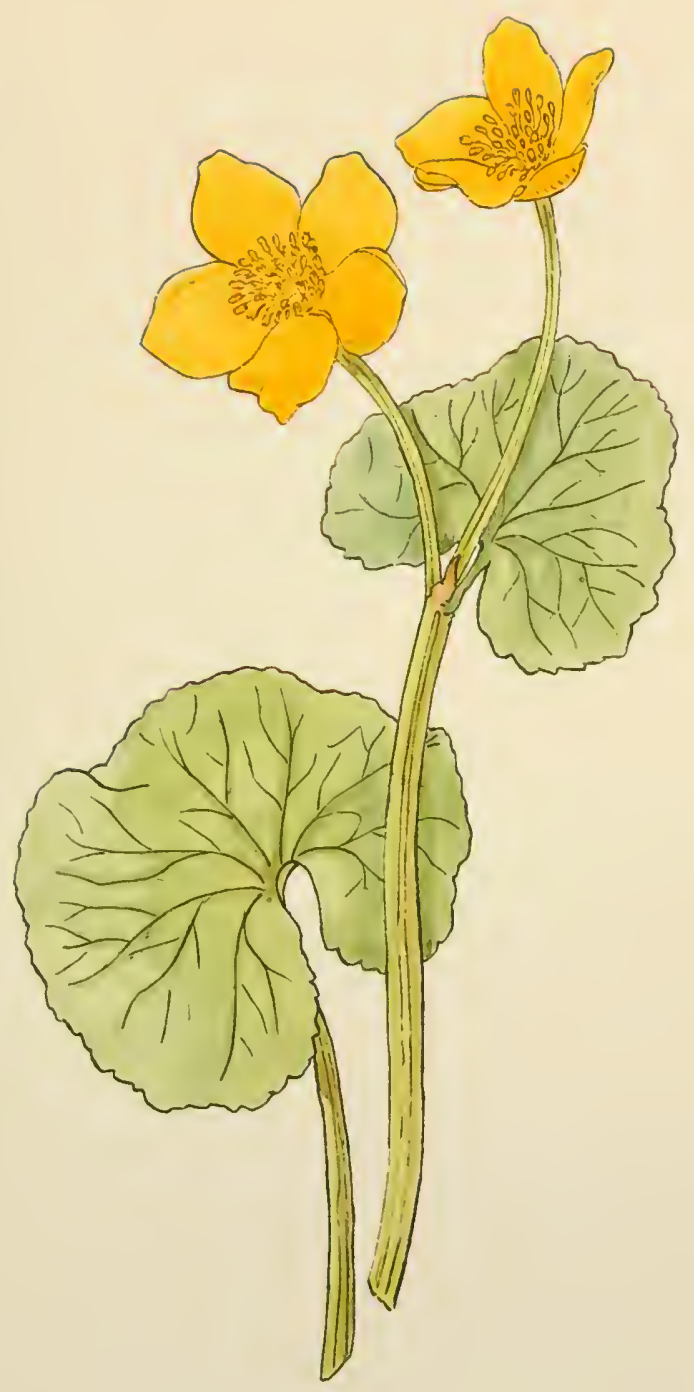

Caltha palustris. 
XIII.

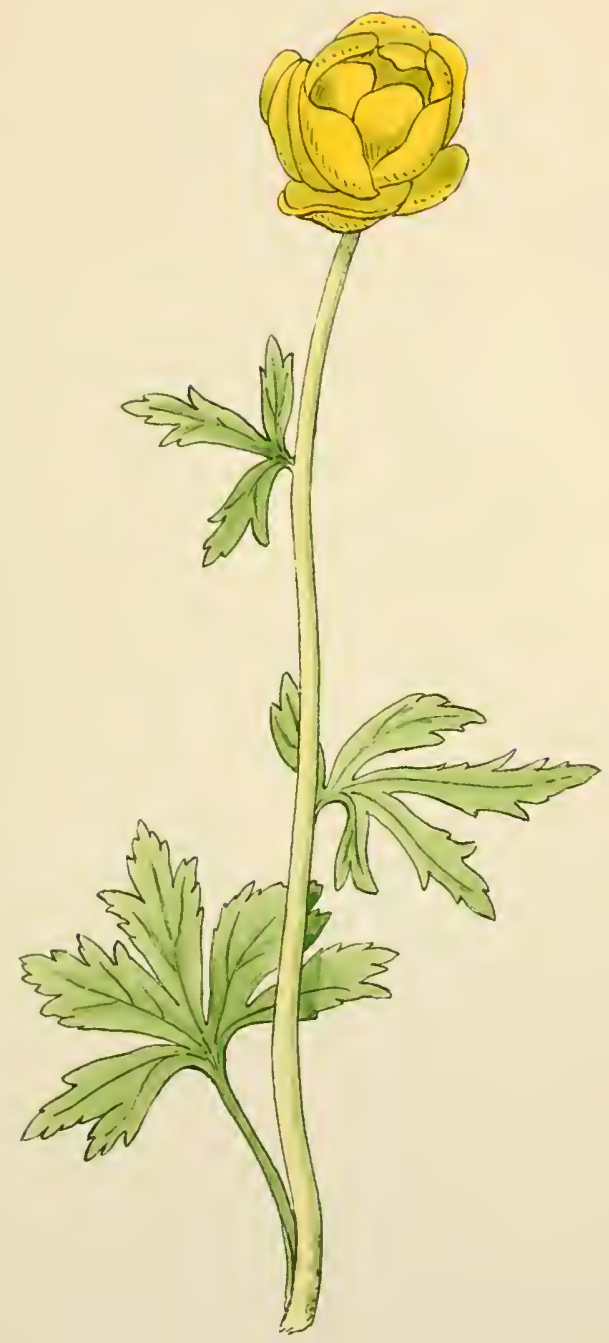

Trollius europæus. 

XIV.

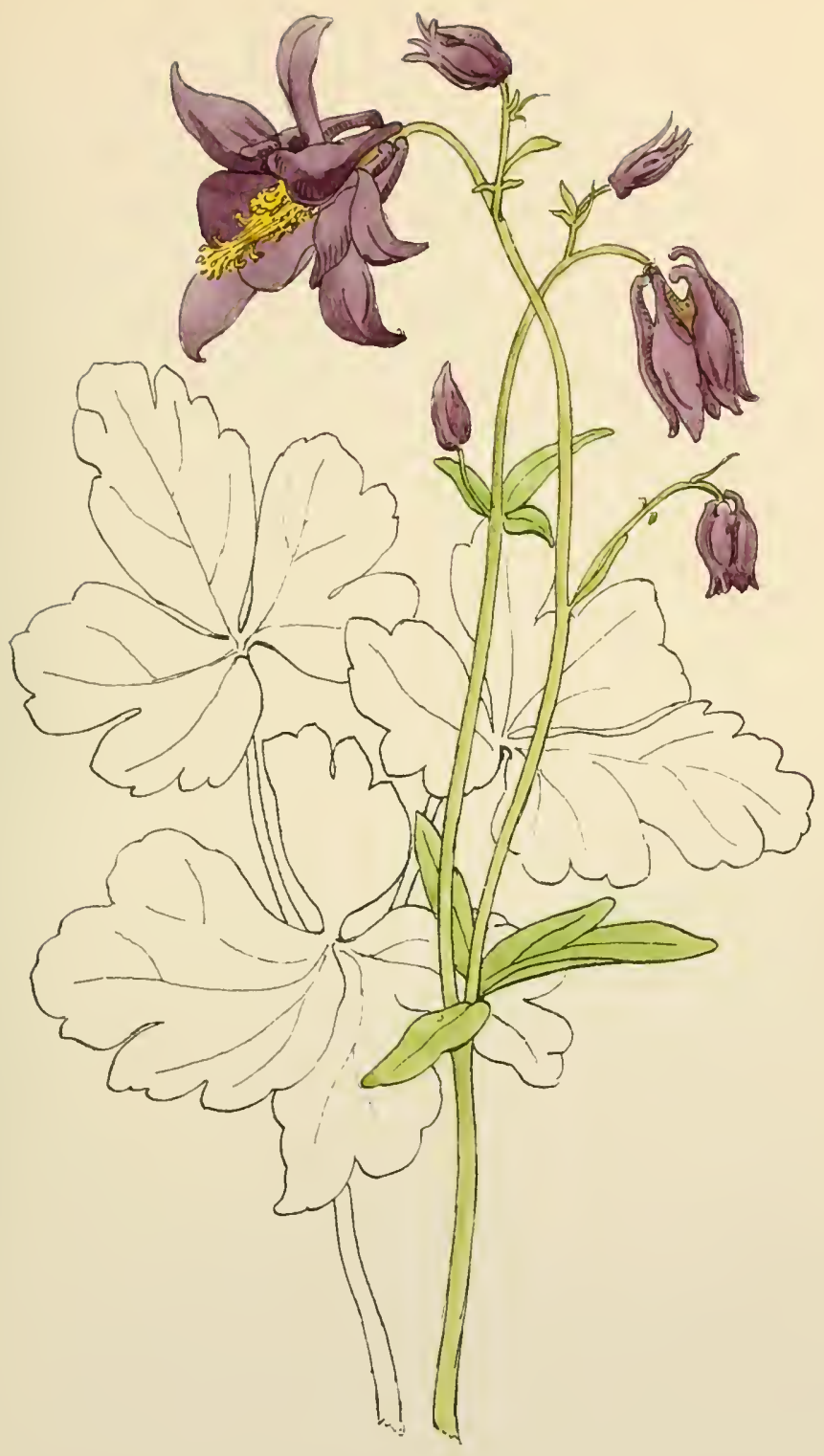

Aquilegia vulgaris, var. atrata. 


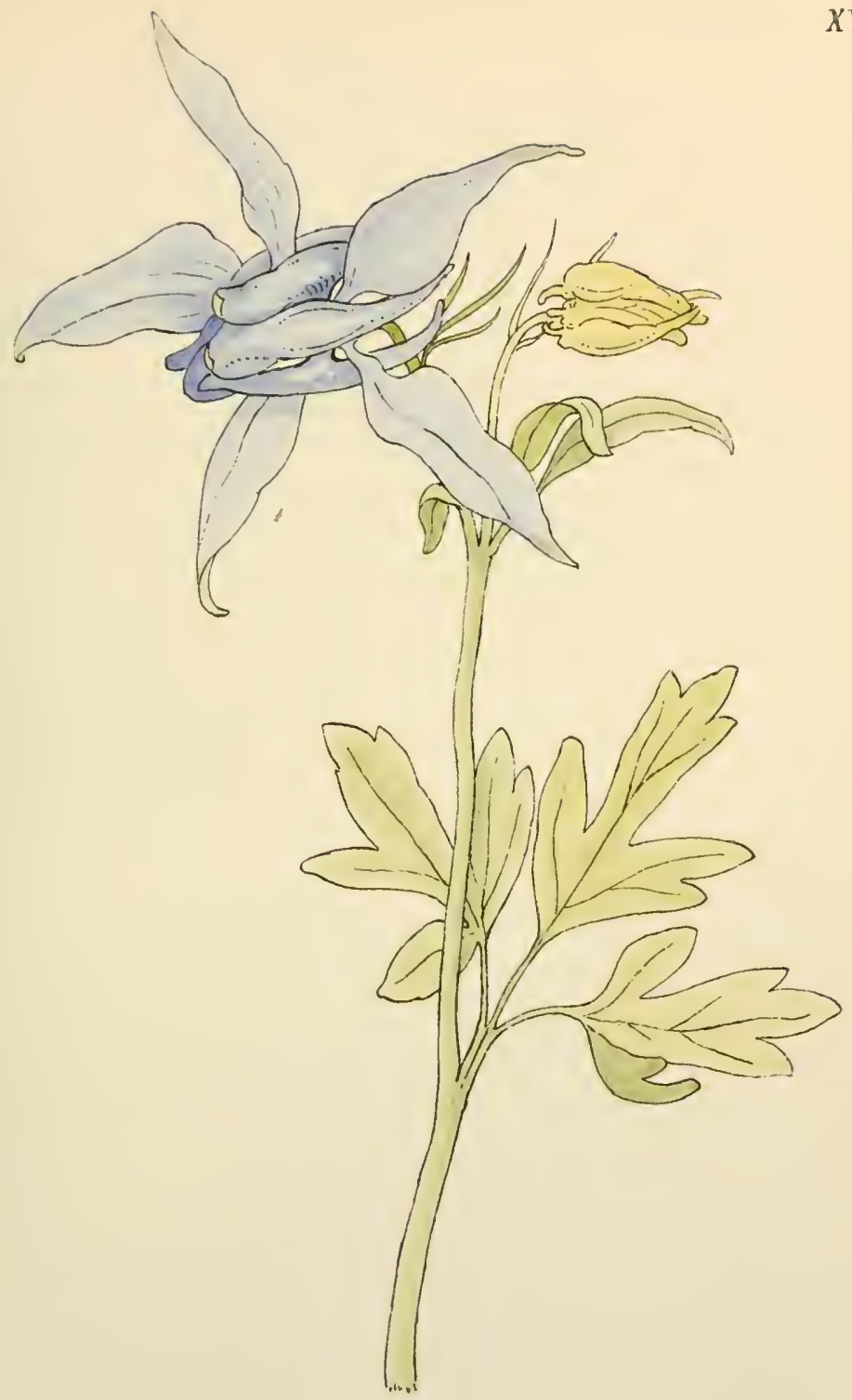

Aquilegia alpina. 


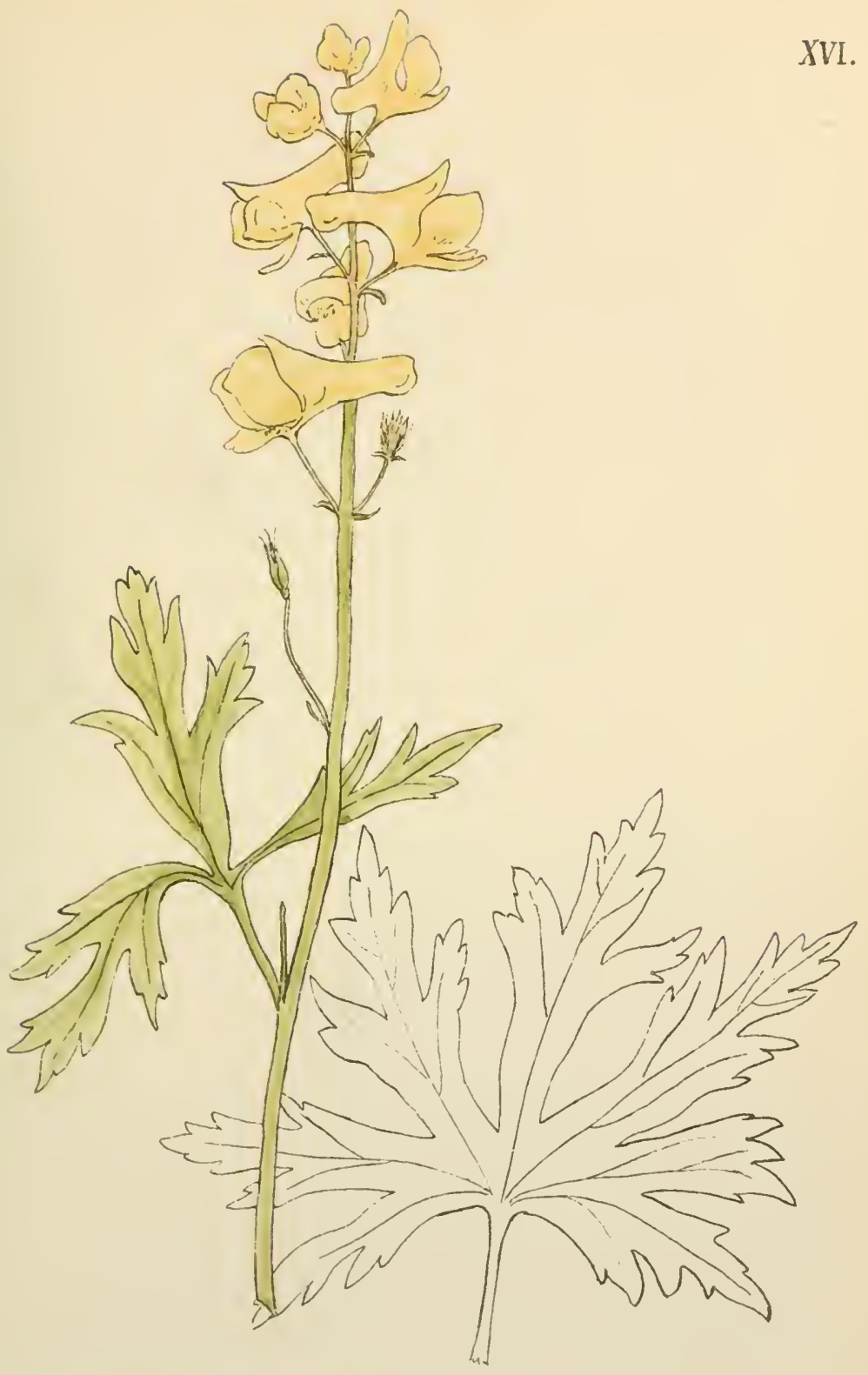

Aconitum lycoctonum. 


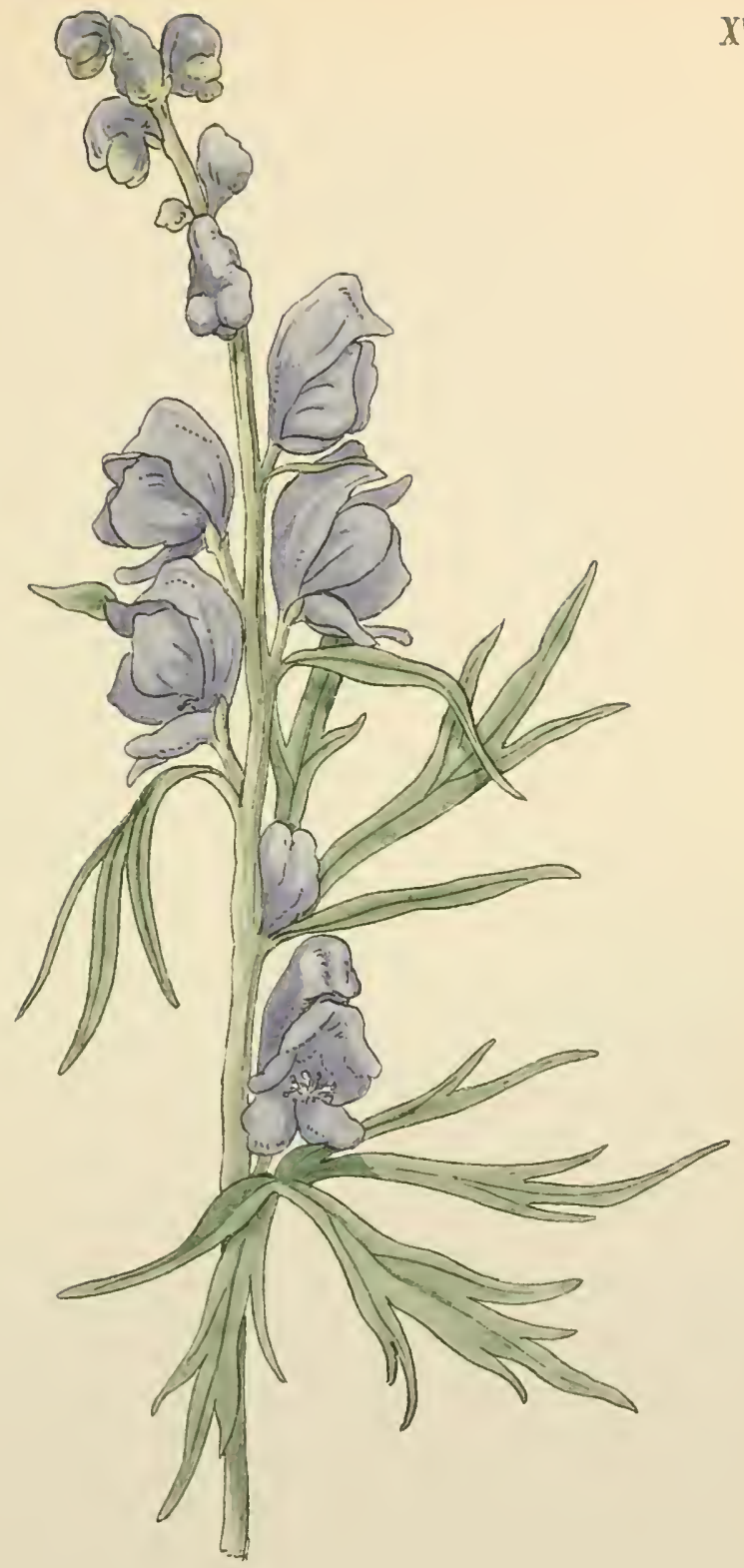

Aconitum Napellus. 

XVIIl.

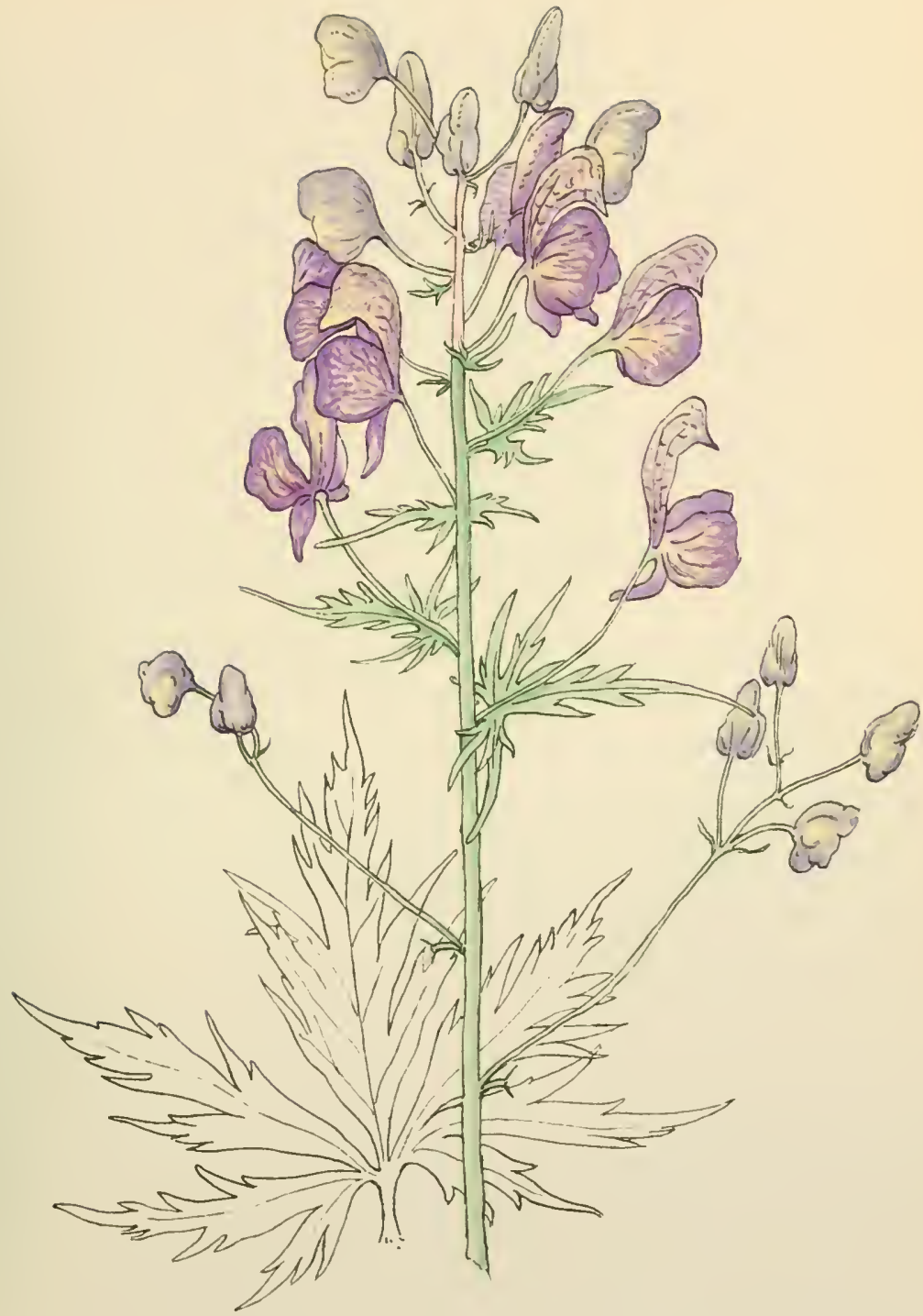

Aconitum paniculatum. 


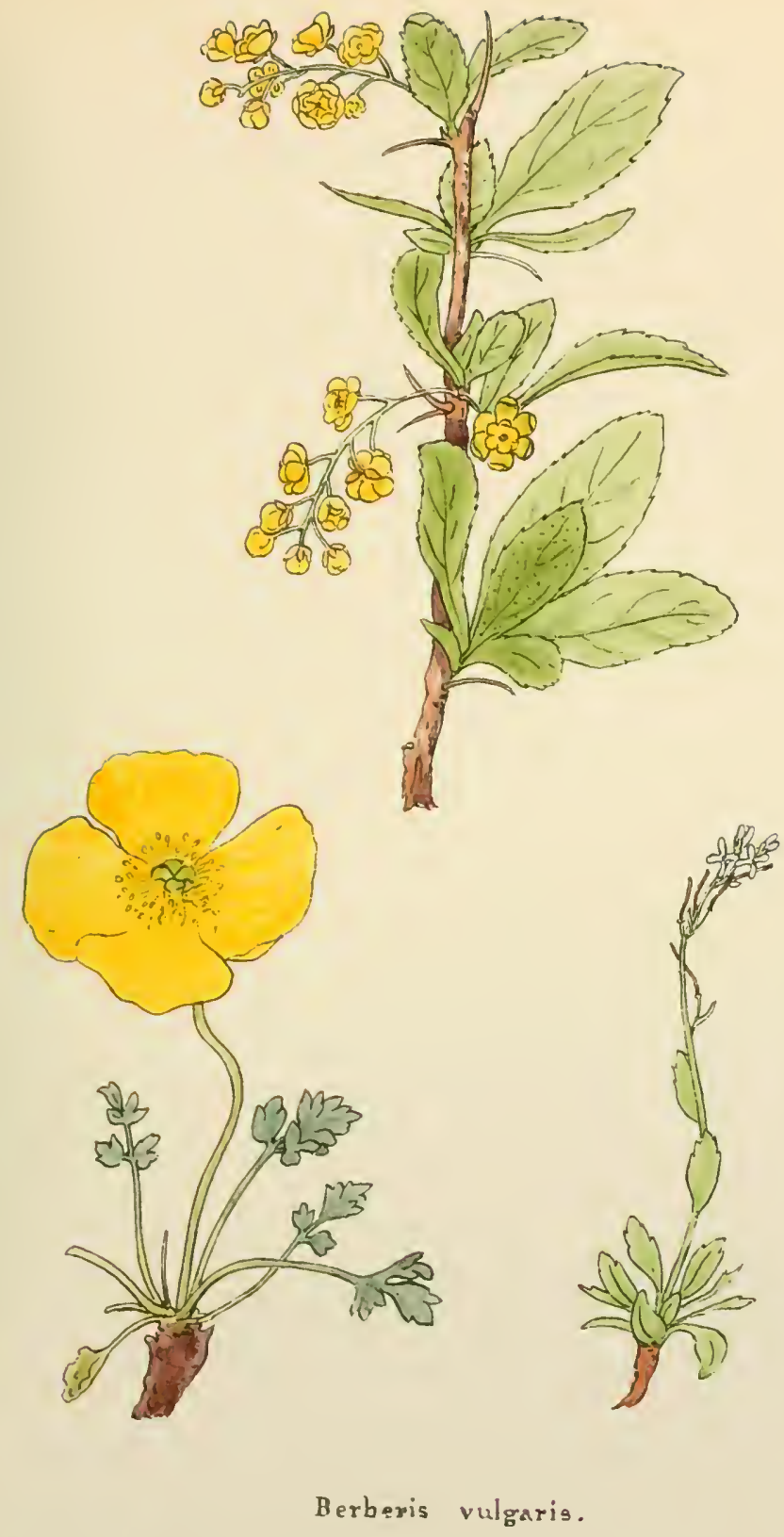

Papaver alpinum var. flaviflorum.

Arabis cærulea. 


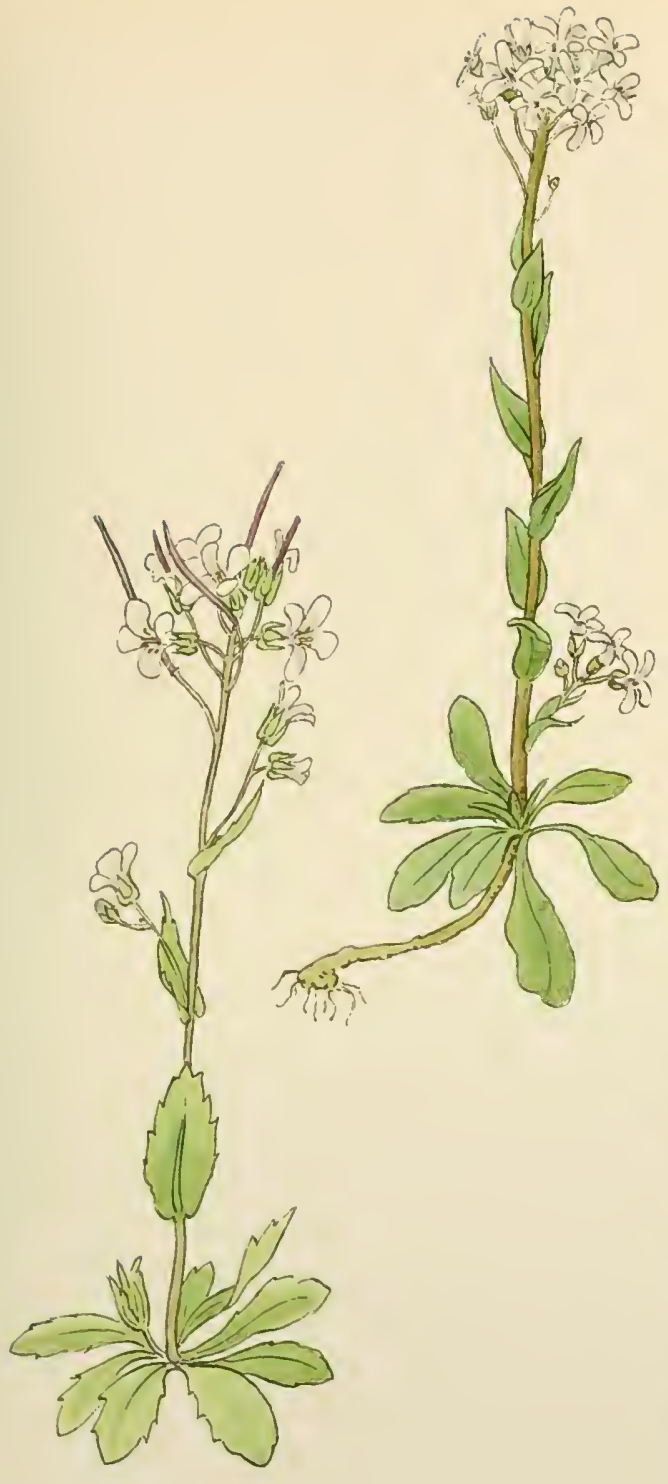

$X X$.

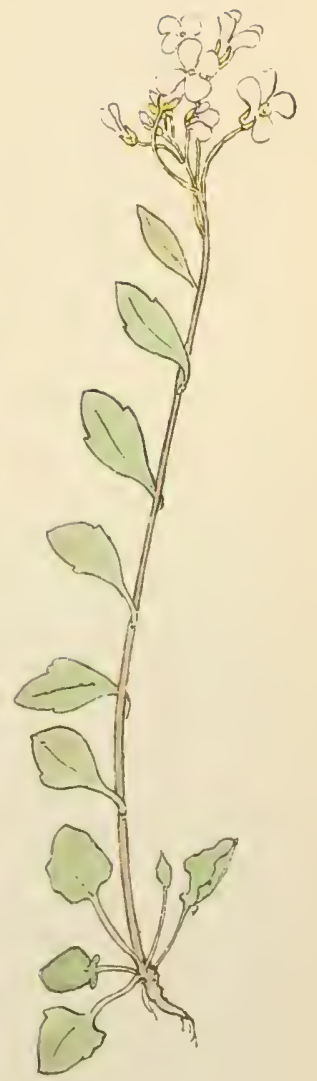

Arabis bellidifolia.

Arabis alpins.

Arabis Halleri. 


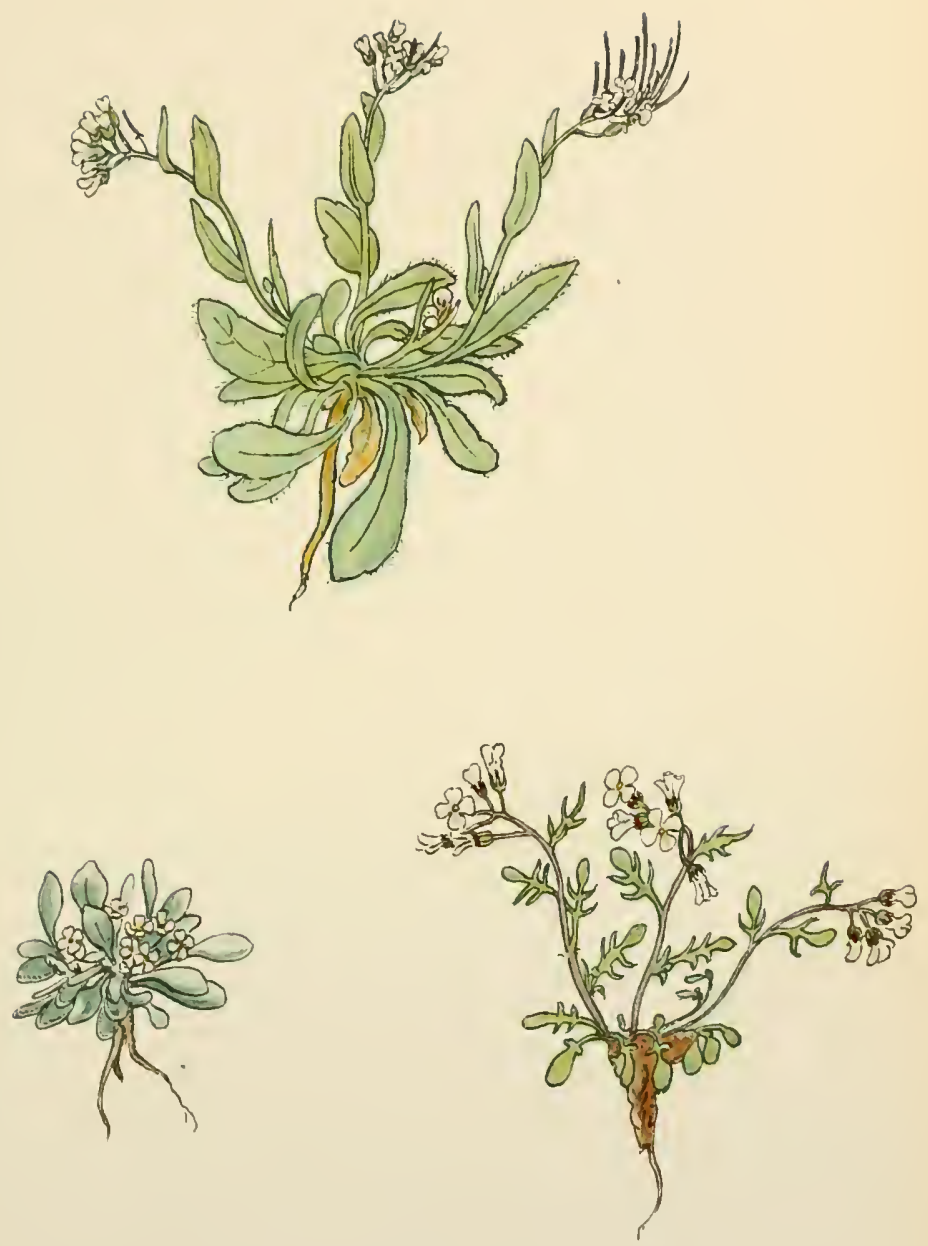

Arabie alpestris var. ciliata.

Caxdamine alpina. Cardamine resedefolia. 

XXII.
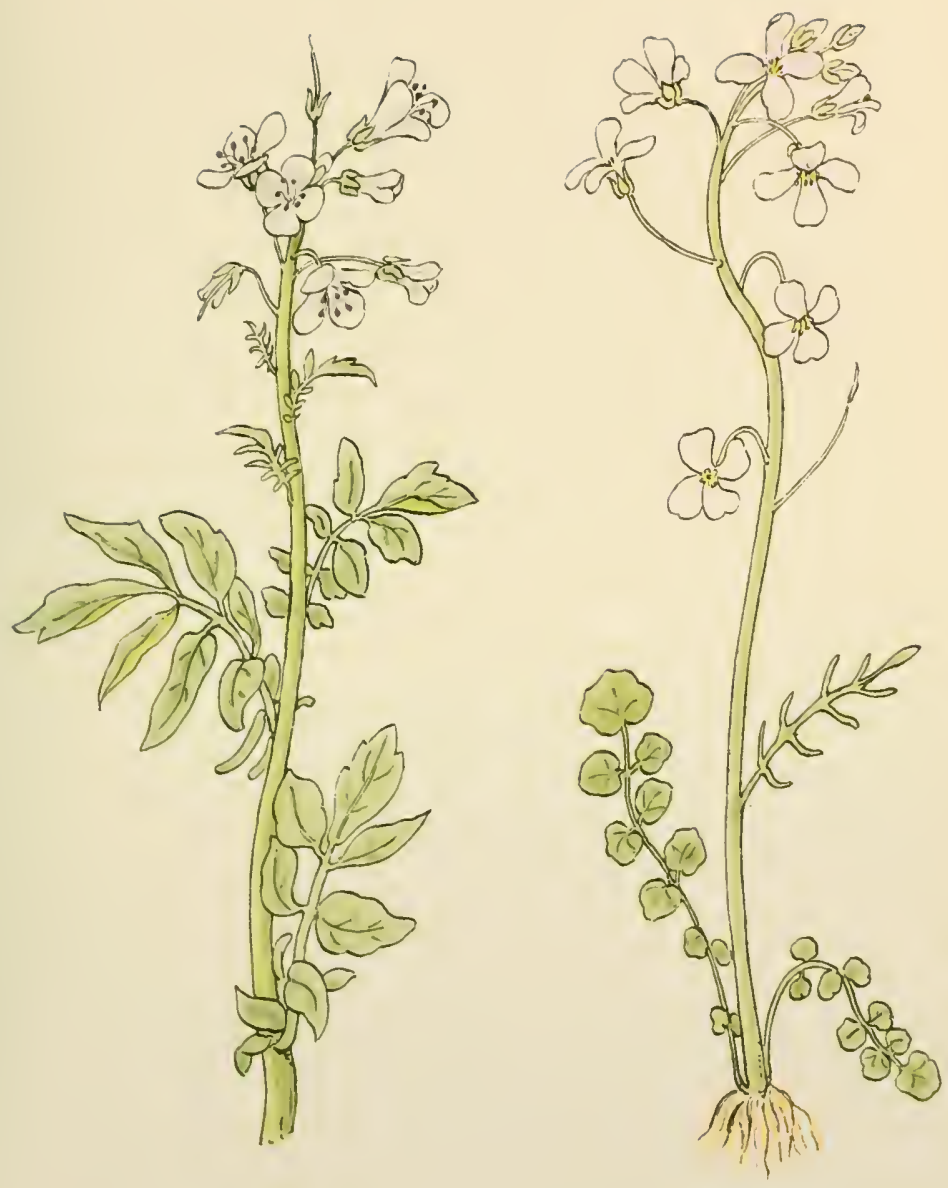

Cardamine amara.

Cardamine pratensis. 

XXIII.

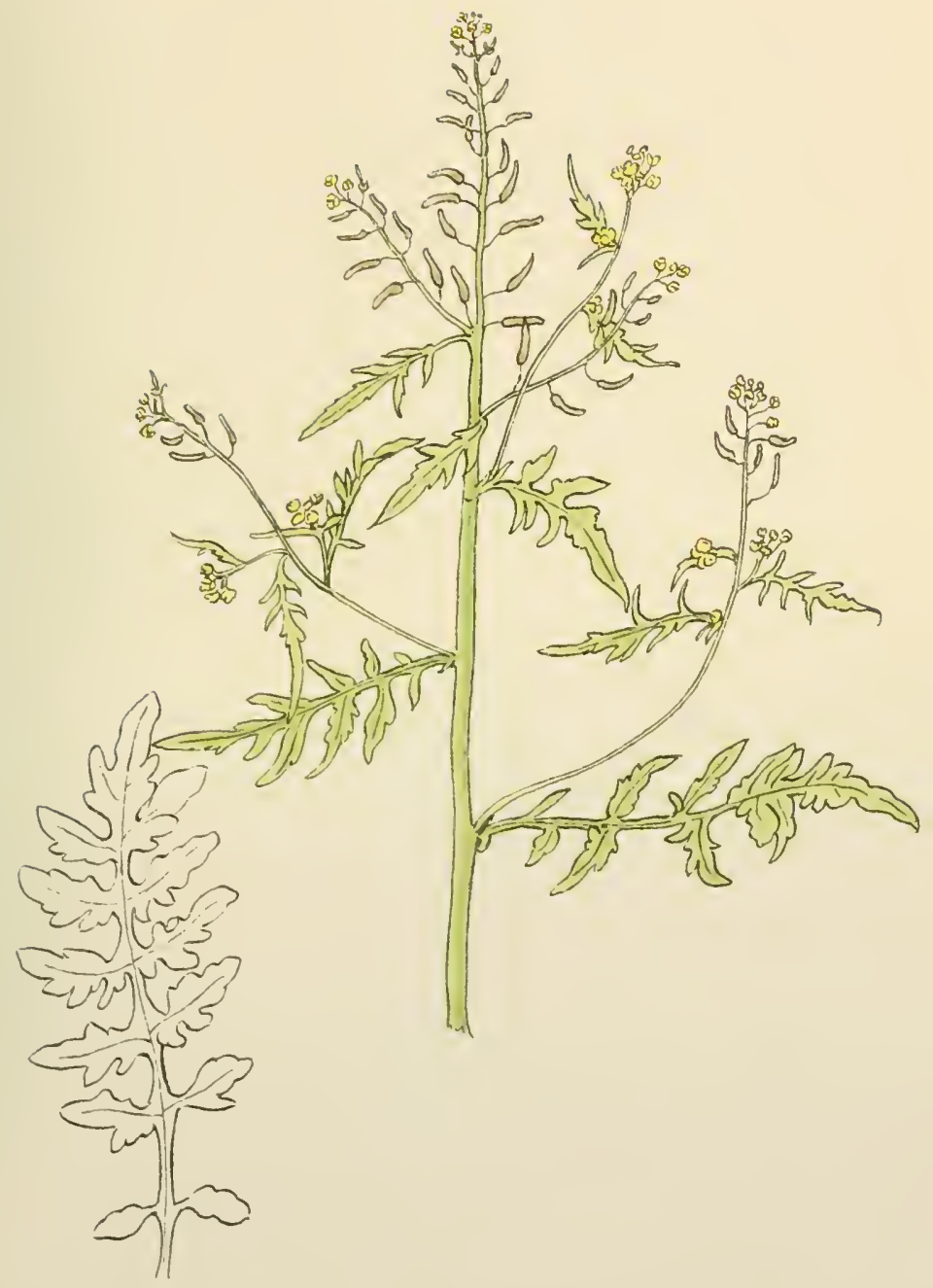

Nasturtium palustre. 
XXIY.

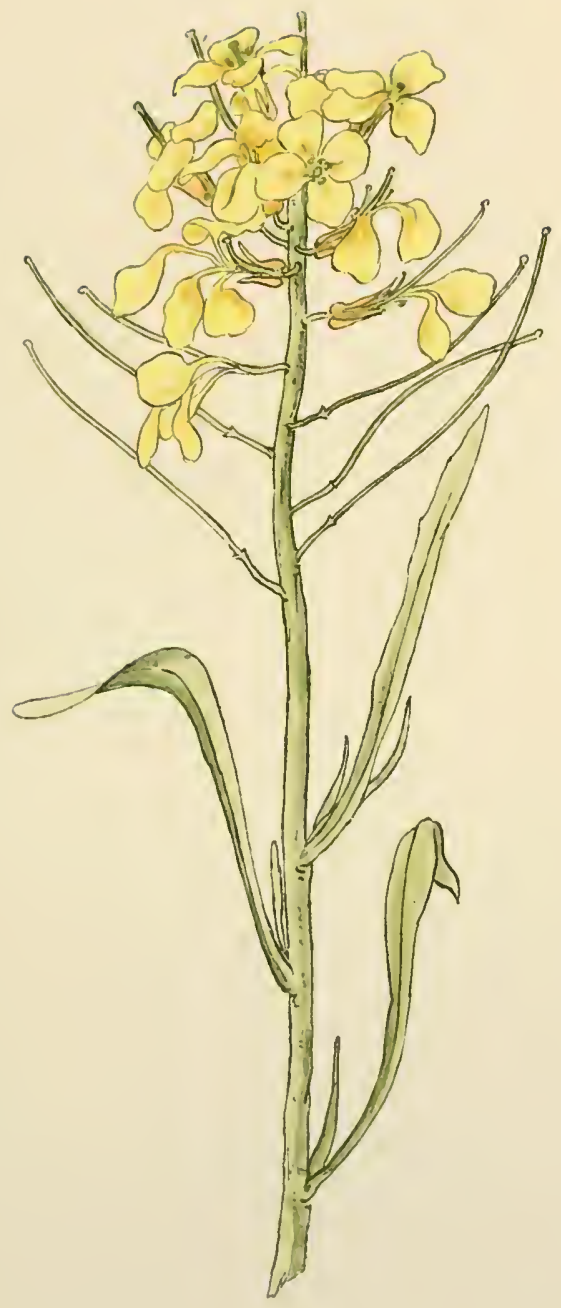

Erysinum rhaticum. 



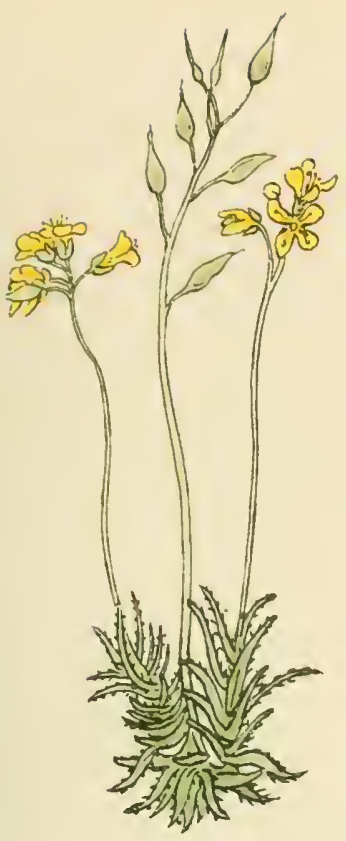

XXV.
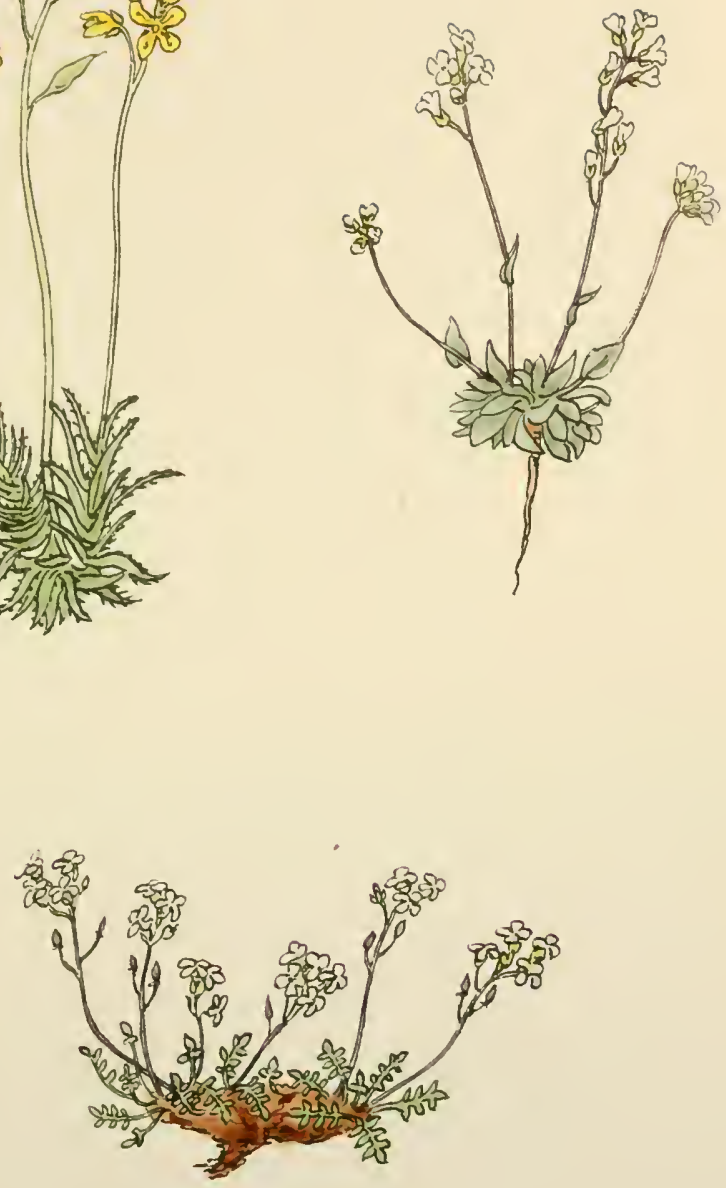

Draba aizoïdes.

Draba tomentosa var. frigida.

Hutchinsia alpina. 


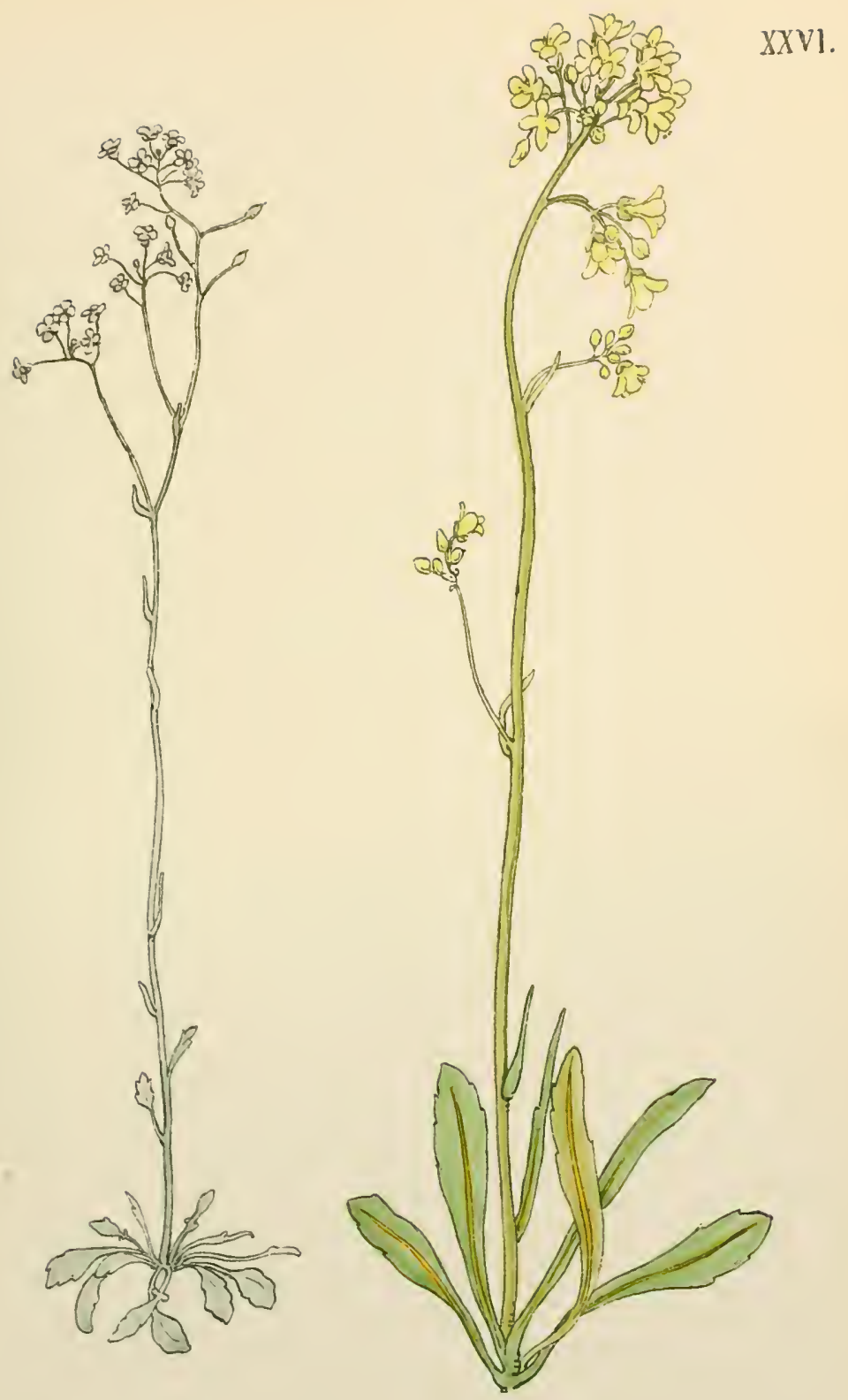

Cochlearia saxatilis. Biscutella levigata. 


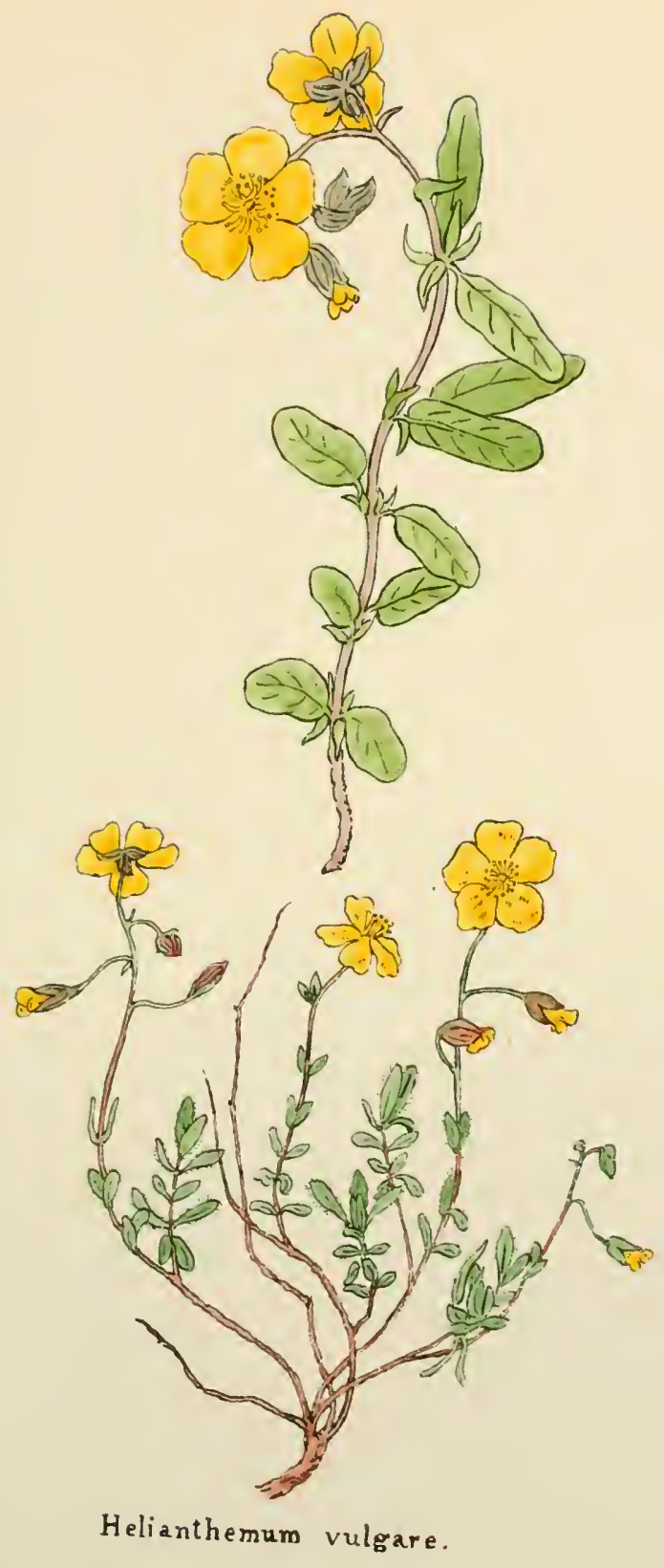

XXVII.

Helianthemum CElandicuur. 



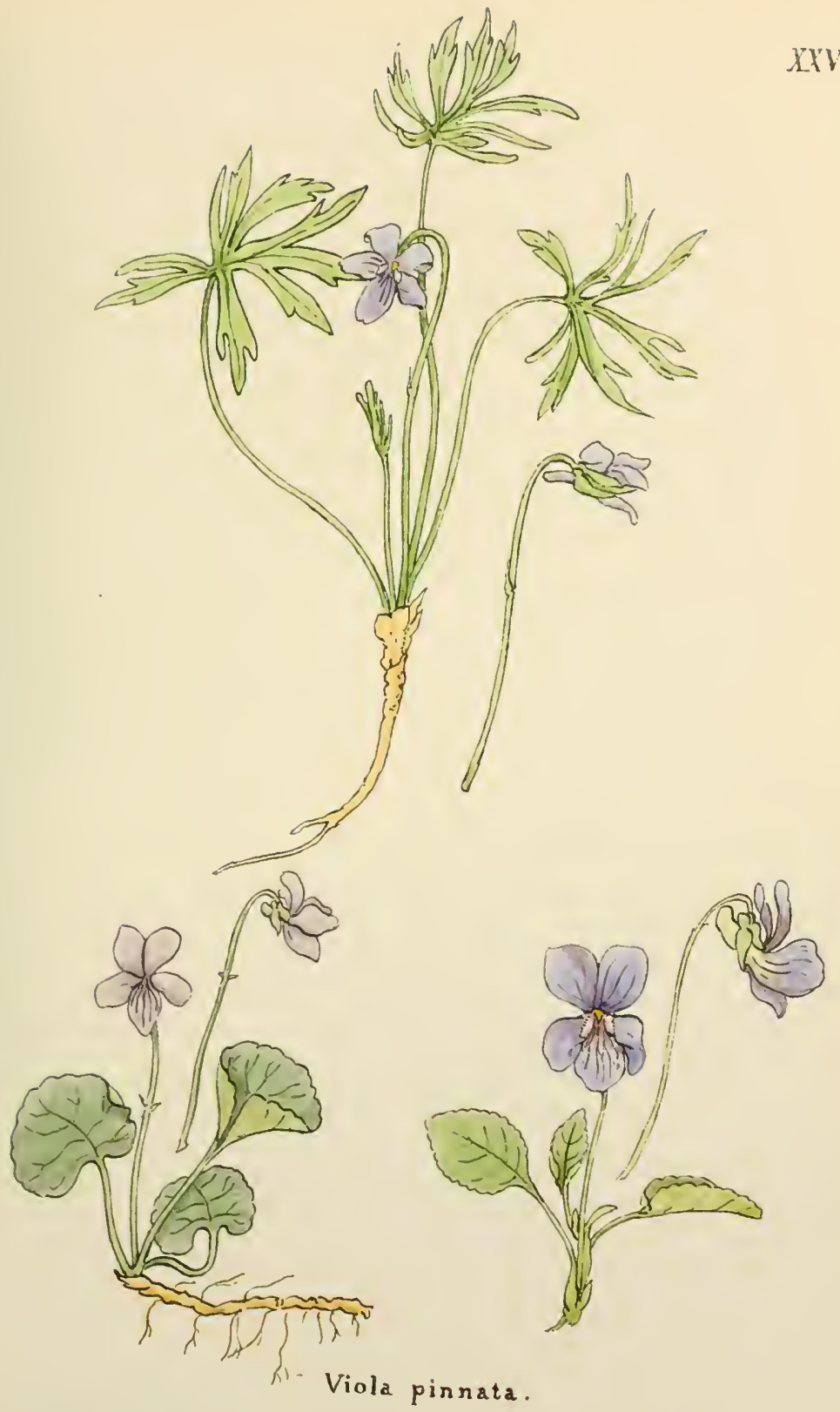

Viola palustris.

Viola canins. 

XXIX .

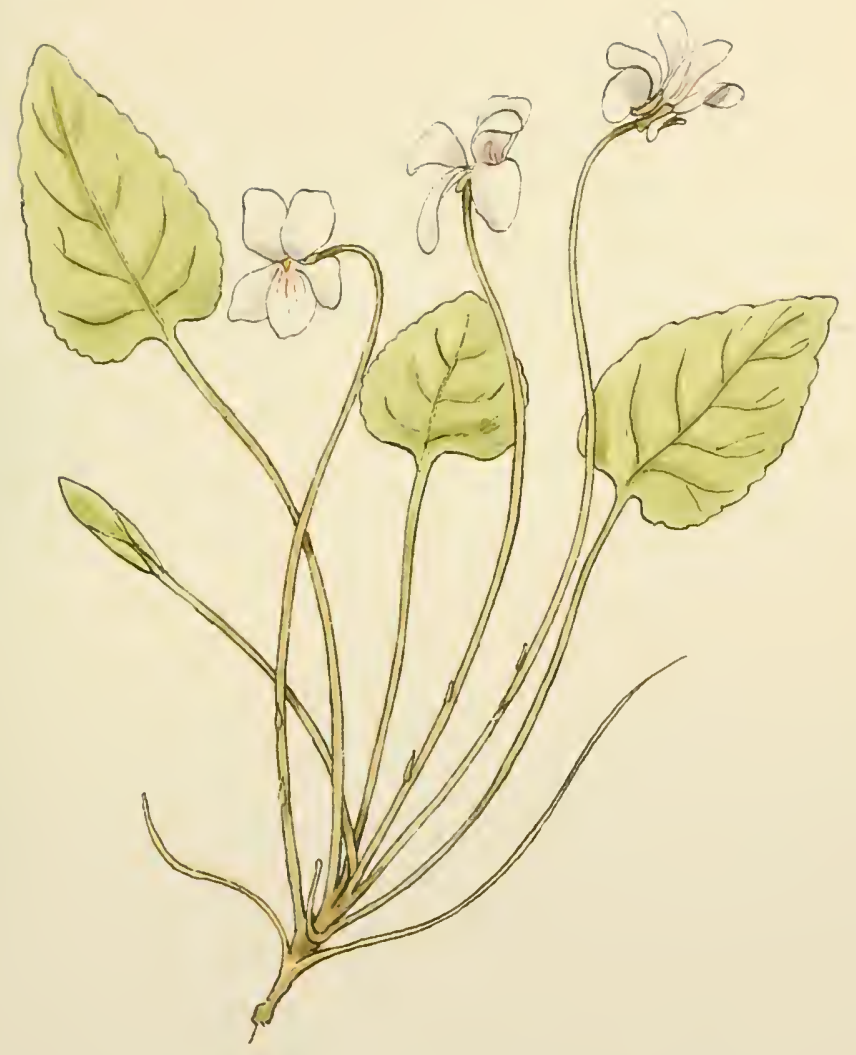

Viola Thomasiana. 
XXX.

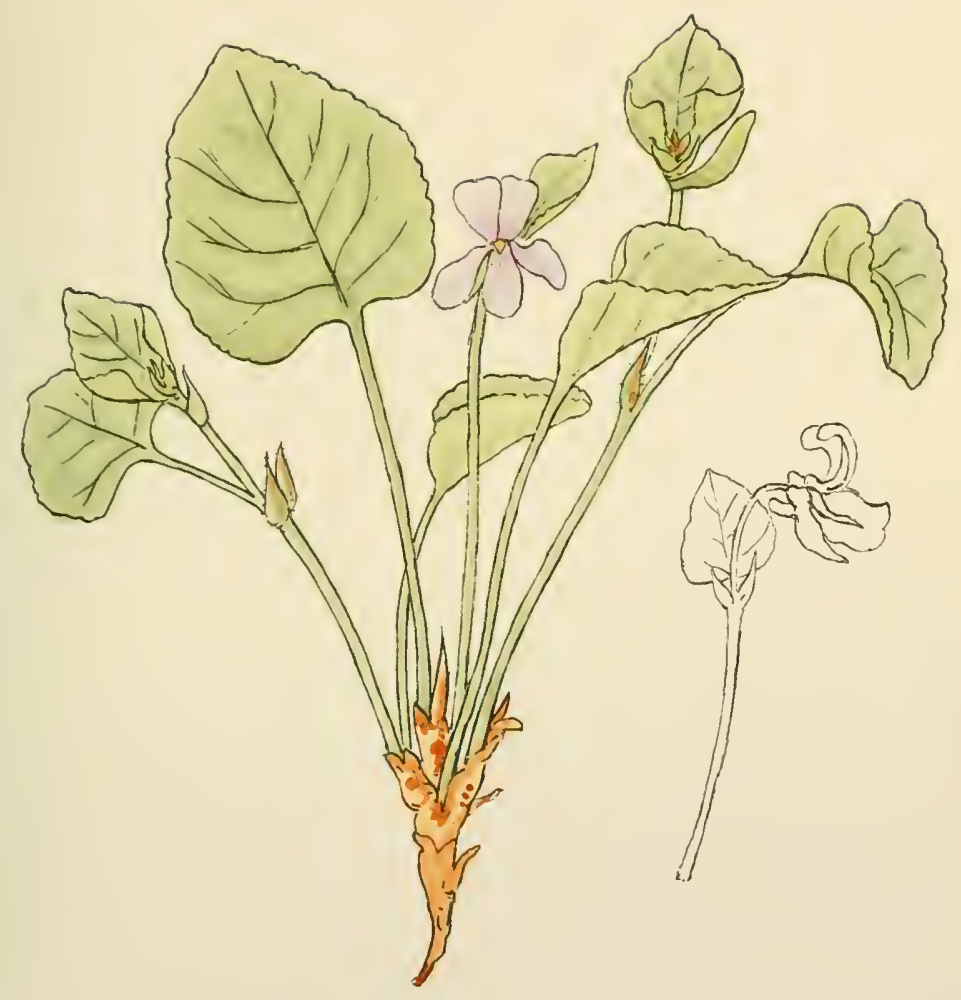

Viola mirabilis. 


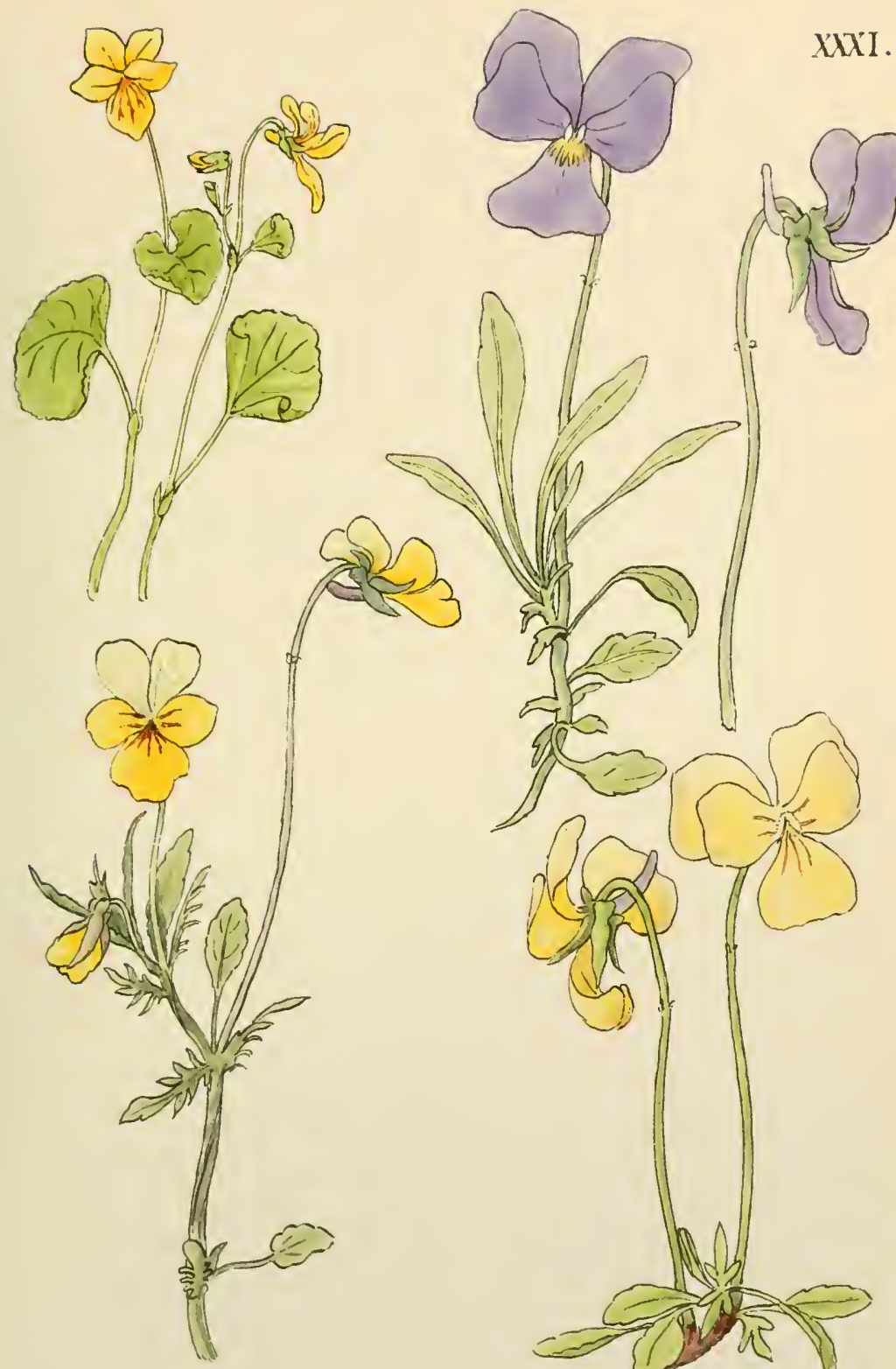

Viola biflora.

Viola tricolor.

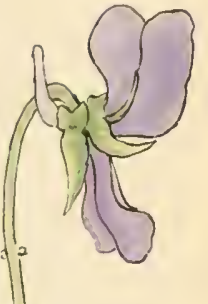





\section{XXXI1.}

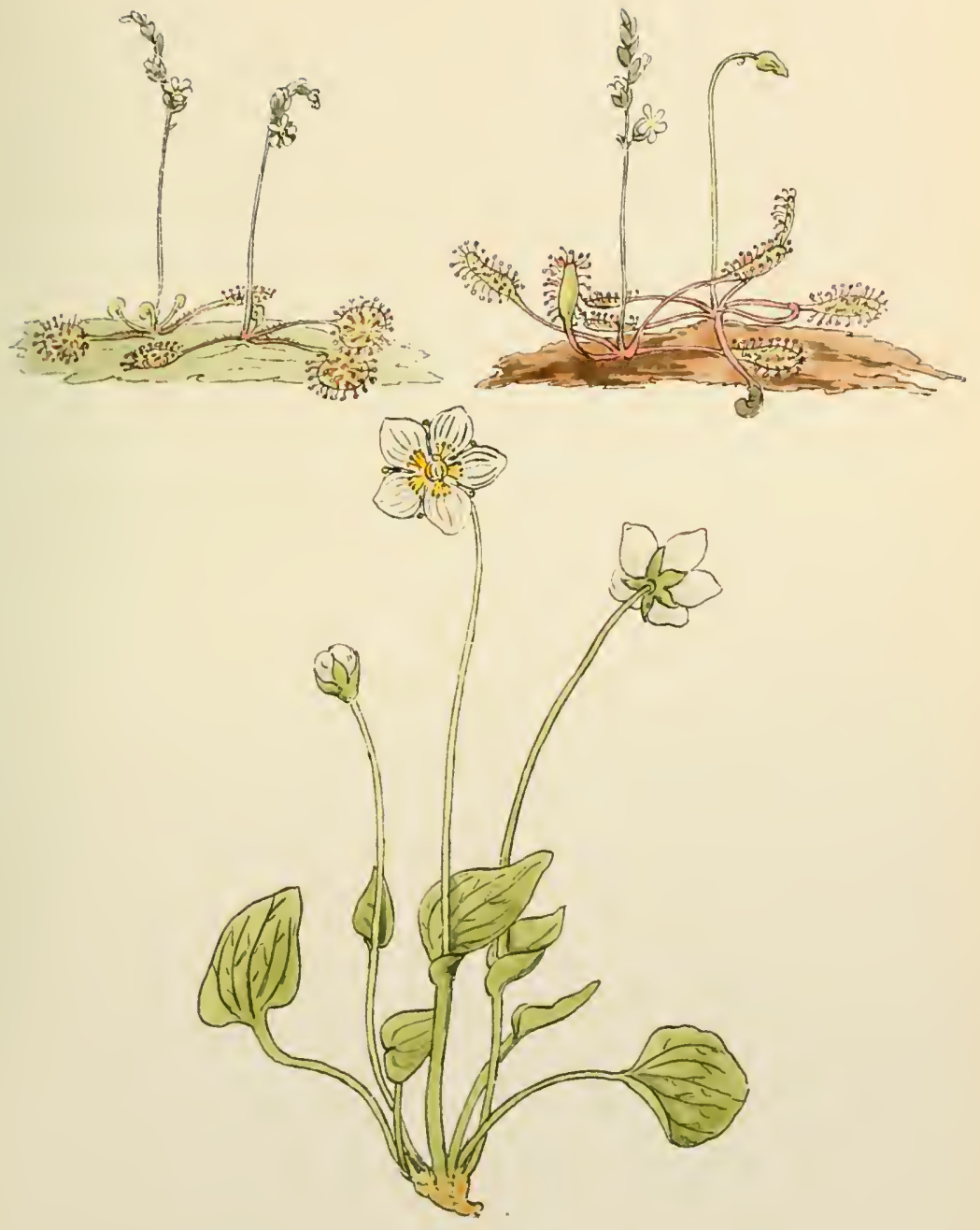

Drosera rotundifolia.

Drosera lungifolia.

Parnassia palustris. 

XXXIII.

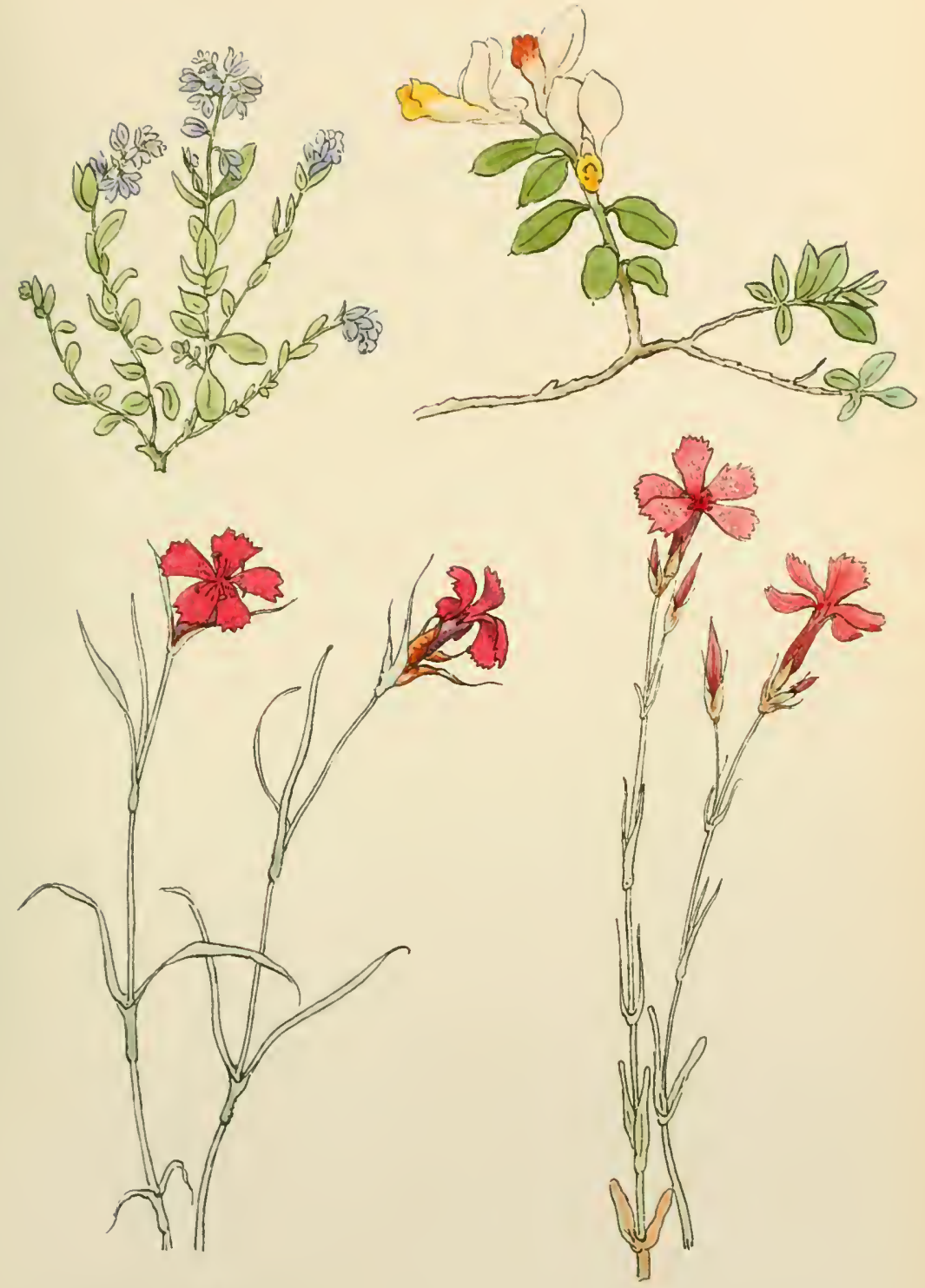

Polygala amara.

Polygala Chamabuxus.

Dianthus atro-rubens.

Dianthus deltoídes. 
XXXIV.
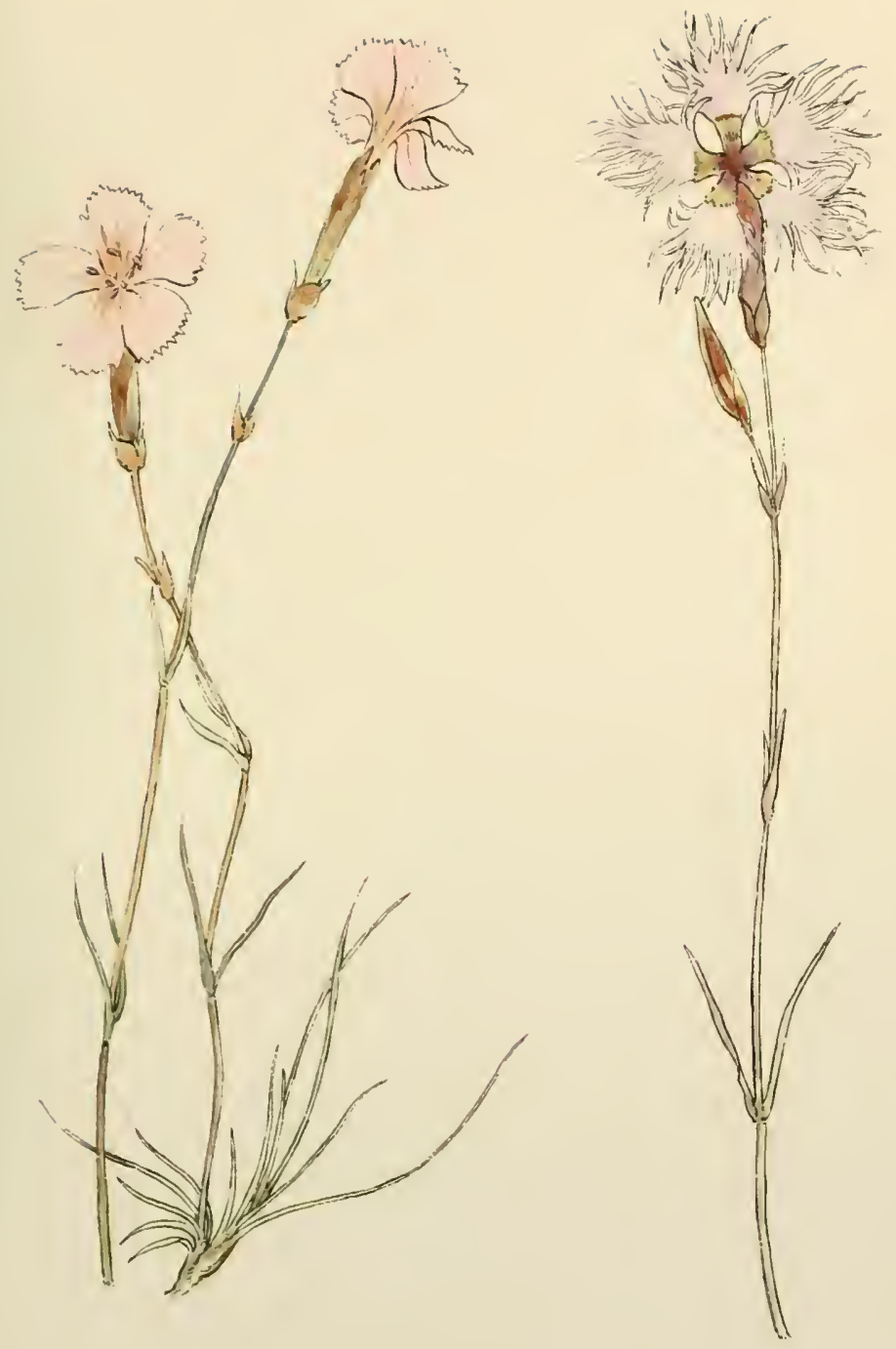

Dianthus sylvestris.

Dianthus superbus. 



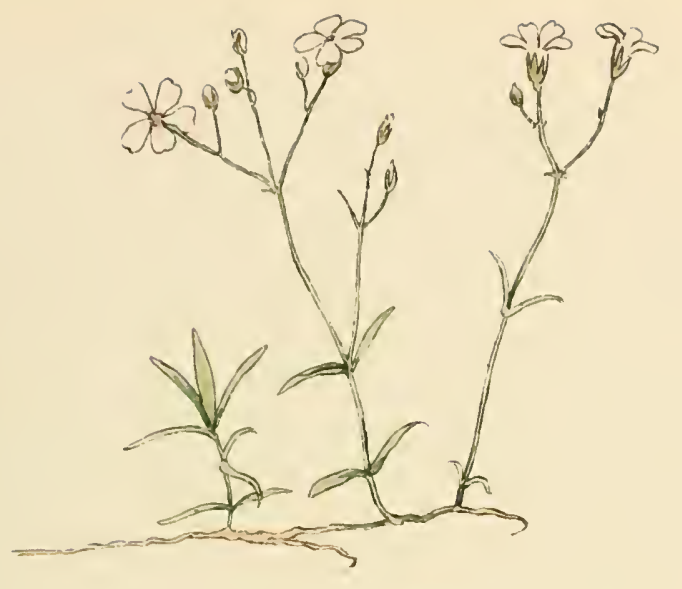

XXXV.

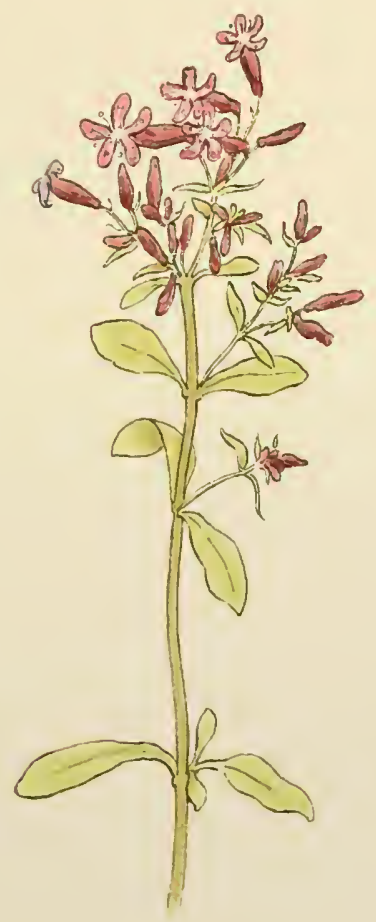

Gypsophila repens.

Seponaria ocymuïdes. 

XXXVI.
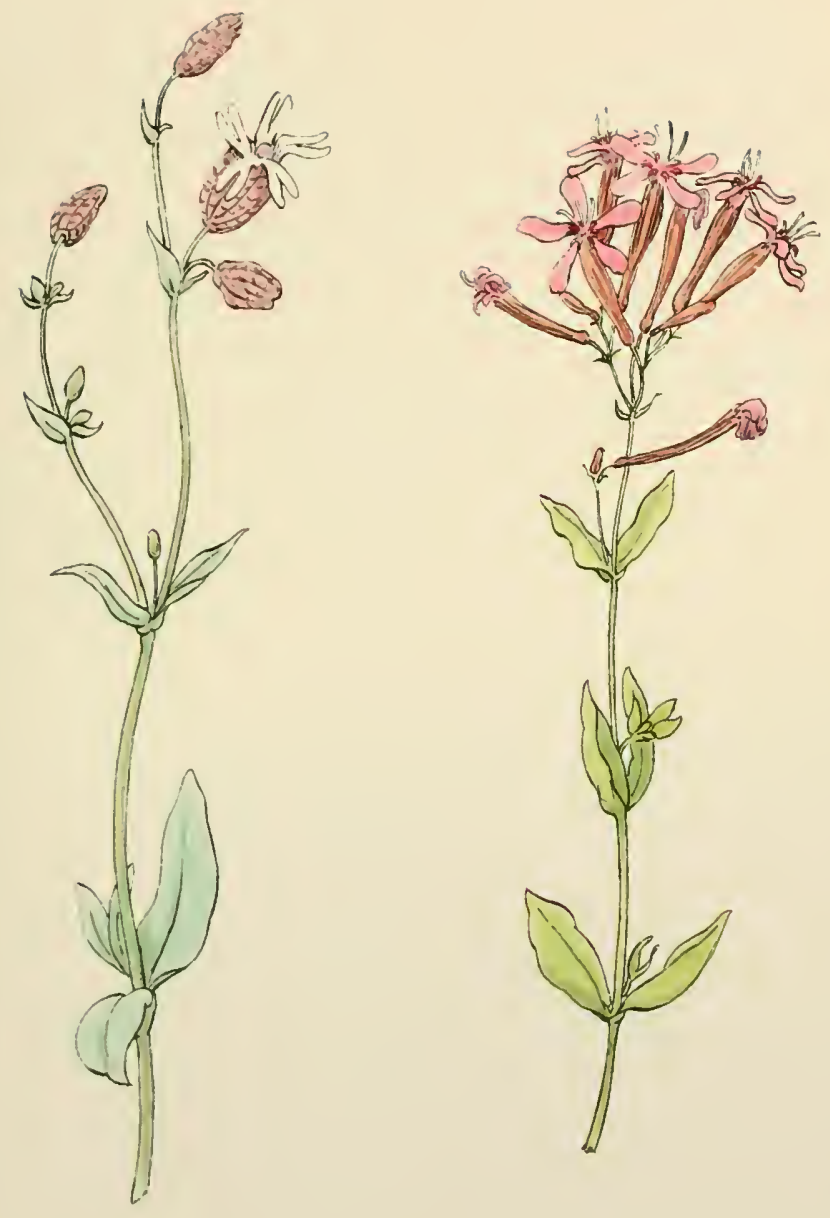

Silene inflata.

Silene Armeria. 


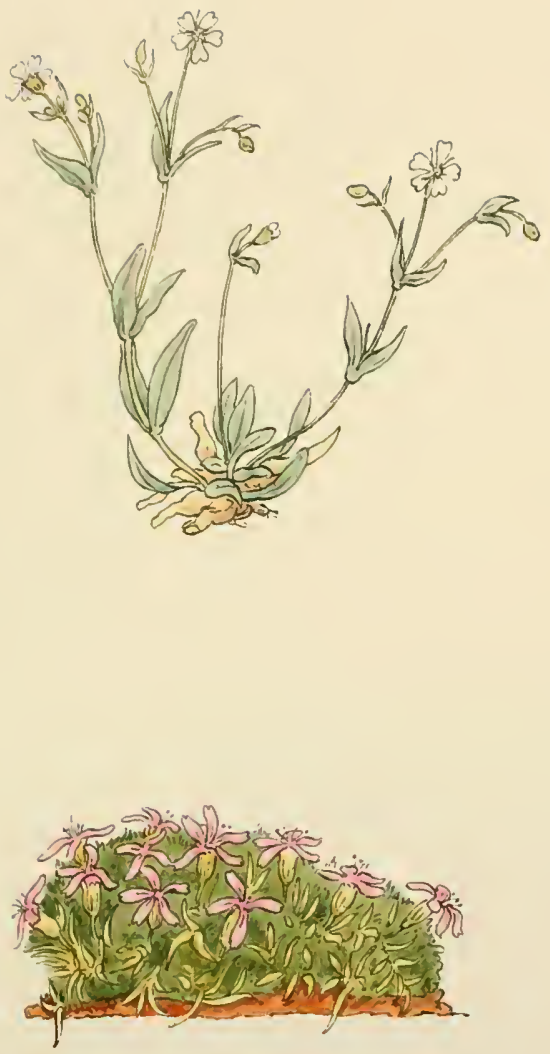

Silene rupegtxis.

Silene acaulis. 



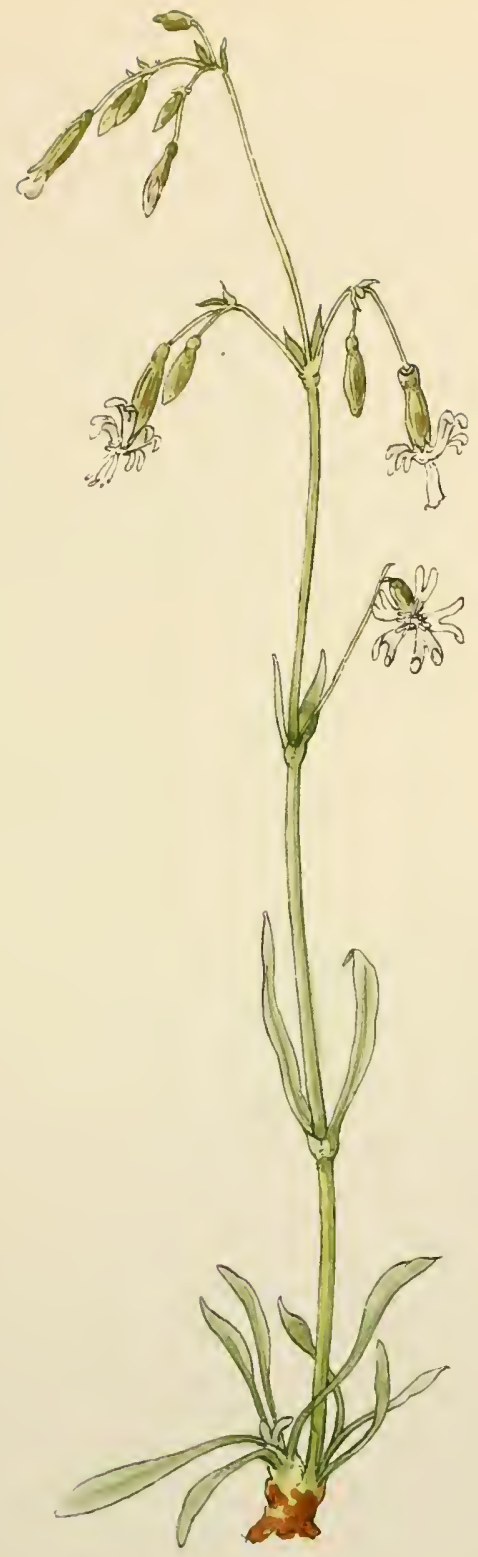

XXXVIII.

Silene nutans. 

XXXIX .

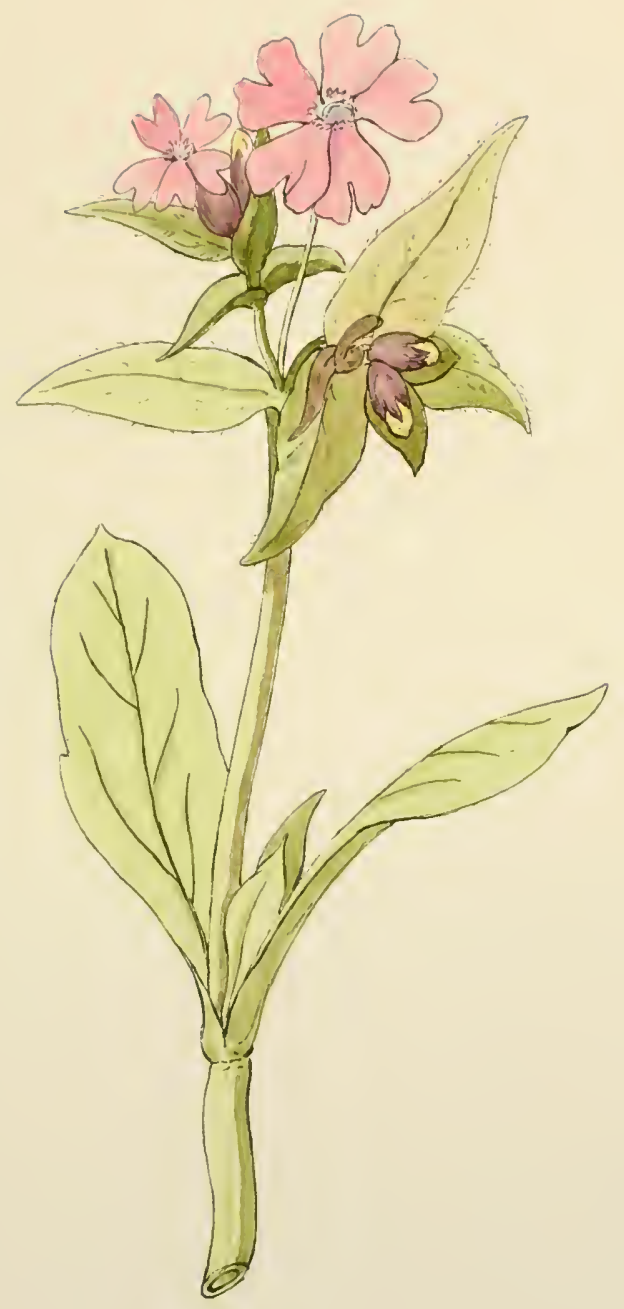

Melandrium sylvestre. 

$X \mathrm{~L}$.
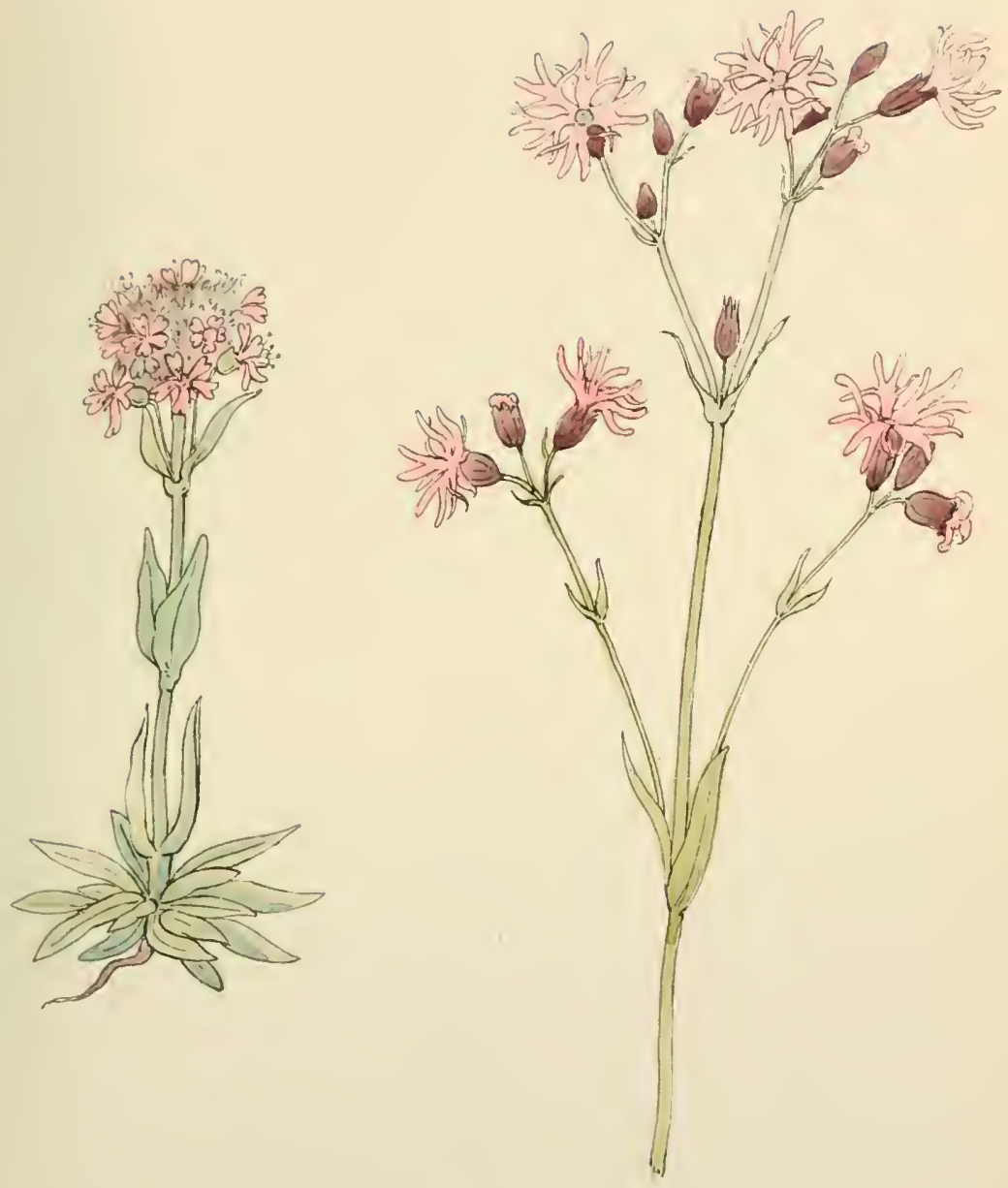

Lychnis alpina.

Lychnis Flos-ruculi. 


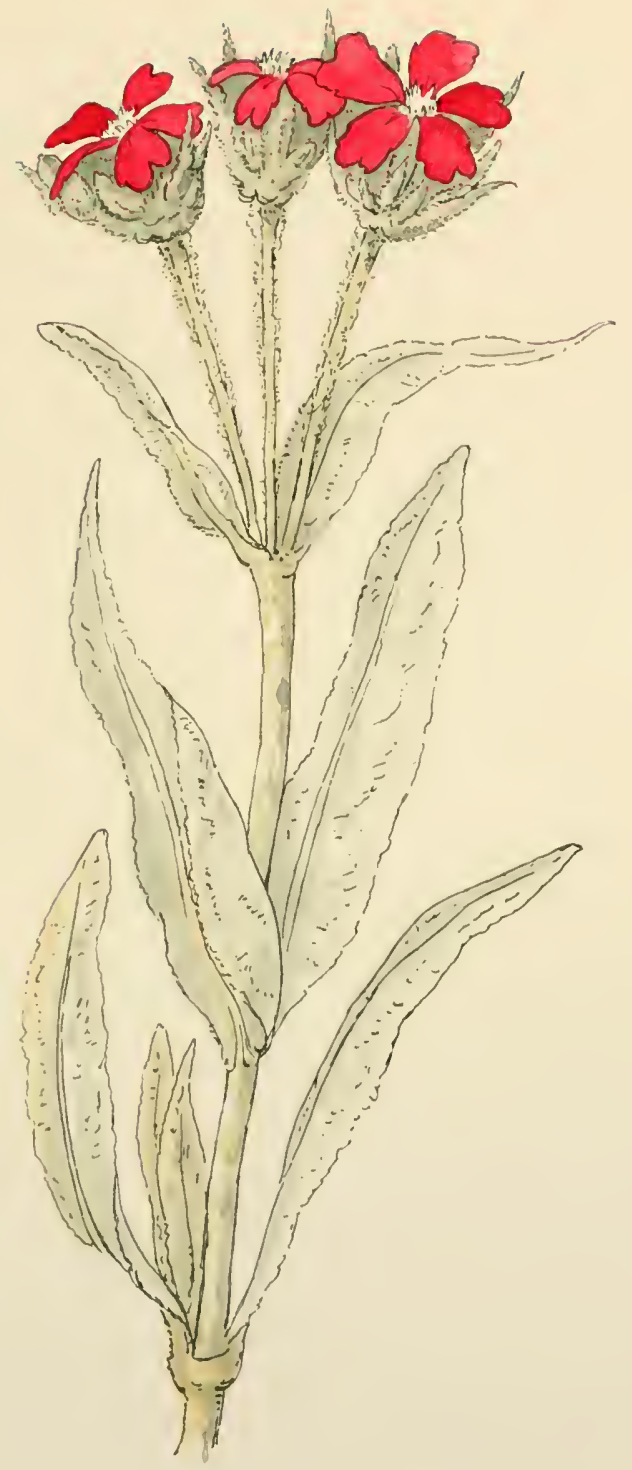

Lychnis Flos-Jovis. 

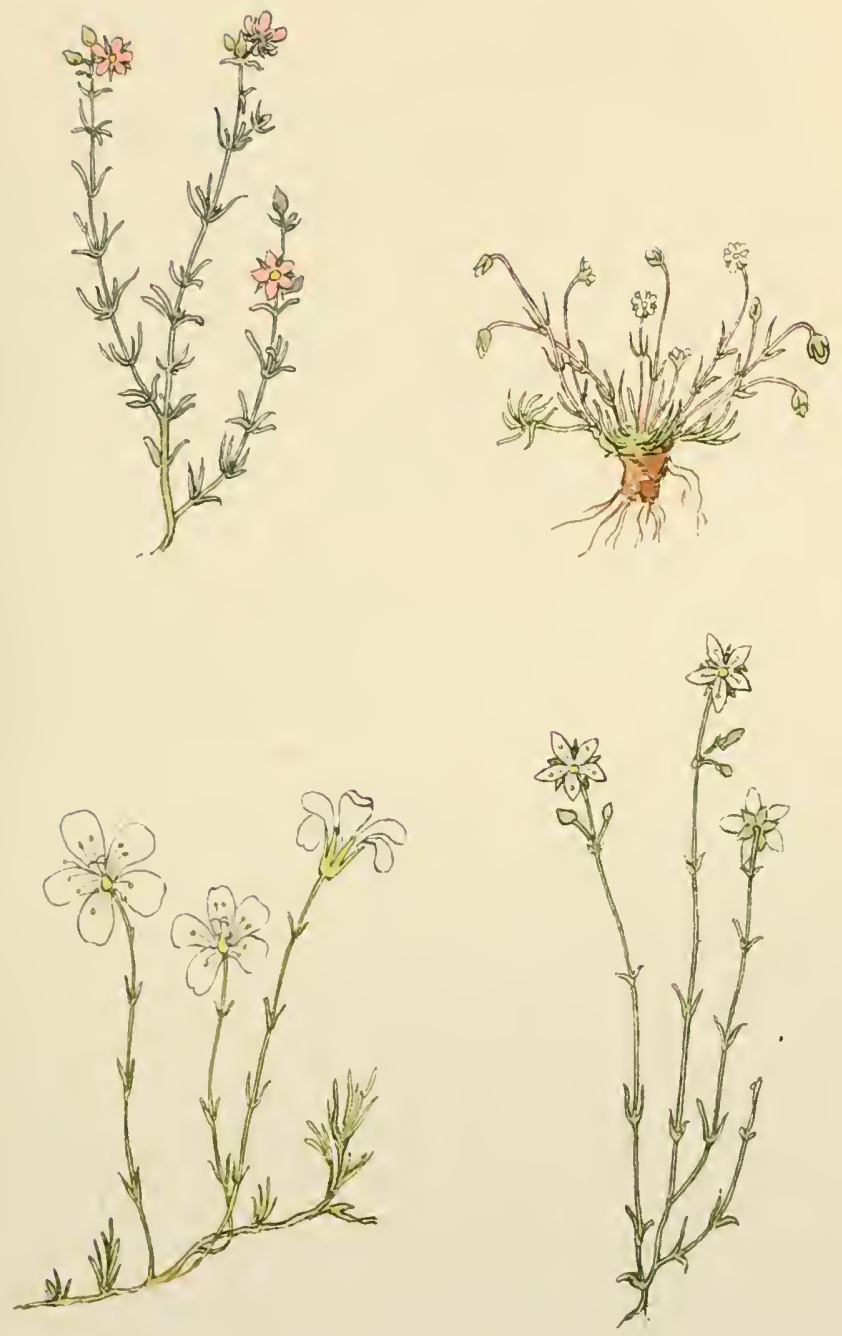

Spergularia rubra.

Sagina Linnai. Alsine Jaricifolis. 


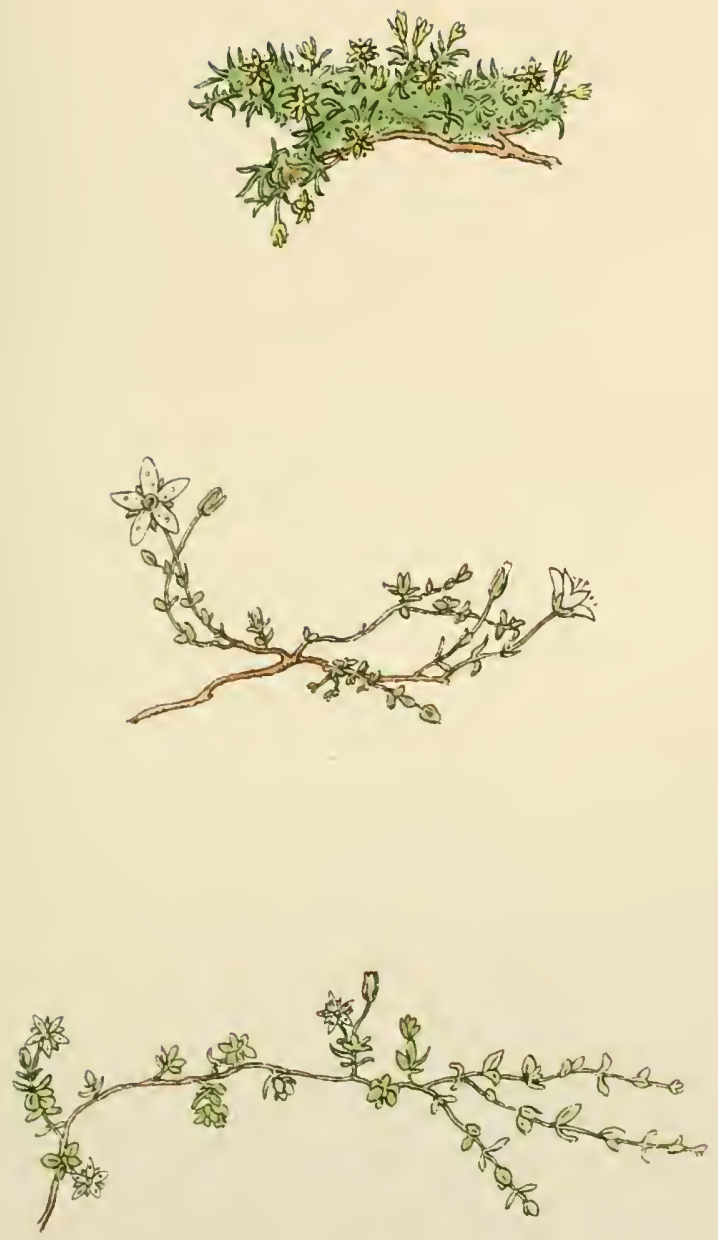

Alsine Cherleri.

Arenaria ciliata.

Arenaria biflora. 



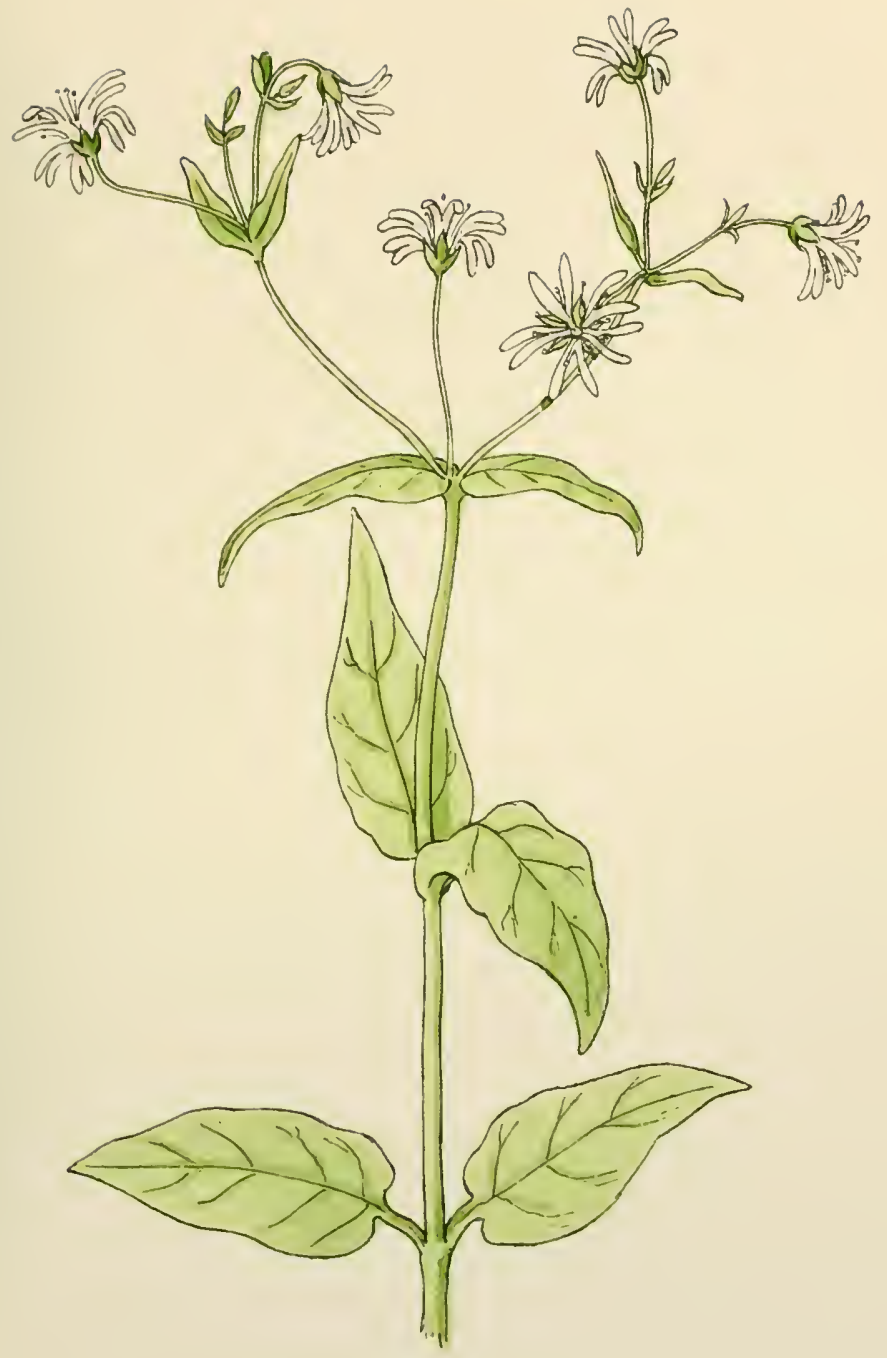

Stellaria nemorum. 


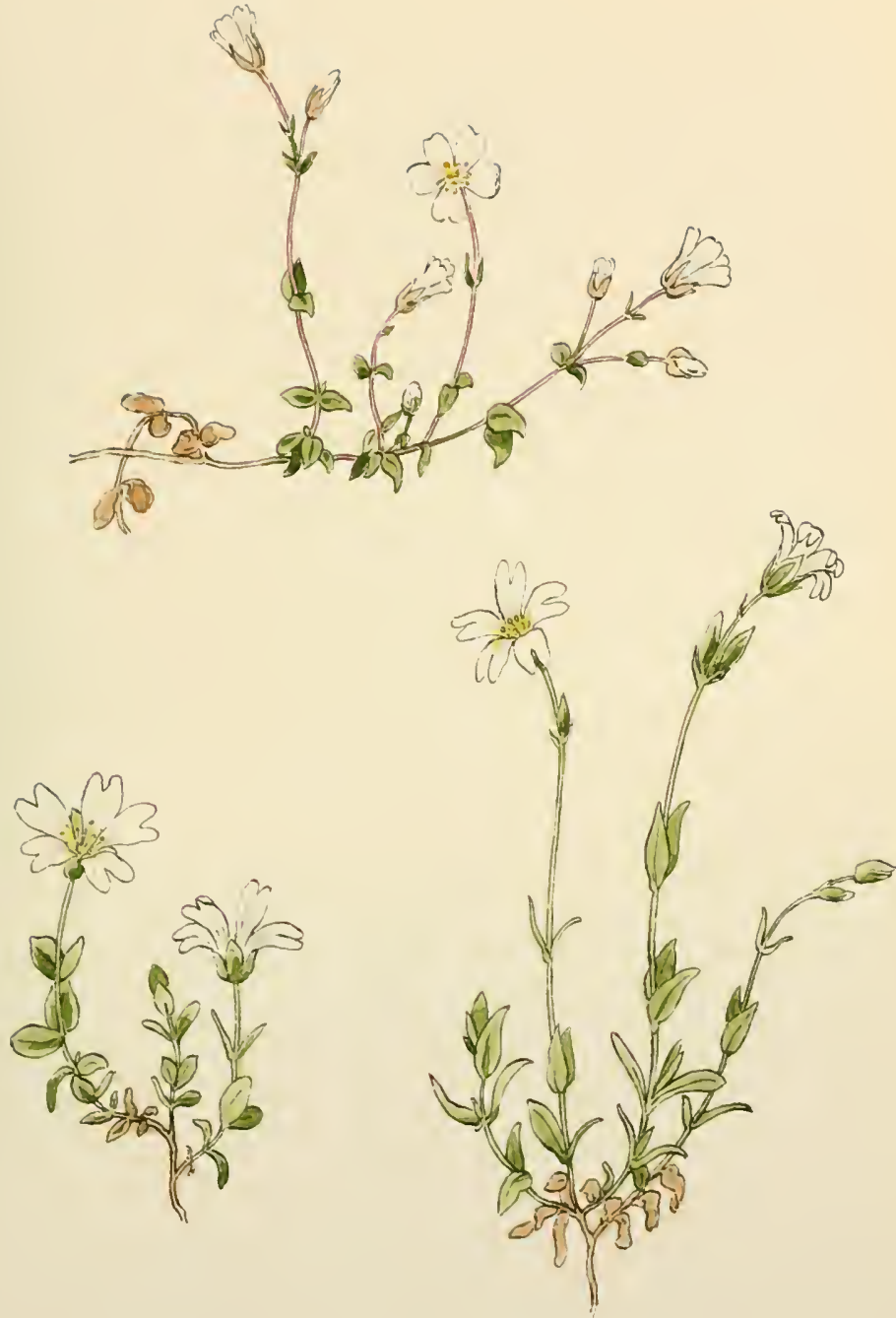

Cerastium trigynum.

Cerastium alpinum.

Cerastium arvense. 

XLVI.

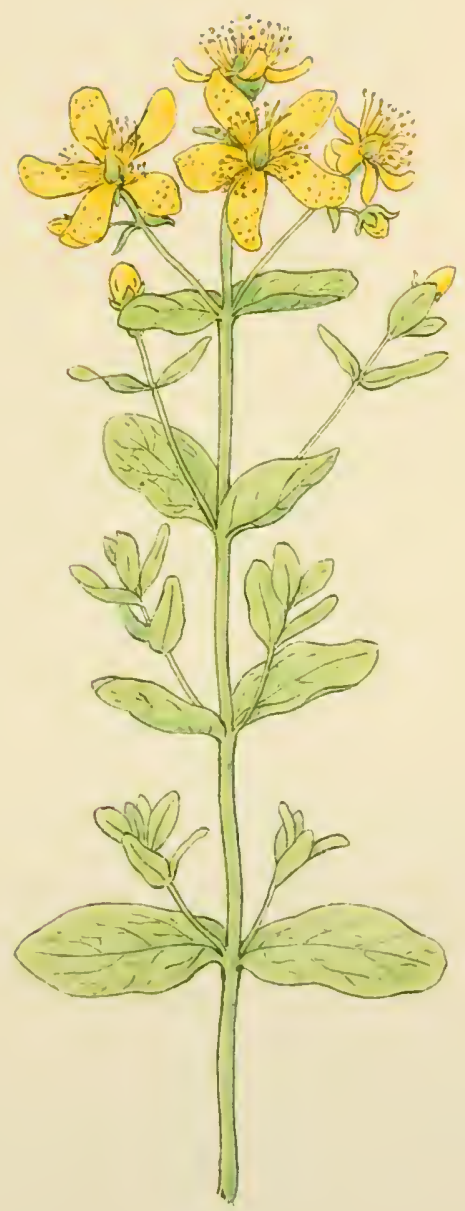

Hypericum quadrangulum. 

XLV]I.

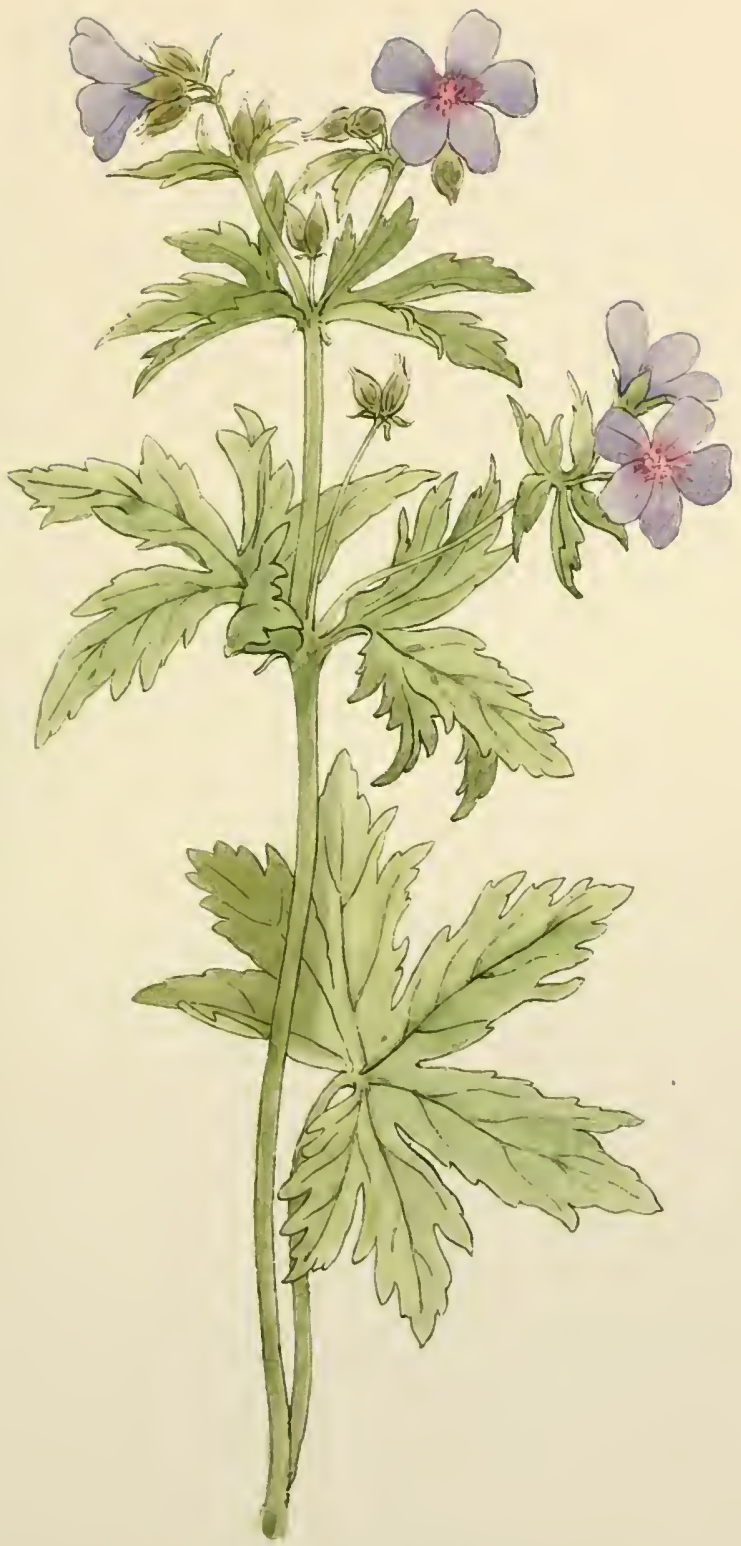

Gexanium sylvaticum. 



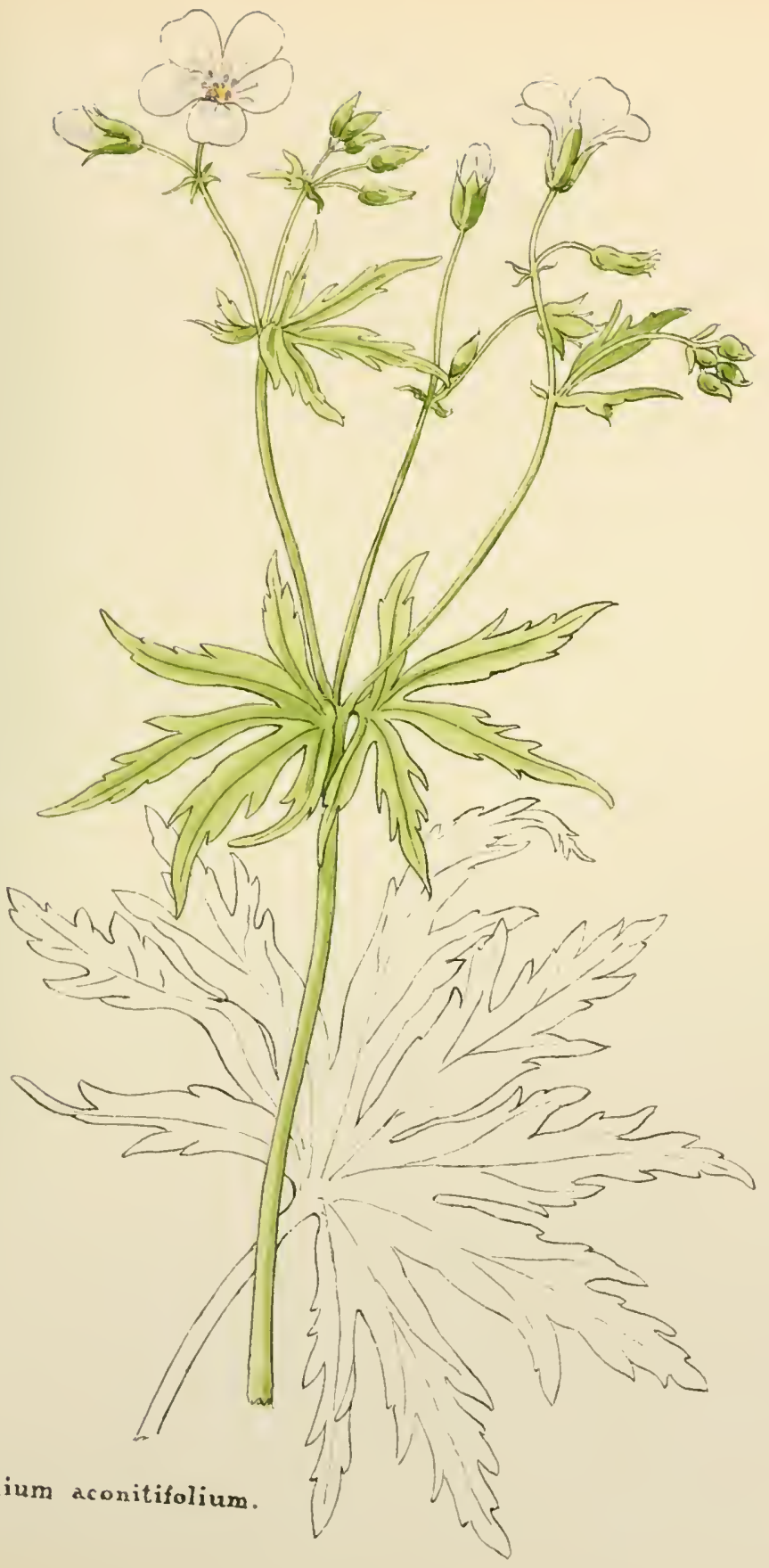

XLVIII. 



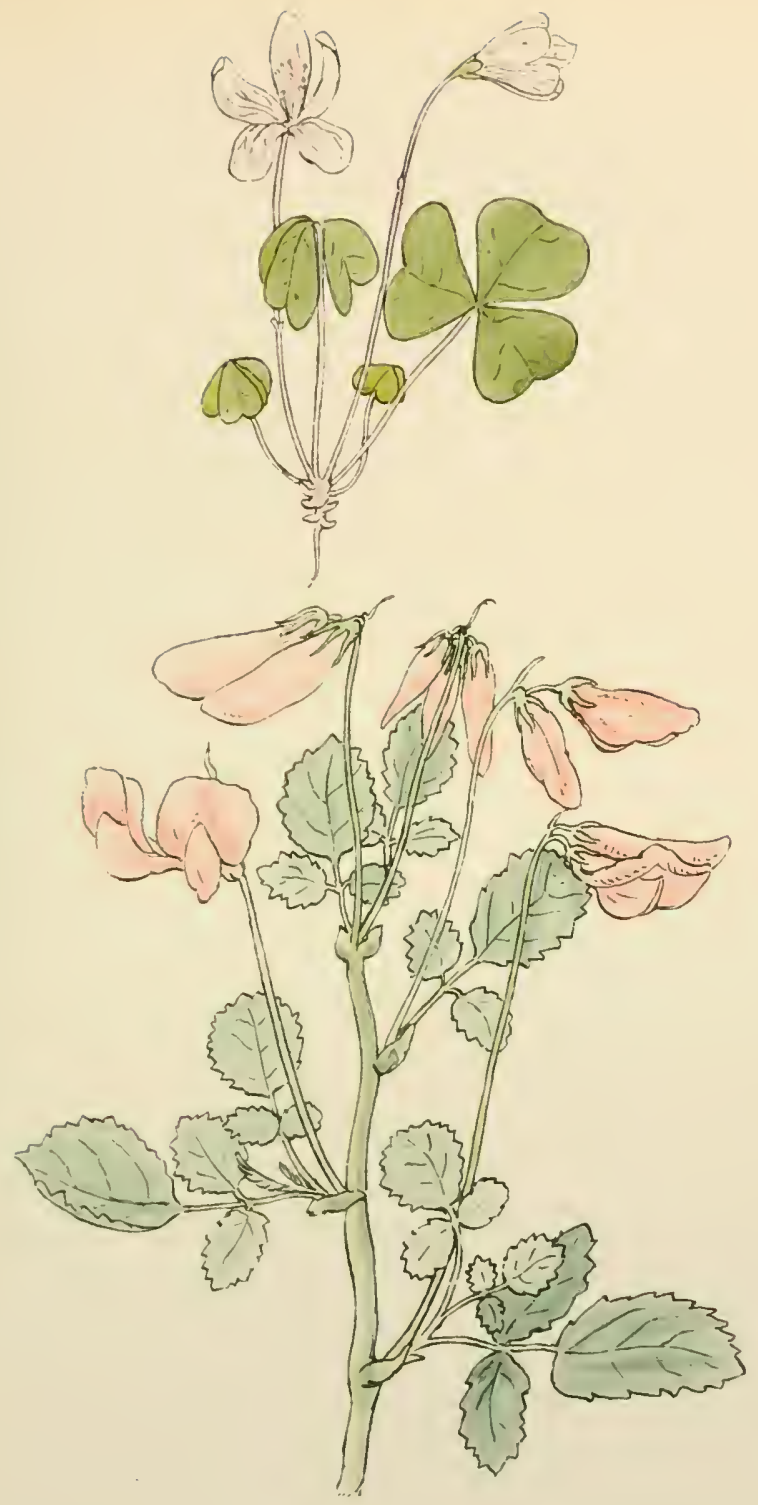

XLIX.

Oxalis acetosella.

Ononis rotundifolia. 


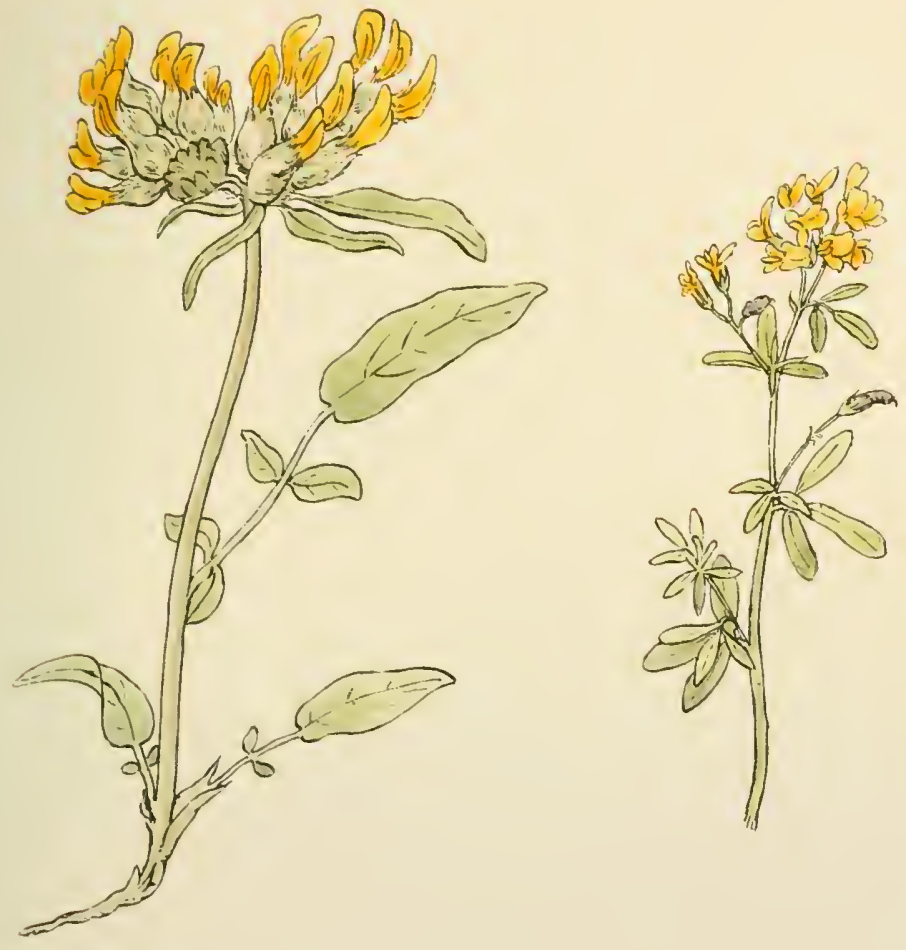

Anthyllis Vulneraria. 

LI.

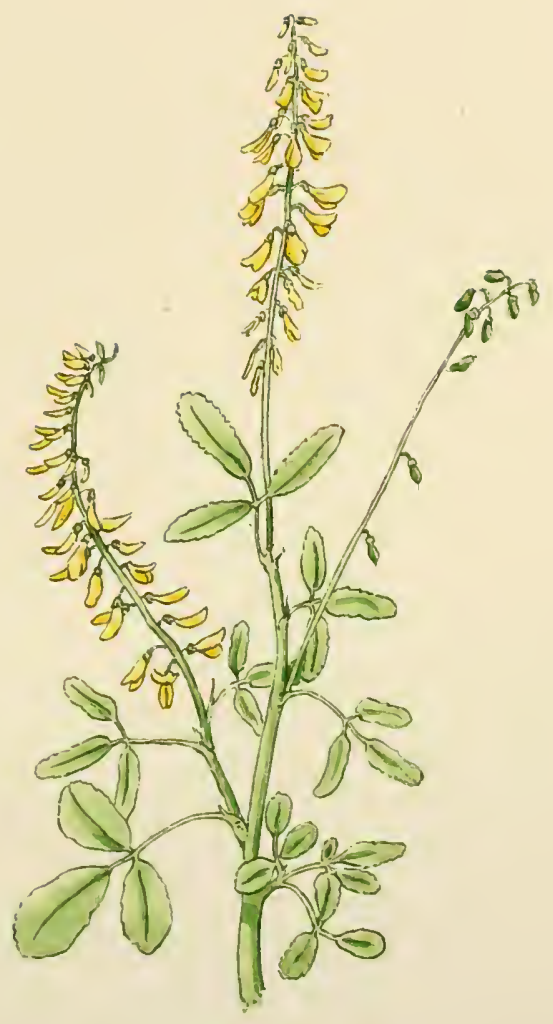

Melilotus arvensis. 



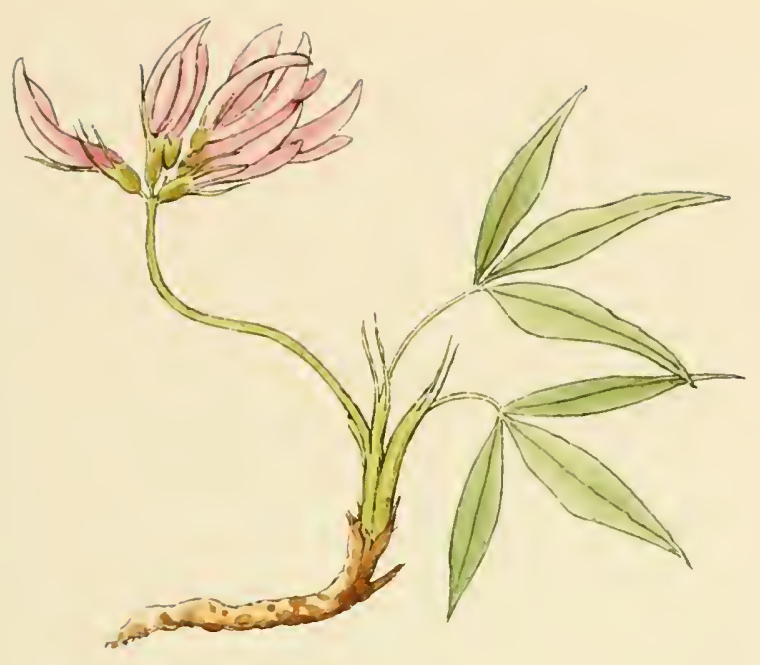

LII.

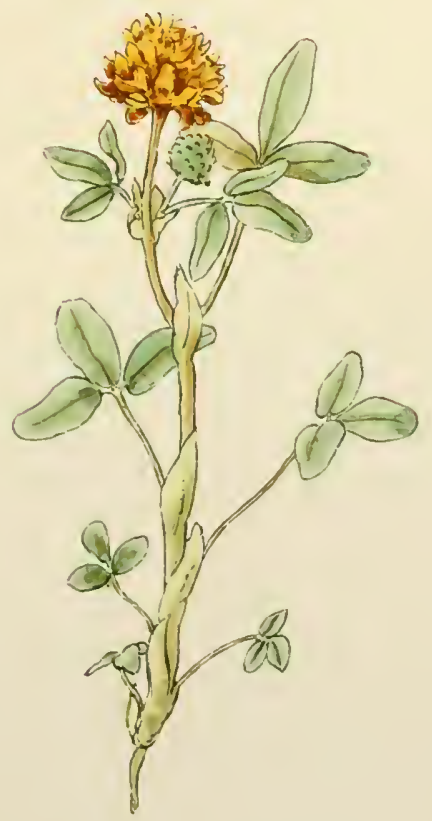

Trifolium alpinura.

Trifolium badium. 

LIII.

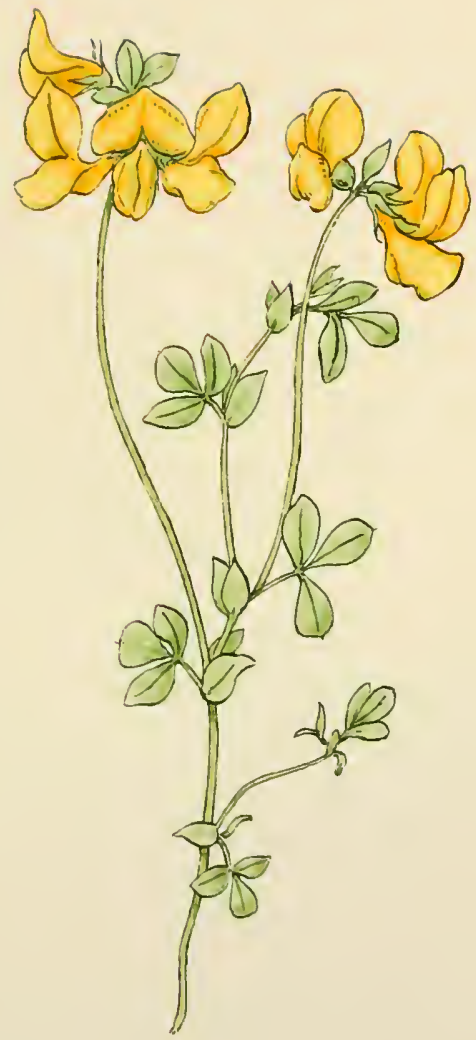

Lotus corniculatus. 

LIV.

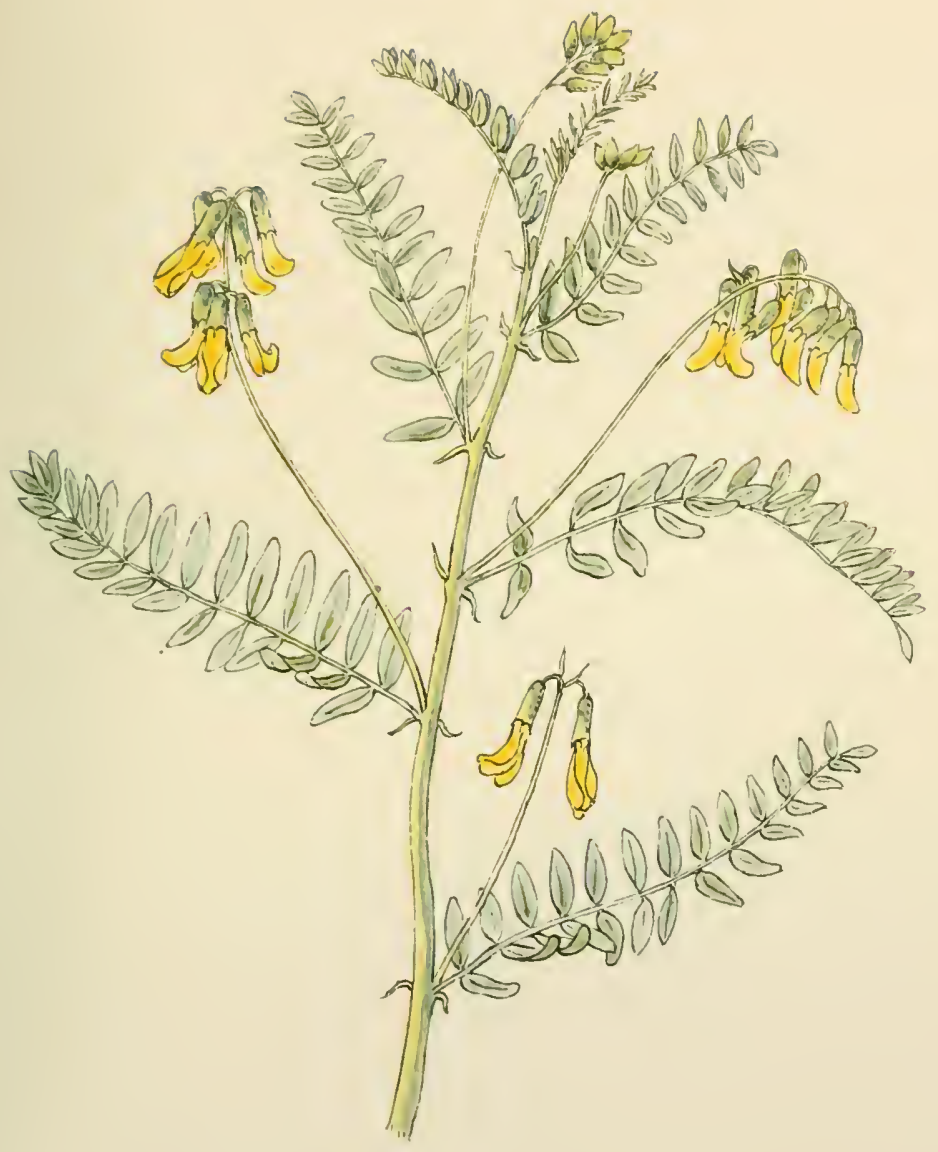

Phaca alpina. 

LV.

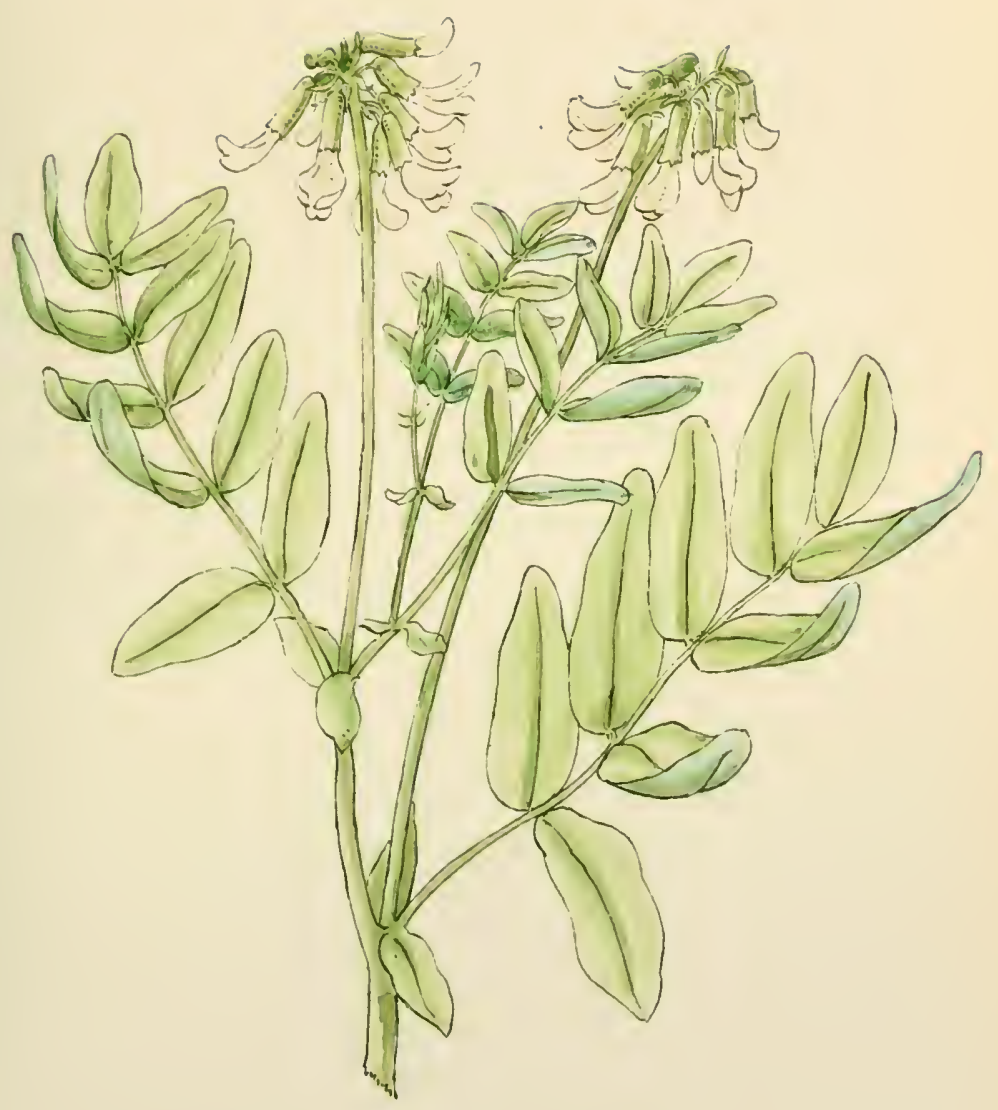

Phaca frigida. 

LVI

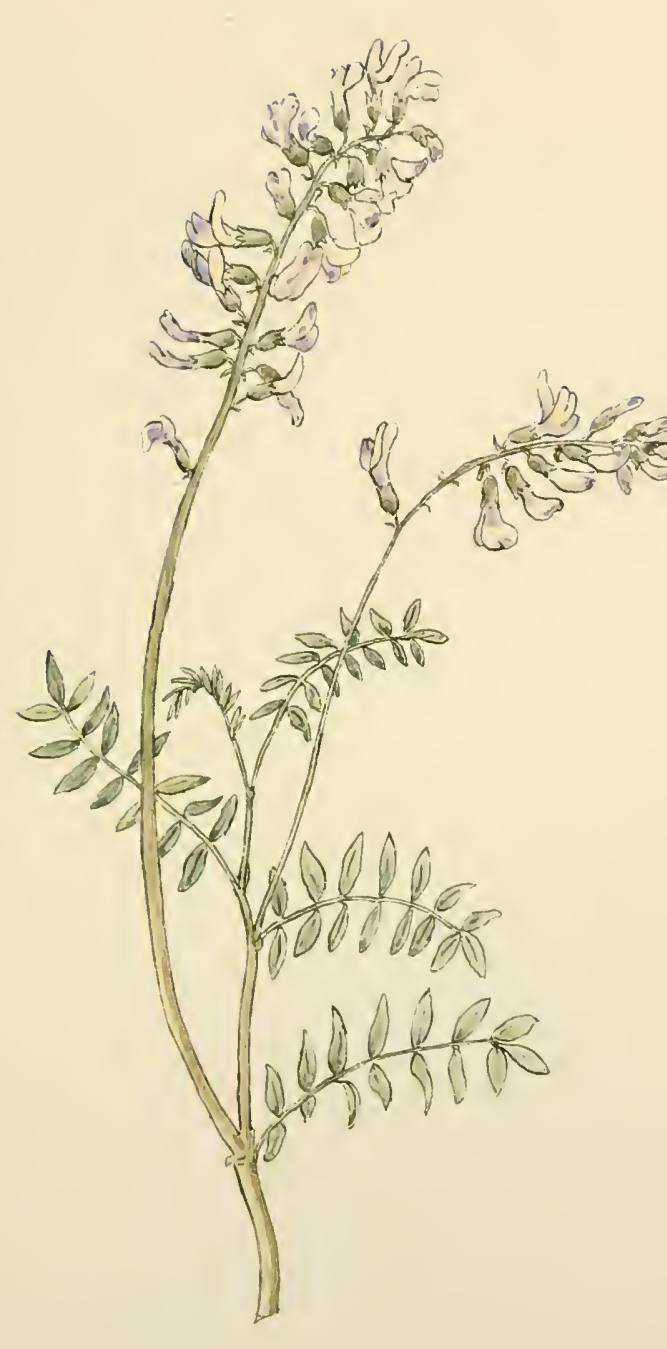




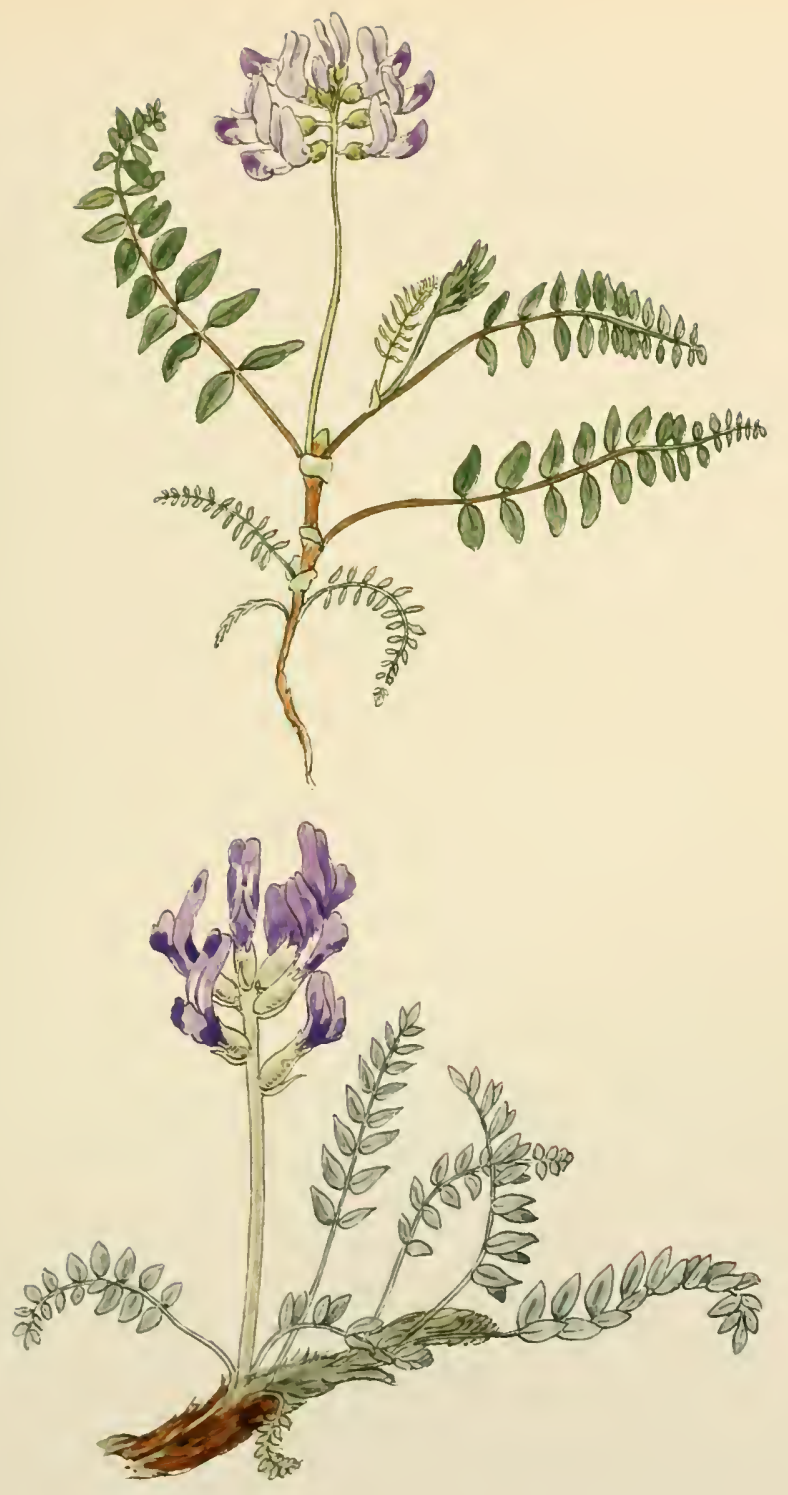

L.VII. 



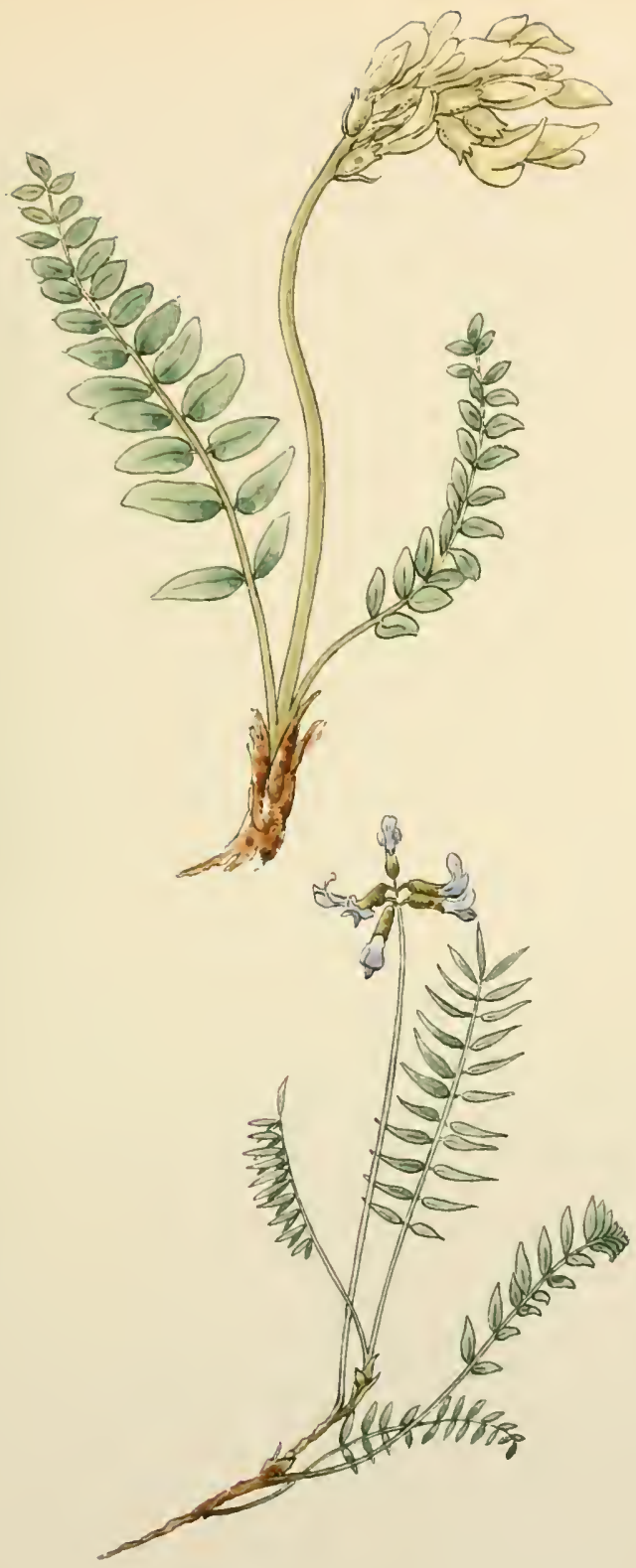

LVIII .

Oxytropis campestris.

Oxytropis montana. 

LIX.

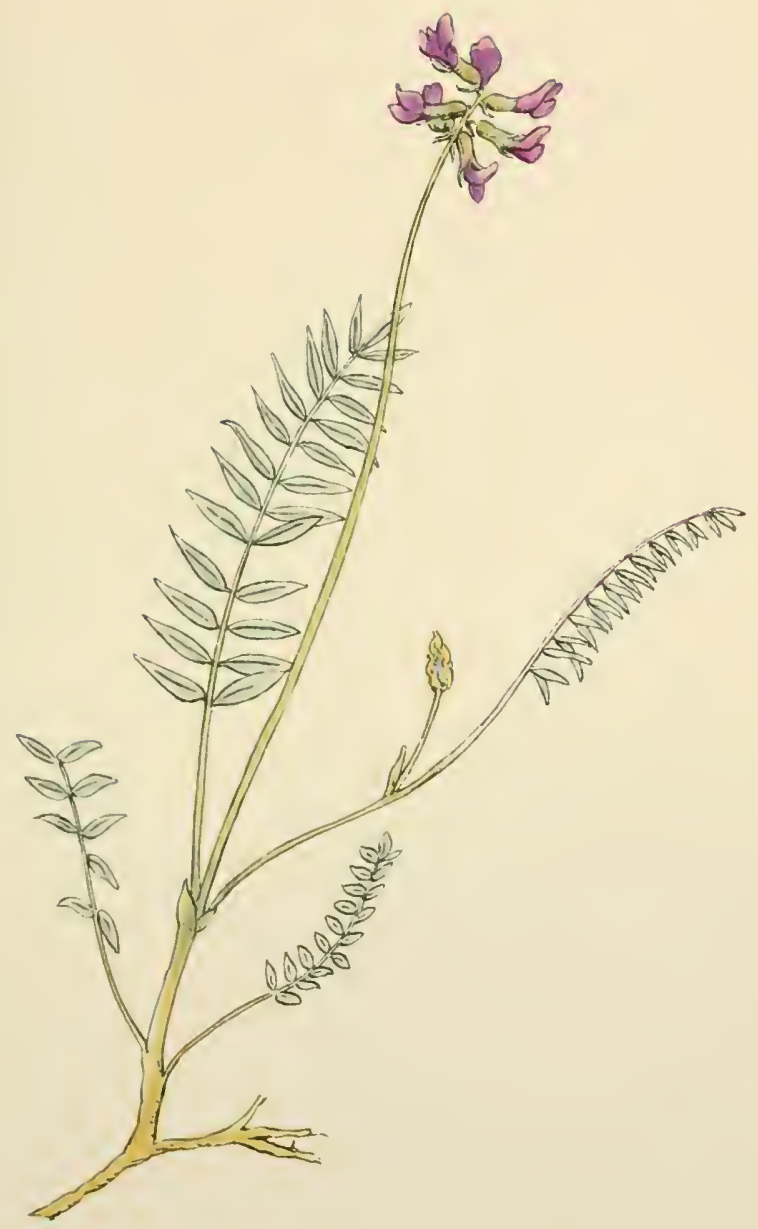

Oxytropis lapponica. 

LX.

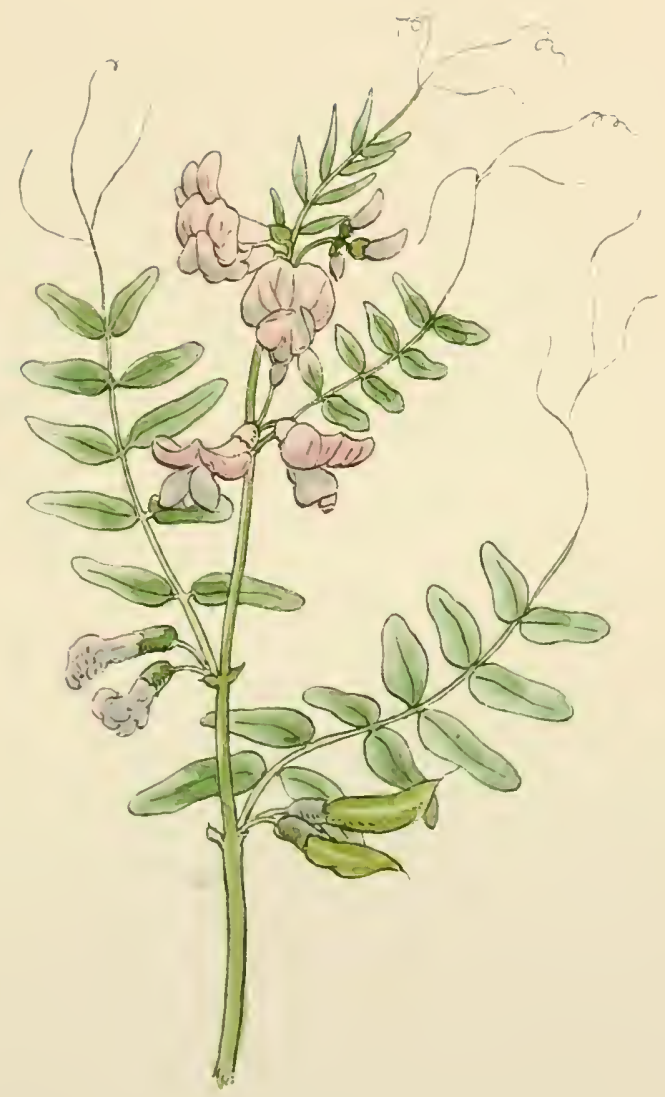

Vicia sepium. 

LXI.

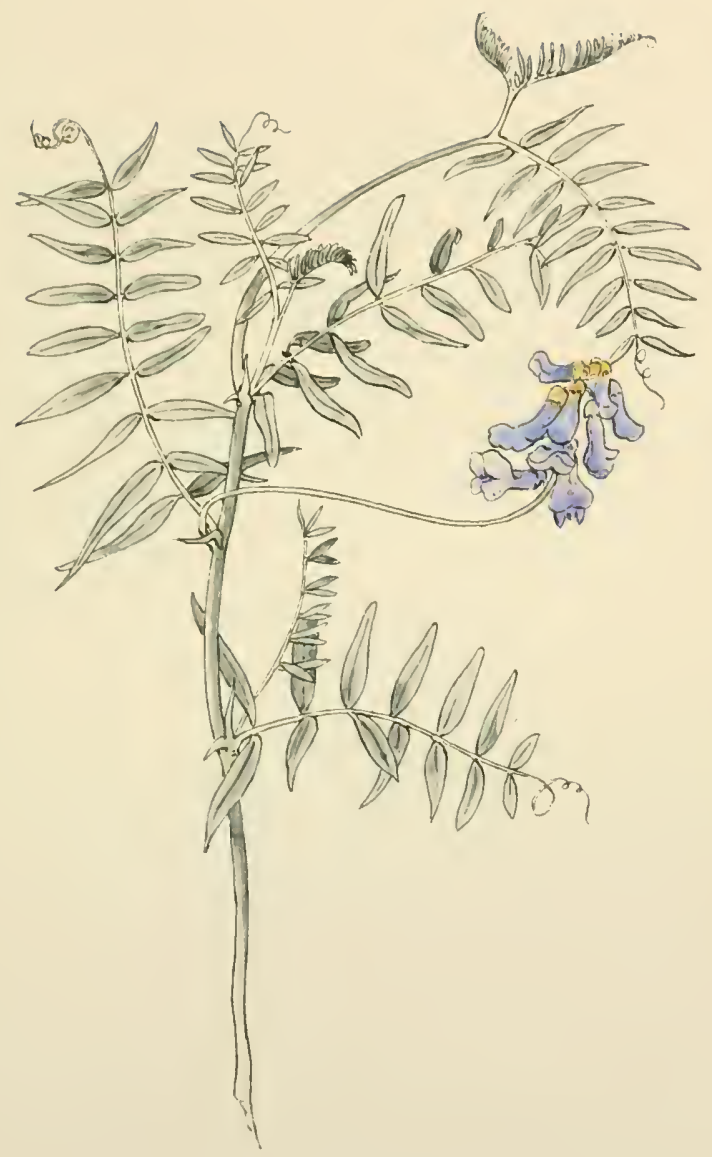

Vicia Cracca. 

LXXII.

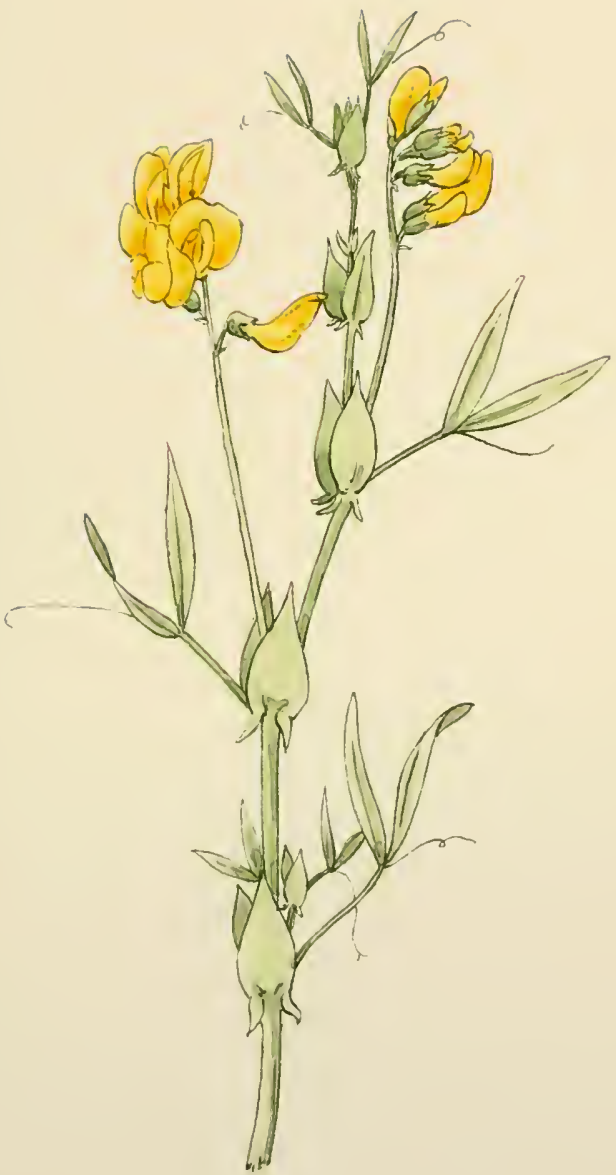

Lathyrus pratensis. 

LXIII.

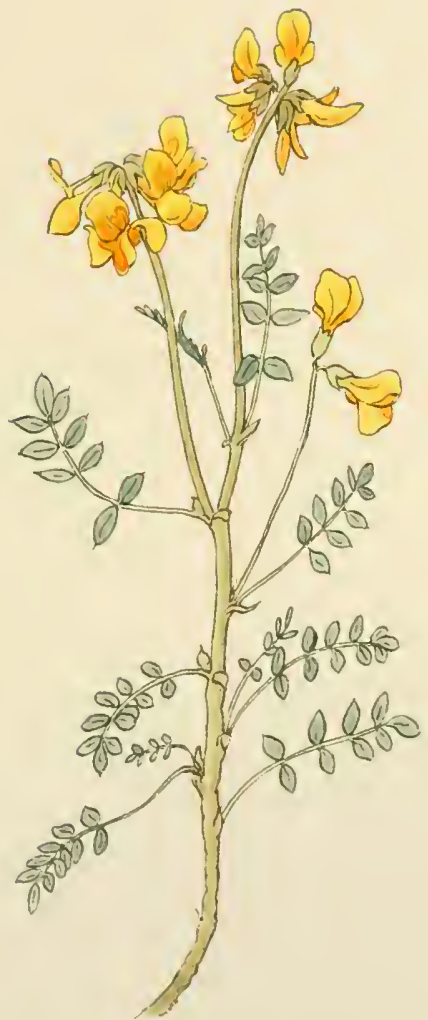

Hippocrepis comosa. 

LXIV.

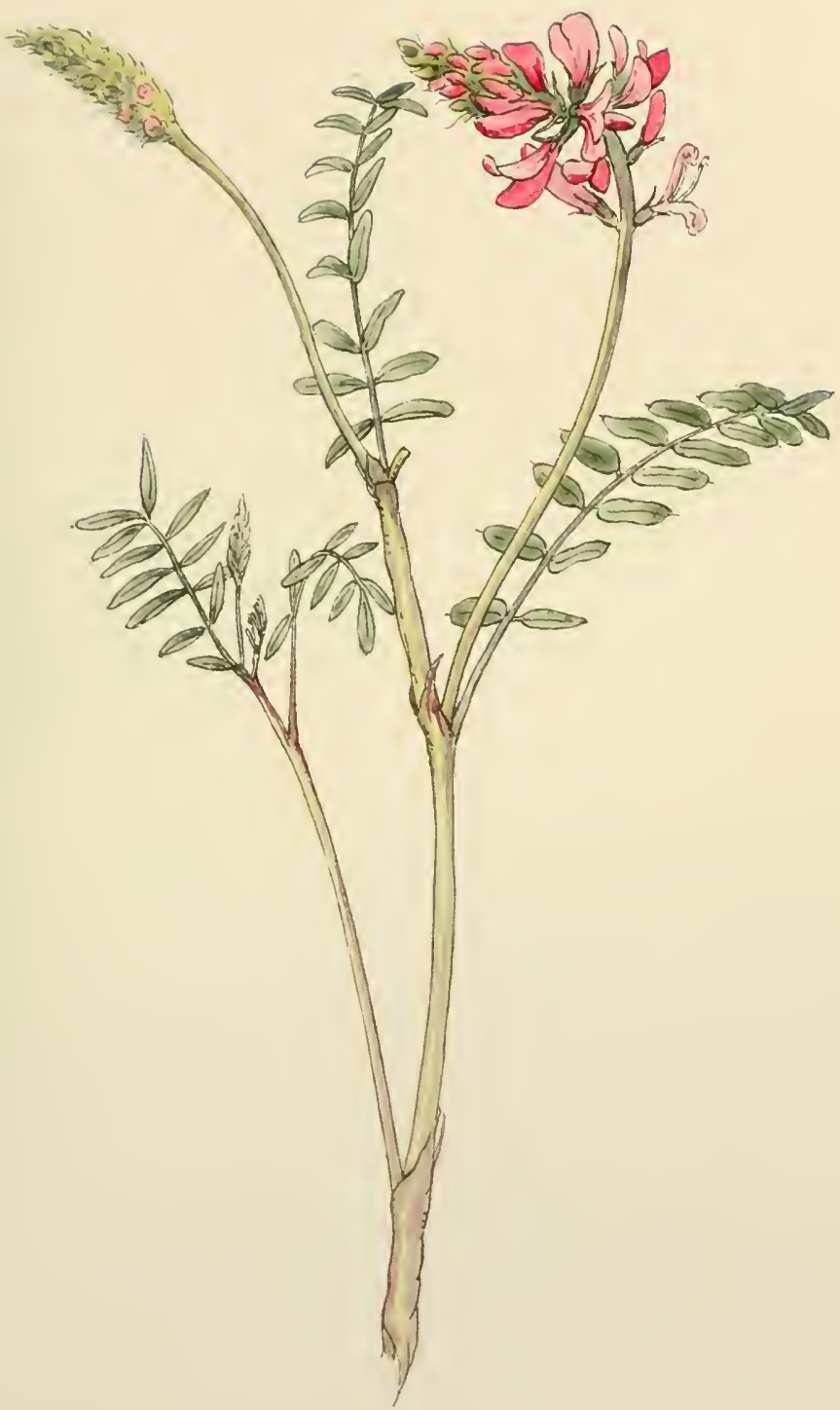

Onobrychis montana. 

LXV.

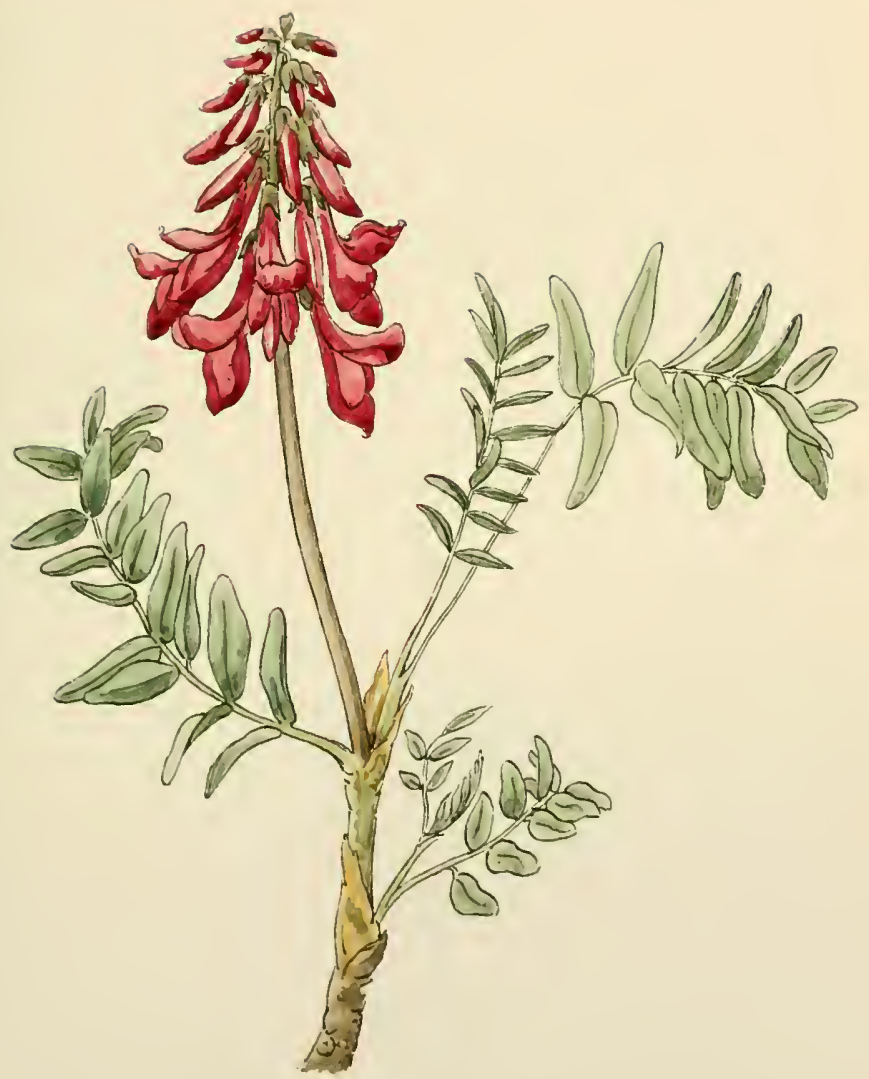

Hedysarum obscurum. 

LXVI.

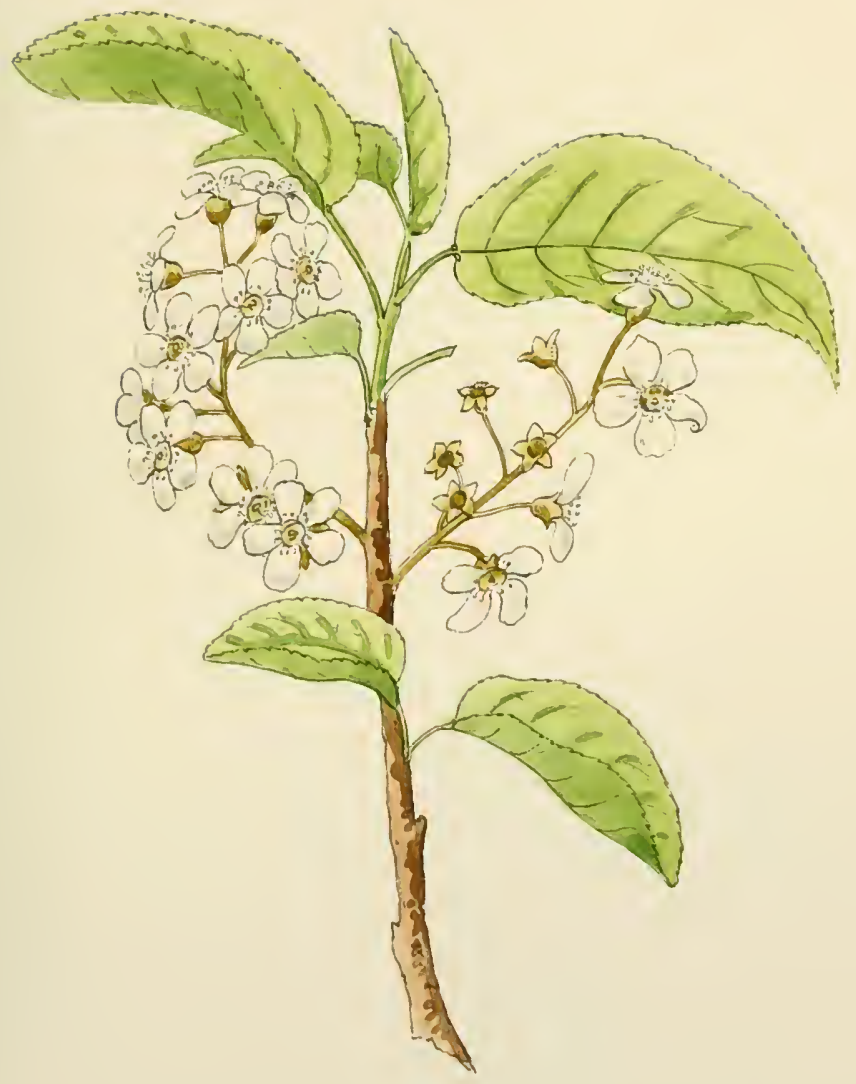

Cerasus Padus. 



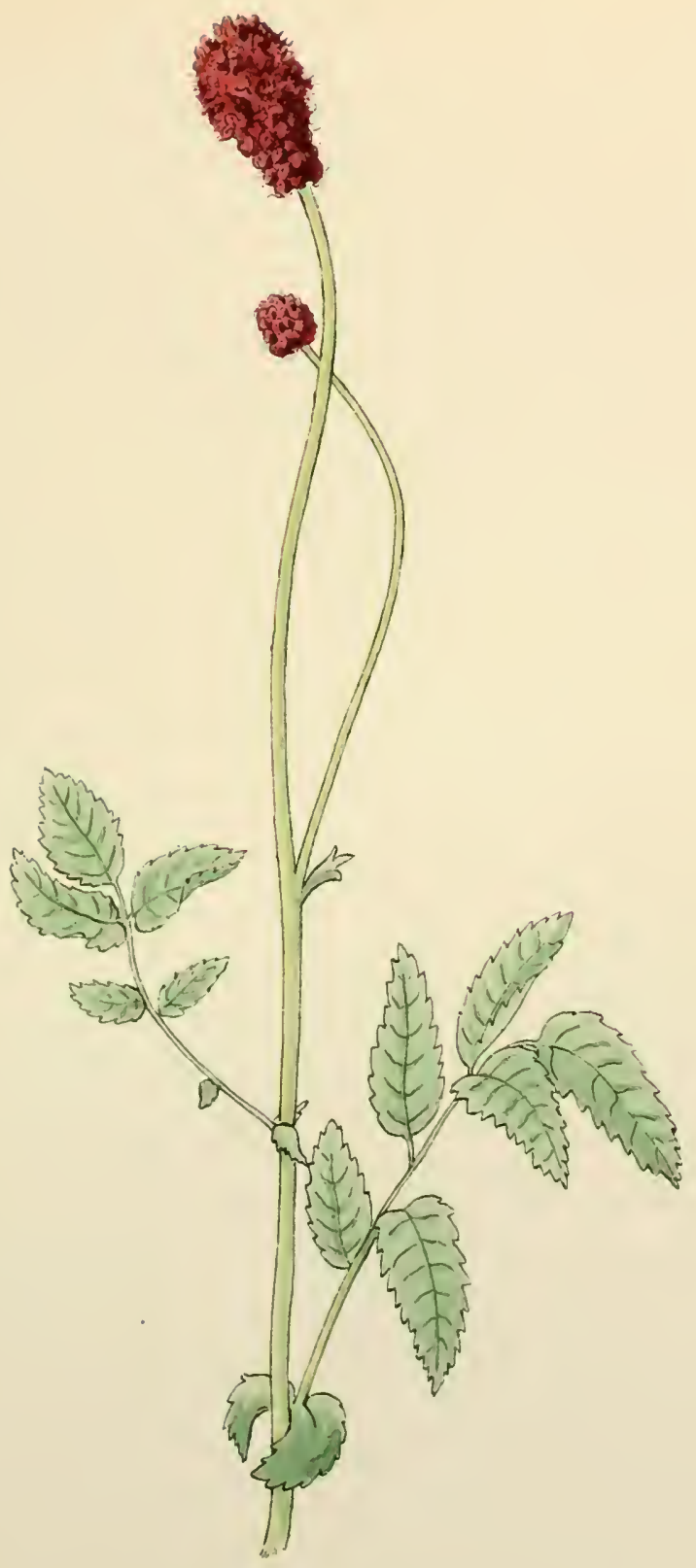

IXVIII

Sanguisorba officinalis. 
LXVIII.
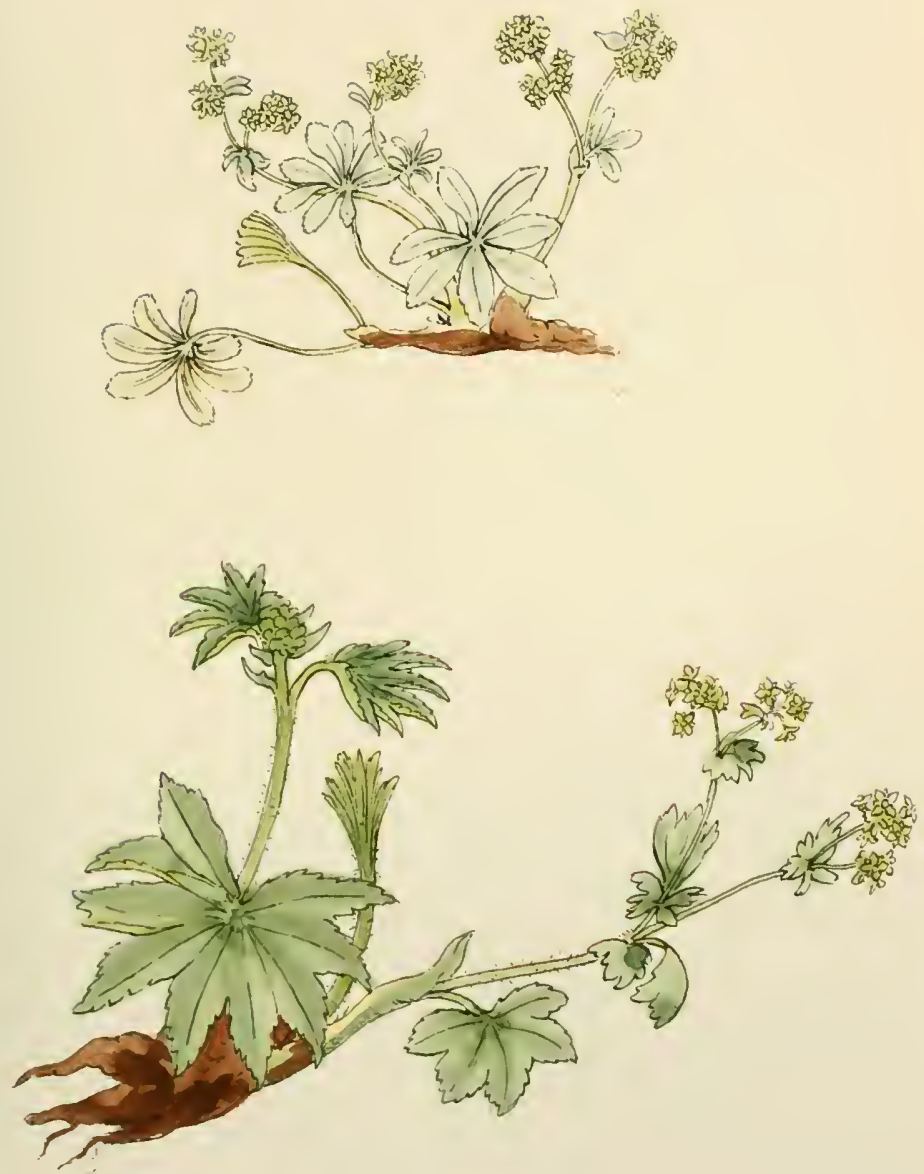

Alchemilla alpina.

Alchemilla vulgaris. 

LXIX.
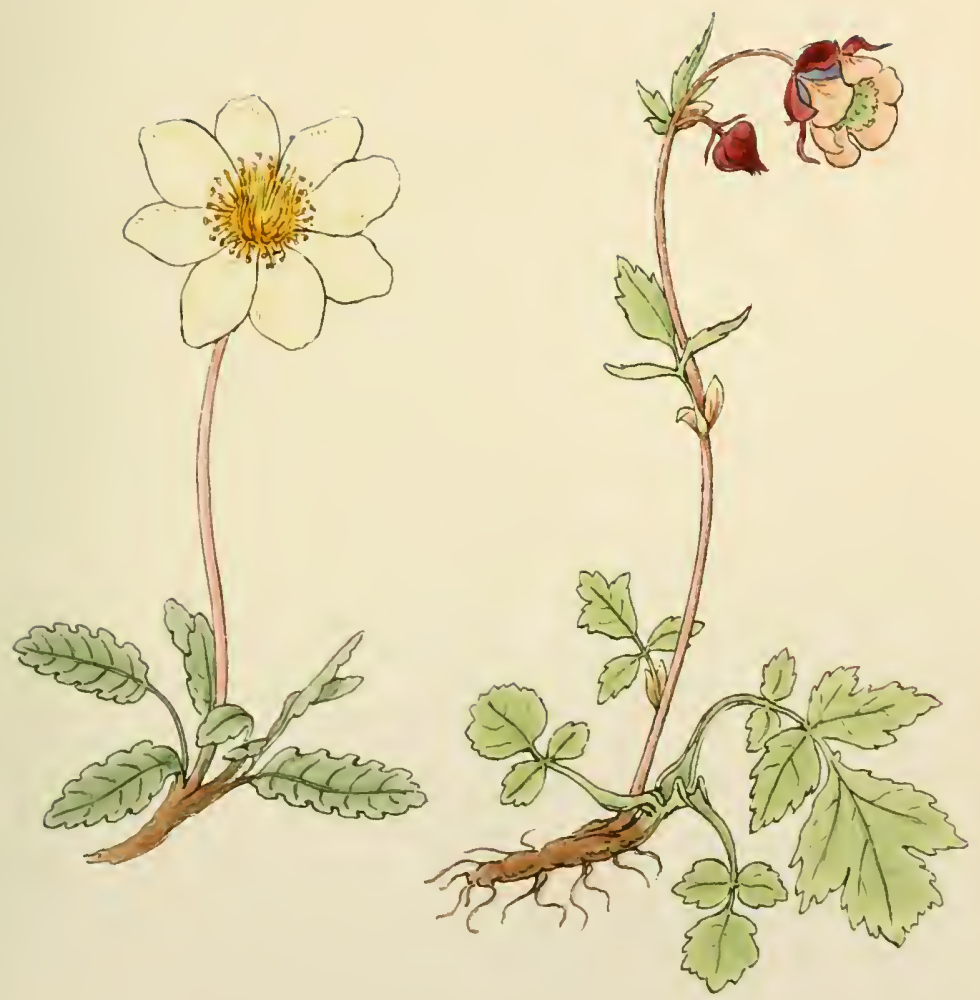

Dryas octopetala.

Geum rivale. 

LXX.

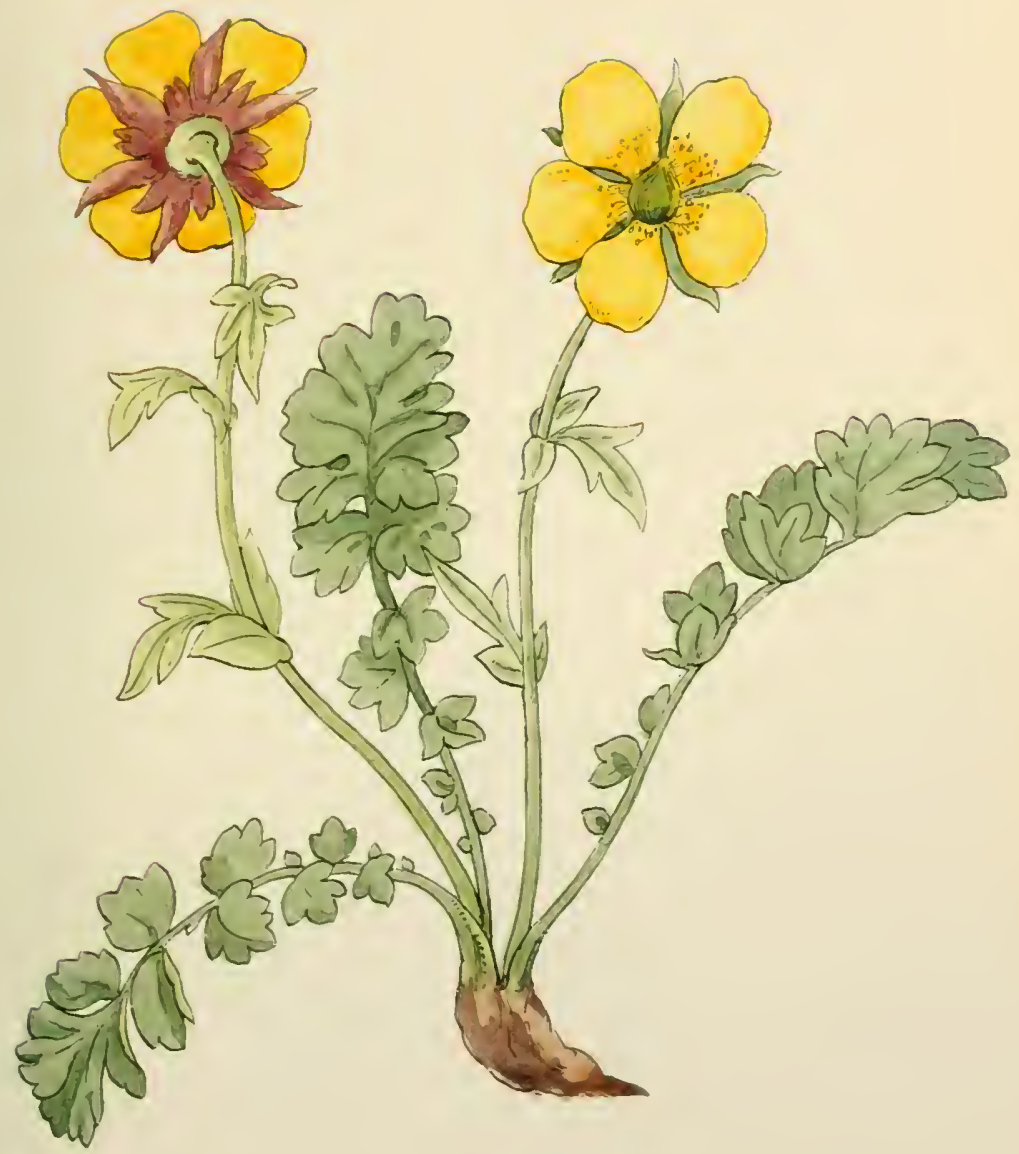

Geum reptans. 

LXXI.

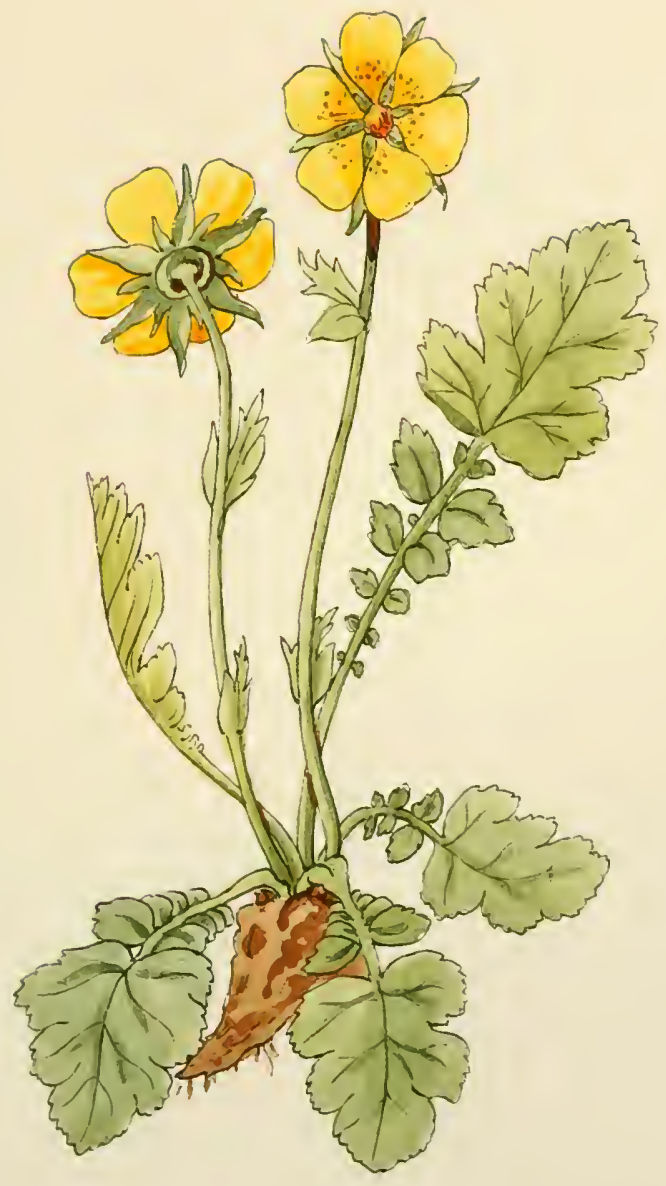

Geum montanum. 

LXXII.
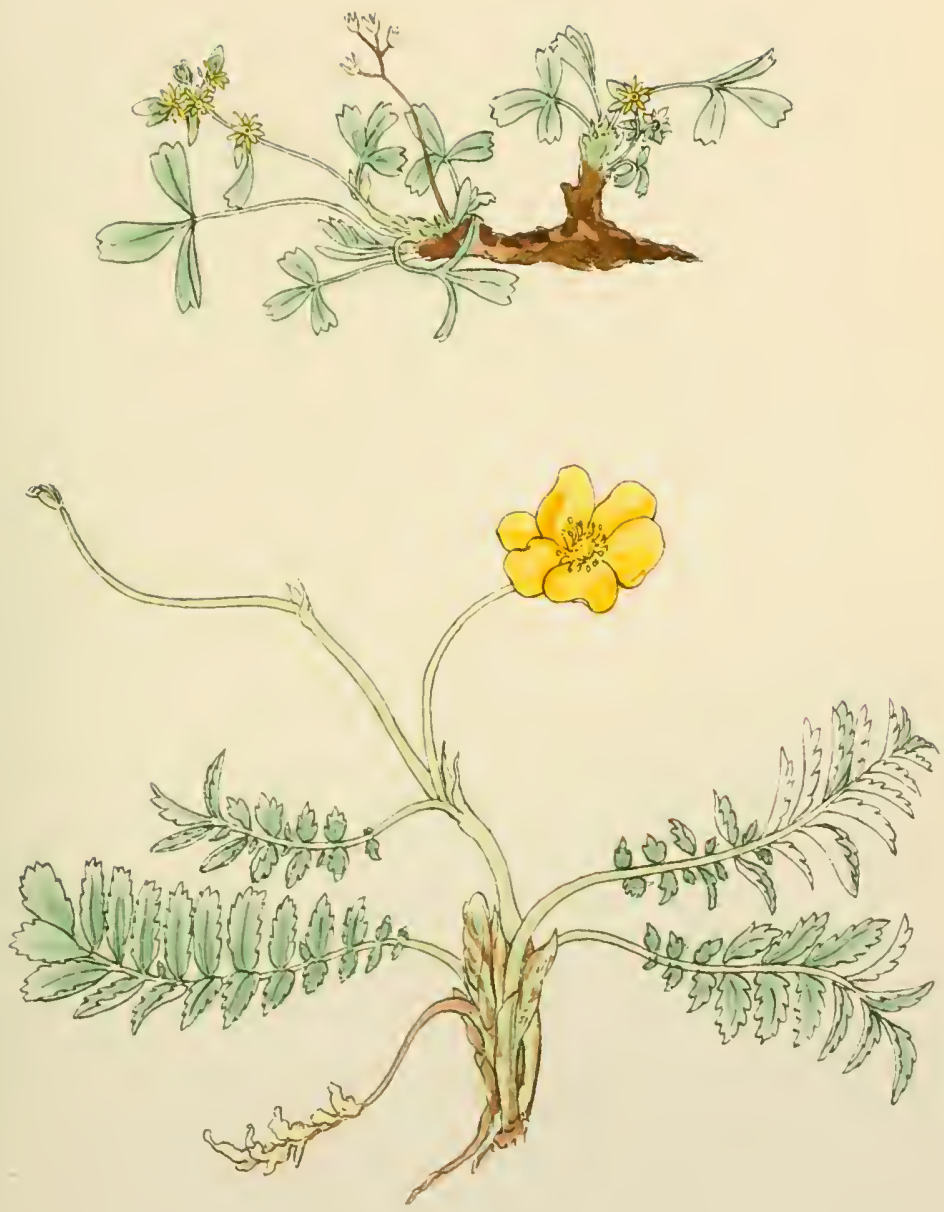

Sibbaldia procumbens.

Potentilla anserina. 

LXXIII.
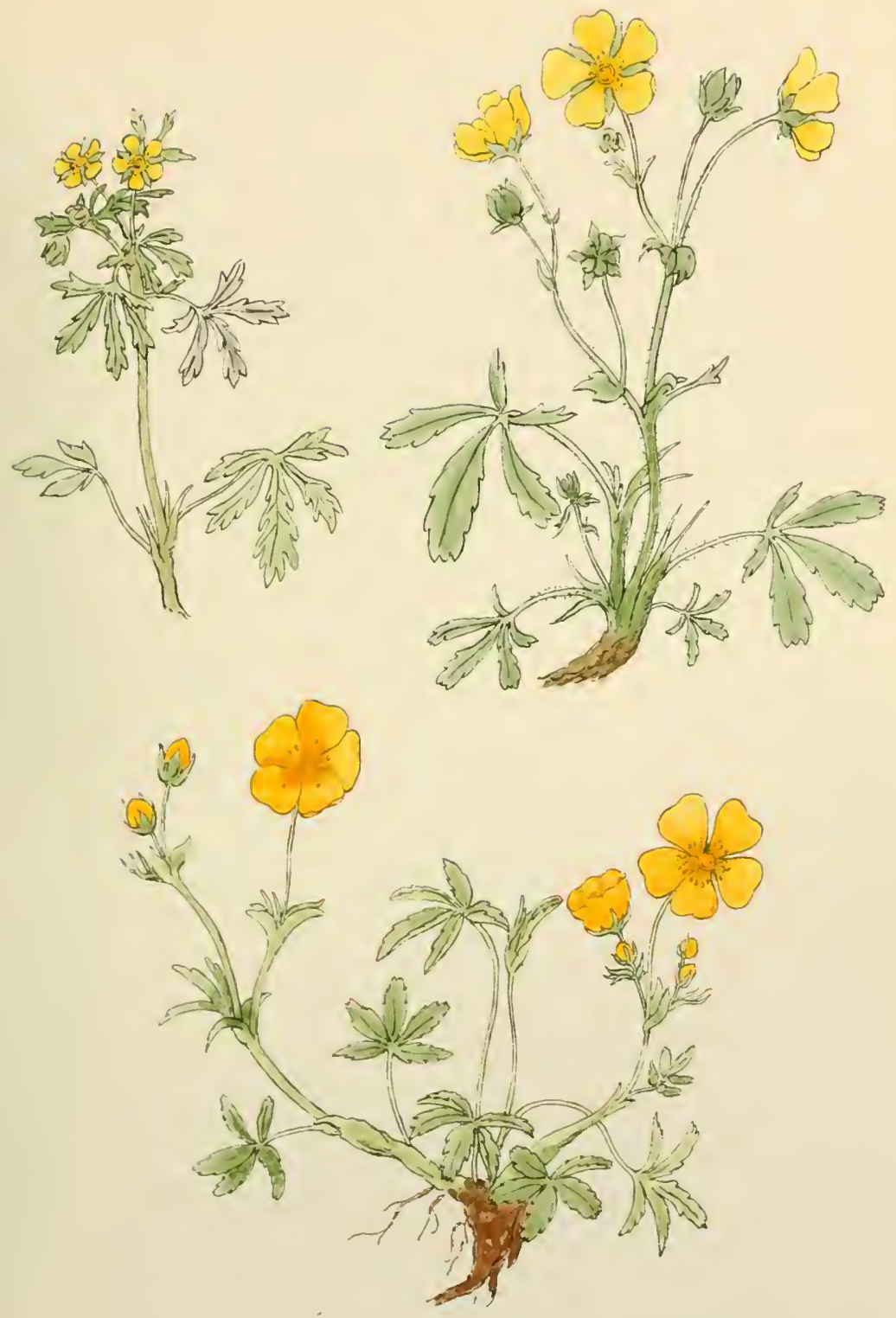

Potentilla verna.

Potentilla argentez.

Potentilla aurea. 



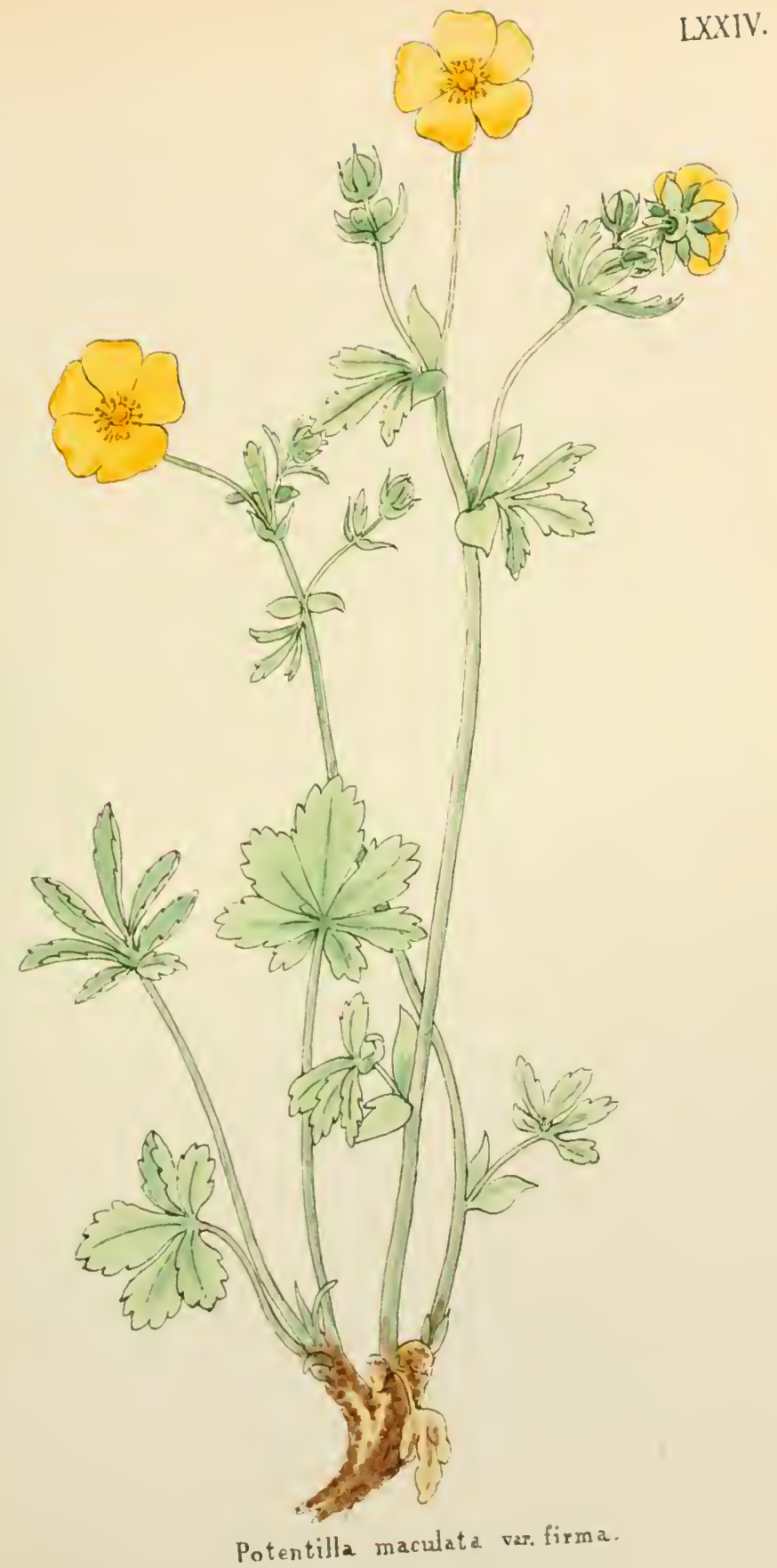



LXXV.

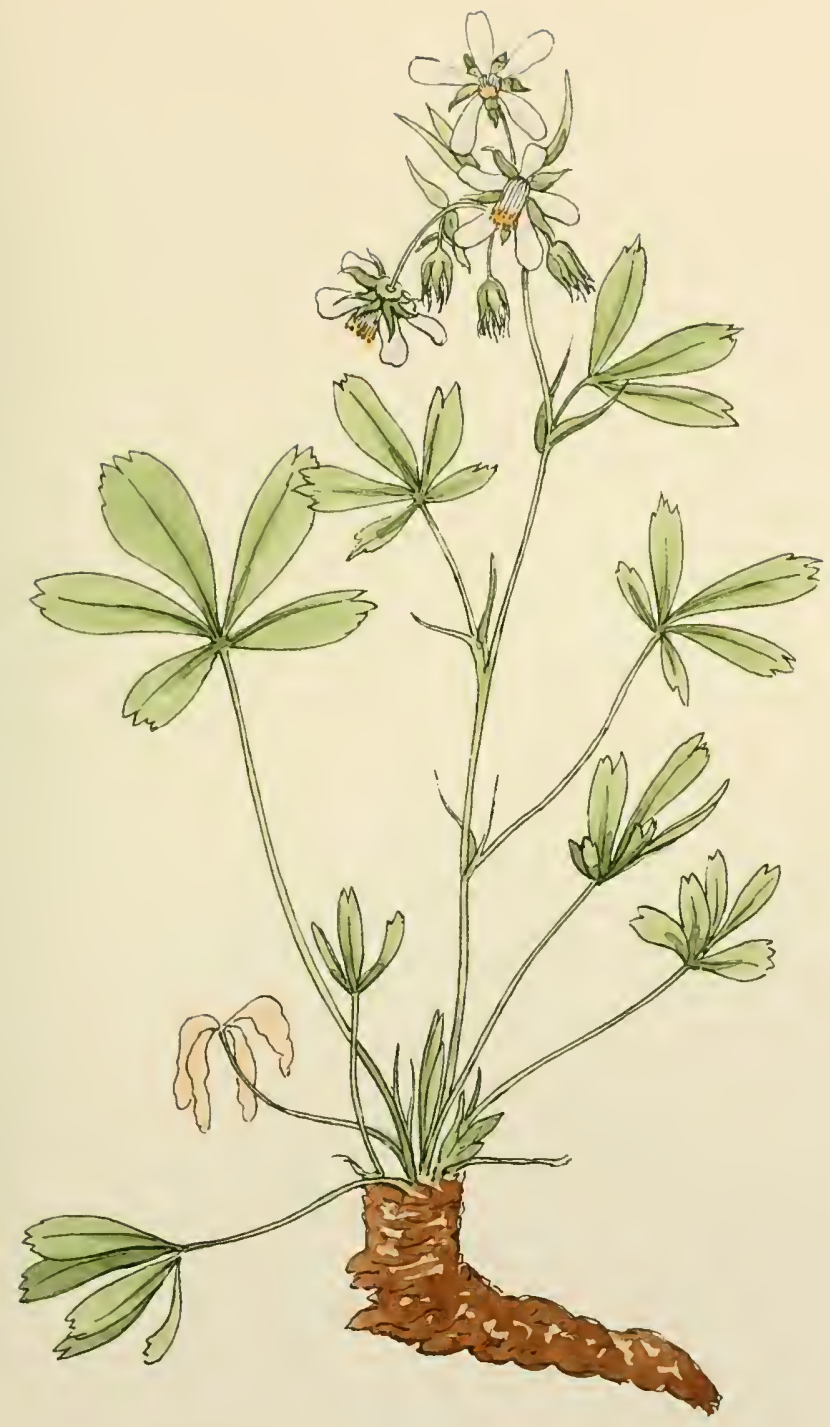

Potentilla caulescens. 

LXXVI.

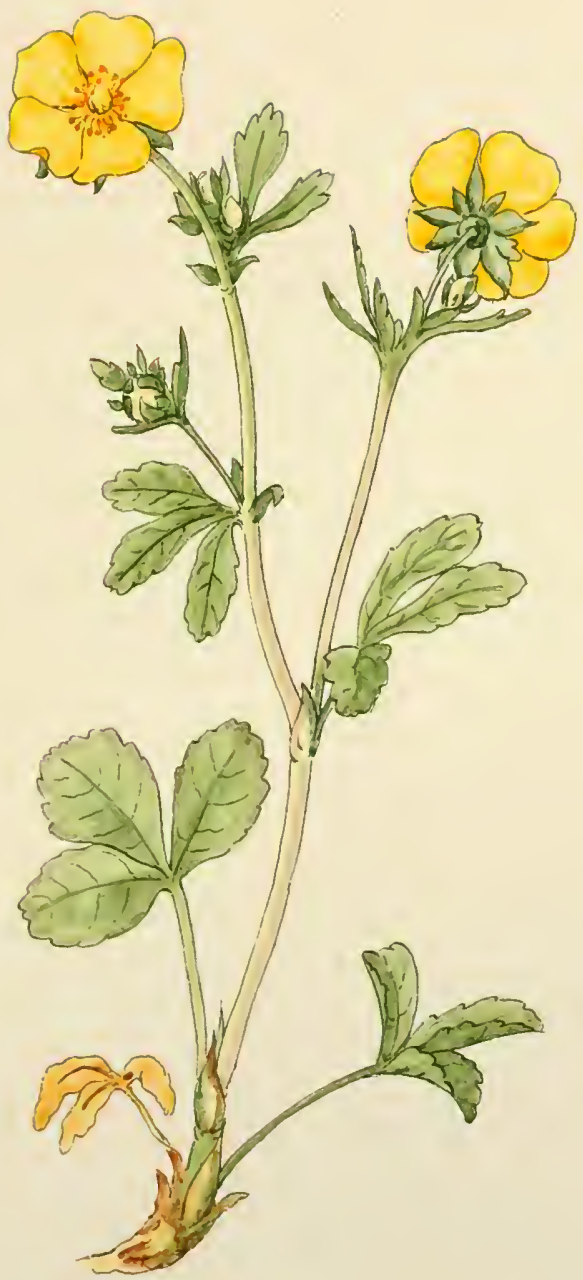

Potentilla grandiflora. 

LXXVII .

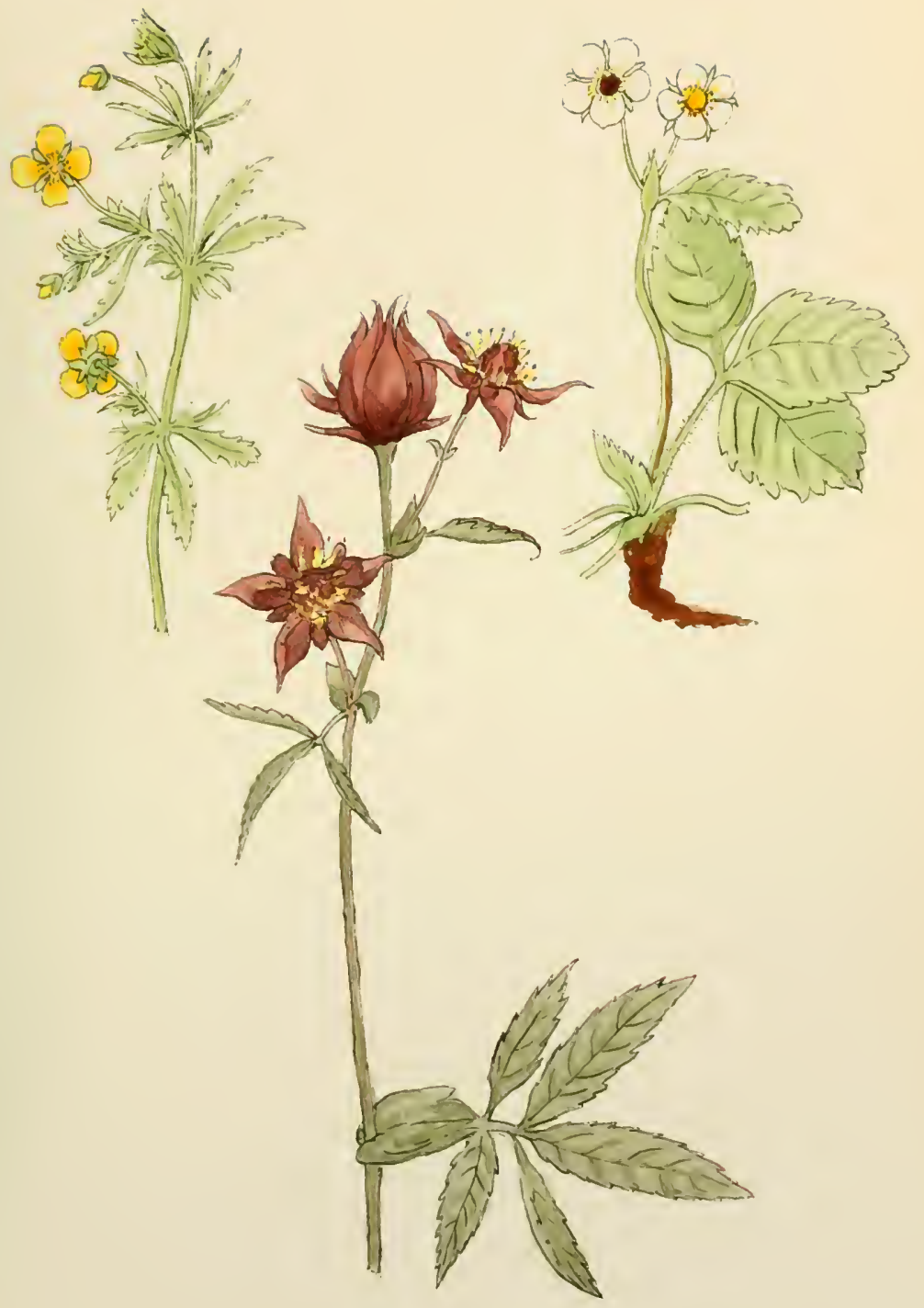

Potentilla Tormentilla. Fragaria vesca.

Comarum palustre. 



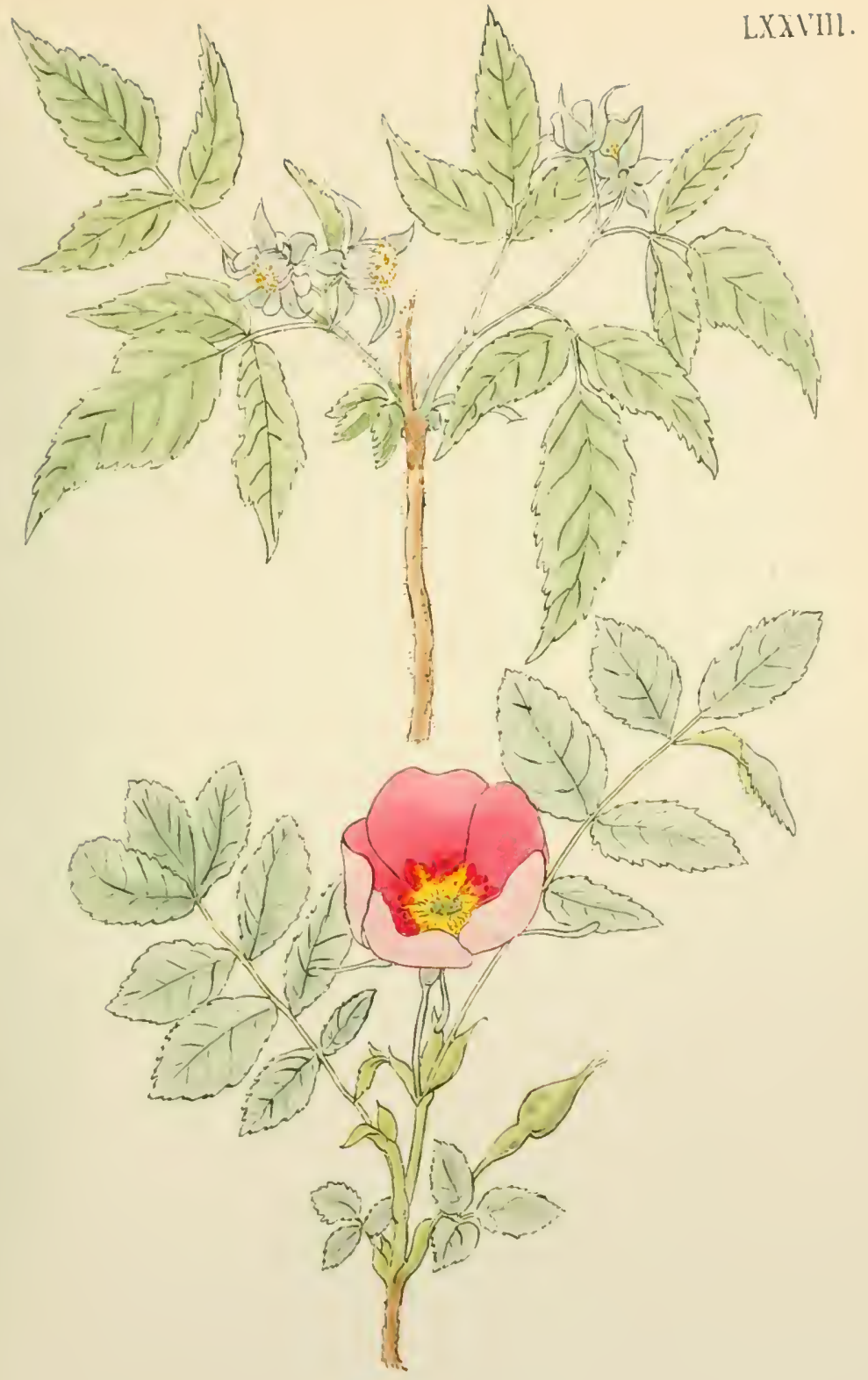

Rubus Idxus.

Rosa alpina. 


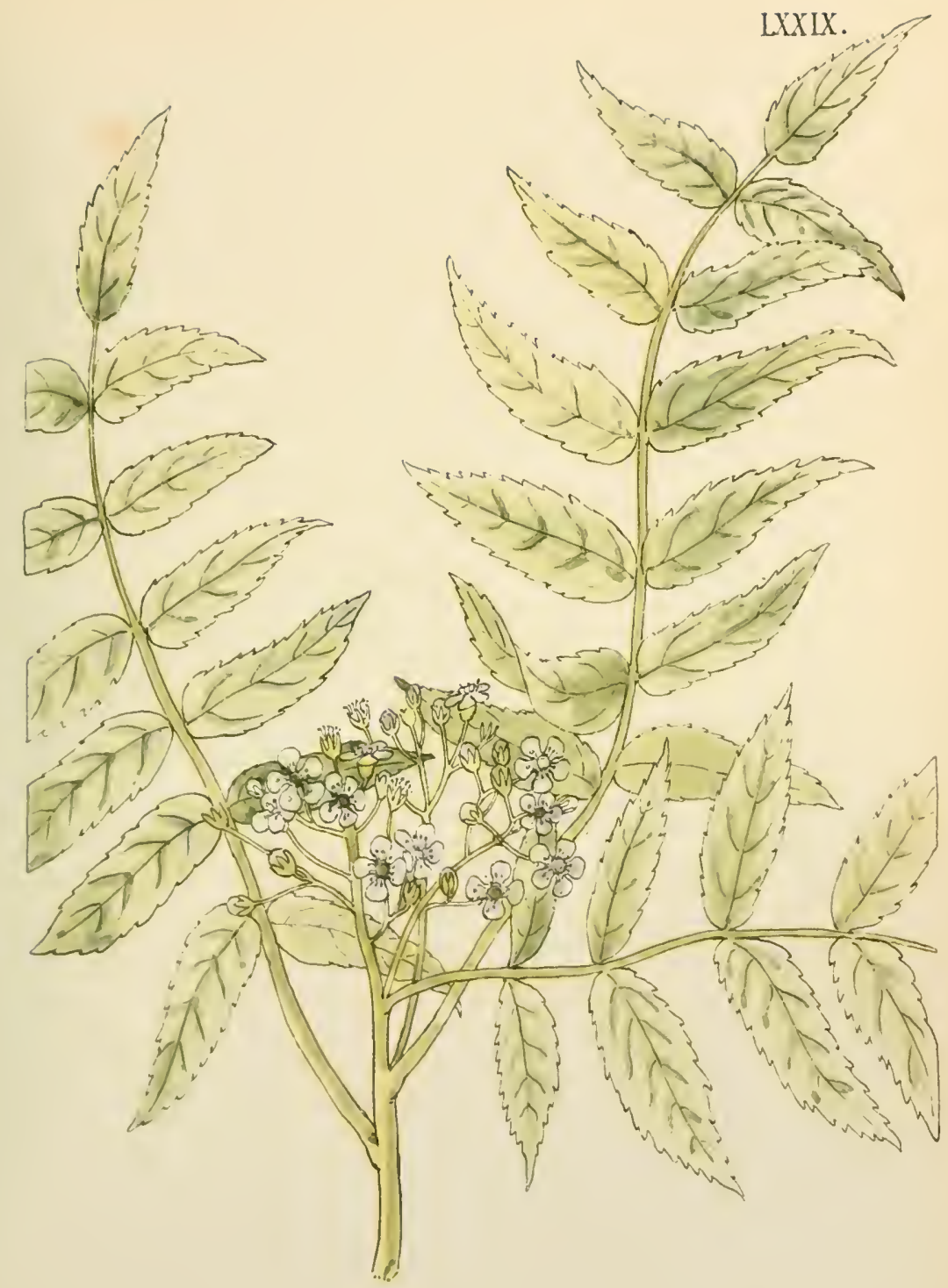

Pyrus aucuparia. 

LXXX.

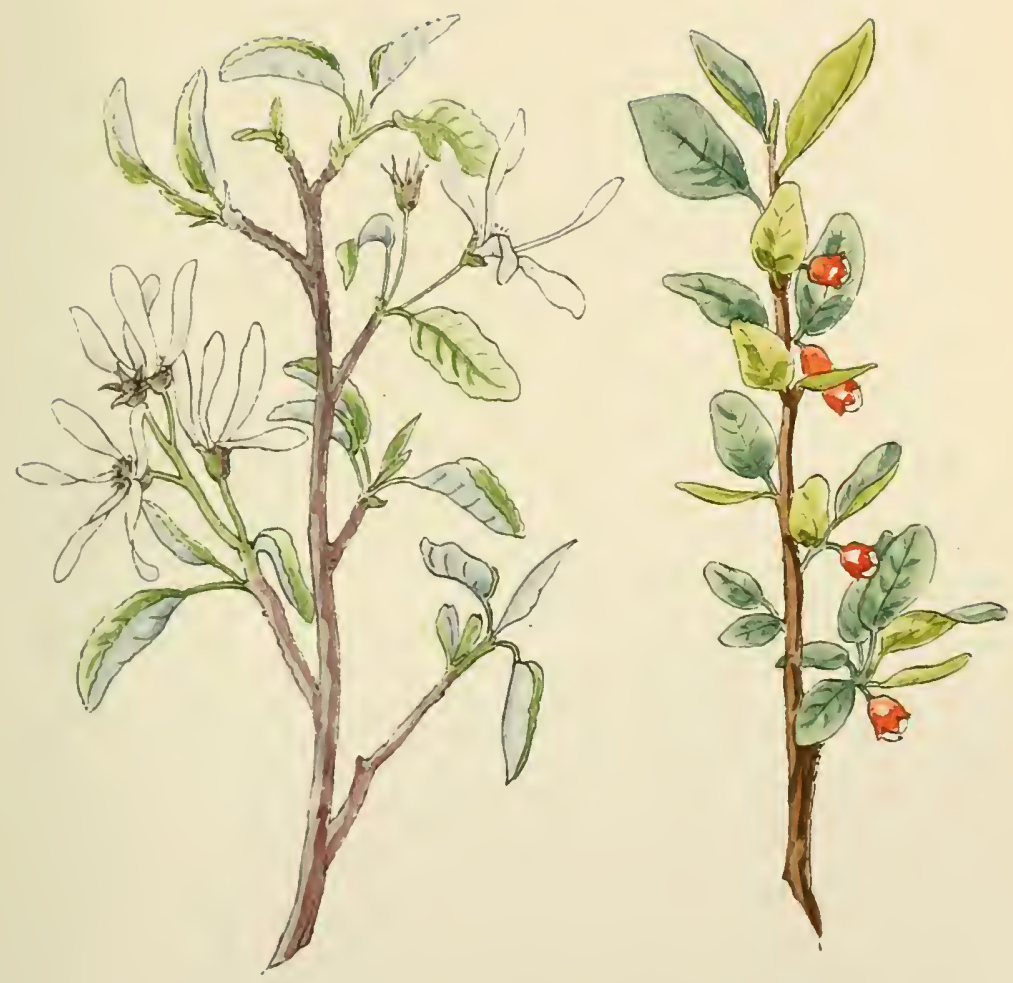

Amelanchier vulgaris. Cotoneaster vulgaris. 



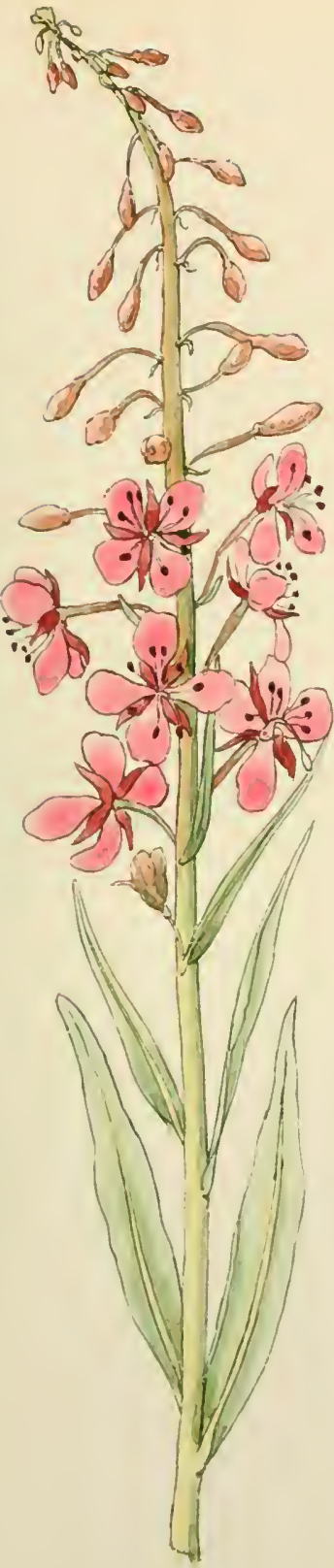

Epilobium spicatum.

\section{LXXXI.}

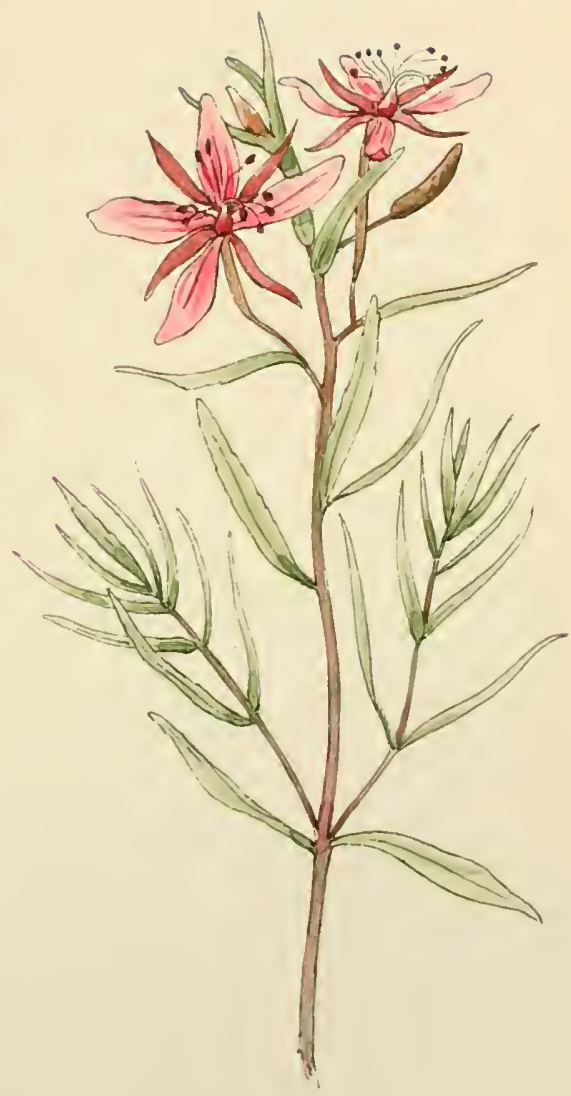

Epilobium Fleischeri. 

LXXXII.

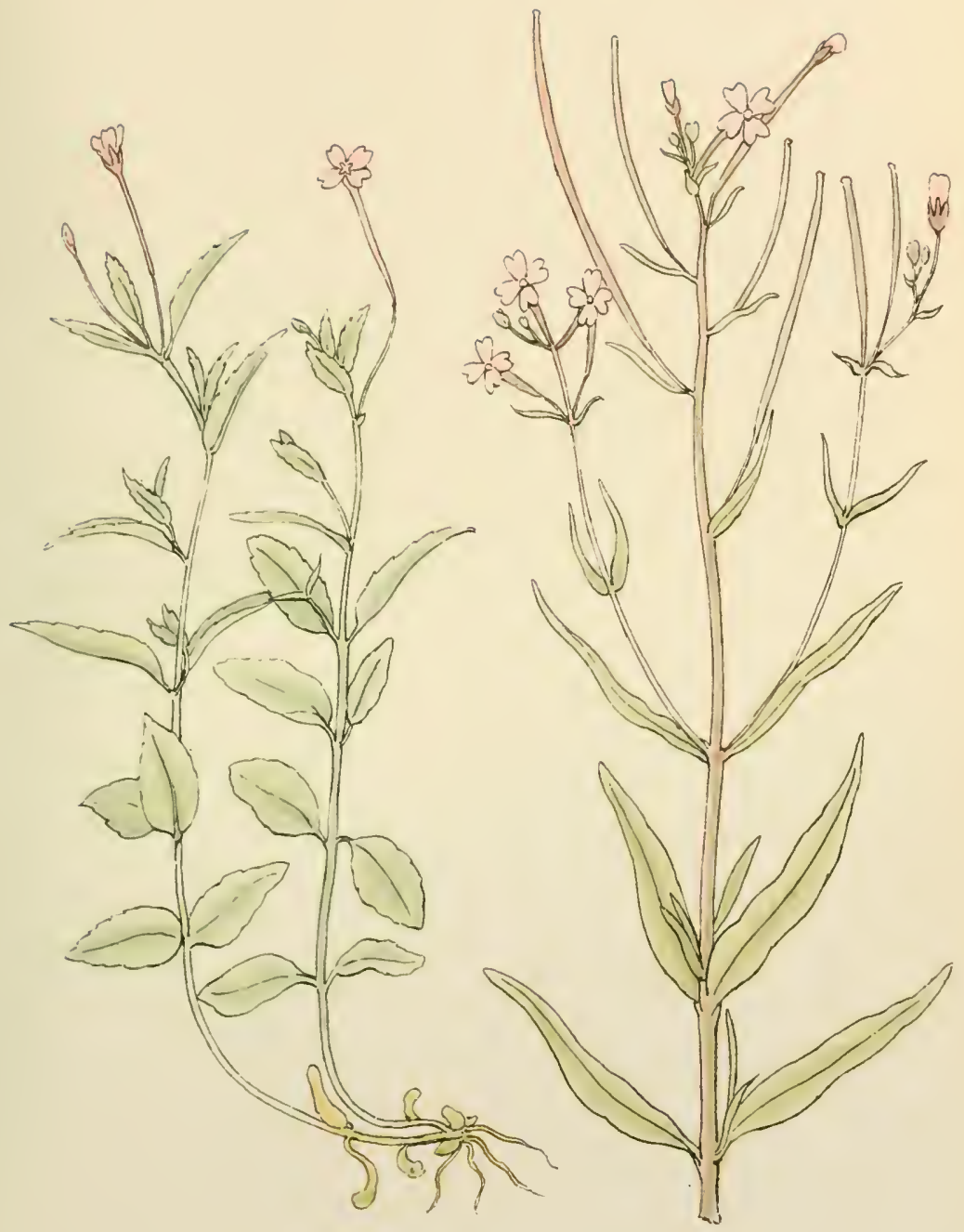

Epilobium montanum.

Epilobium palustre. 

LXXX11I.
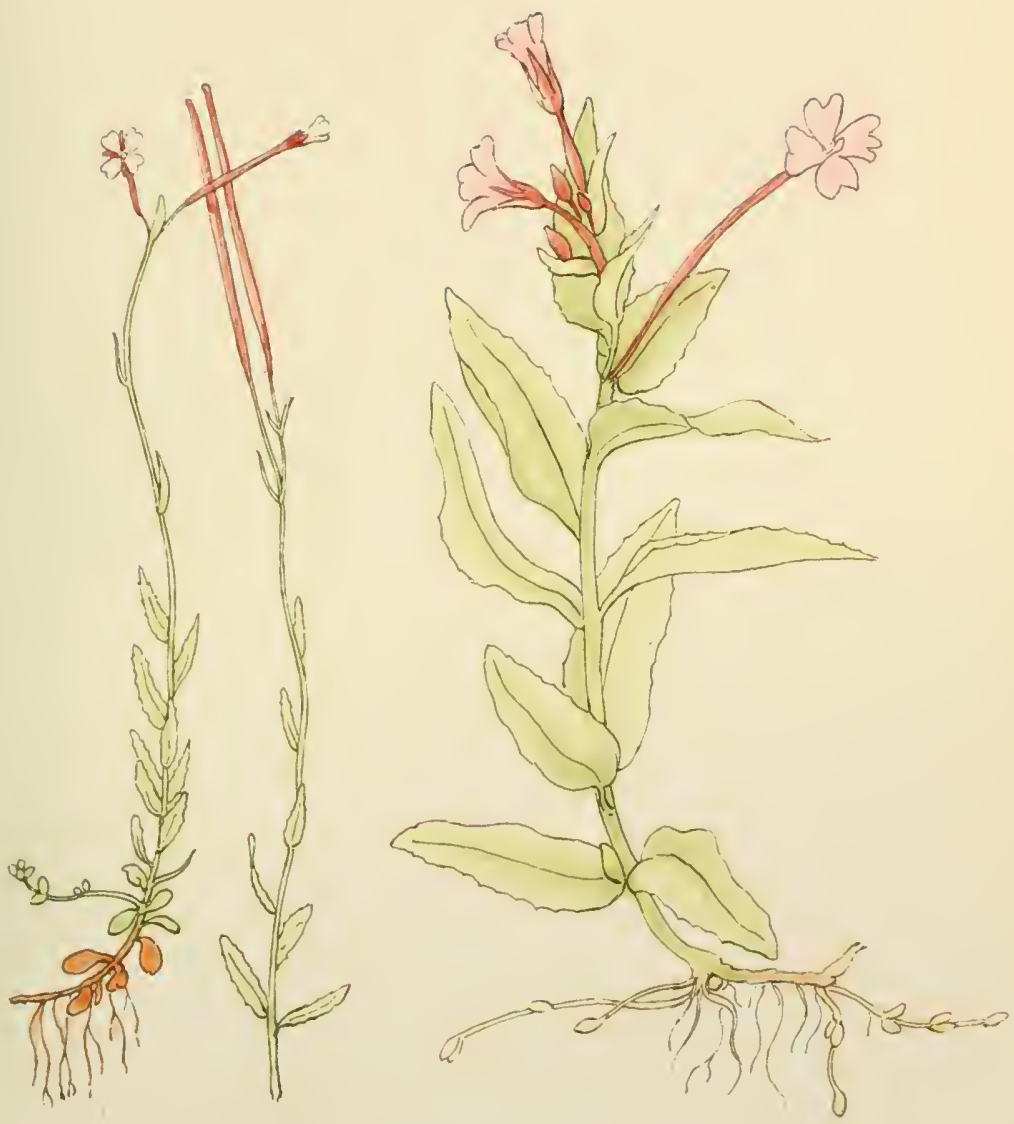

Epilobium alpinum .

Epilobium origanifolium. 


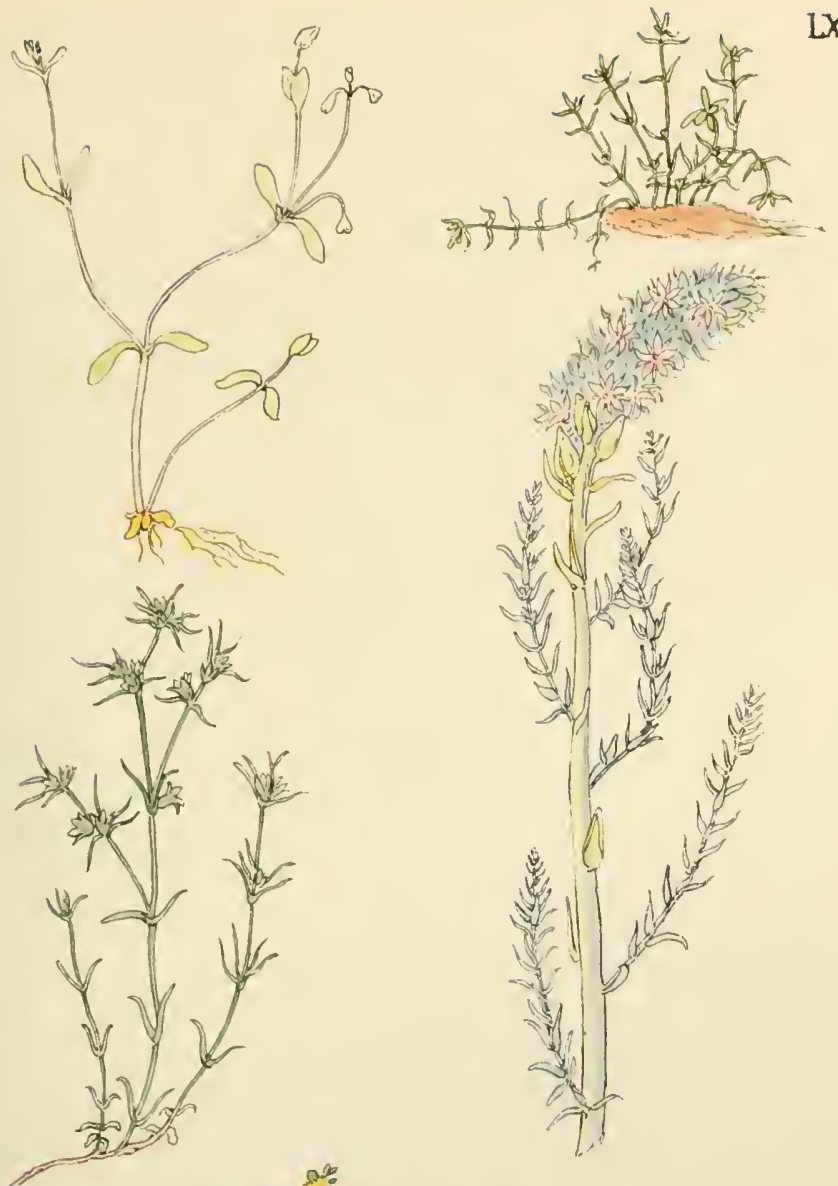

LXXXIV.
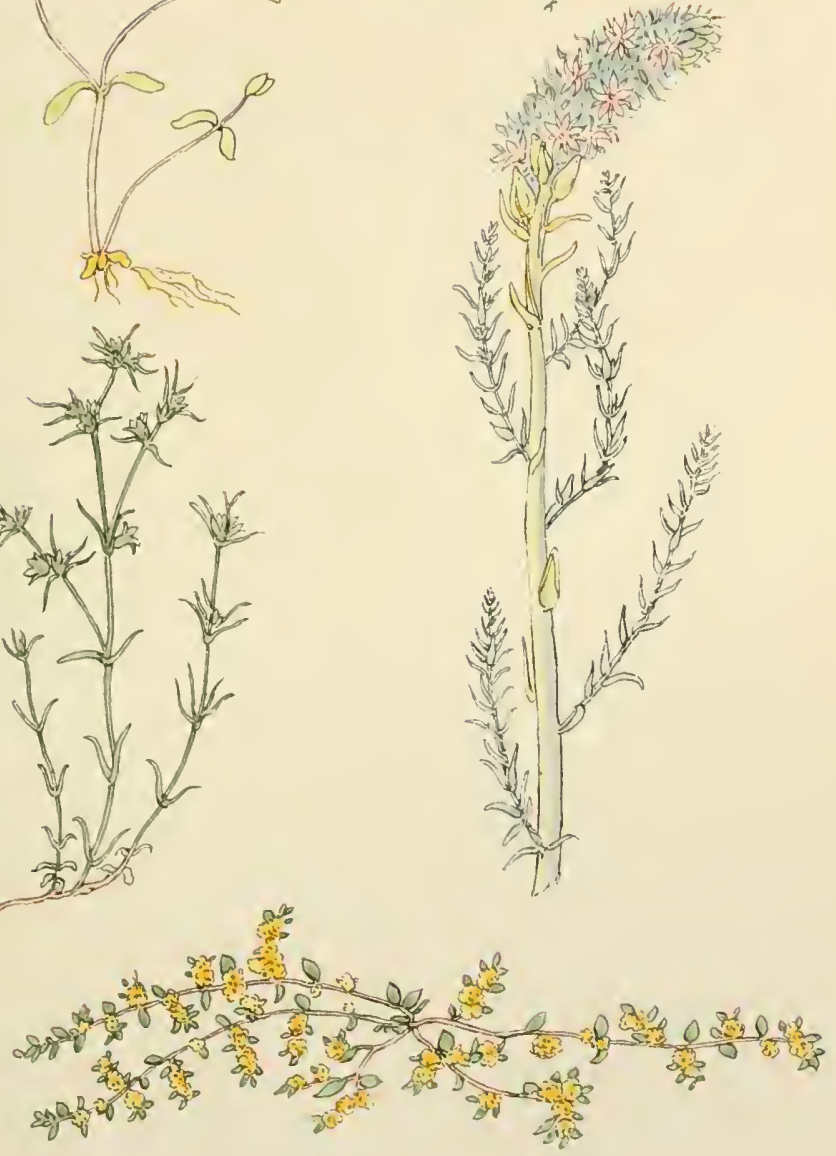

Montia fontana var. rivularis.

Callitriche stagnalis var. verna.

Scleranthus annuus.

Myricaria germanica.

Herniaria glabra. 


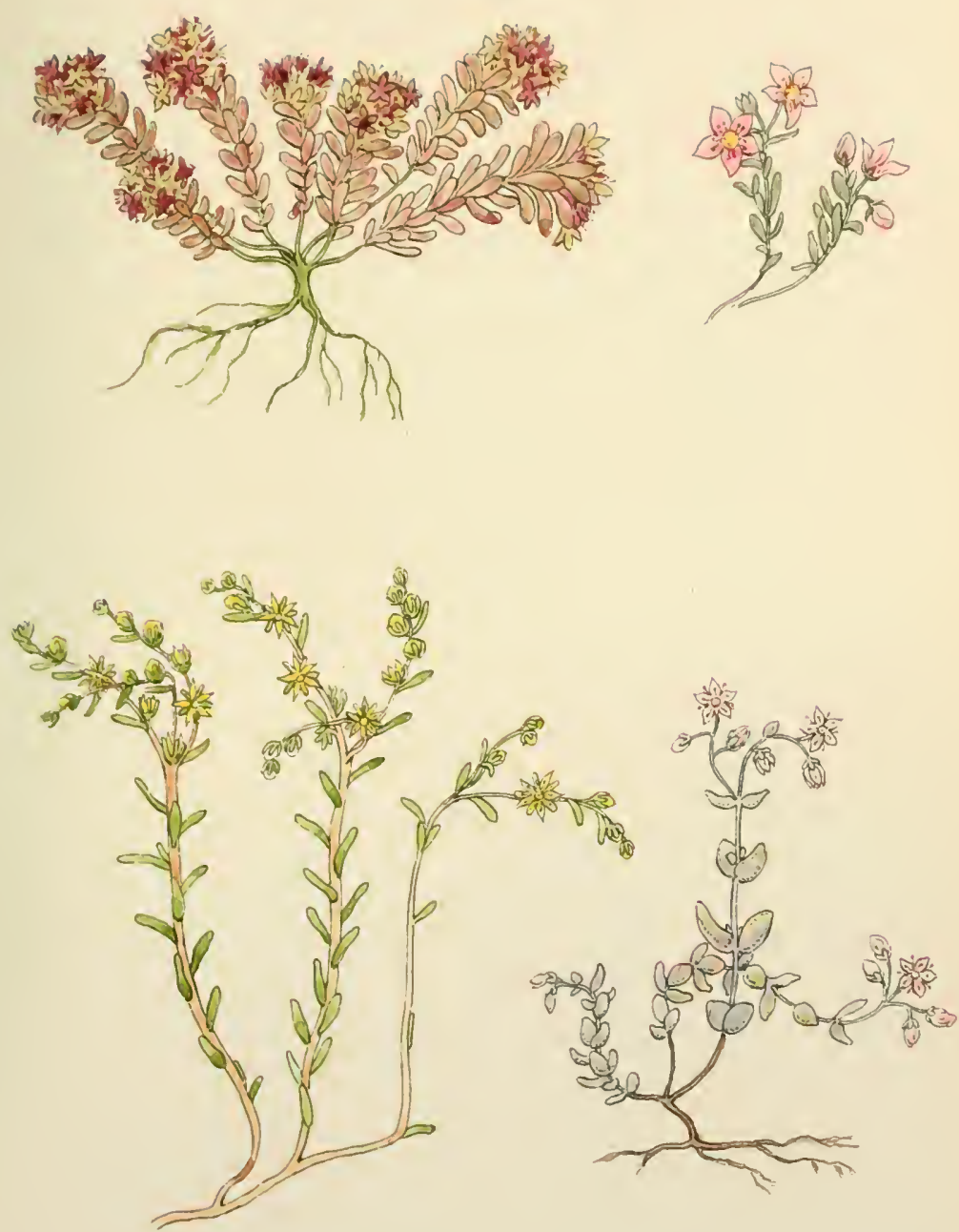

Sedum atratum.

Sedum villosum.

Sedum annuum.

Sedum dasyphyllum. 

LXXXVI.

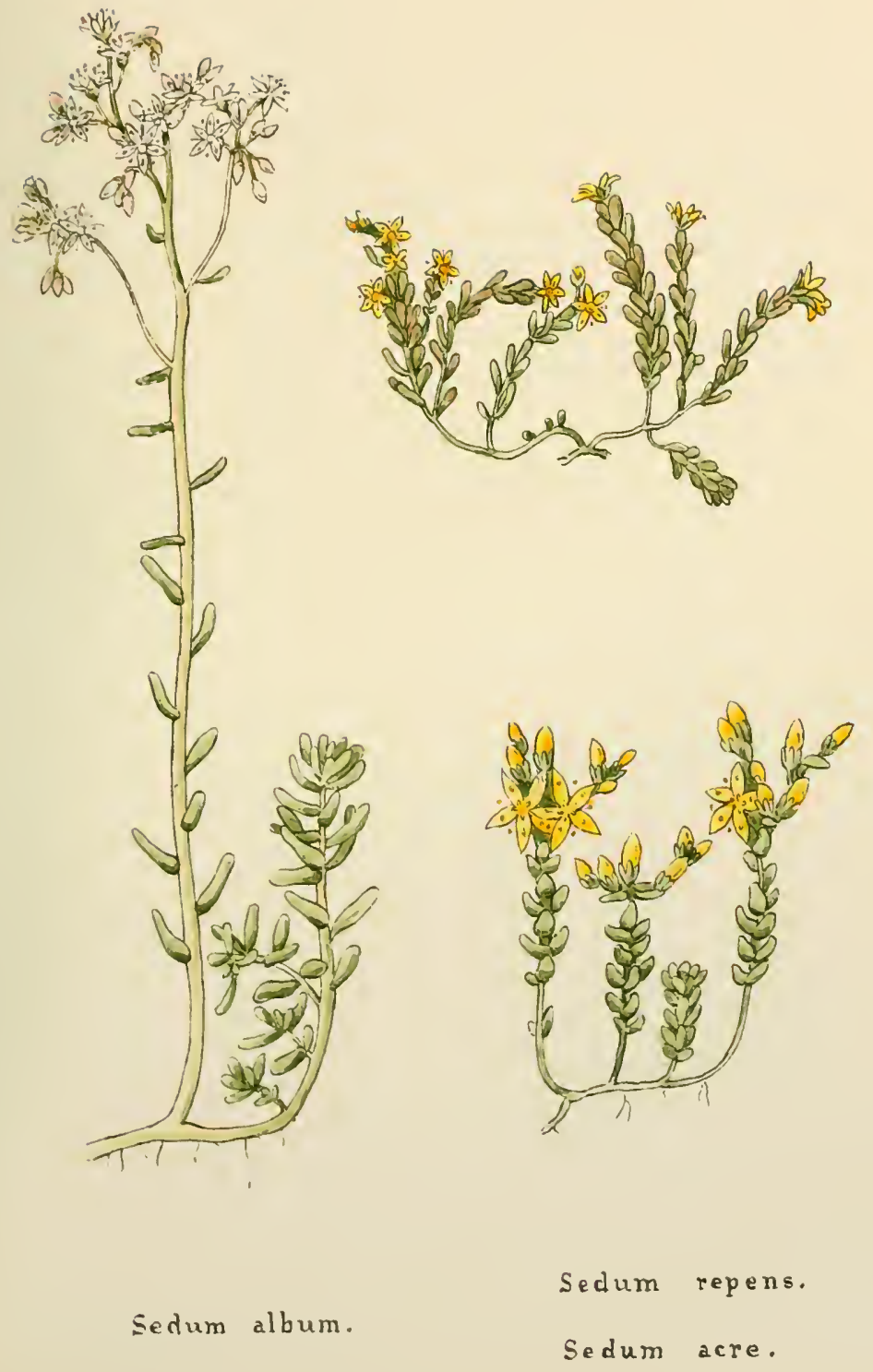




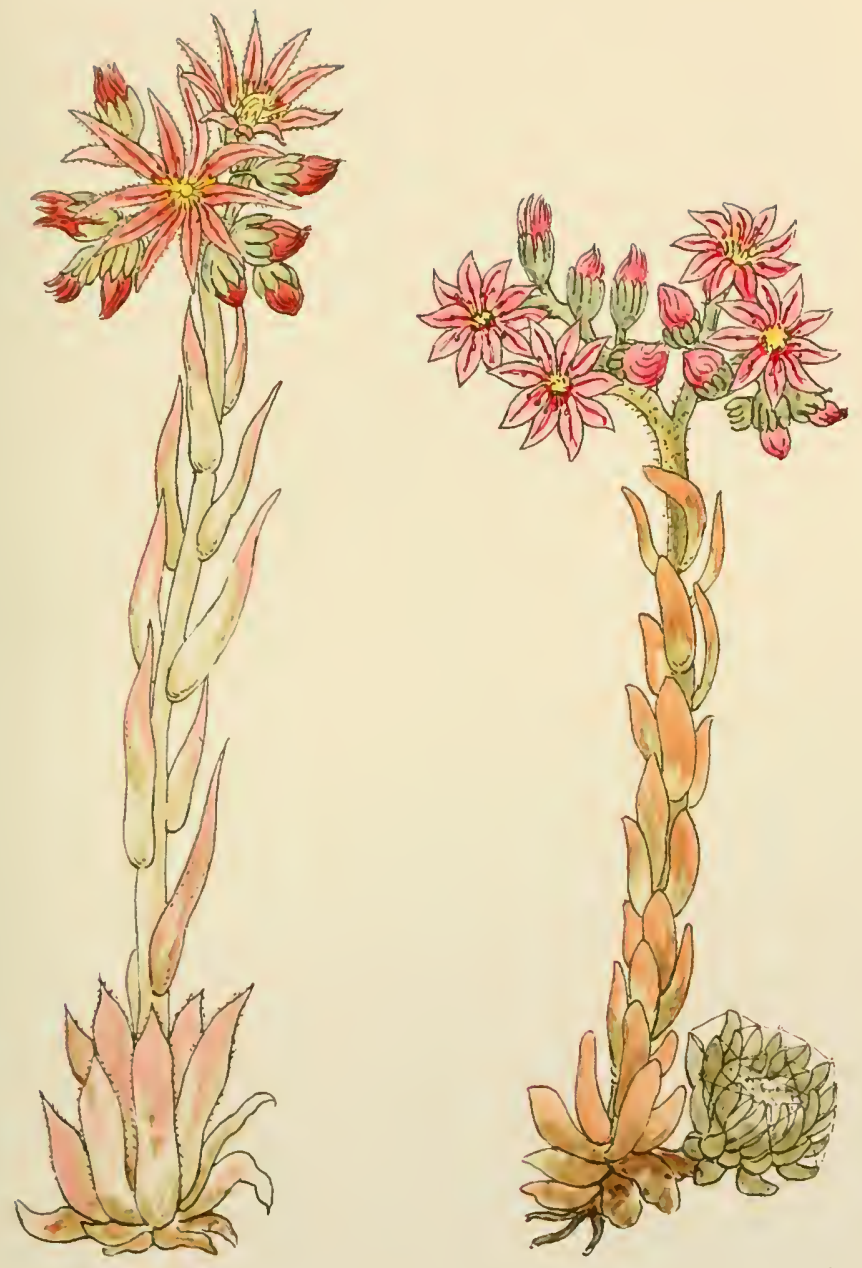

LXXXVIII .

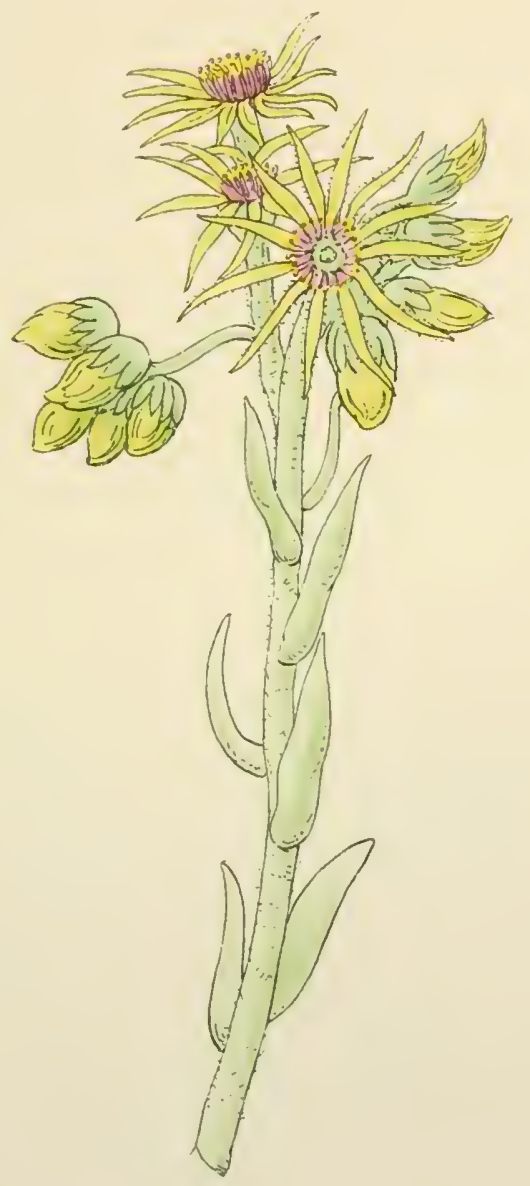

Sempervivum Wulfeni. 


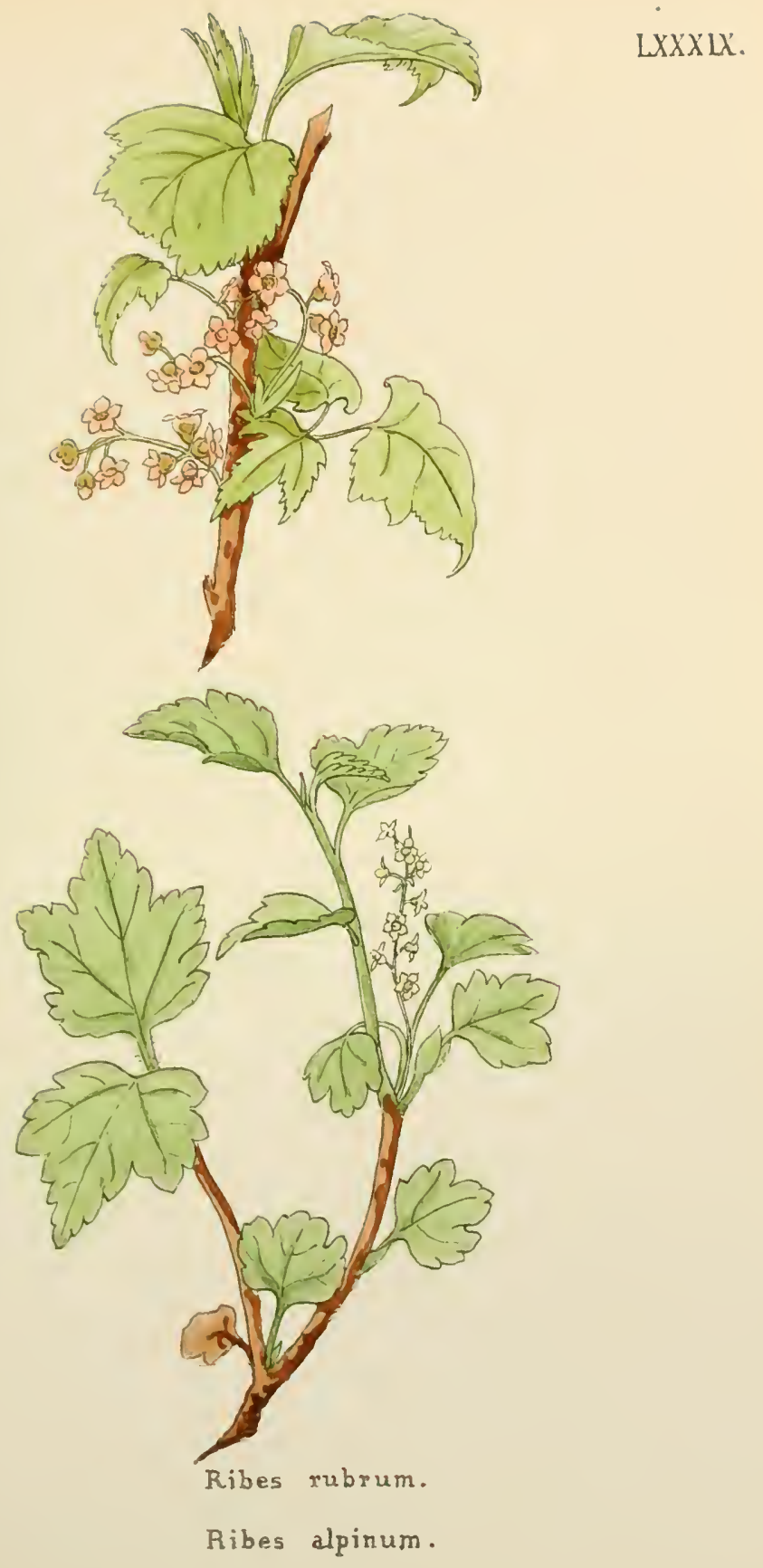



XC.
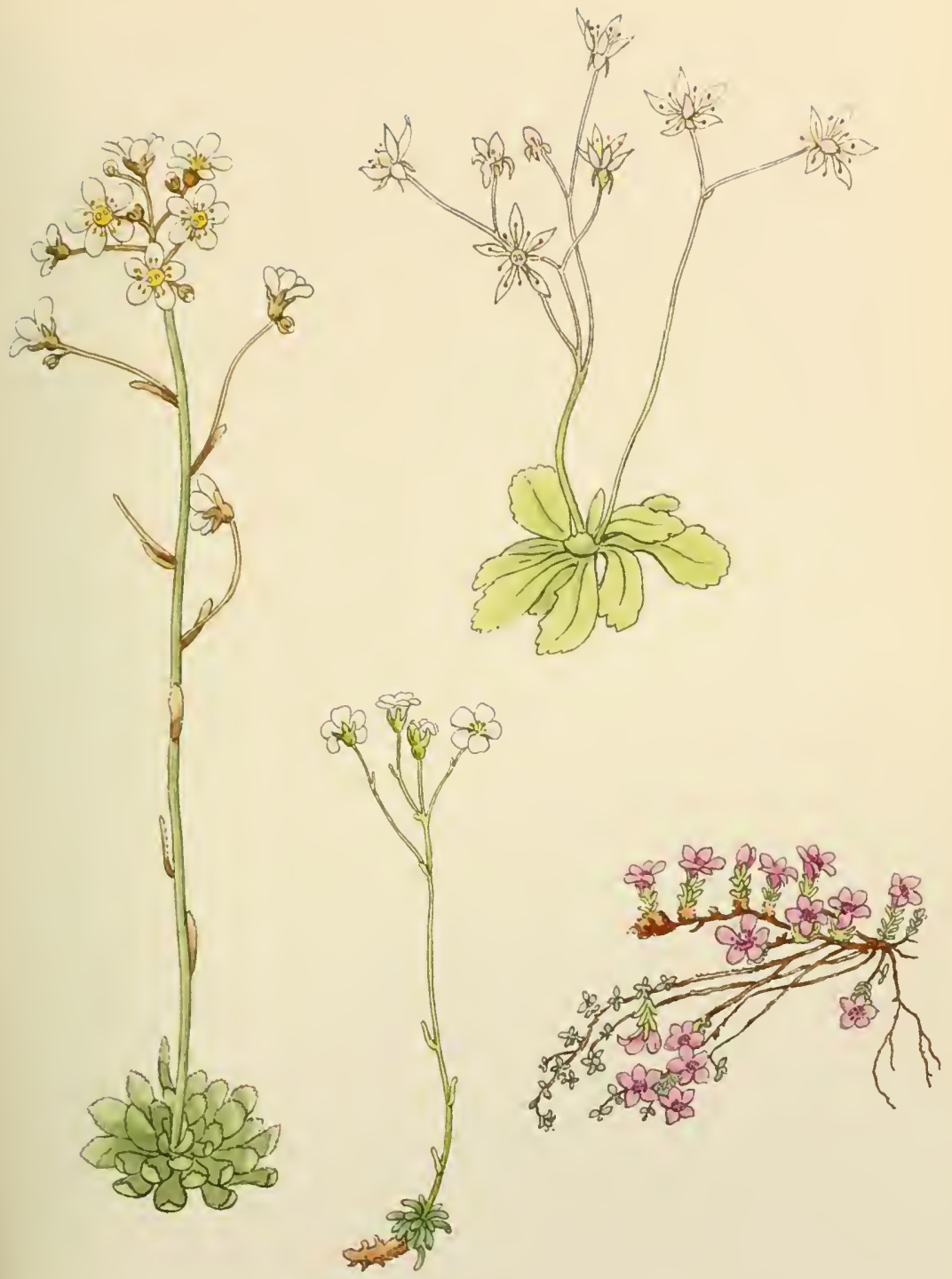

Saxifraga stellaris.

Saxifraga Aizoon. Saxifraga casia. Saxifraga oppositifolia. 

XCI.
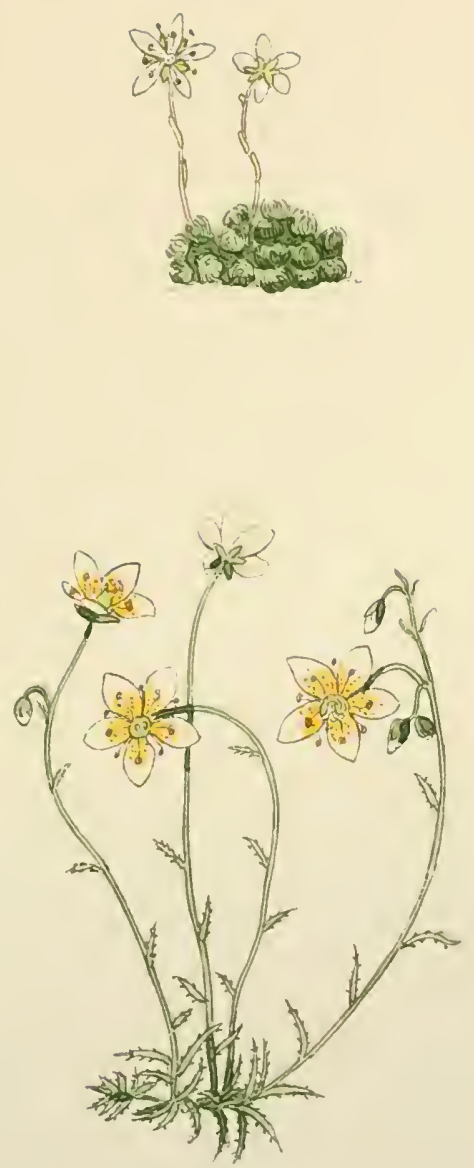

Saxifraga bryoïdes.

Sarifraga aspera. 



\section{XCII.}
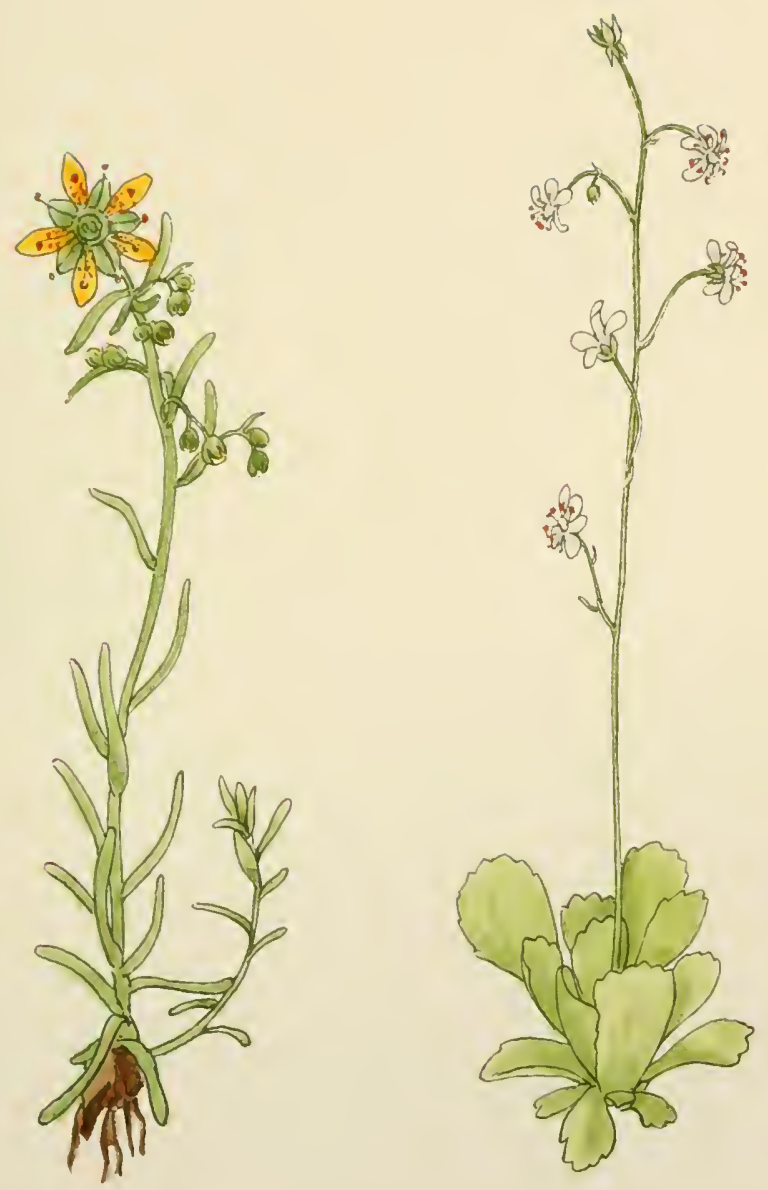

Saxifraga aizoïdes.

Saxifraga cuneifolia. 

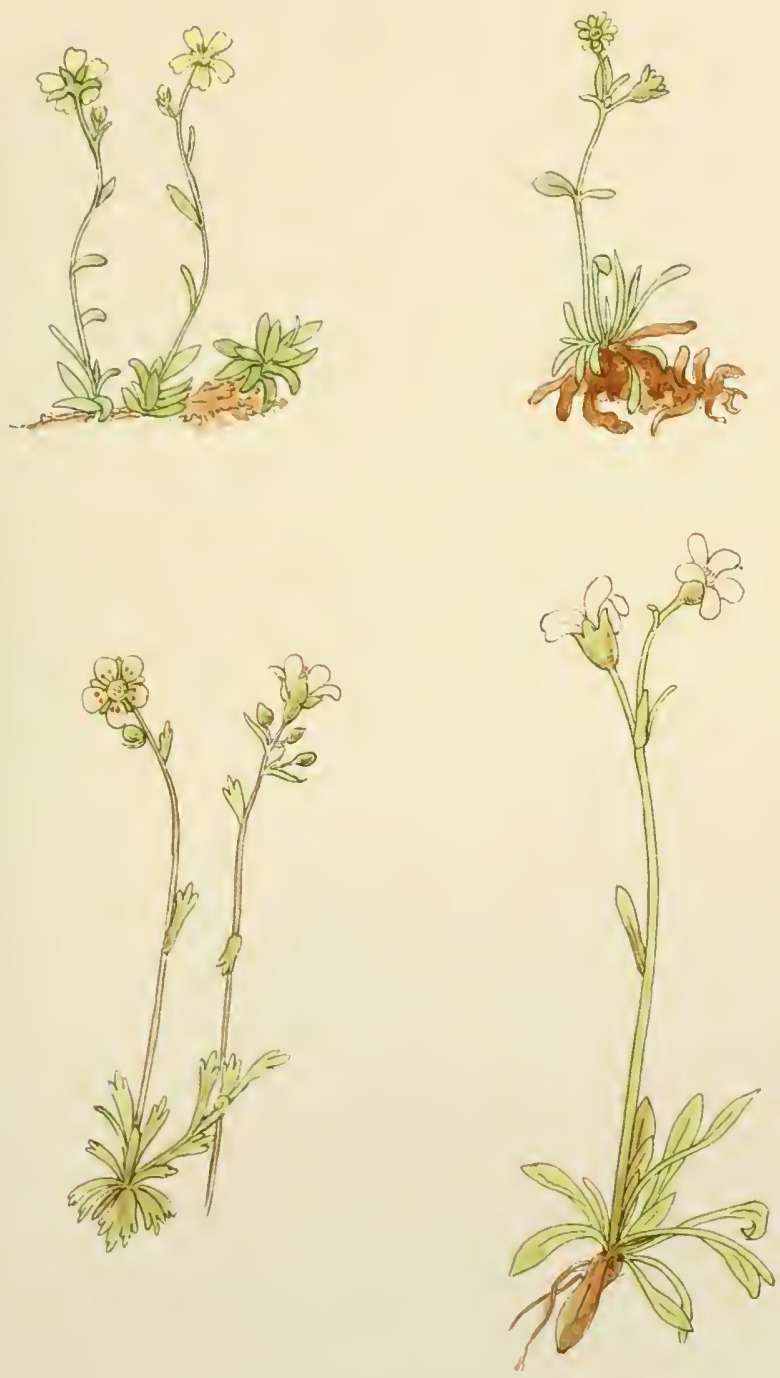

Saxifraga planifolia. Saxifraga Saguieri. Saxifraga exarata. Saxifraga androsacea. 


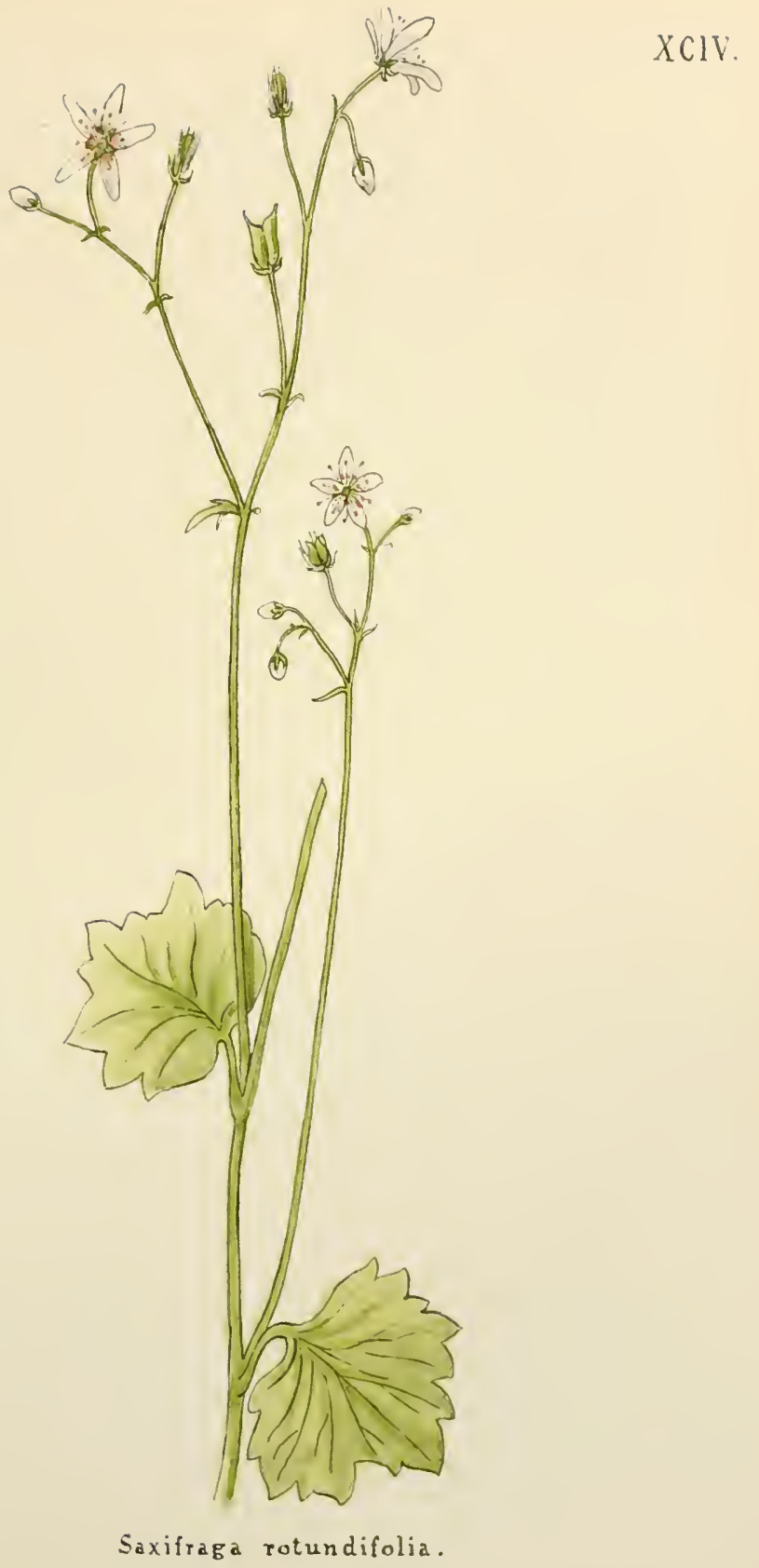




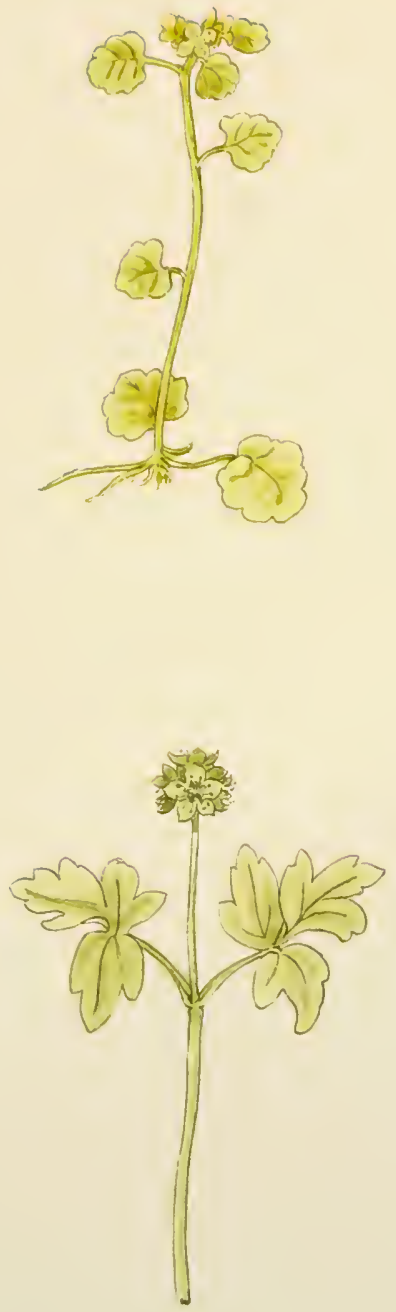

Chrysosplenium alternifolium .

Adoxa Moschatellira. 



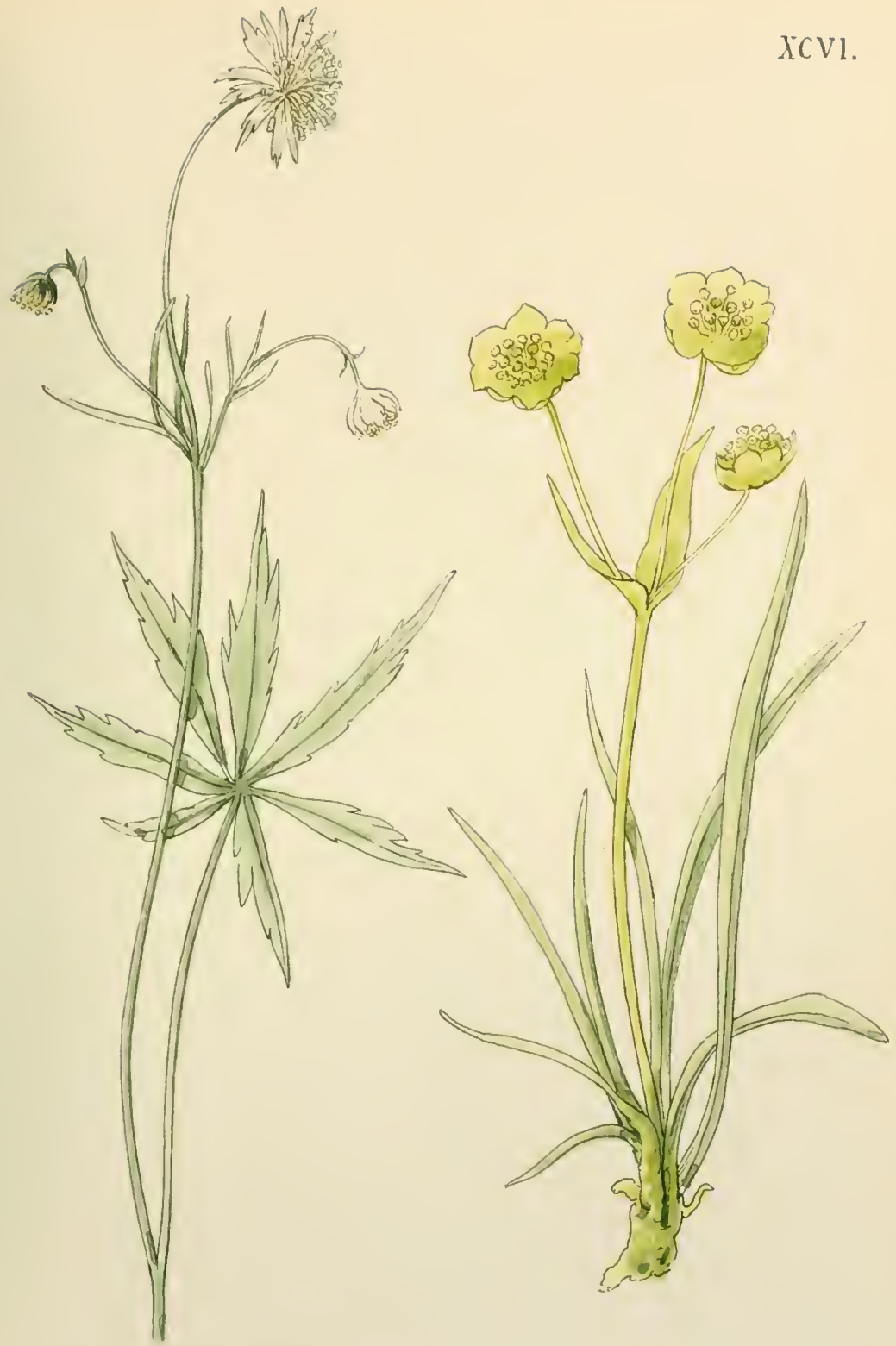

Astrantia minor.

Bupleurum stellatum . 

XCVII.

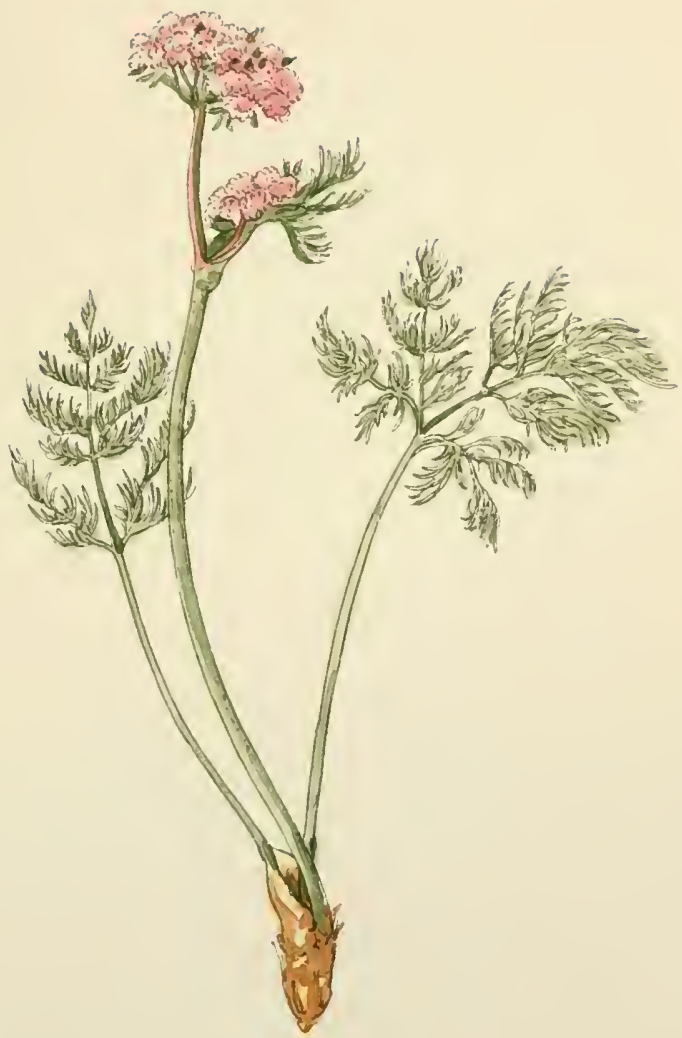

Meum Mutellina. 



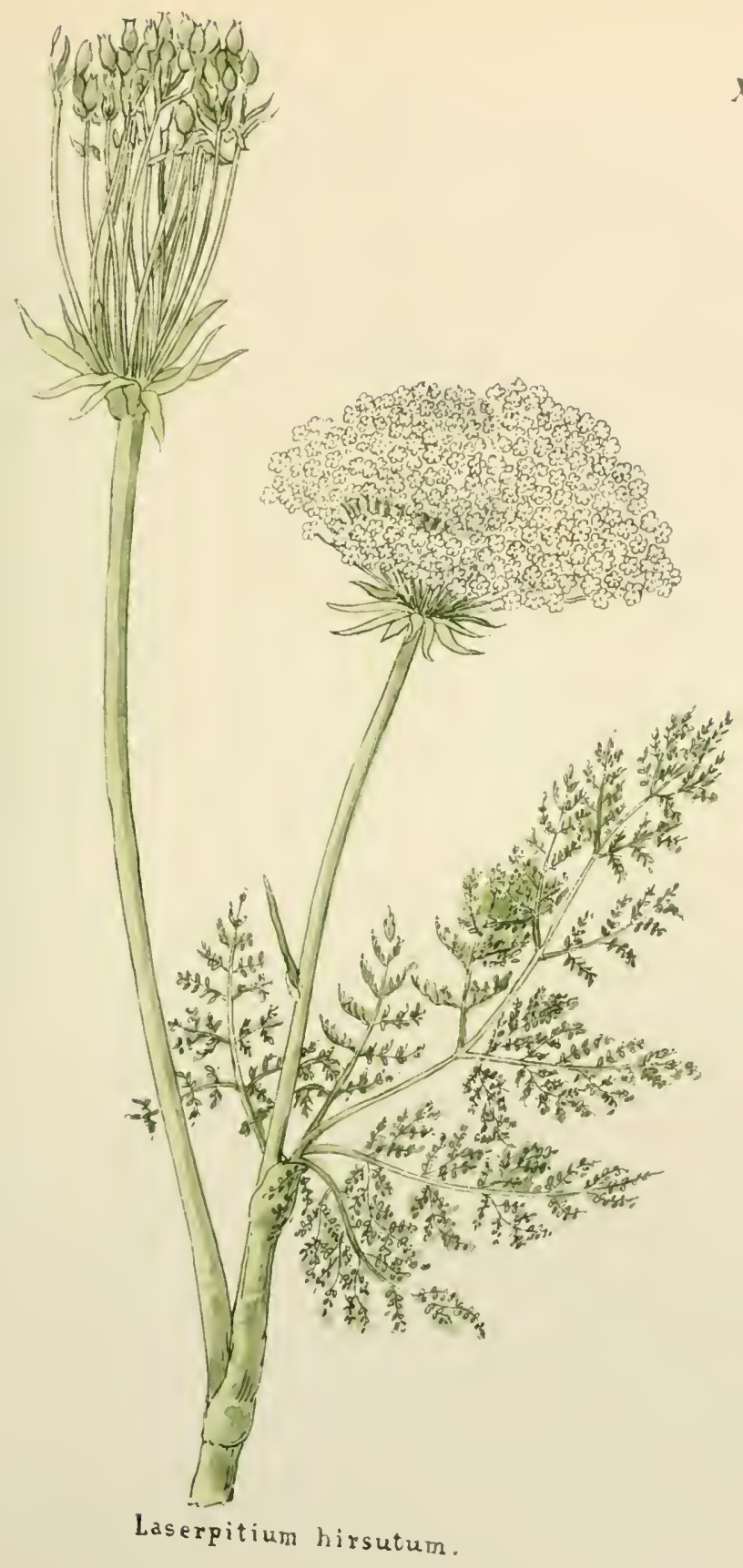



XCIX.

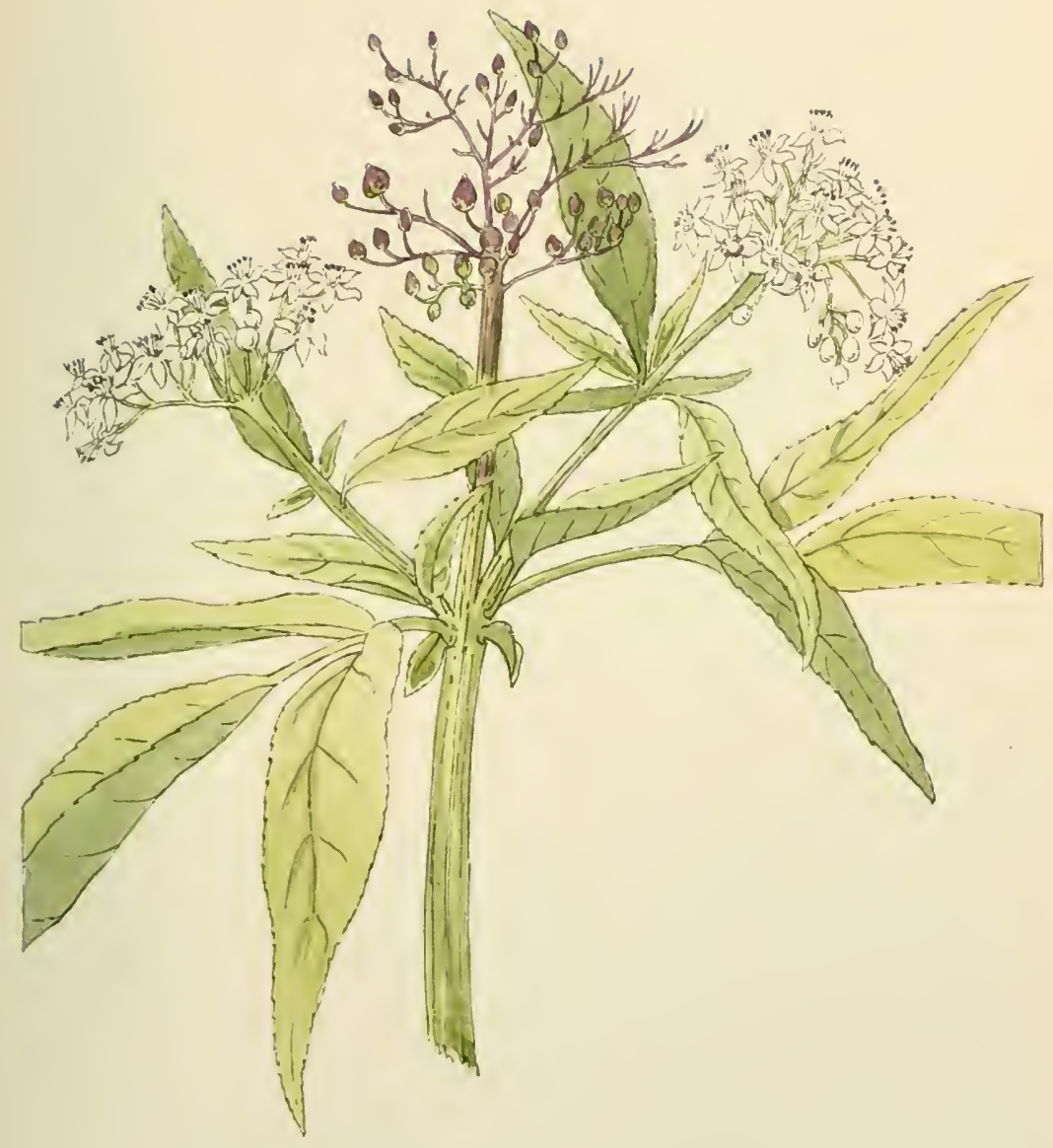

Sambucus Ebulus. 



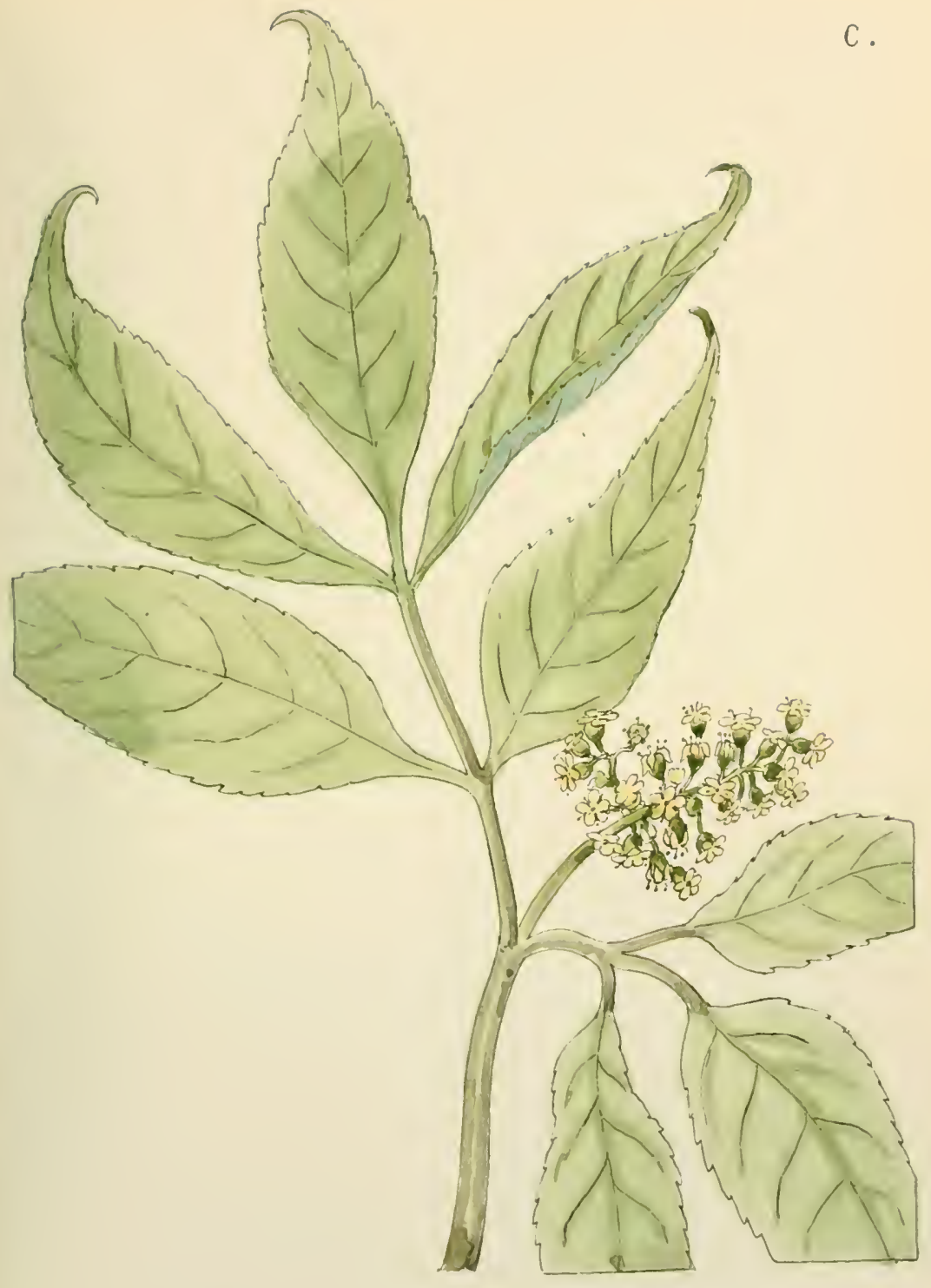





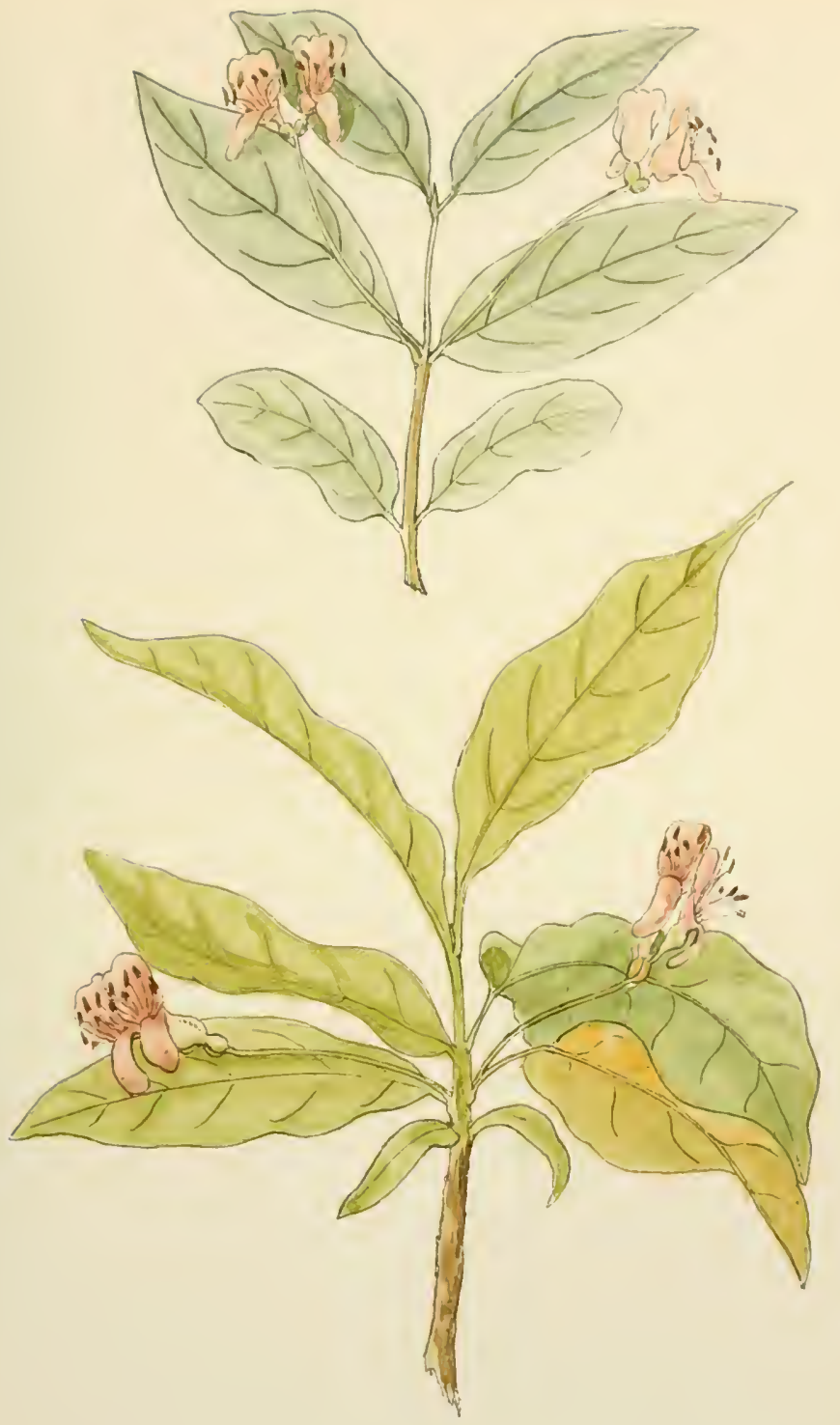

Cl. 



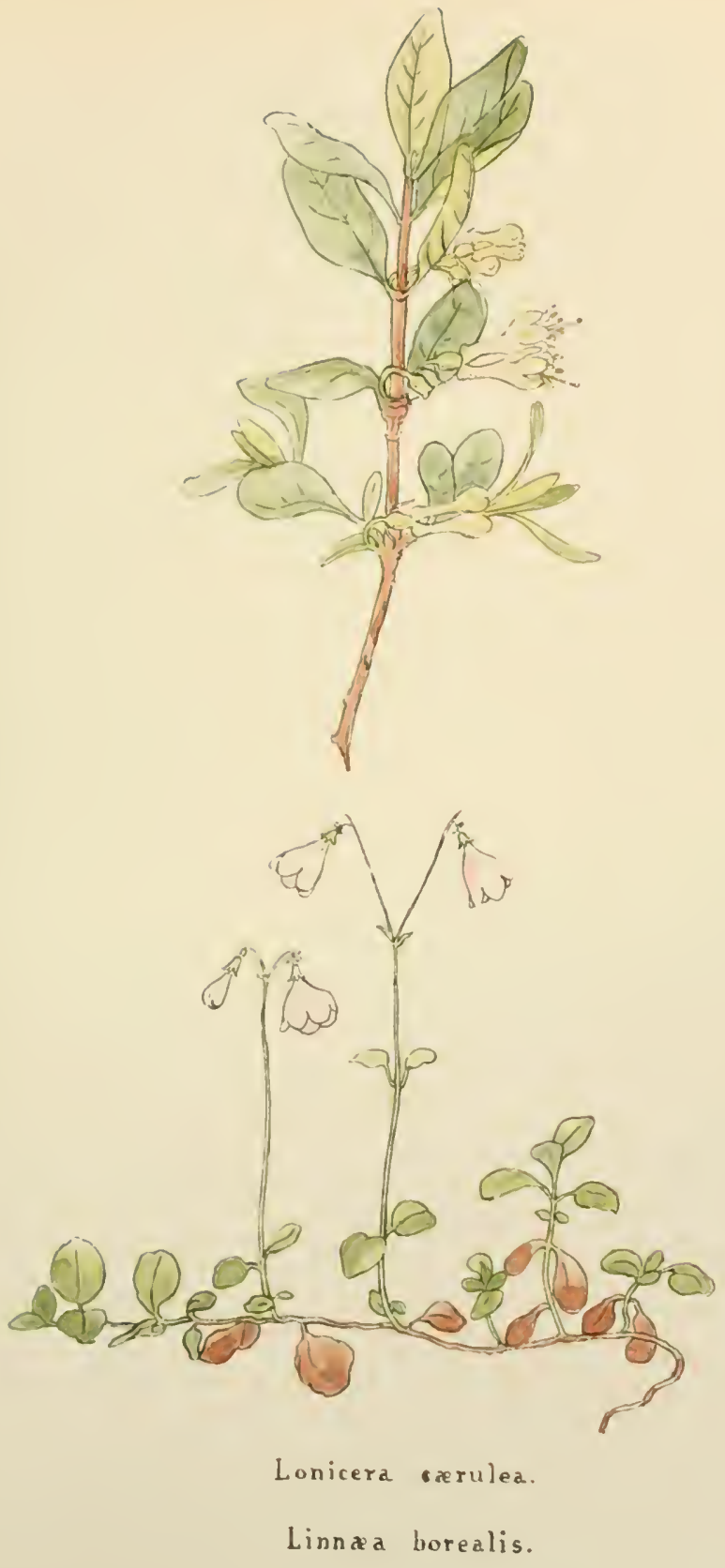

CII. 



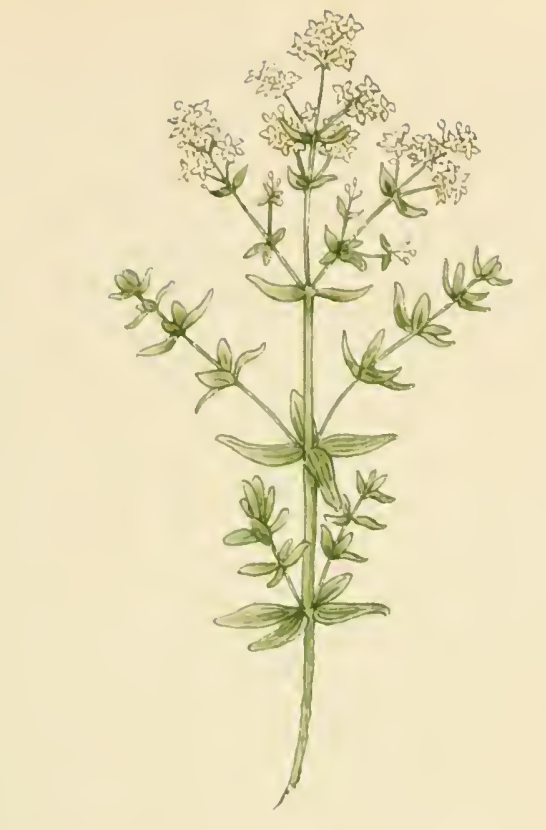

CIII.
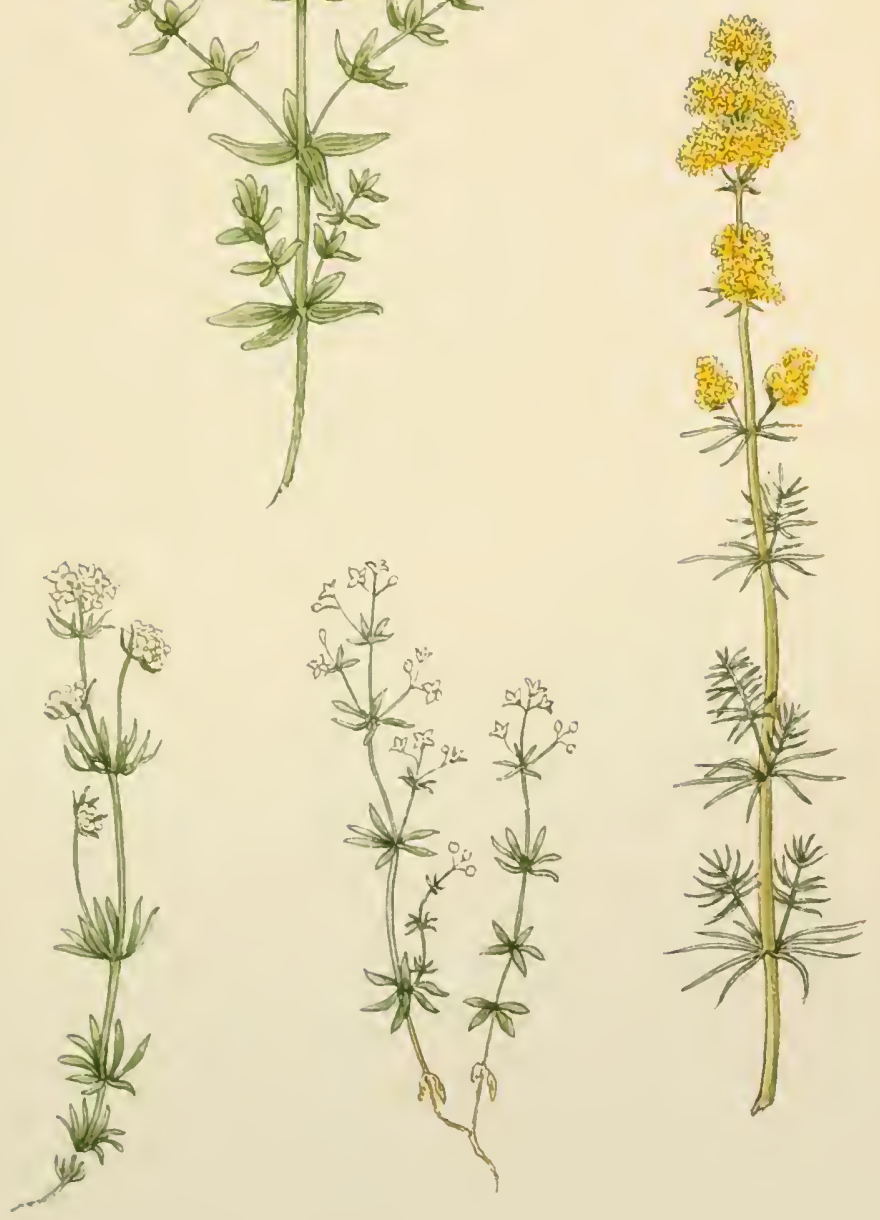

Galium boreale.

Galium sylvestre. Galium uliginosum. Galium verum. 



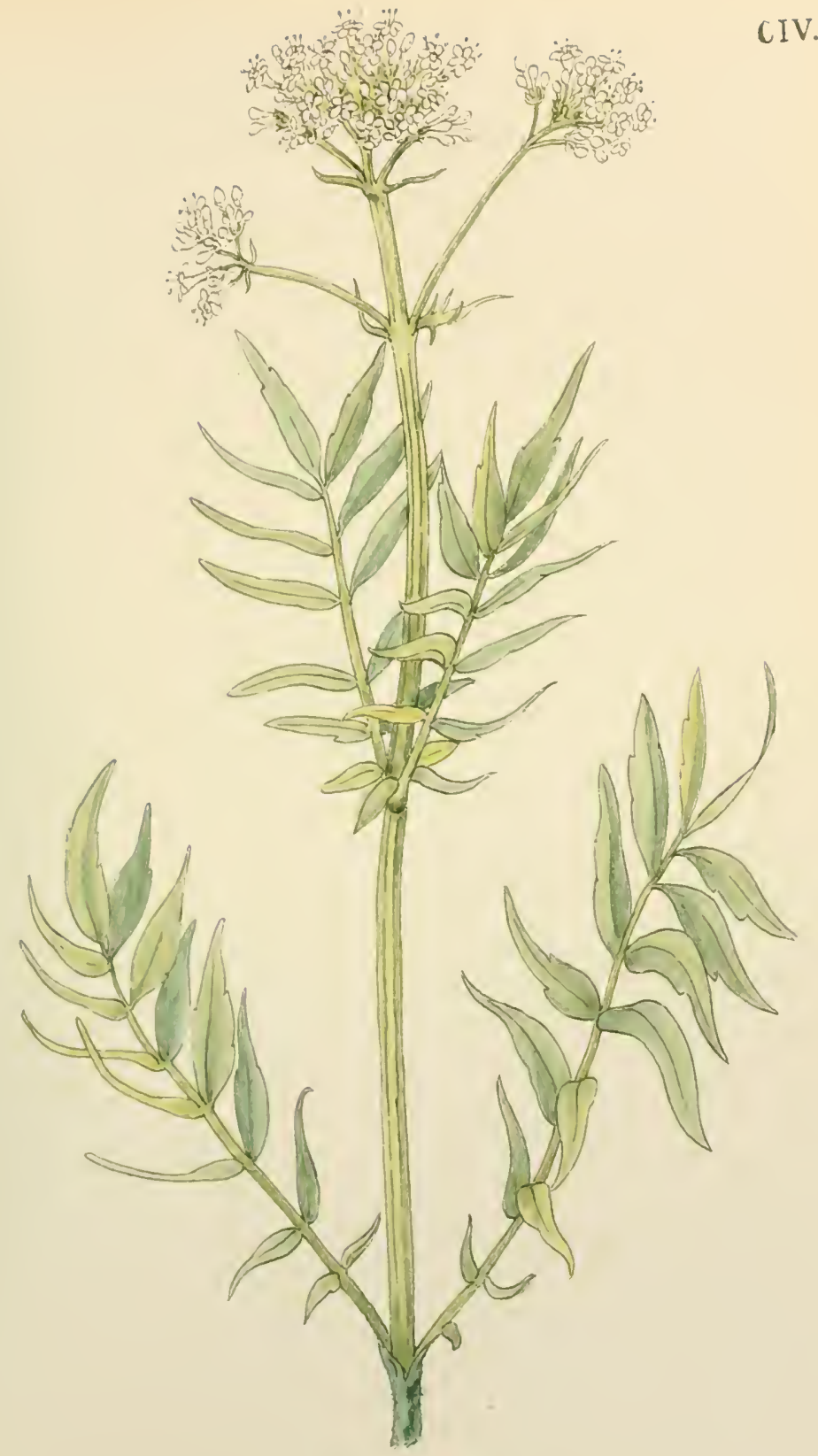

Valeriana officinalis. 



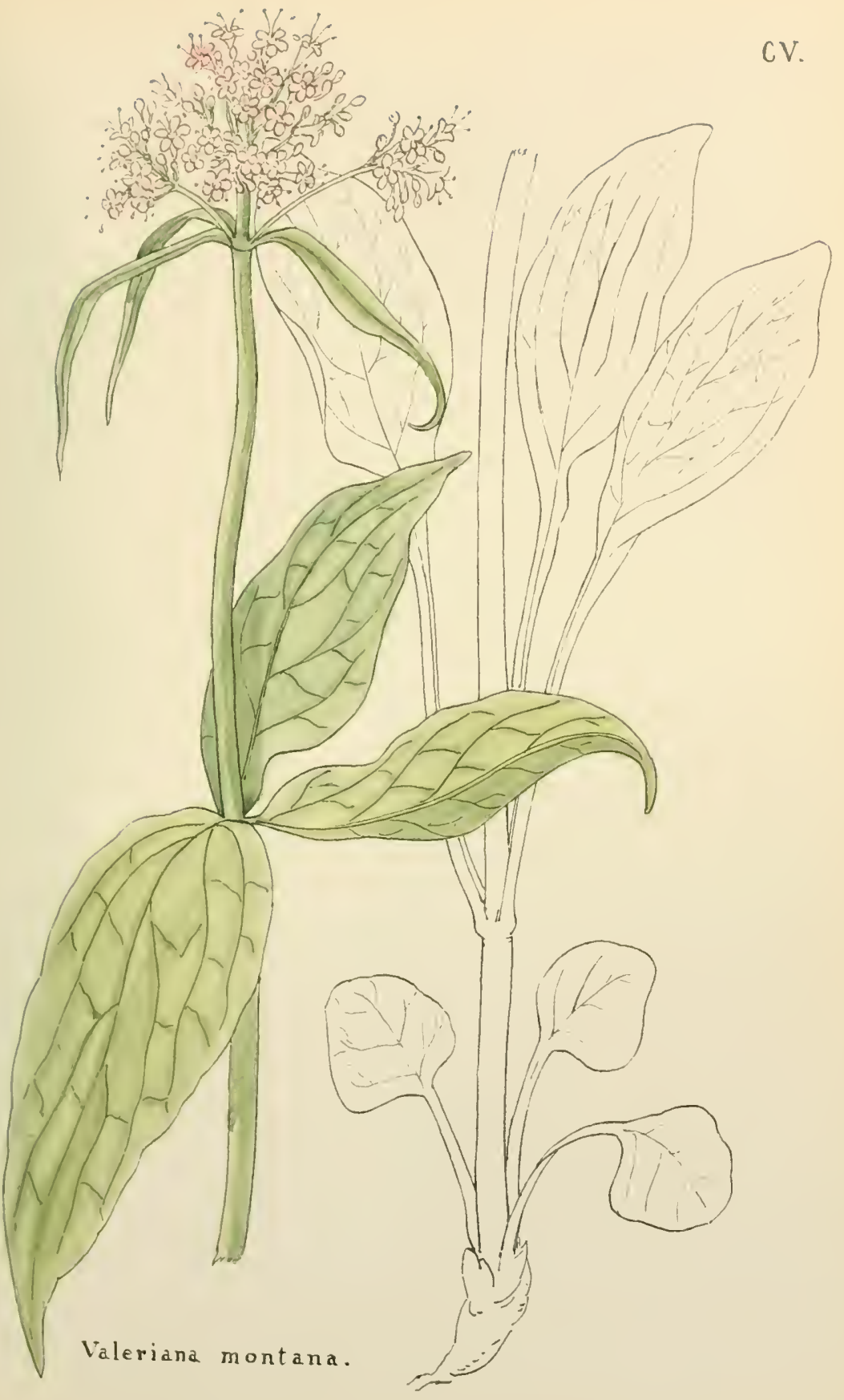



CVI.

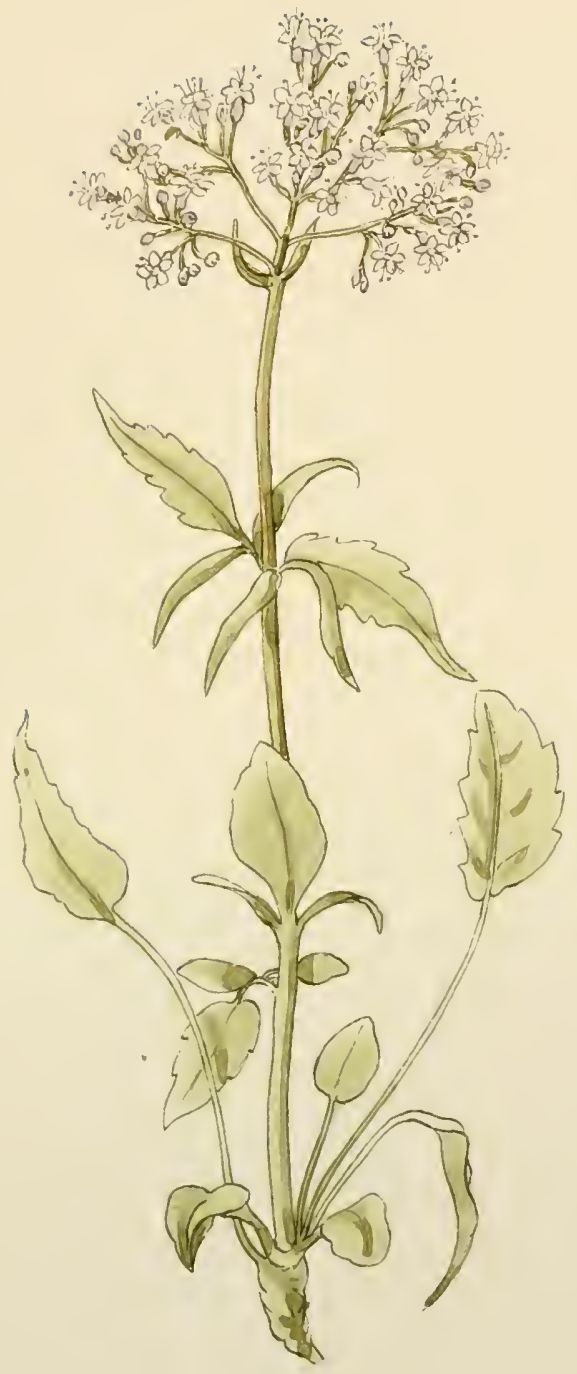

Valeriana tripteris. 



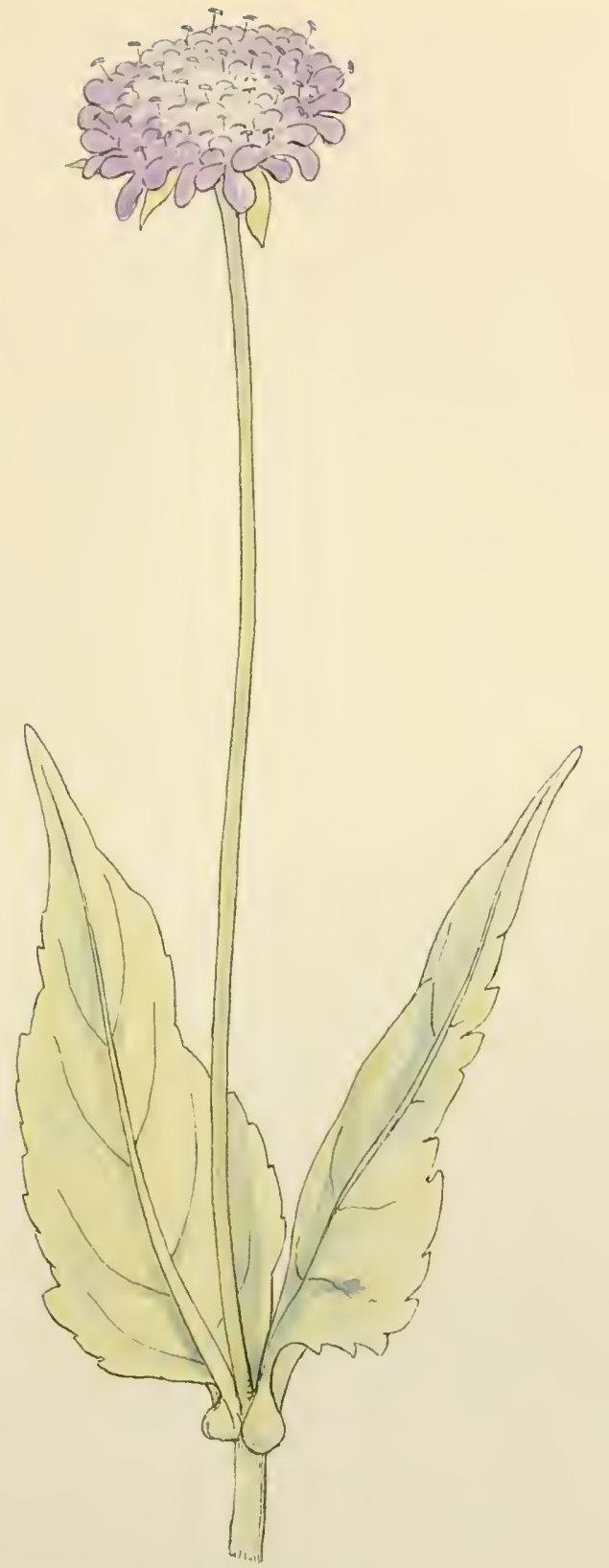

CVII.

Kinautia arvensis. 

CVIII.

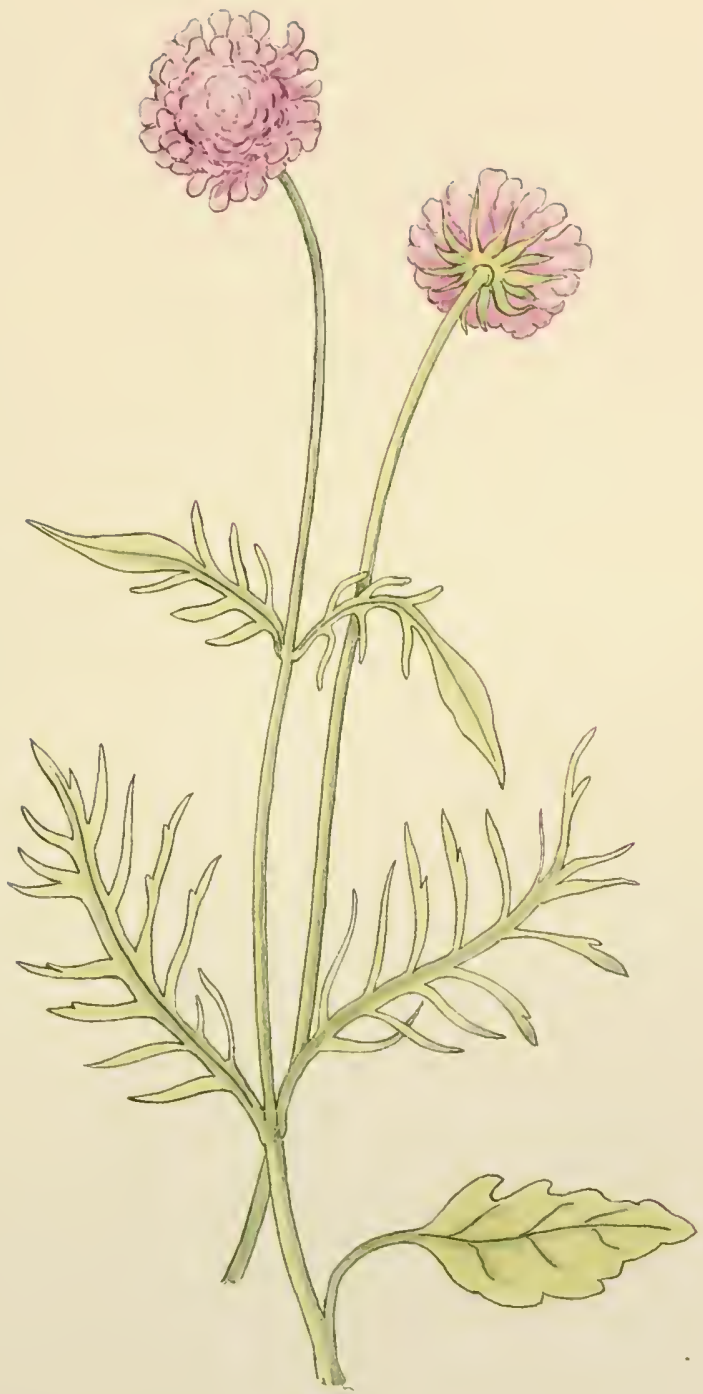

Scabiosa Columbaria var lucida. 
CIX.

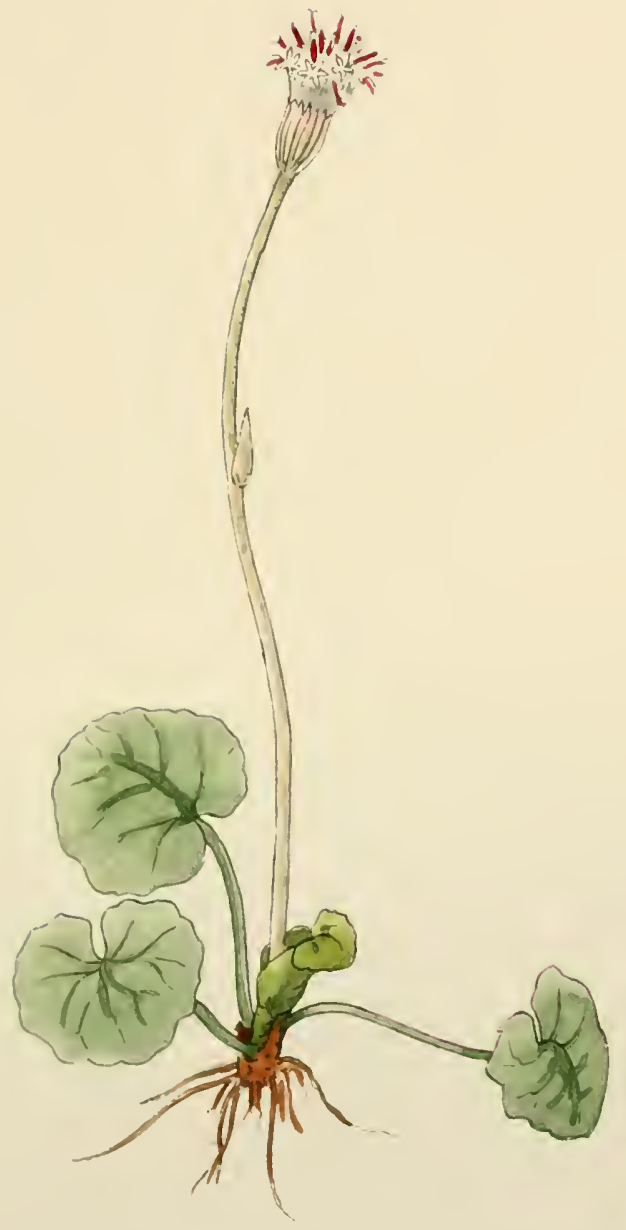

Homogyne alpina. 

CX.

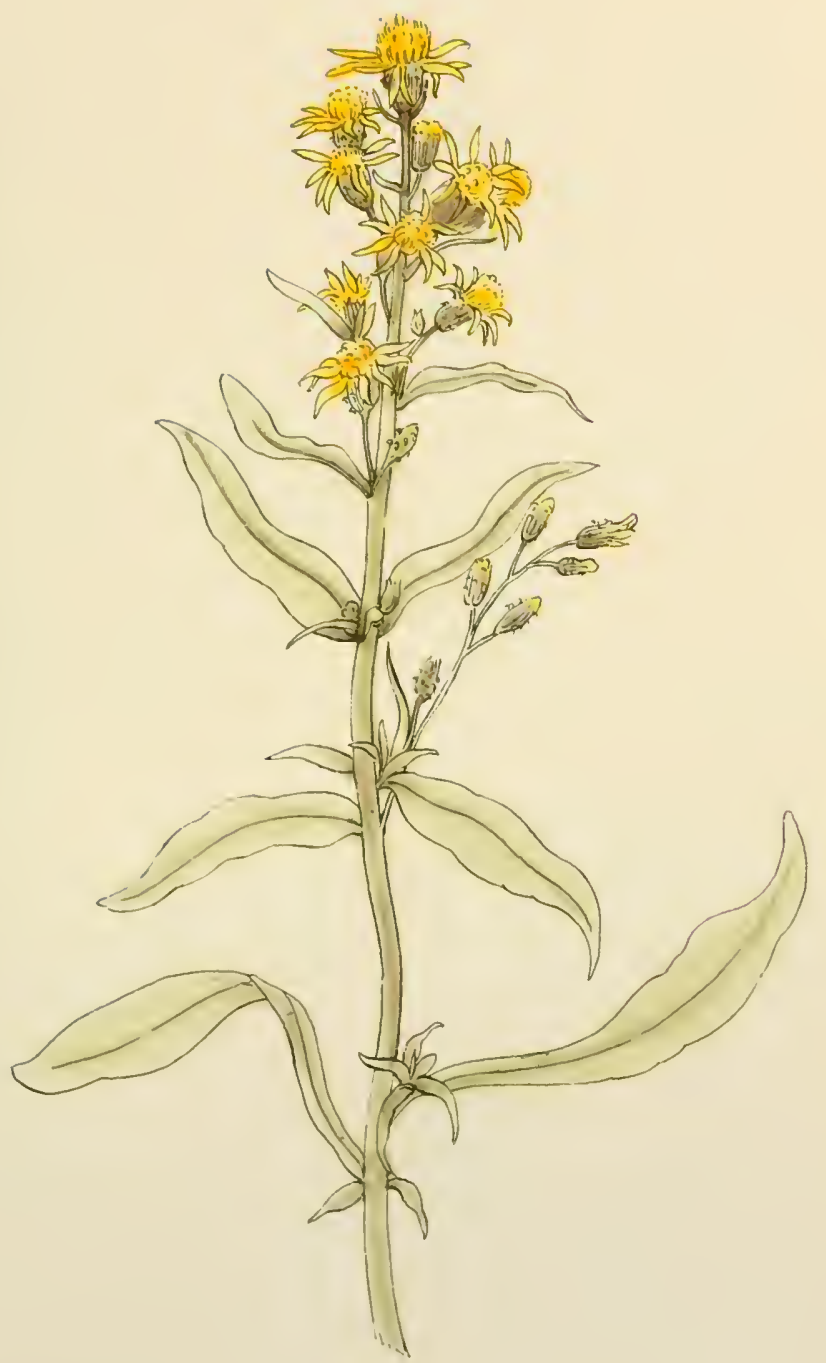

Solidago Virga-aurea. 



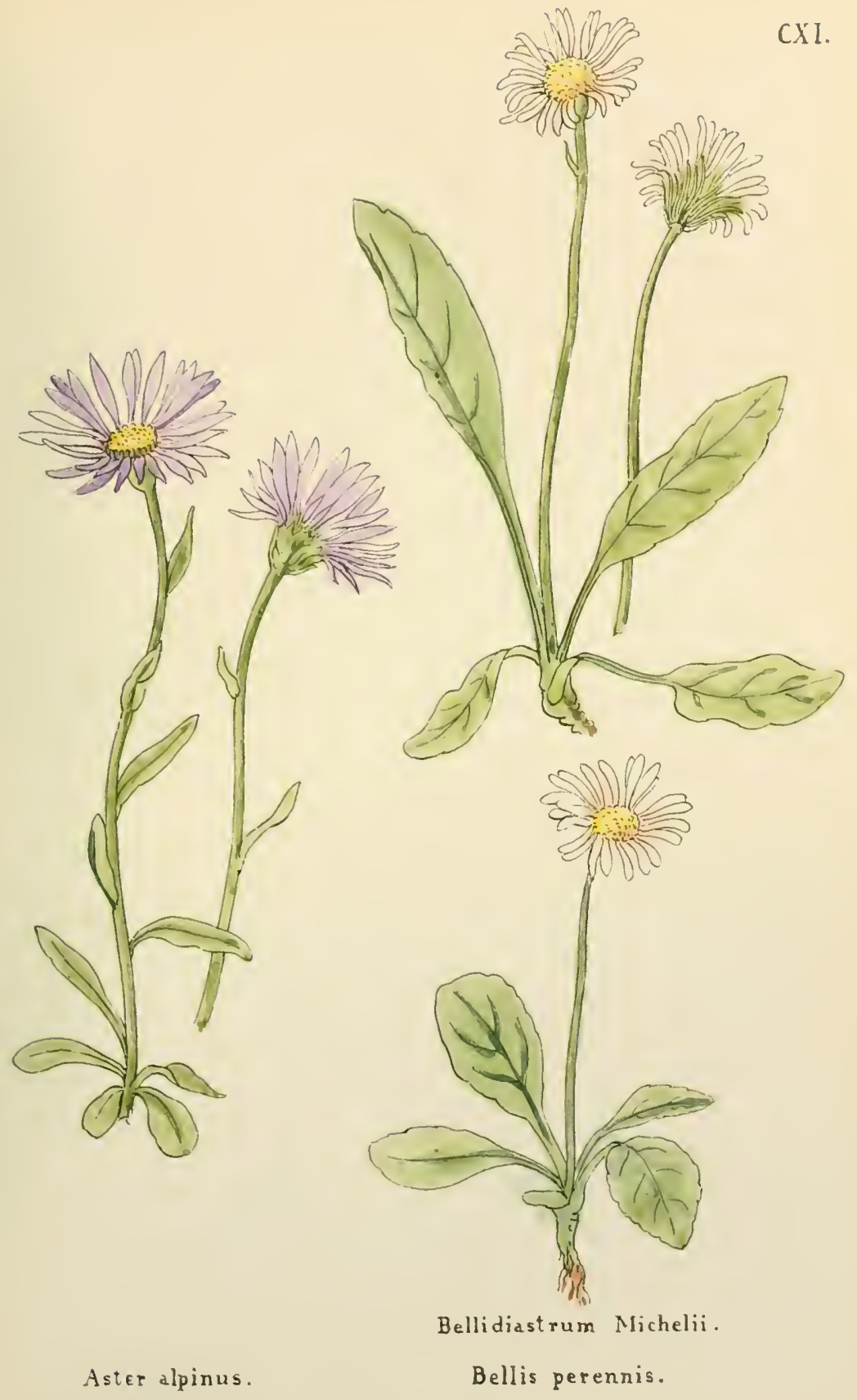





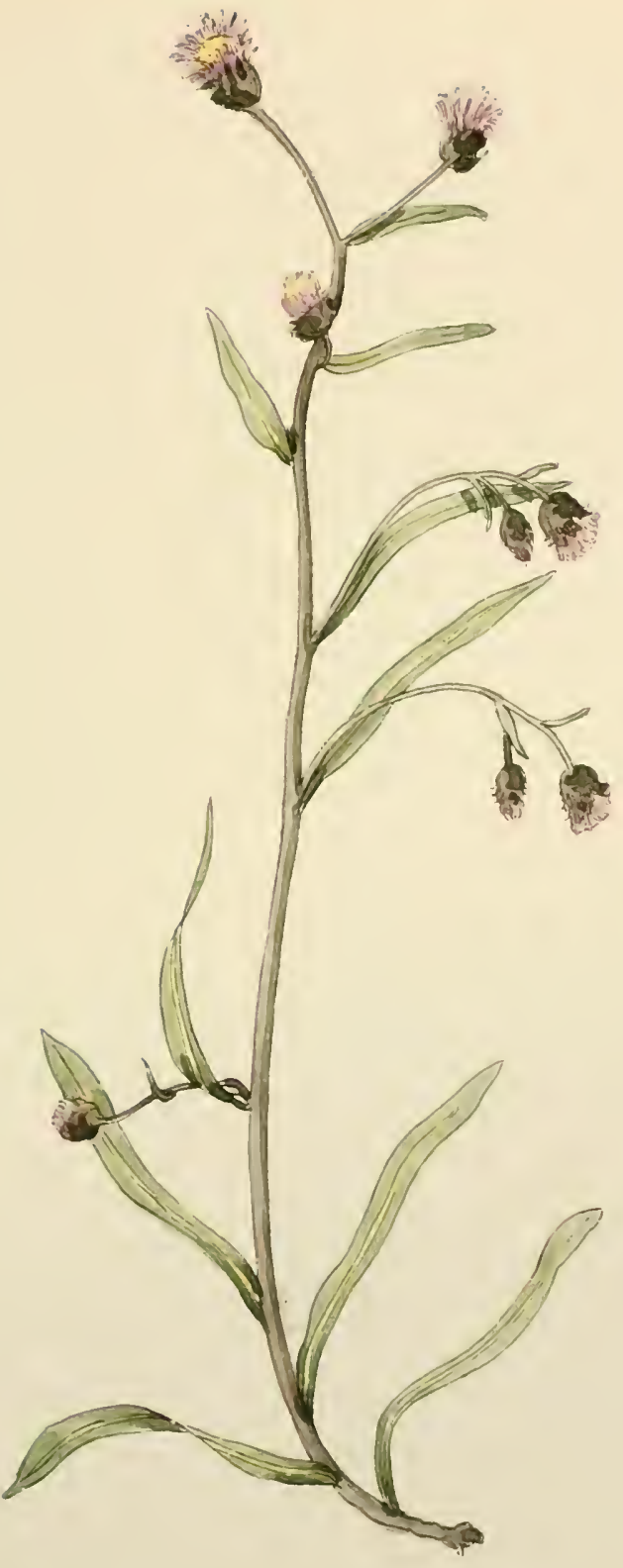

CXII. 

CXIII.

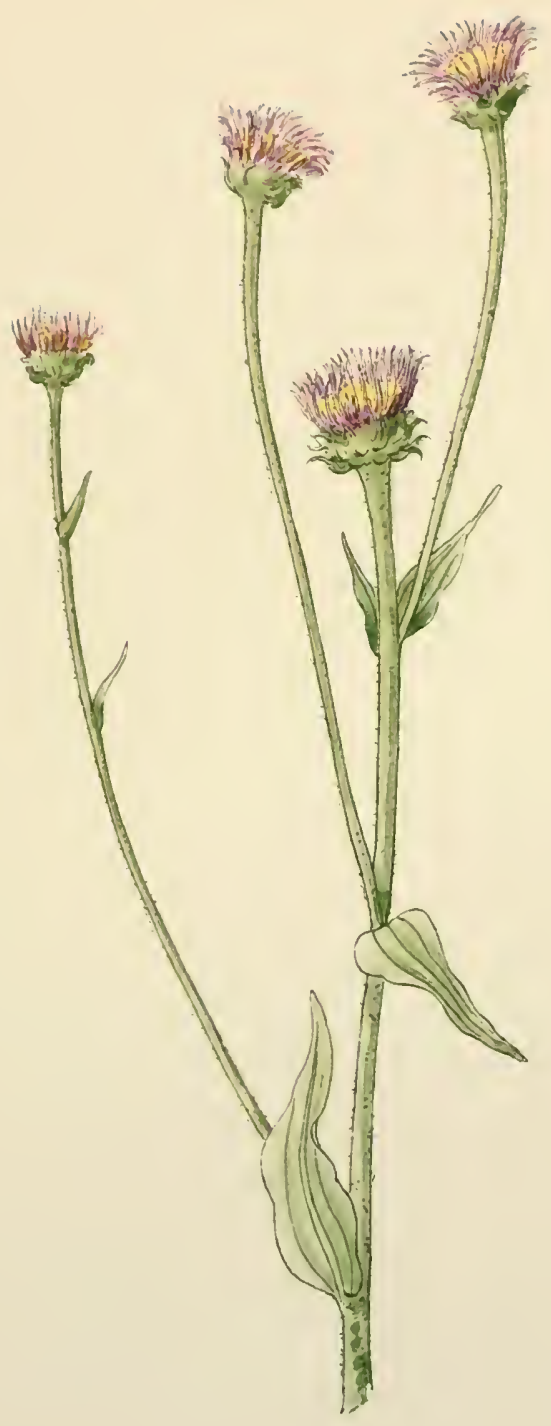

Erigeron Villarsii. 

cXIV.

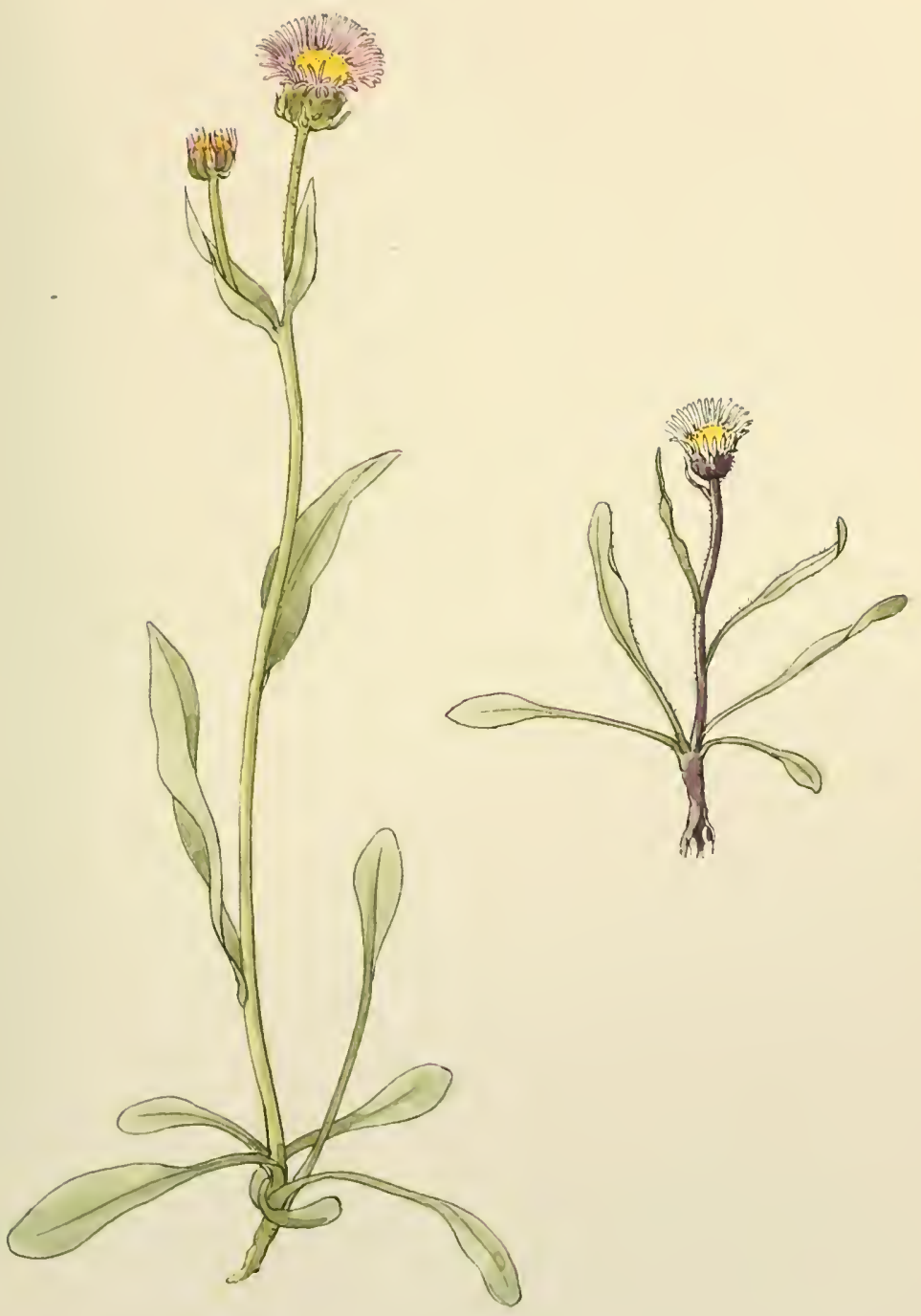

Erigeron alpinus. Erigeron uniflorus. 



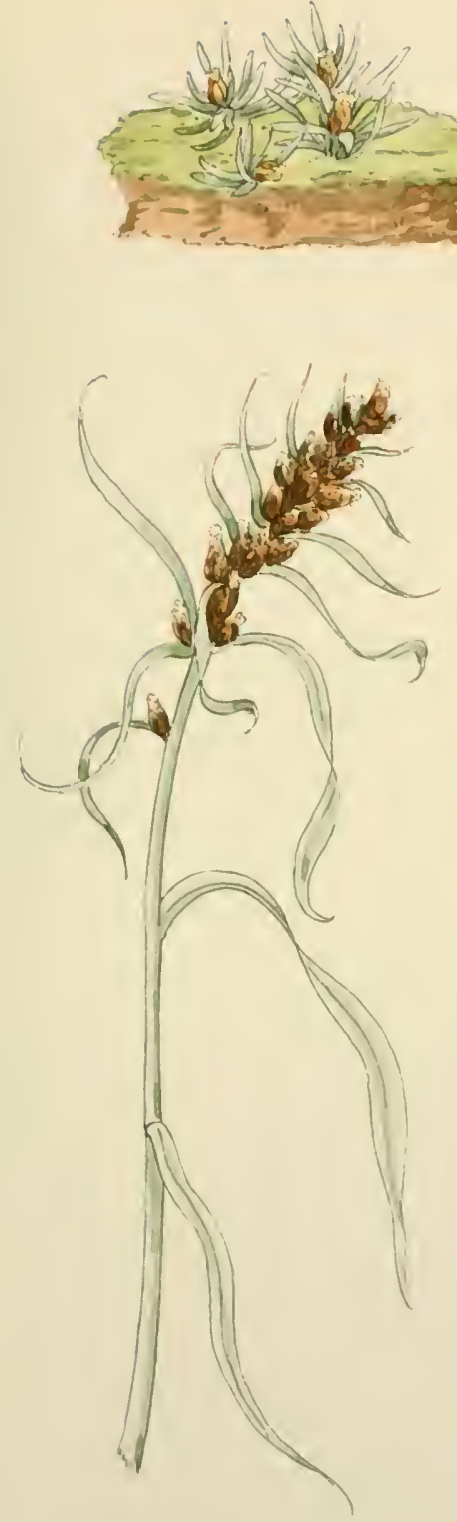

Gnaphalium supinum .

Gnaphalium sylvaticum .
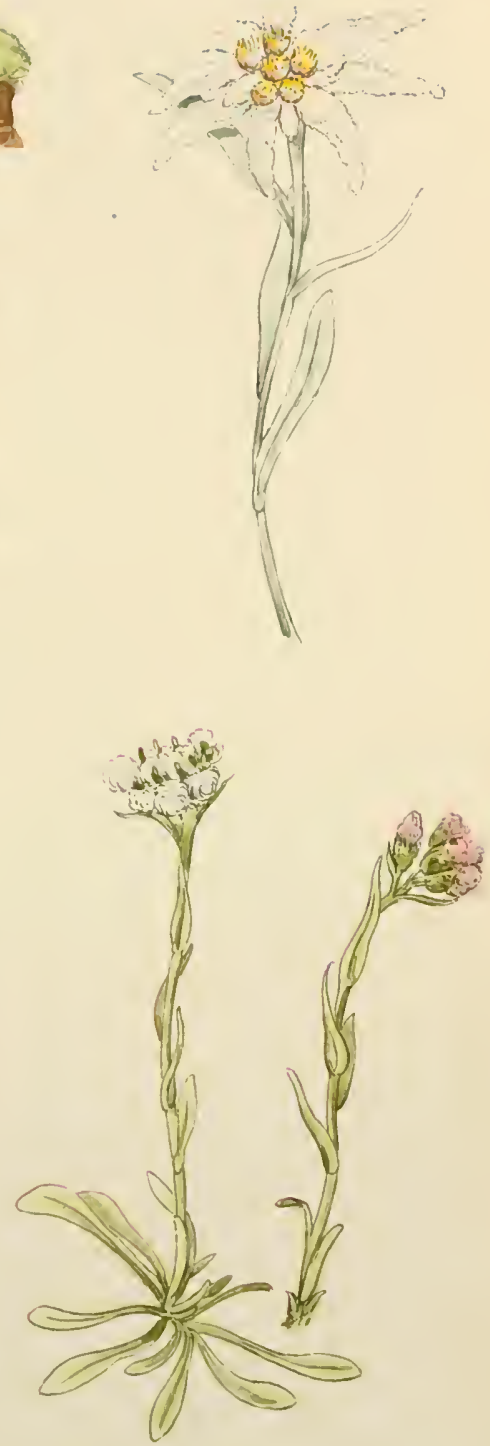

Gnsphalium Leontopodium. Gnapbalium dioicum . 


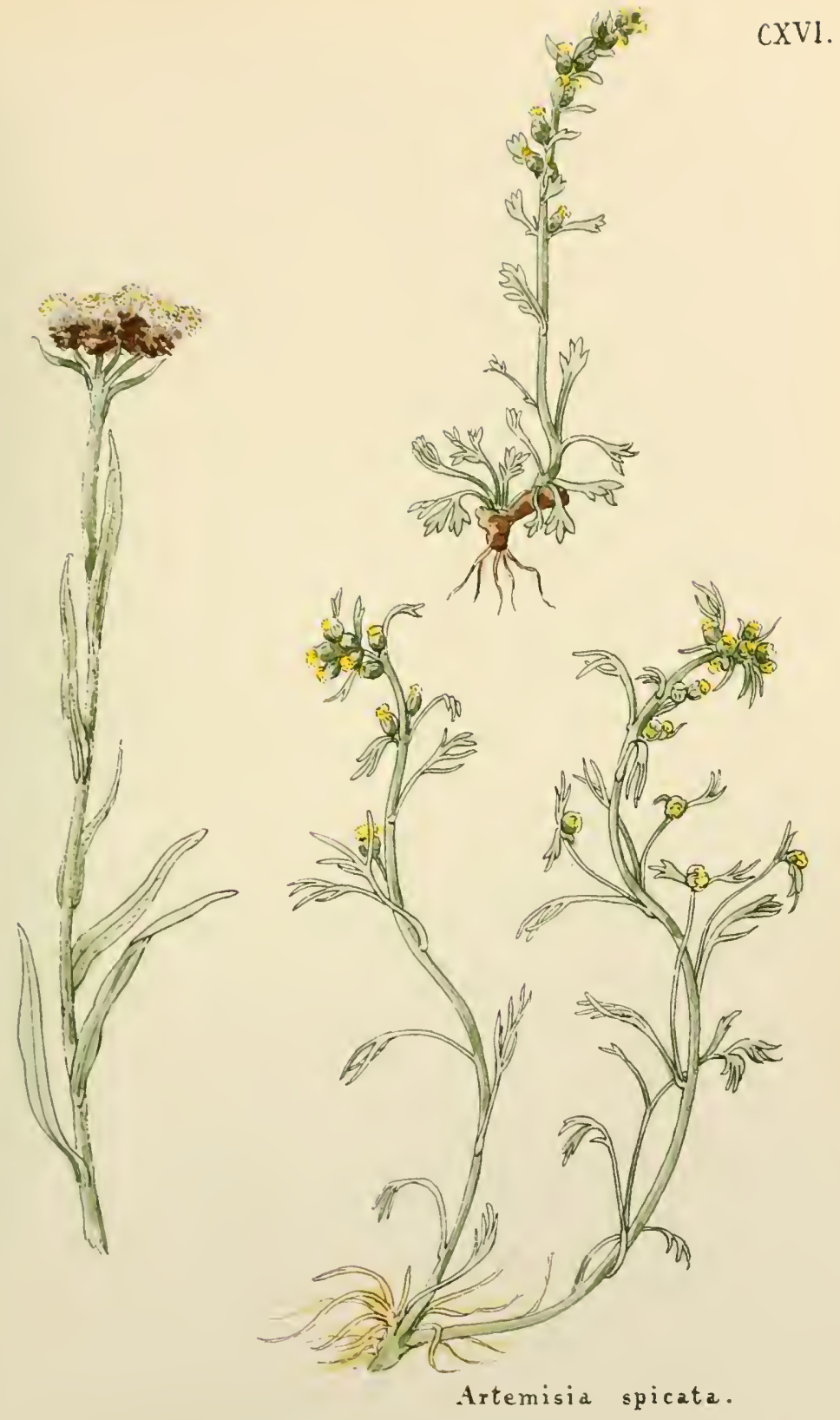

Graphalium carpathicum .

Artemisia mutellina. 


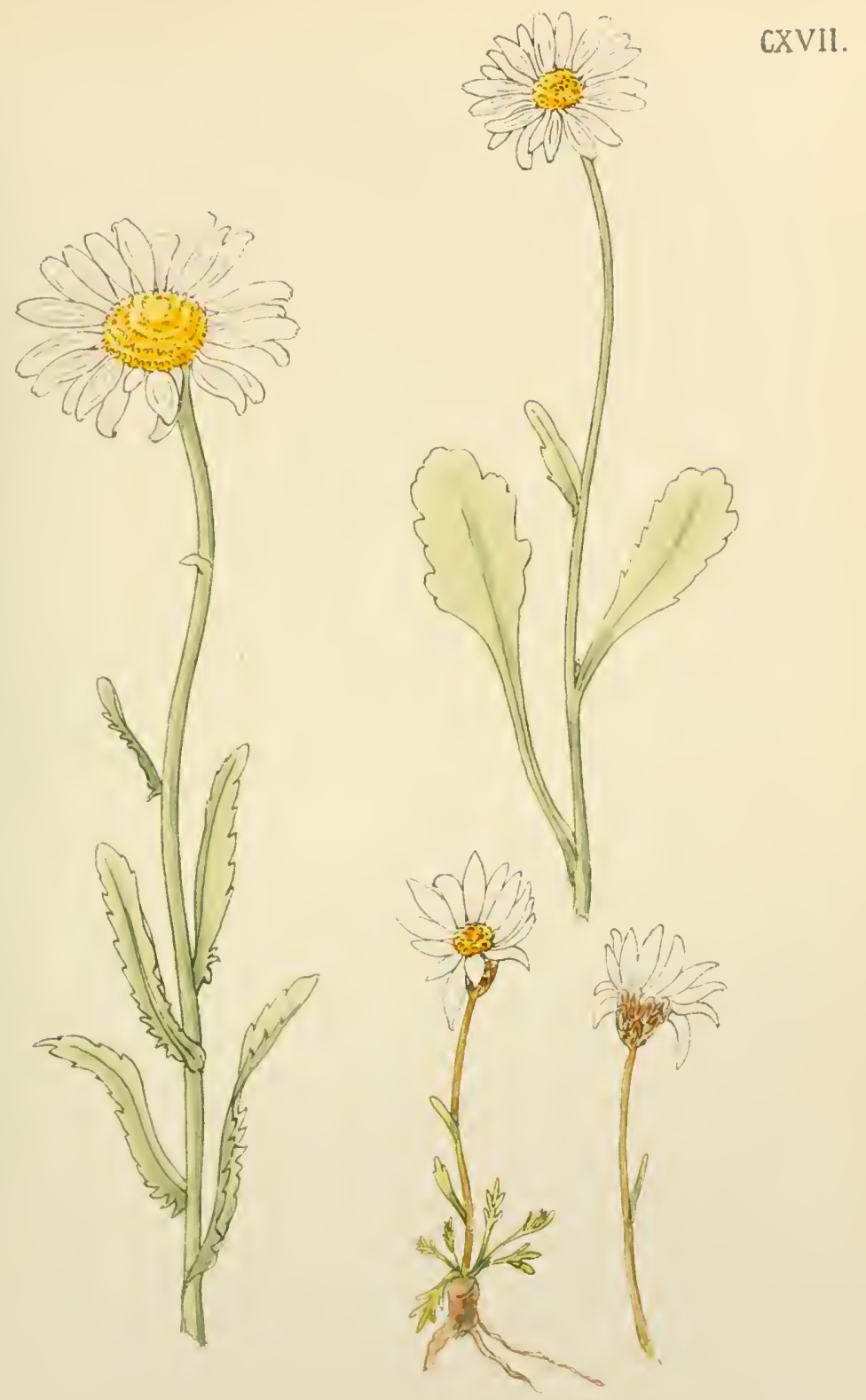

Leucanthemum coronopifolium.

Leucanthemum vulgare.

Leucanthenum alpinum . 



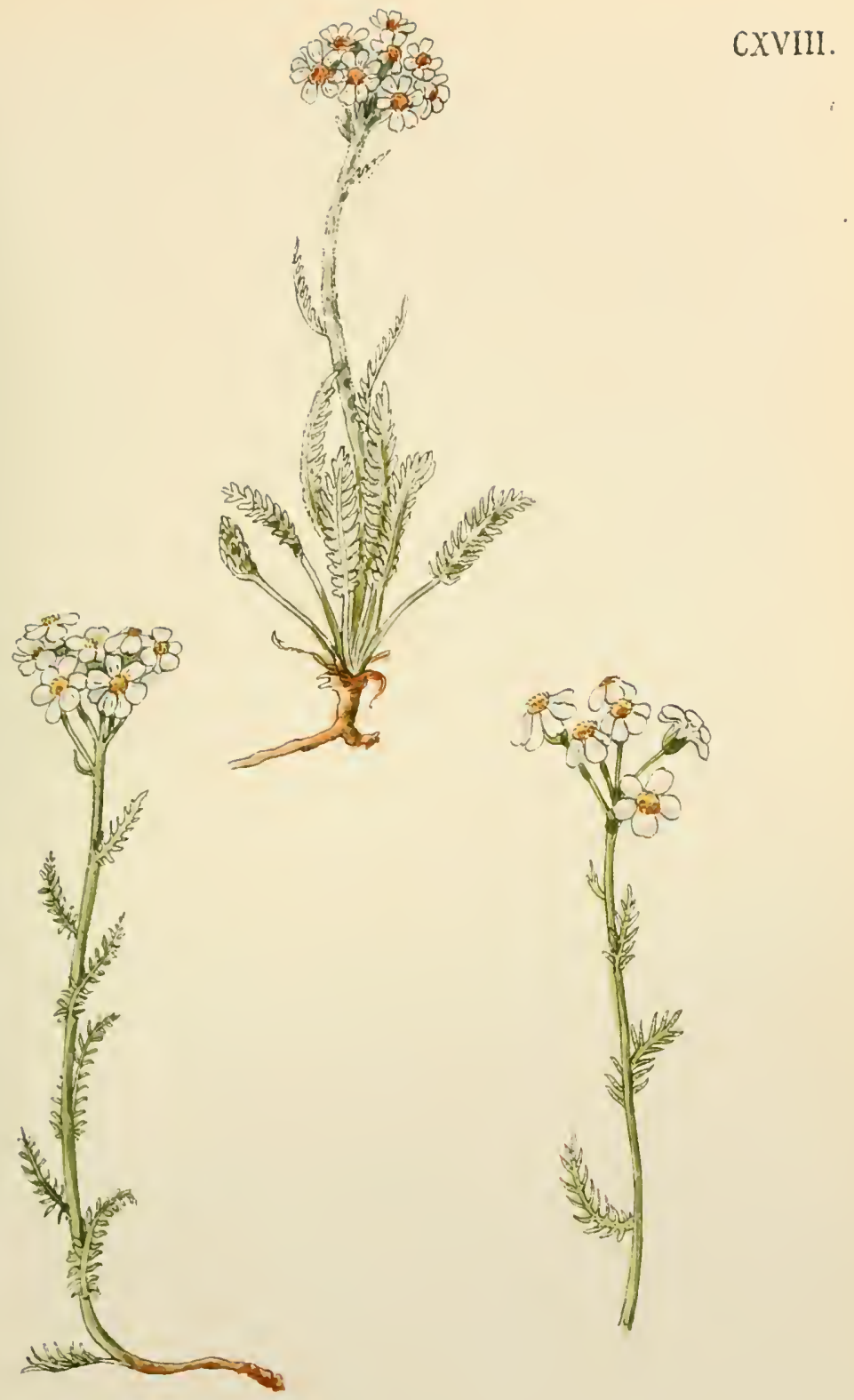

Achillea nana.

Achillea hybrida.

Achillea moschata. 



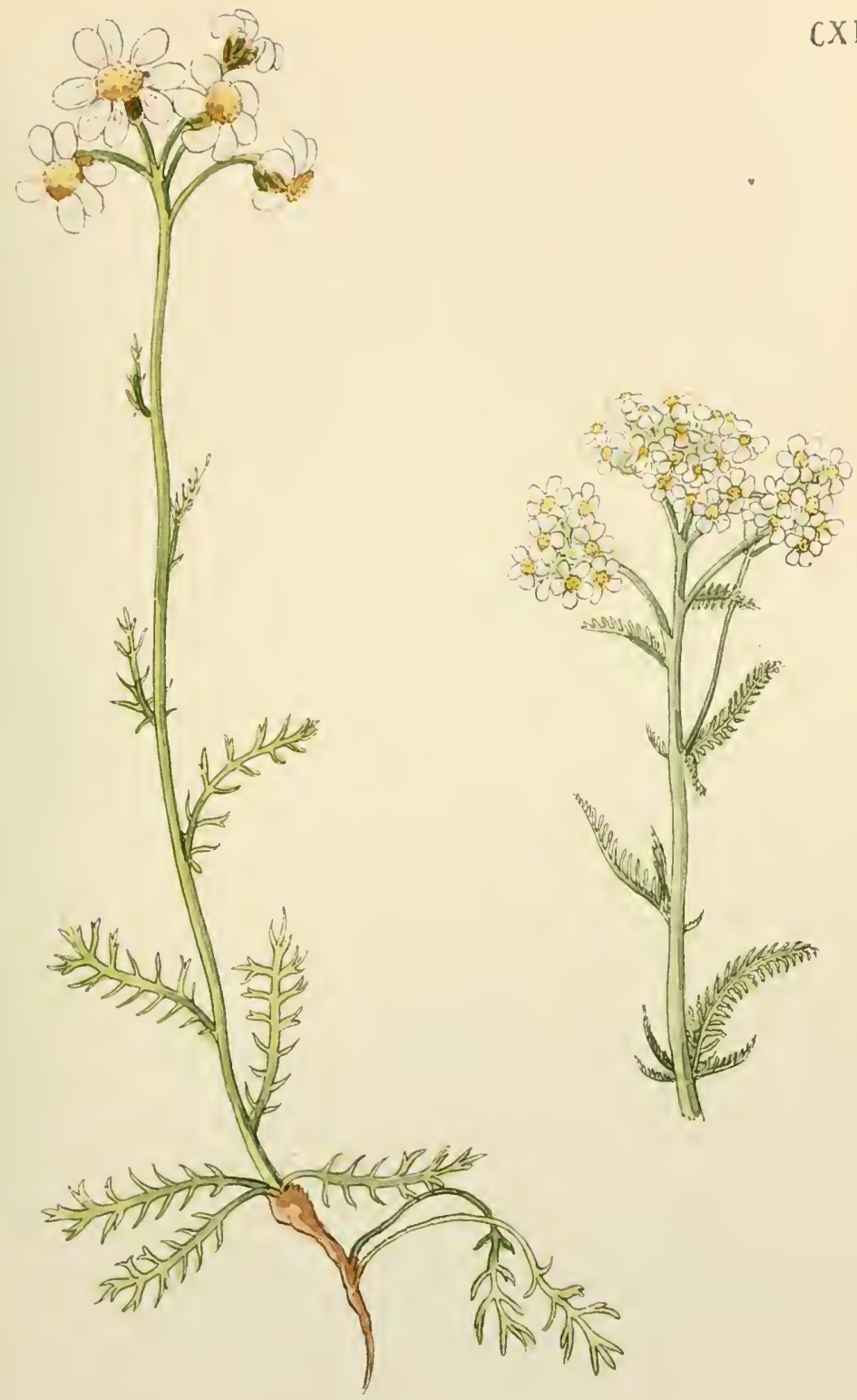

Achillea atrata.

Achillea Millefolium. 

CXX.

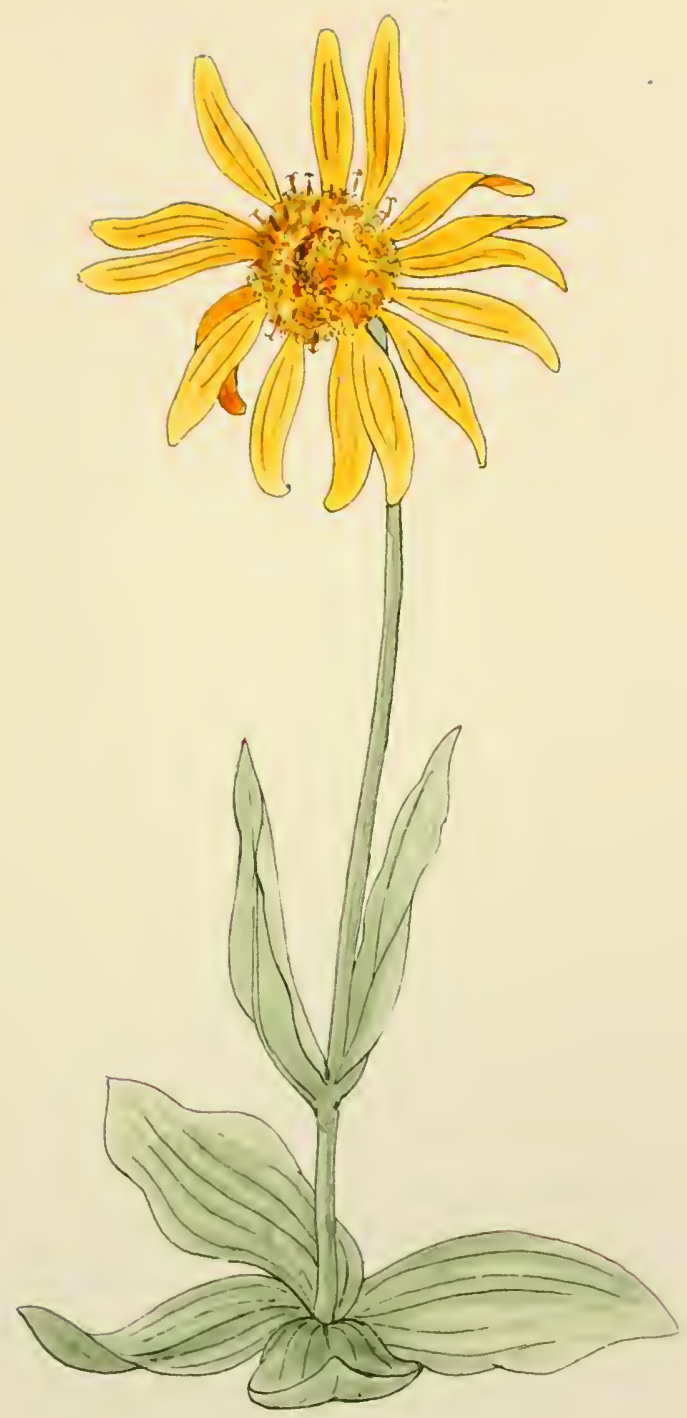

Arnica montana. 



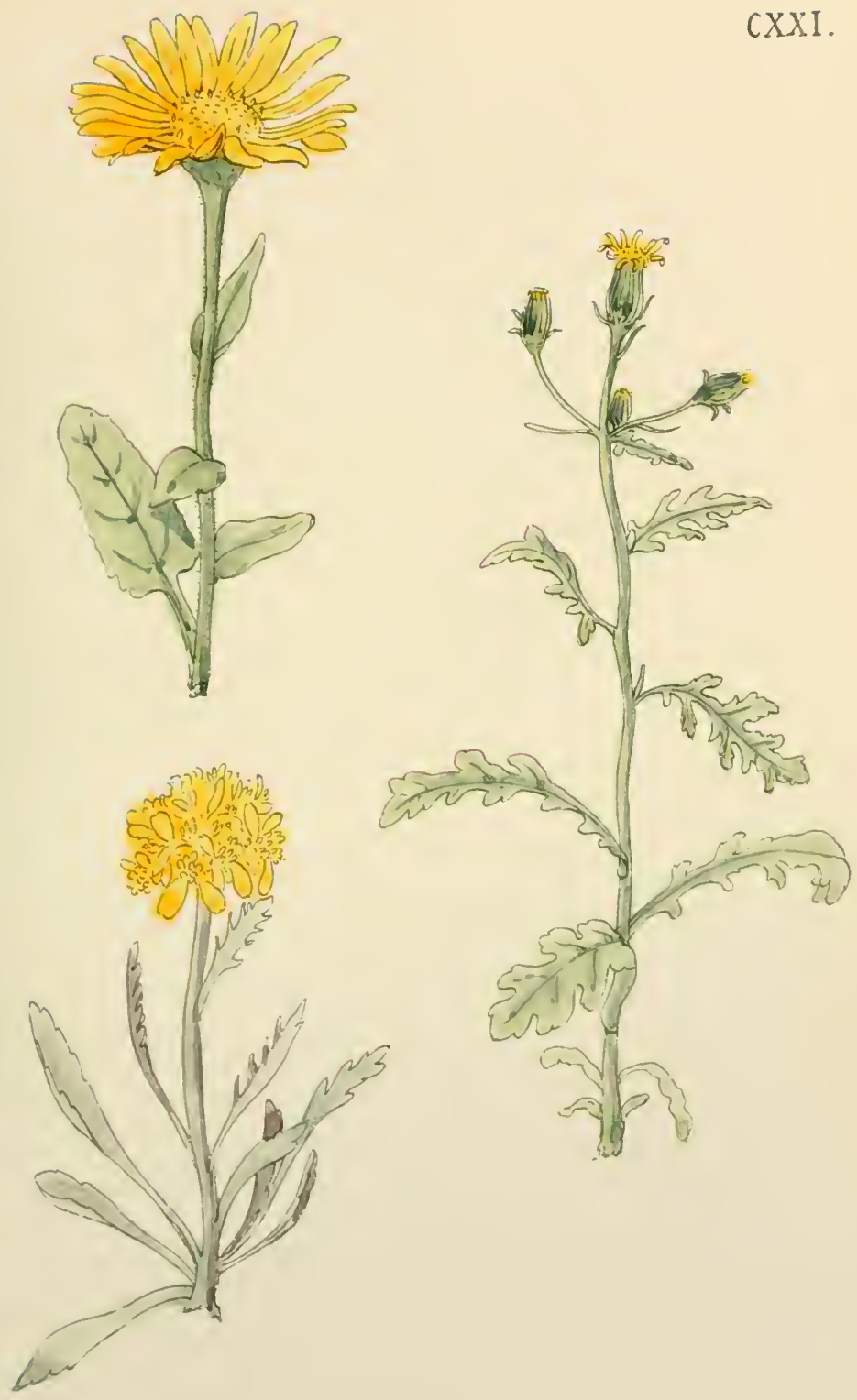

Aronicum Clusii.

Senecio incanus var. carniolicus. Senecio viscosus. 

CXXII .

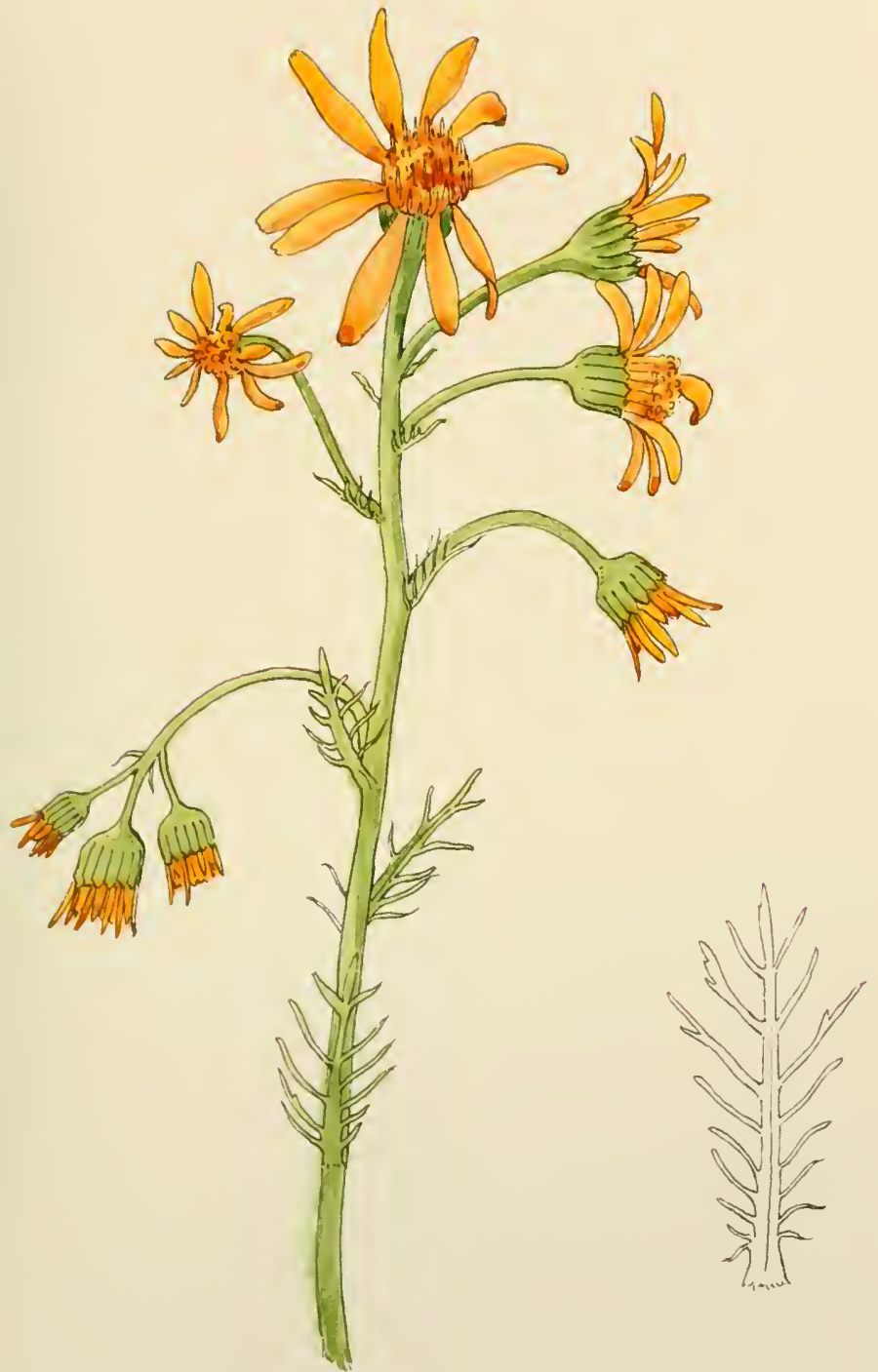

Senecio abrotanifolius. 



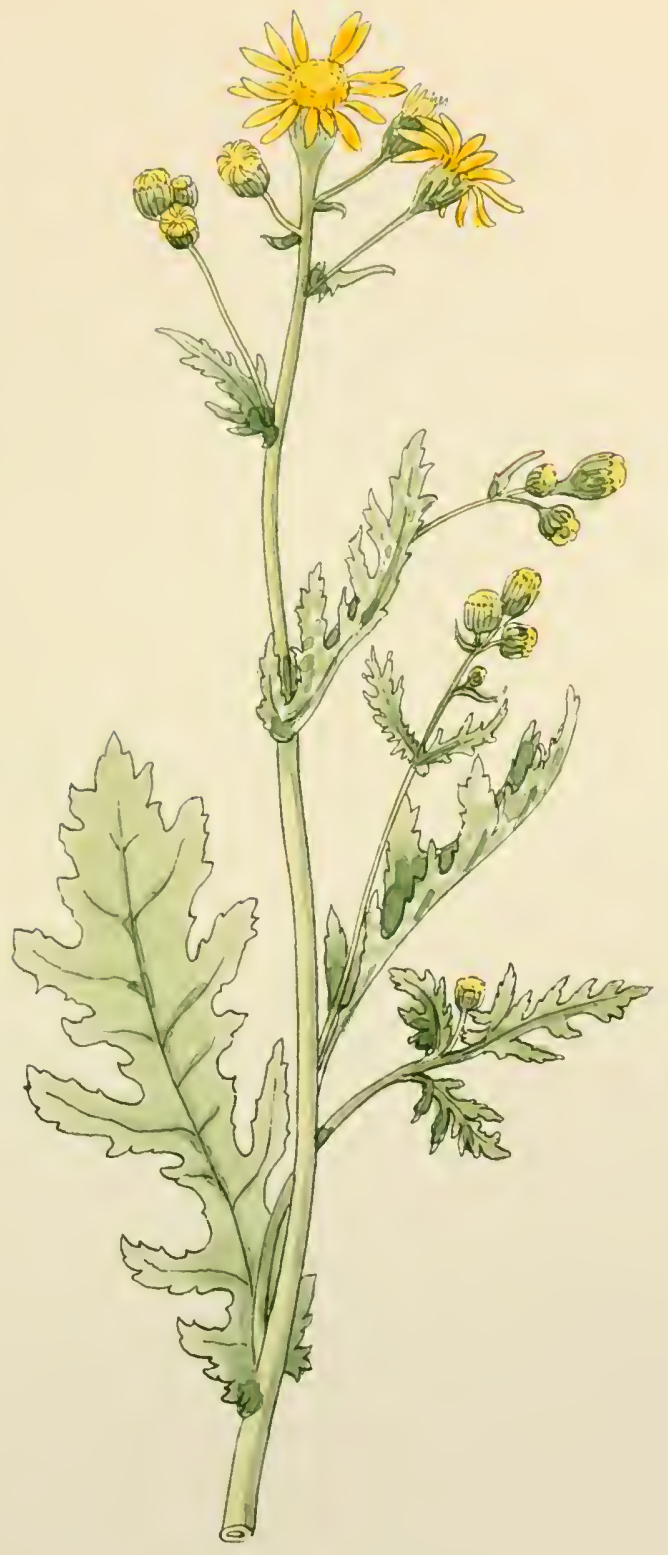

CXXIII. 
CXXIV.

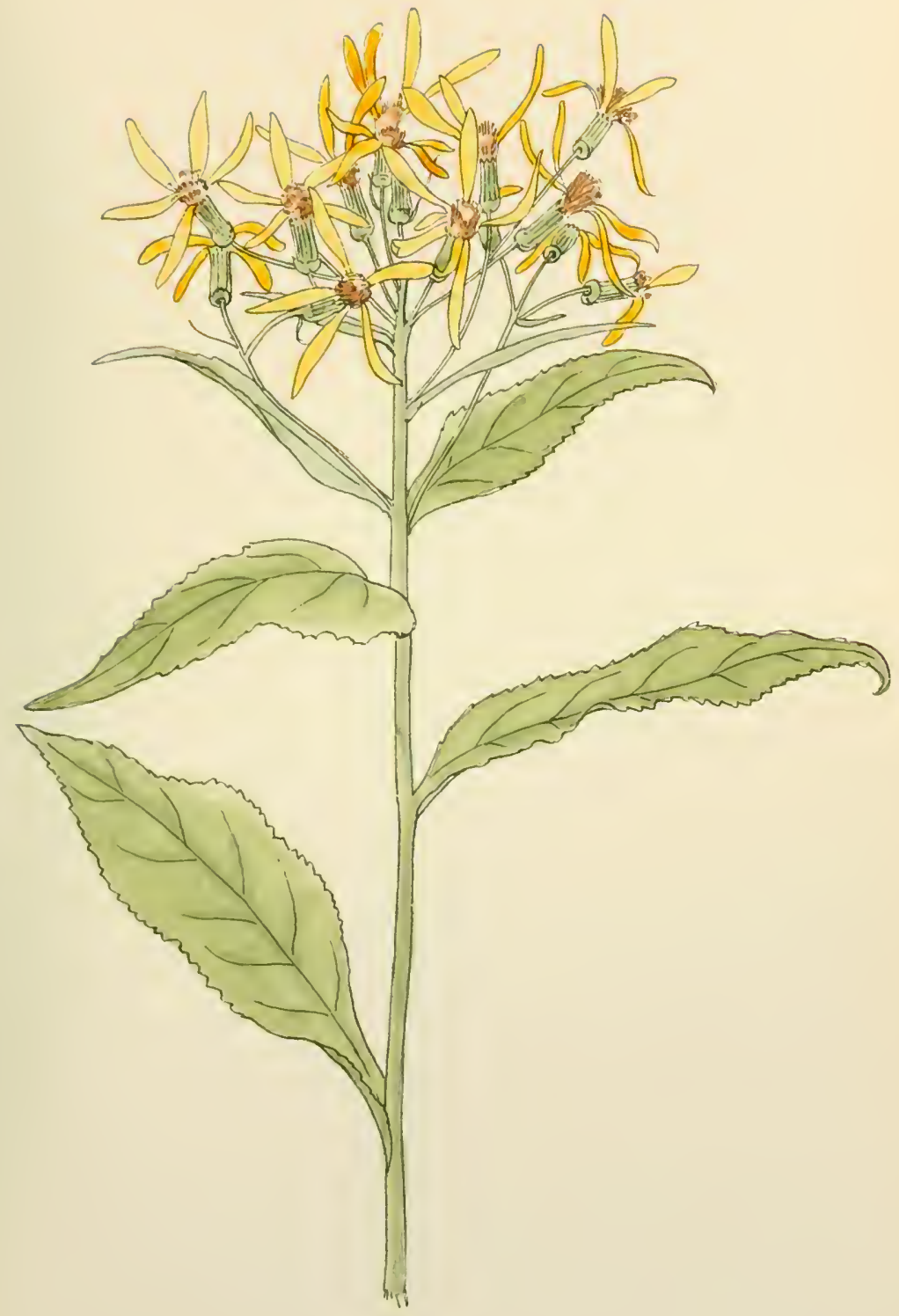

Senecio Fuchsii. 

CXXV.

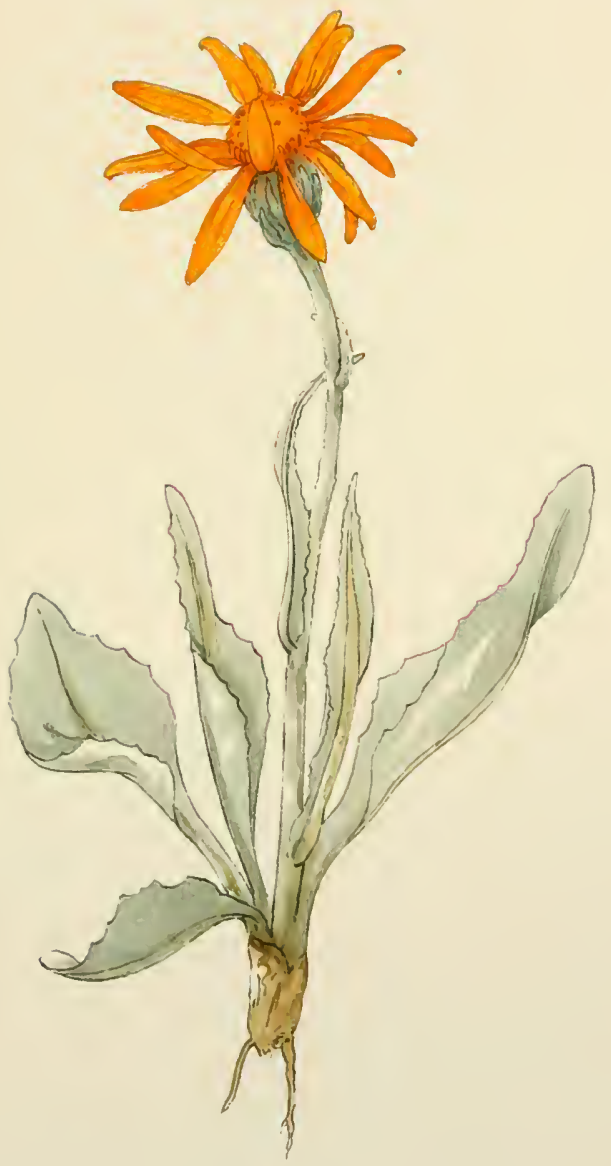

Senecio Doronicum. 



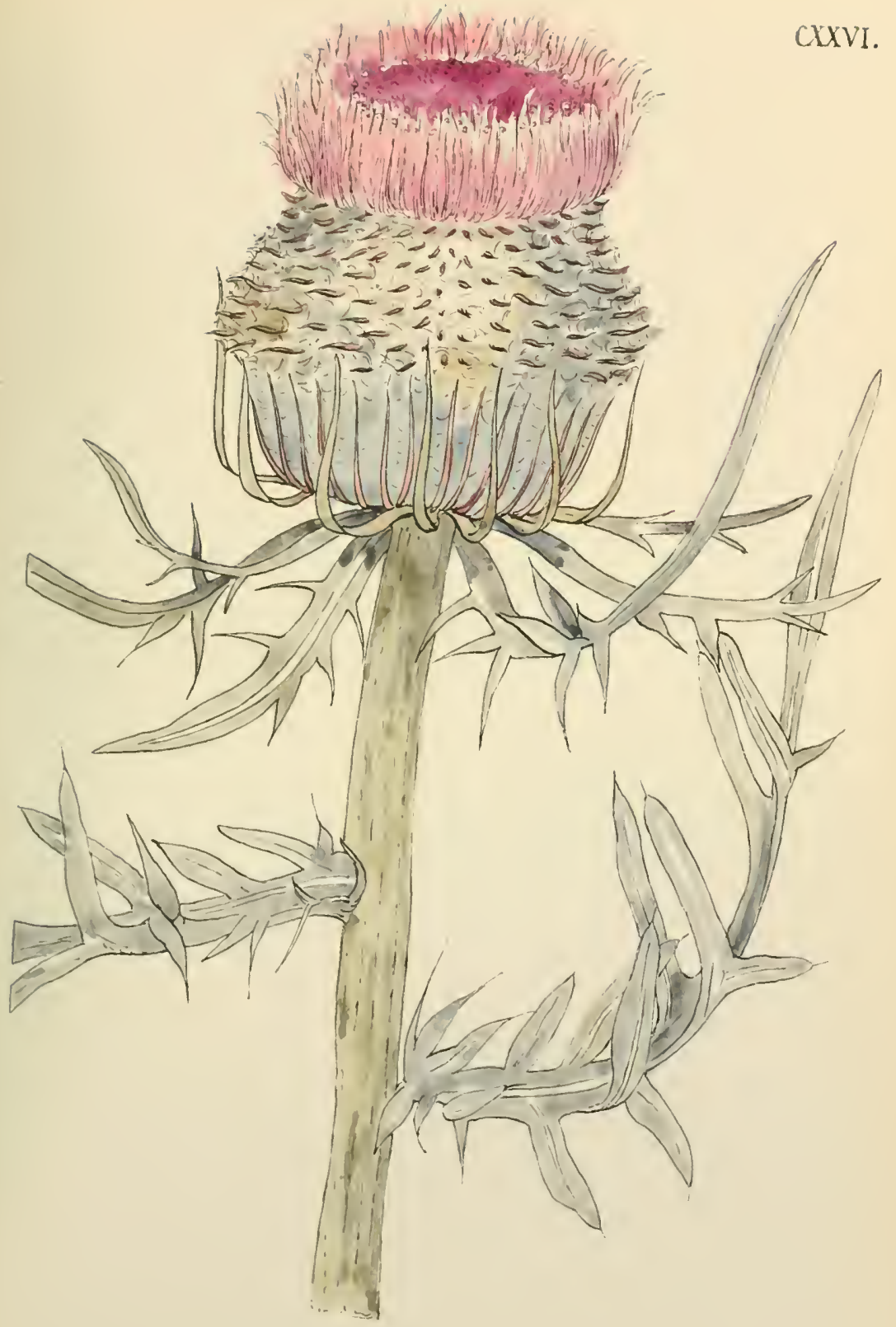

Cirsium eriophorusa. 
CXXVII.

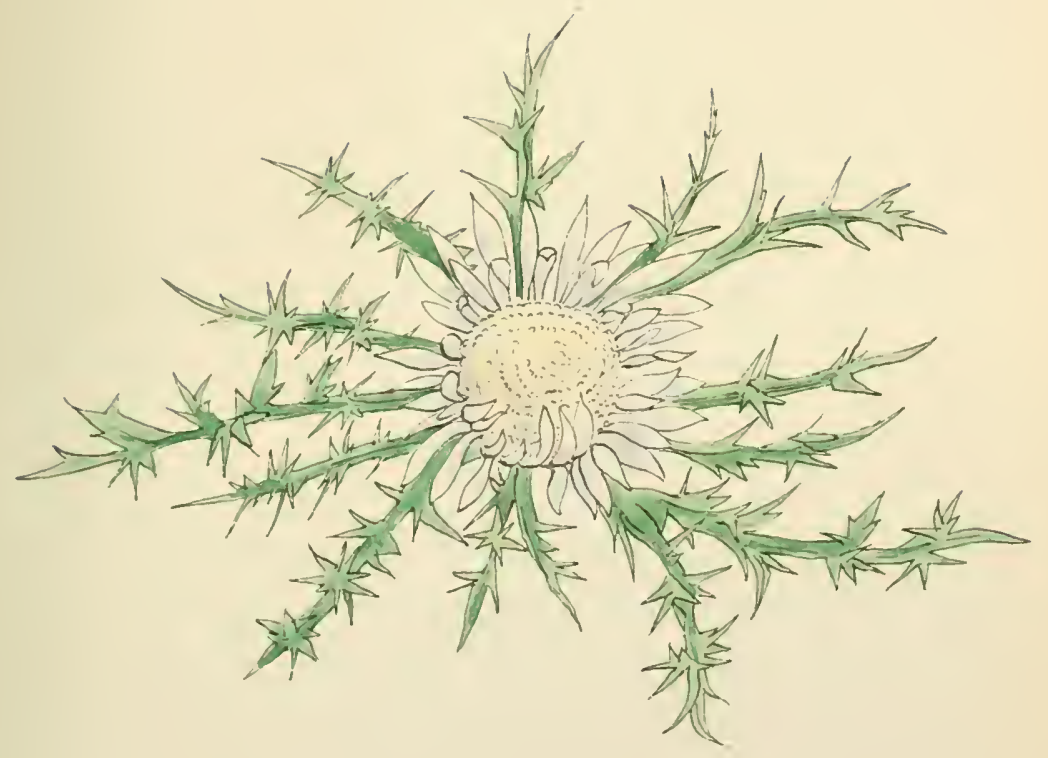

Carlina acaulis. 

CXXVIII

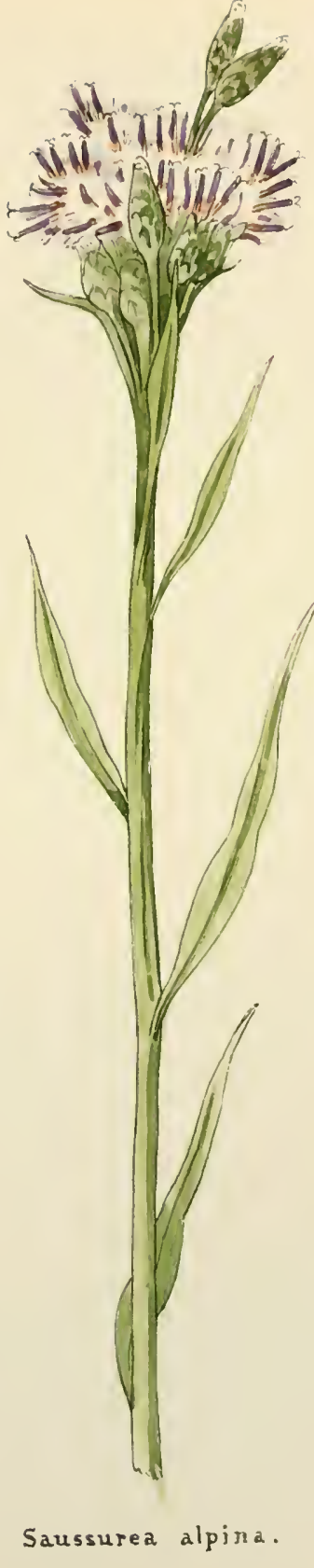




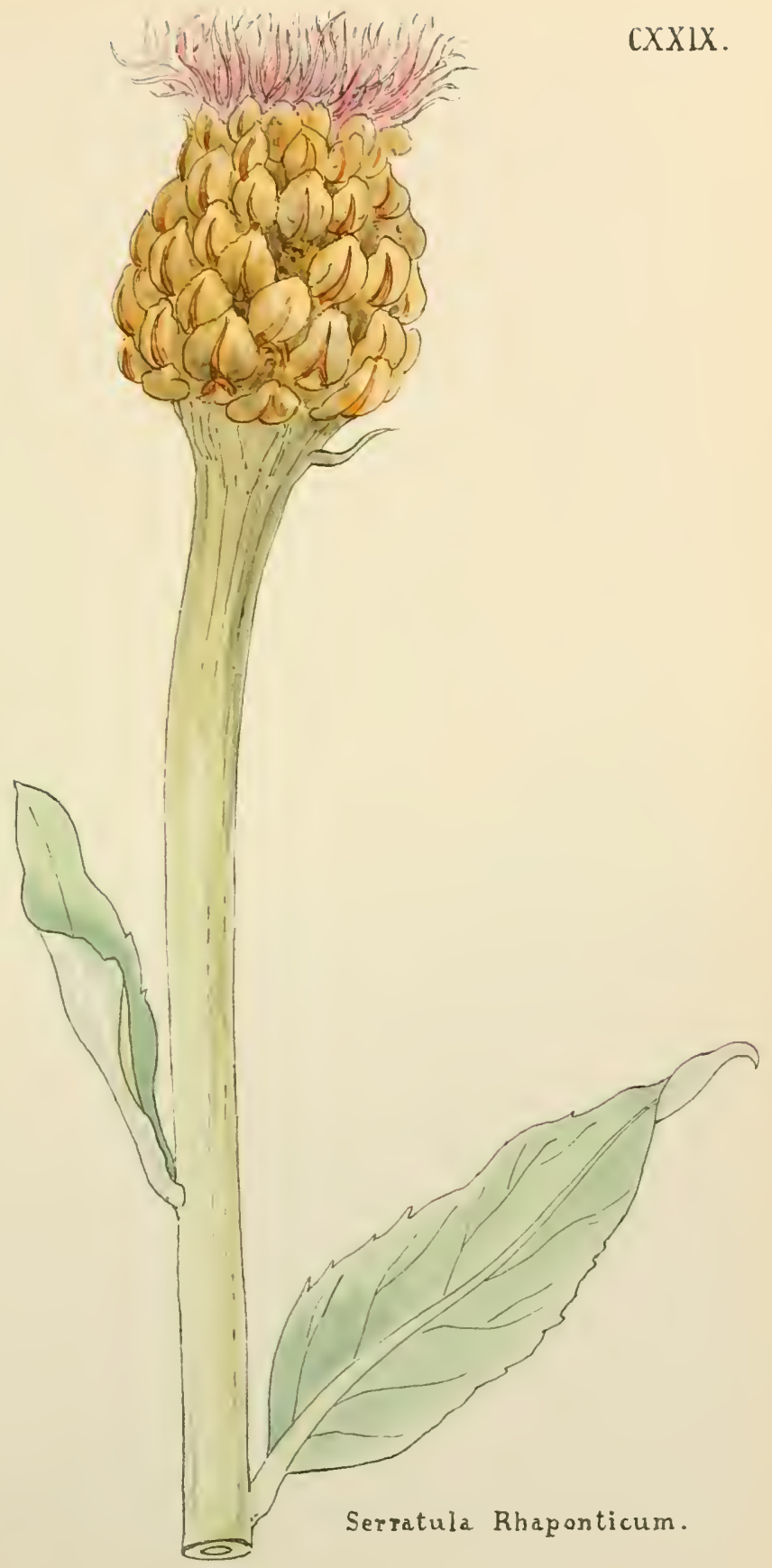





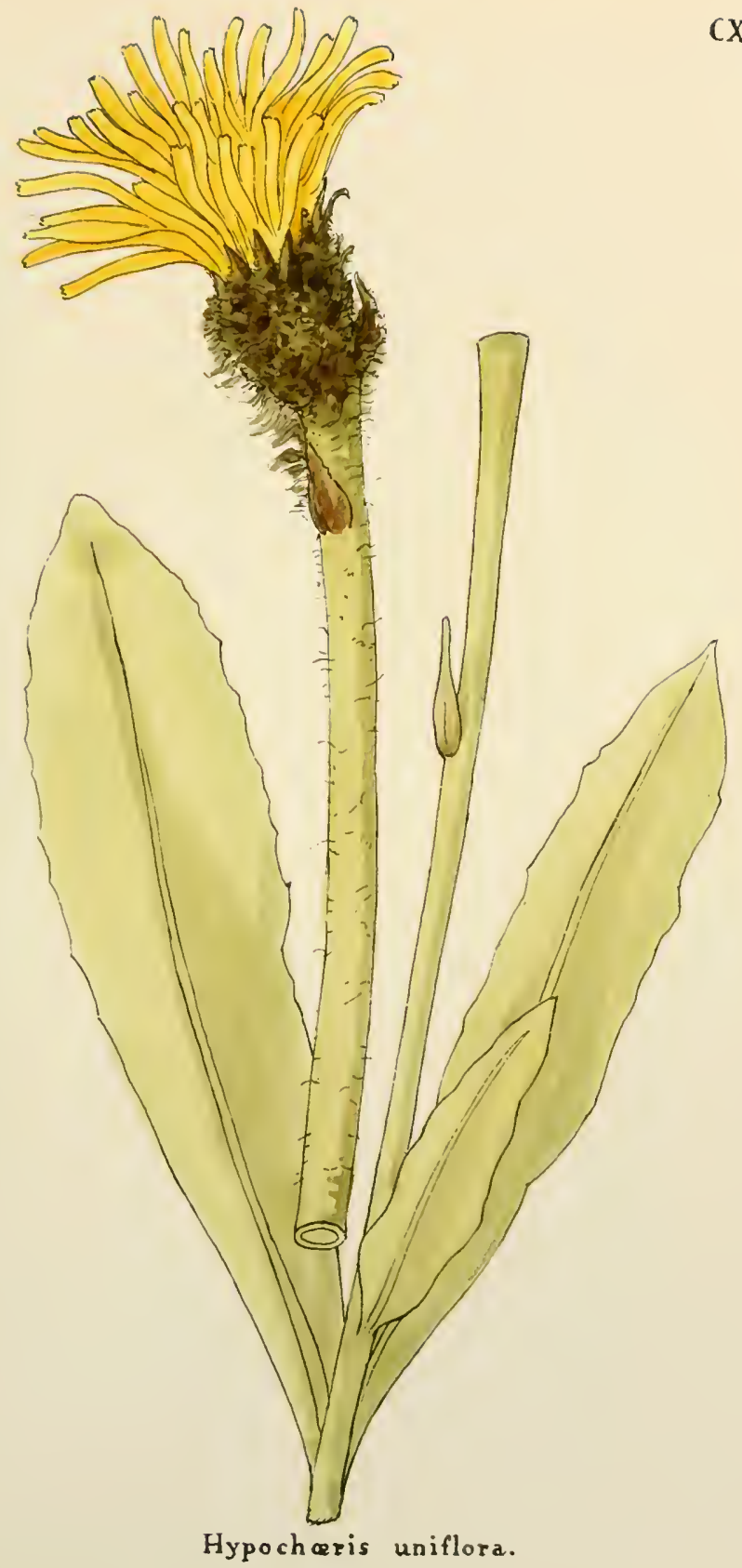

CXXX. 

CXXXI.

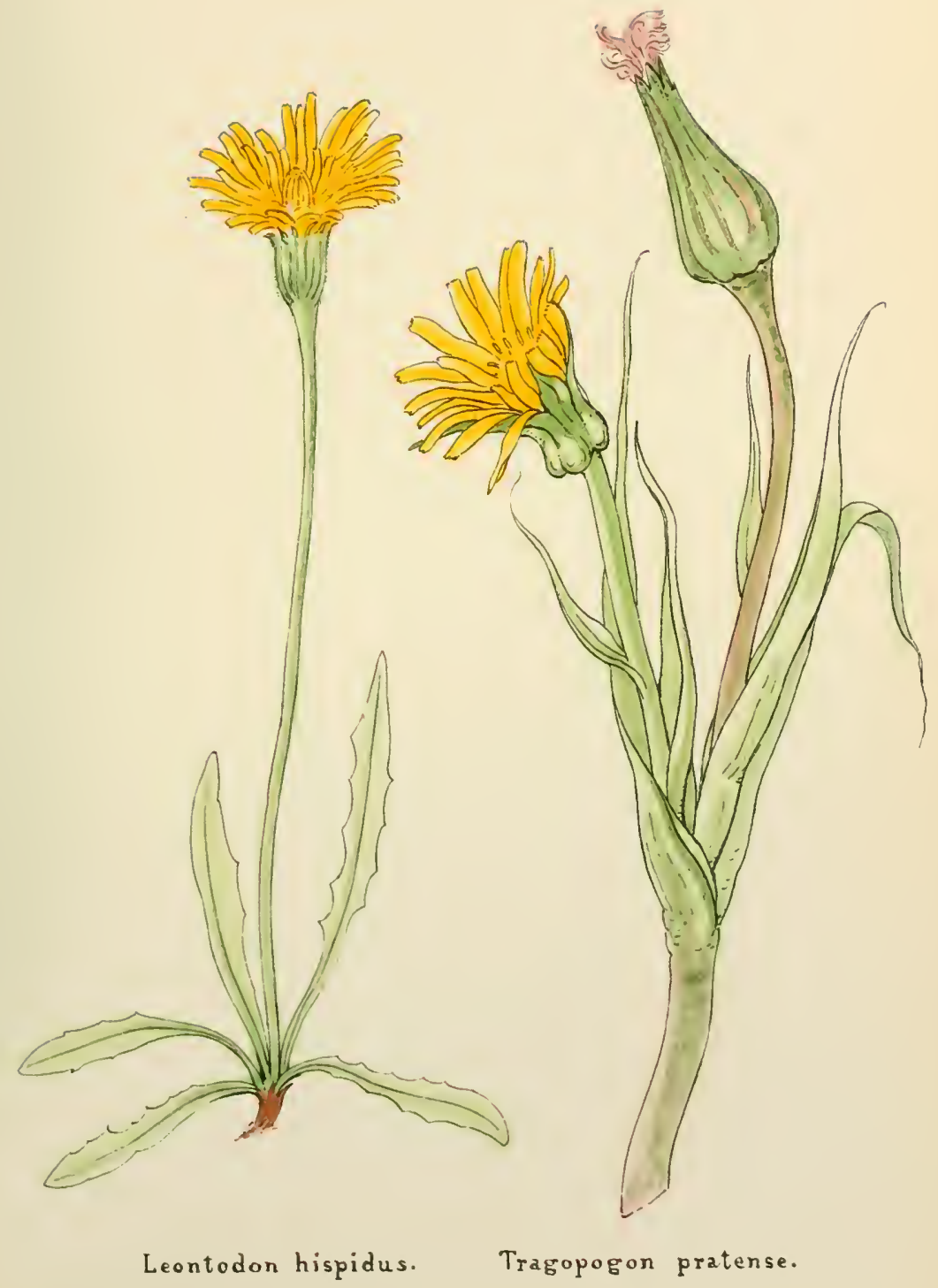



CXXXII.

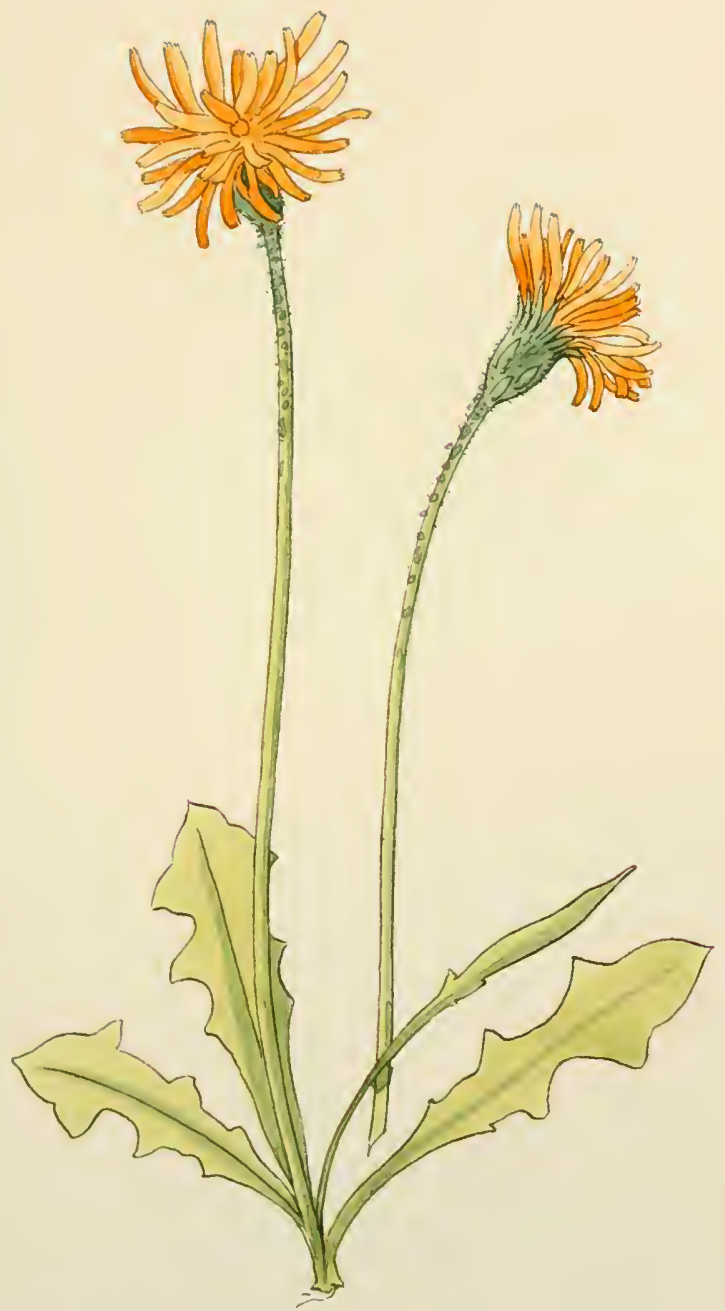

Crepis aurea. 
CXXXIII.
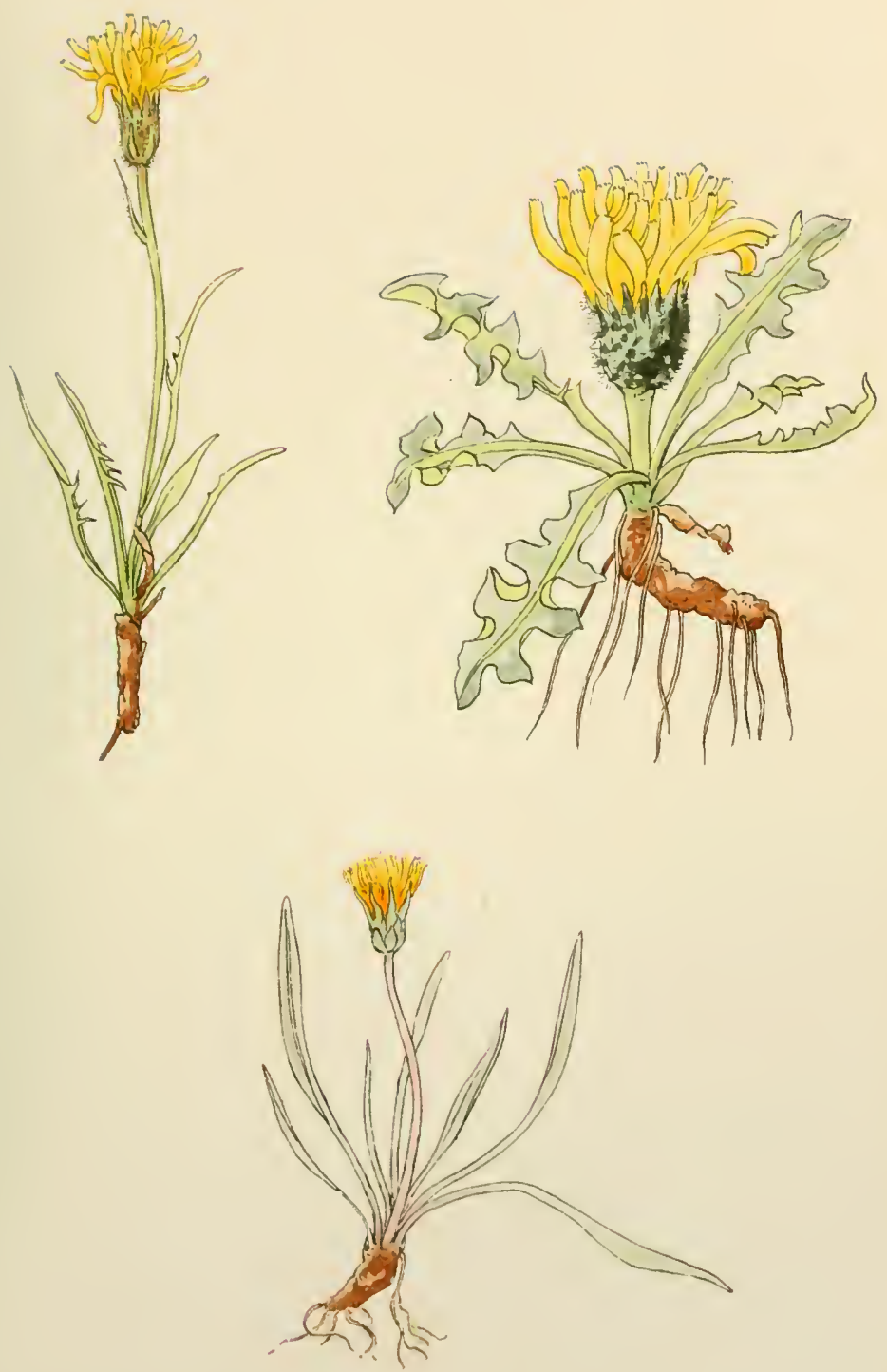

Crepis Jacquini.

Crepis hyoseridifolia.

Taraxacum officinale ver. palustre. 


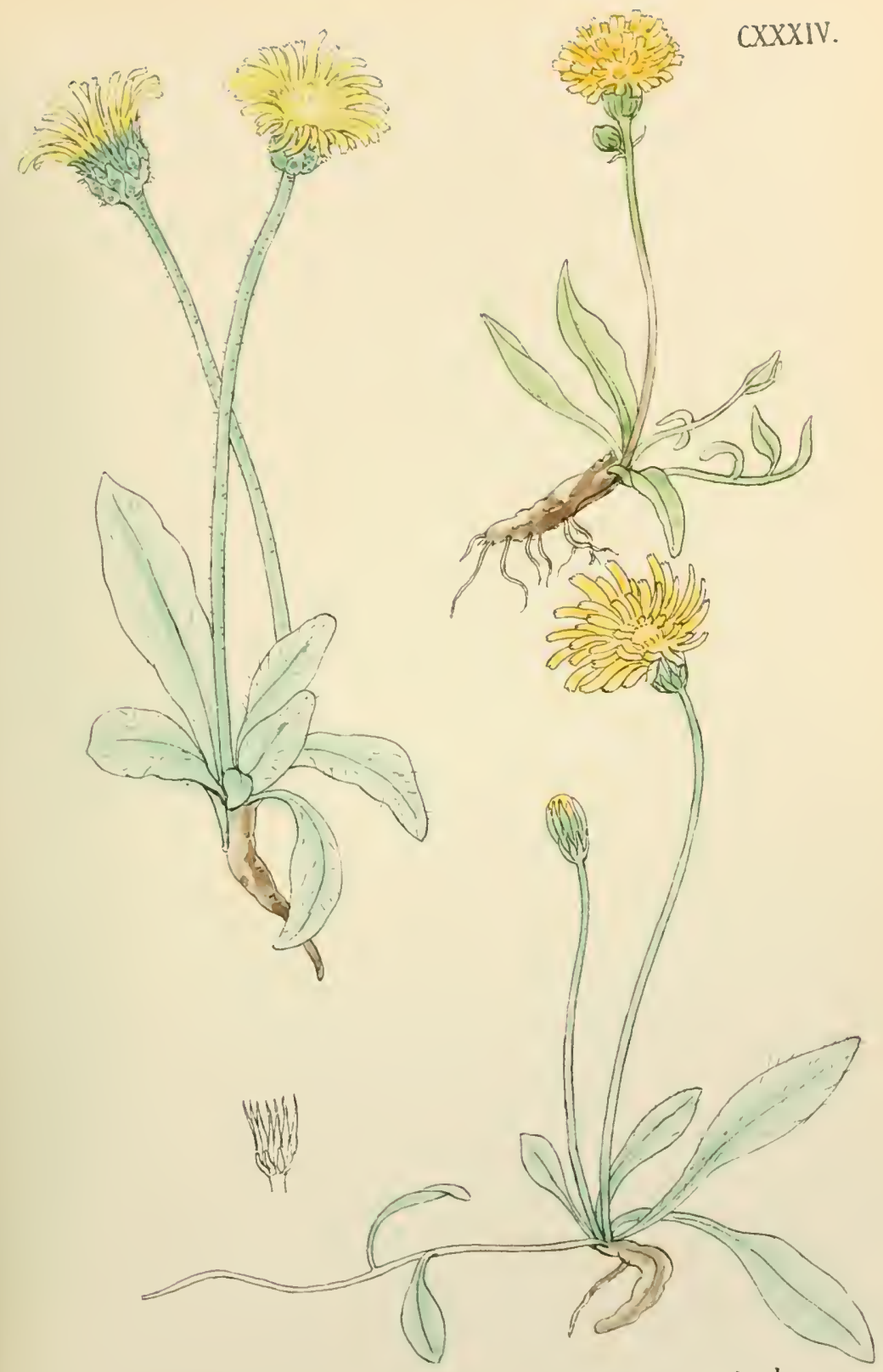

Hieracium piloselliforme. Hieracium Auricula.

Hieracium Pilosella. 


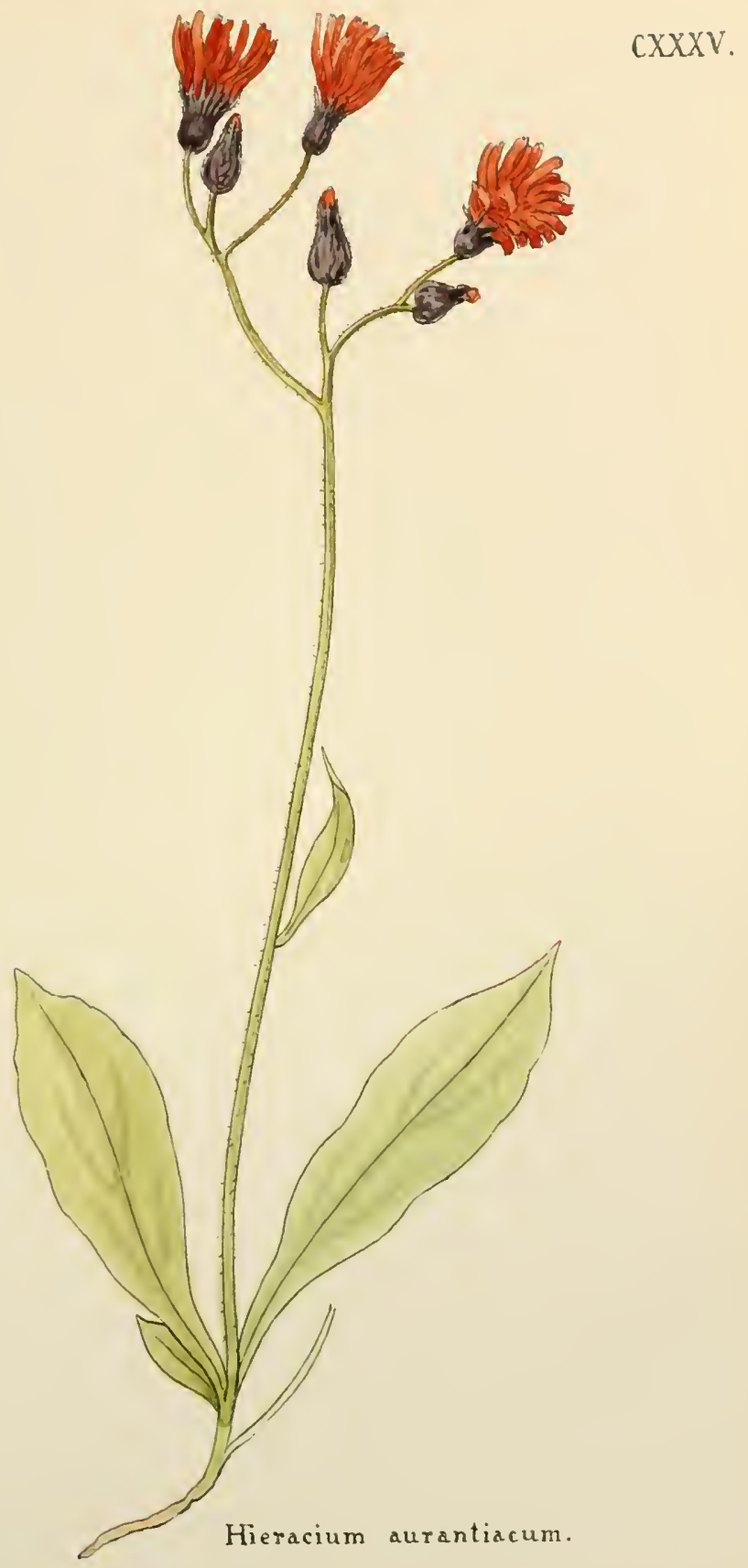



CXXXVI.
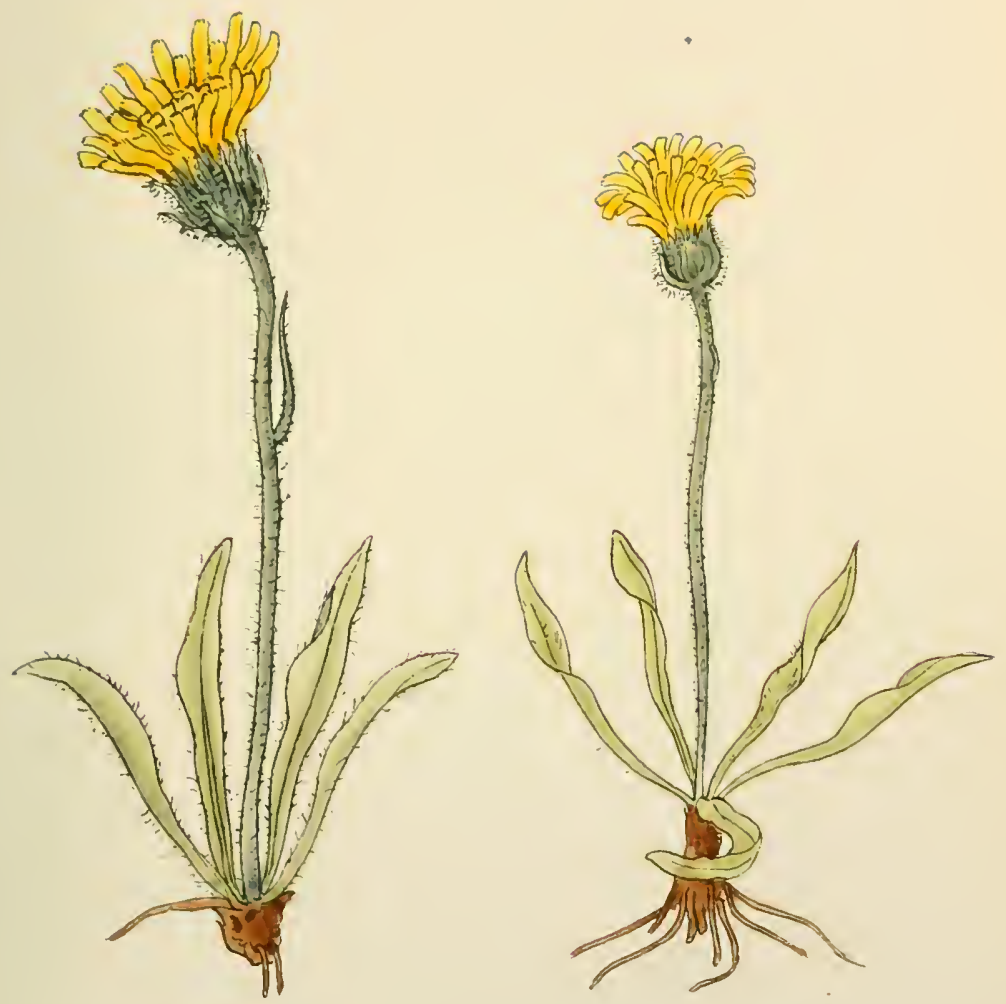

Hieracium alpinum.

Hieracium glanduliferum. 



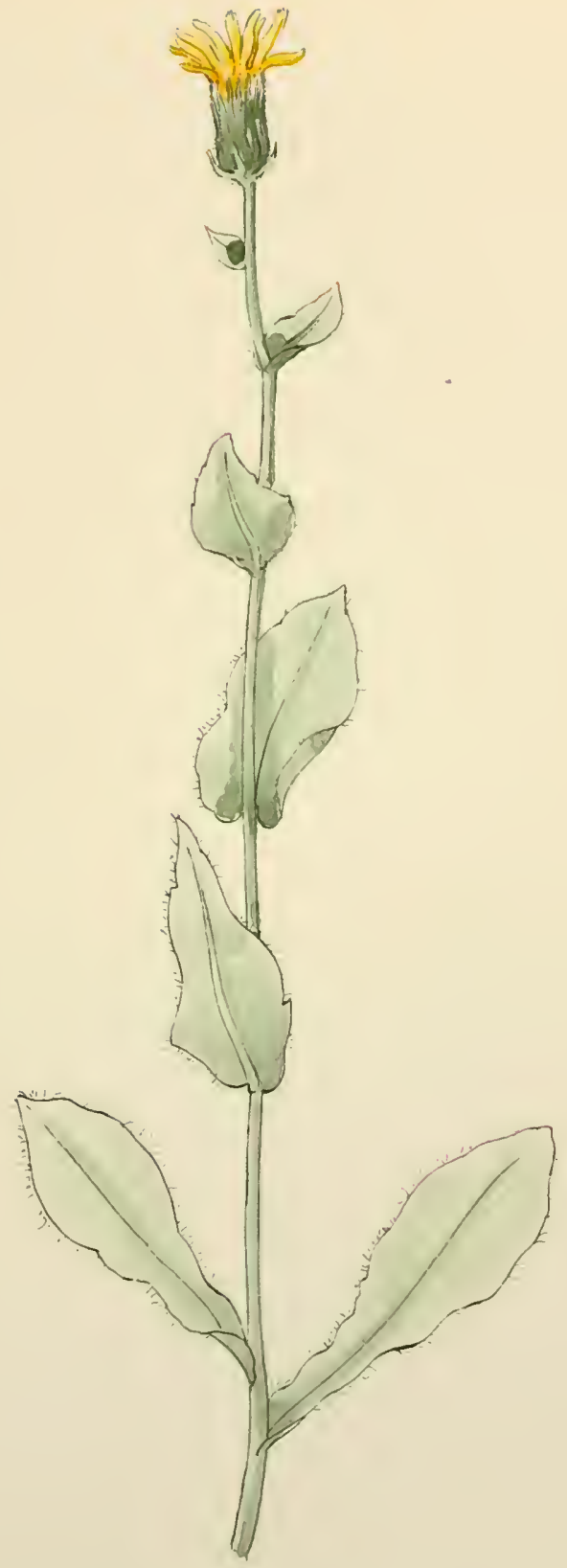

CXXXYII.

Hieracium villosum var dentatum. 
CXXXVIII.

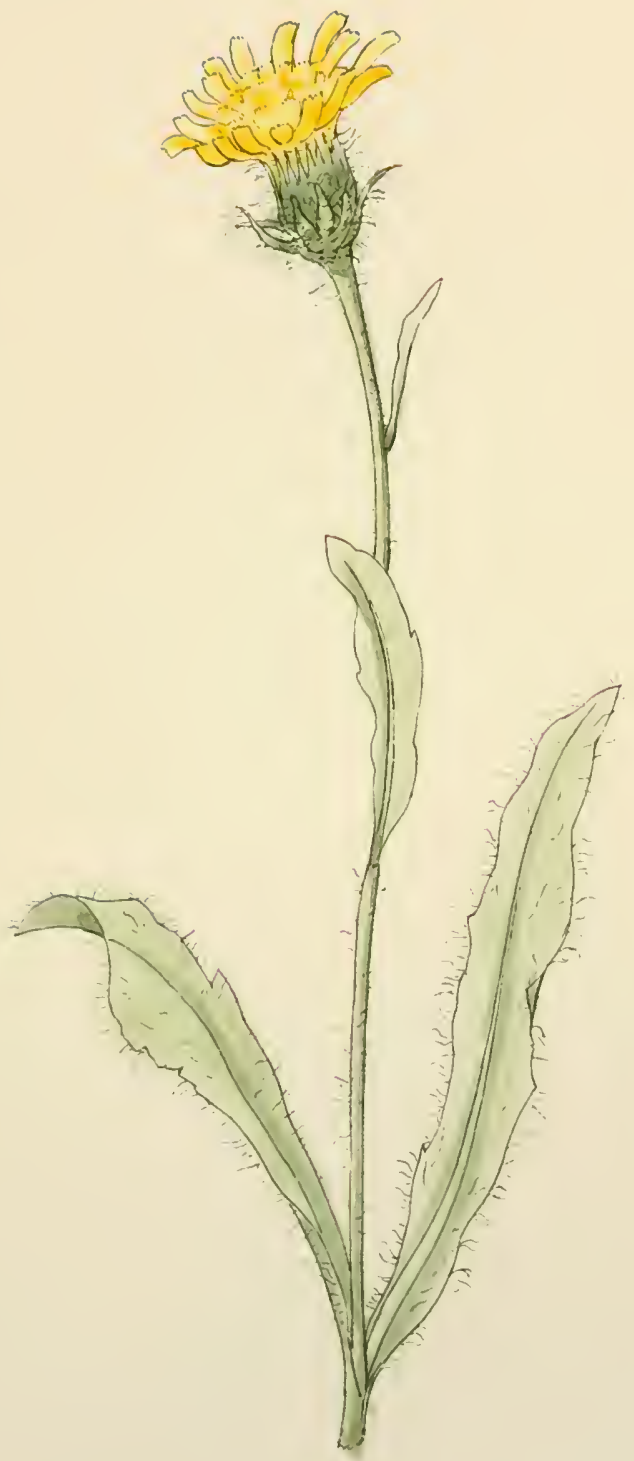

Hieracium scorzonerifolium. 


\section{CXXXIX.}

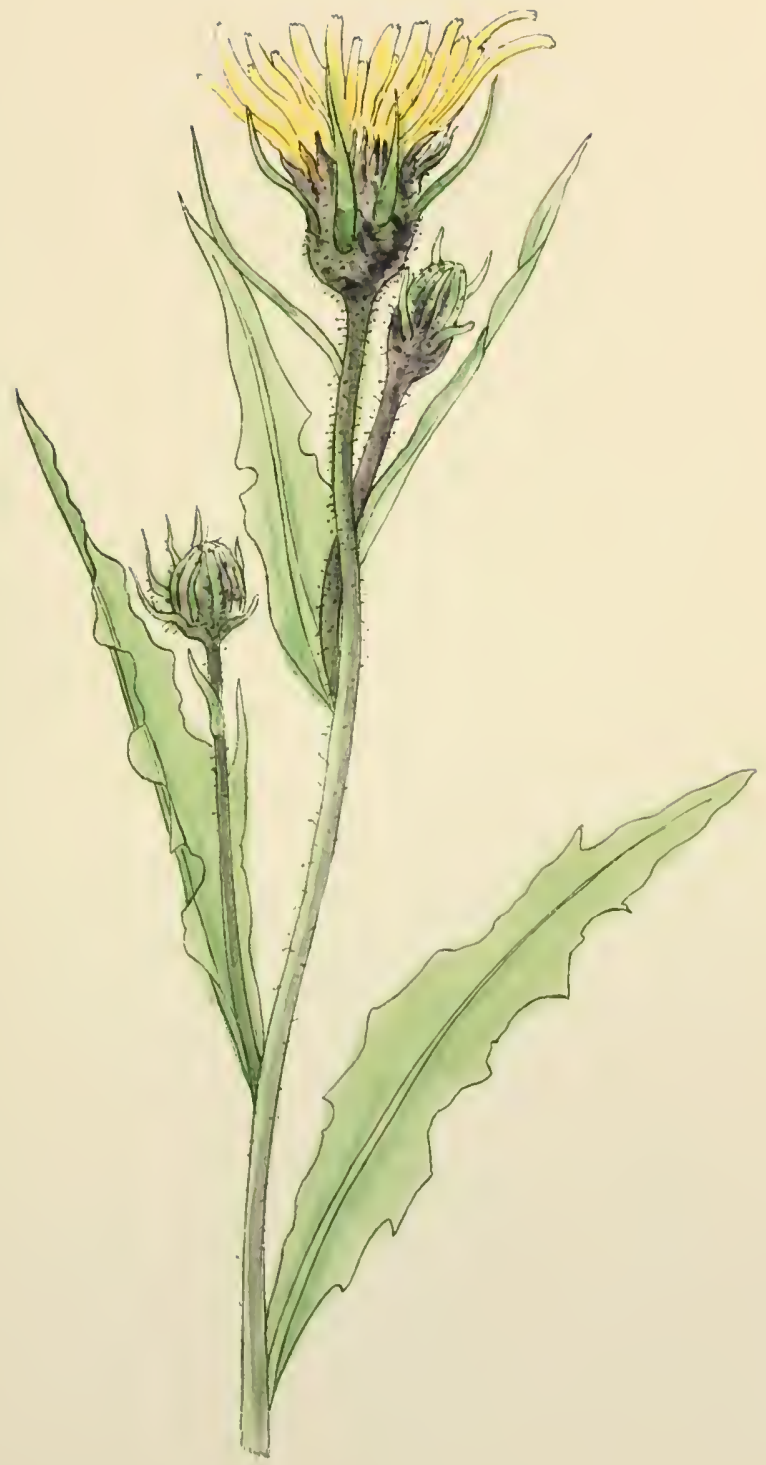

Hieracium albidum. 



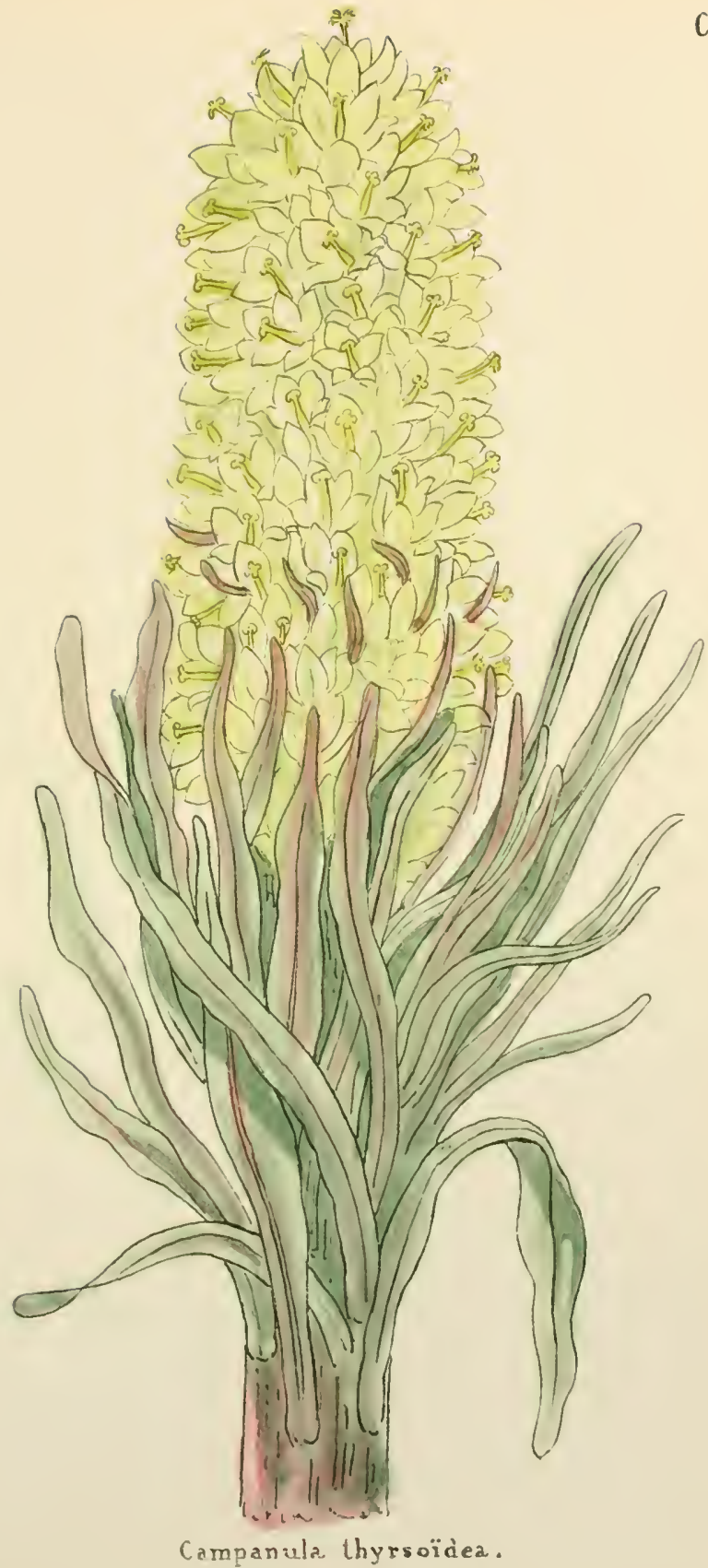



CXLI.

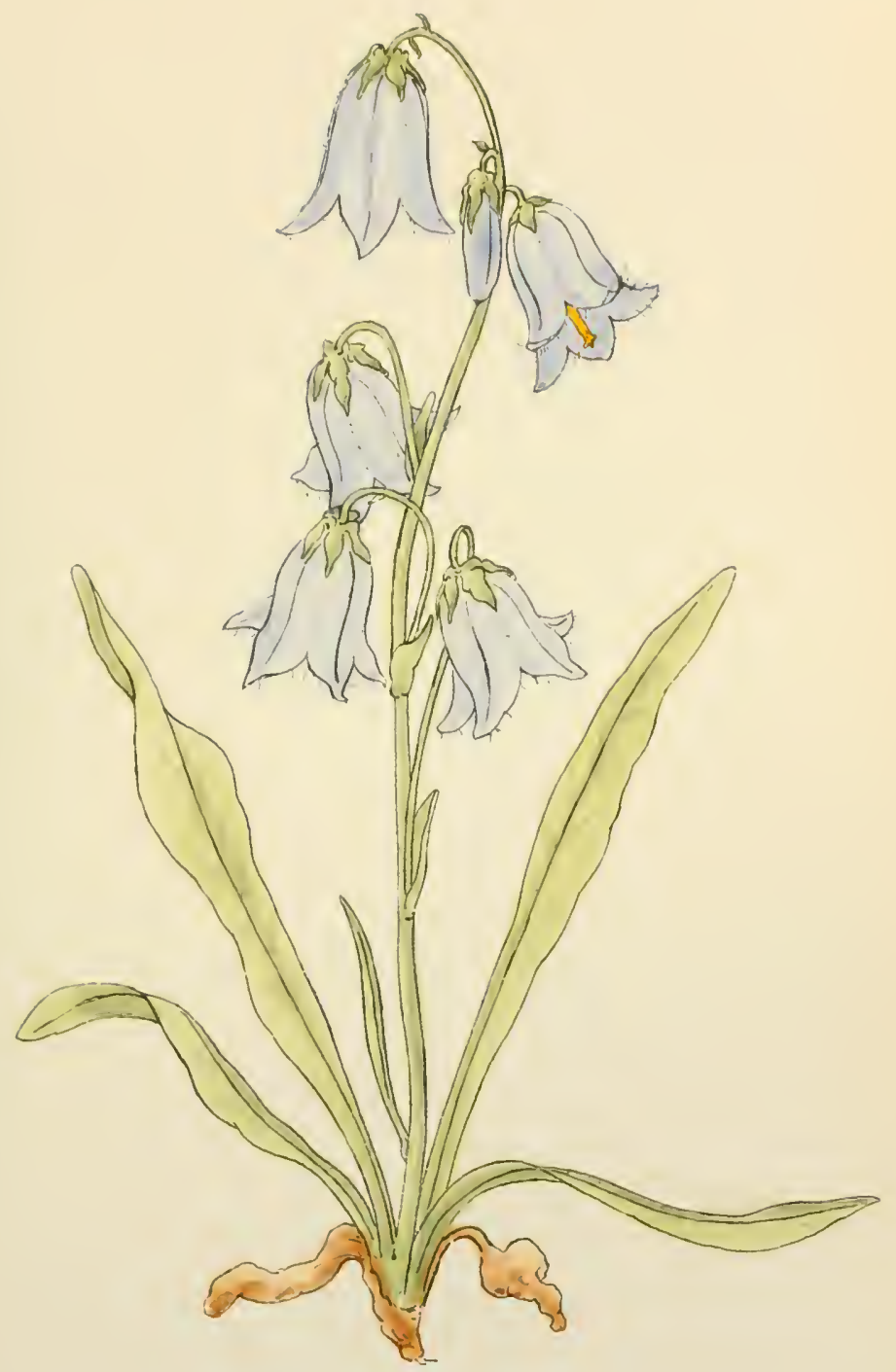

Campanula barbata. 



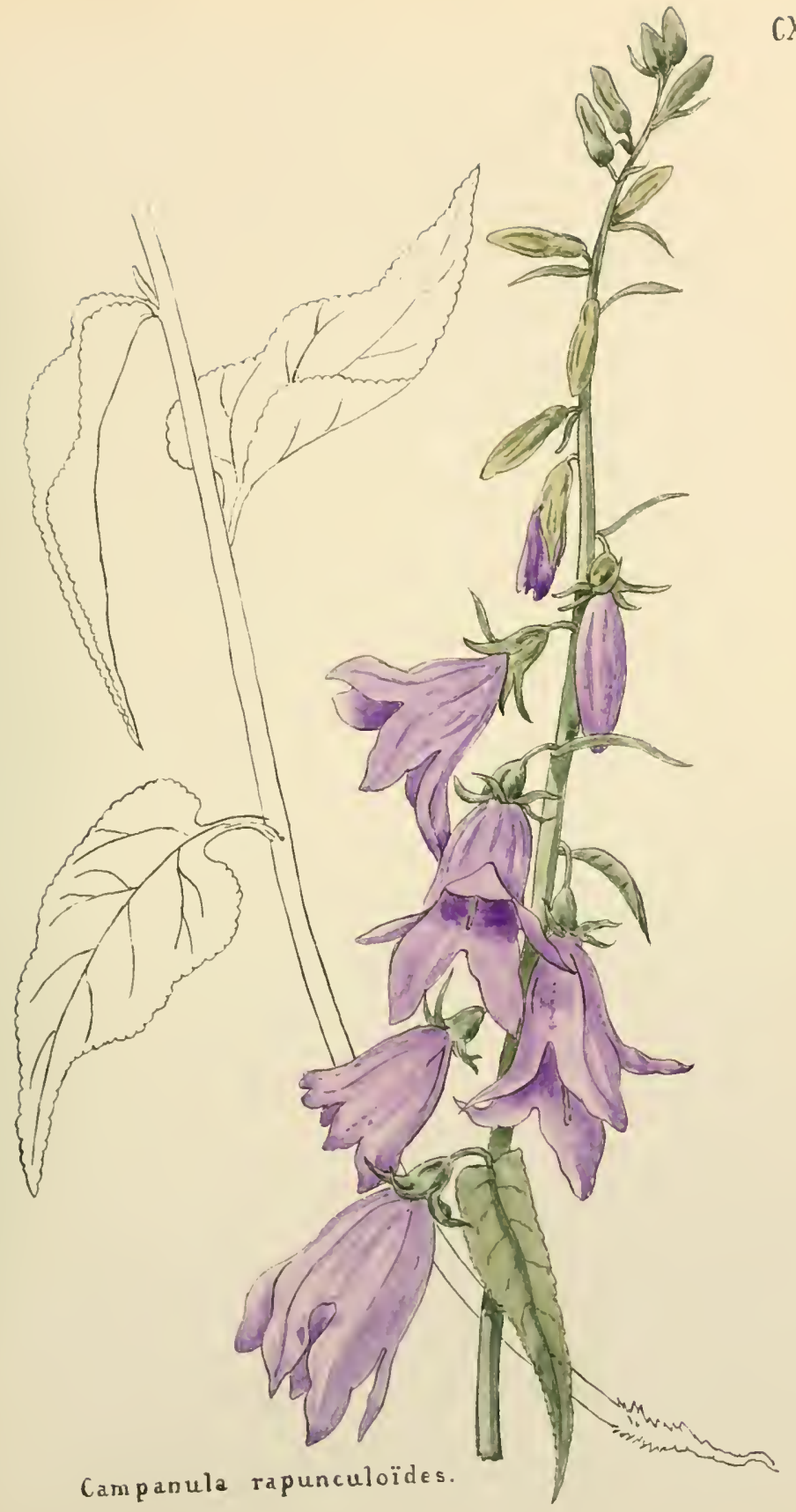



CXLIII.
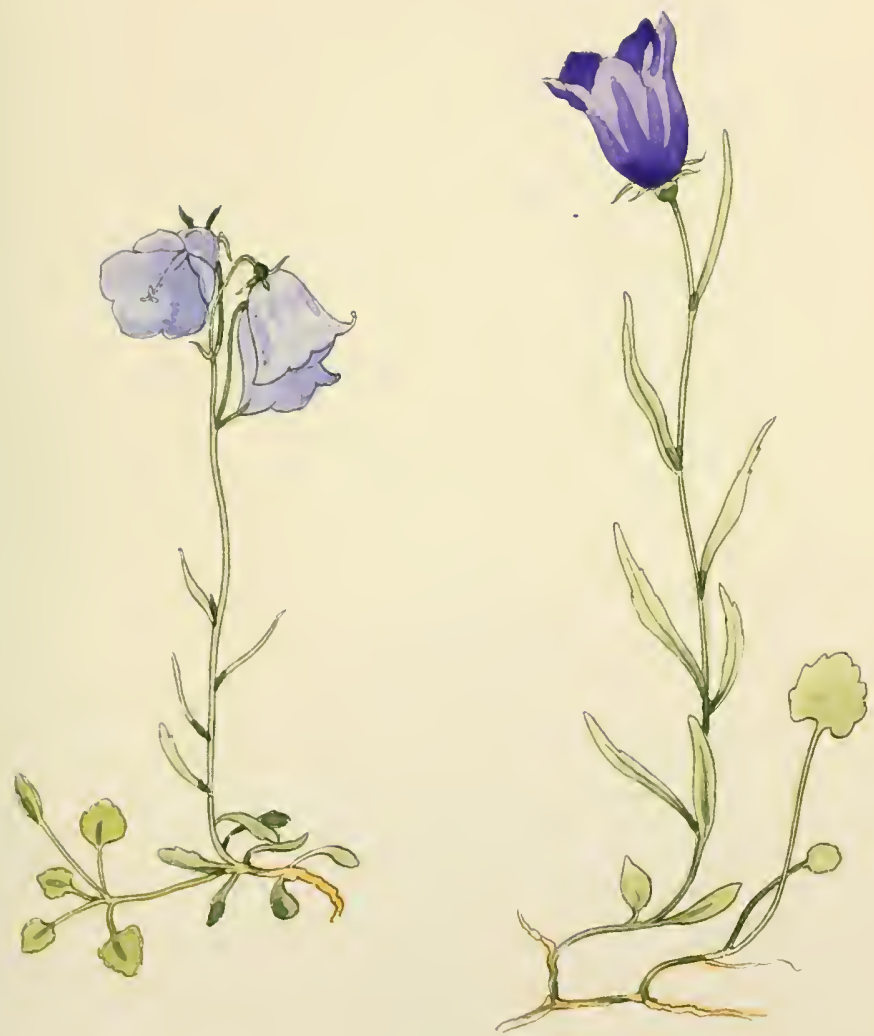

Campanula pusilla. Campanula Scheuchzeri. 

CXLIV.
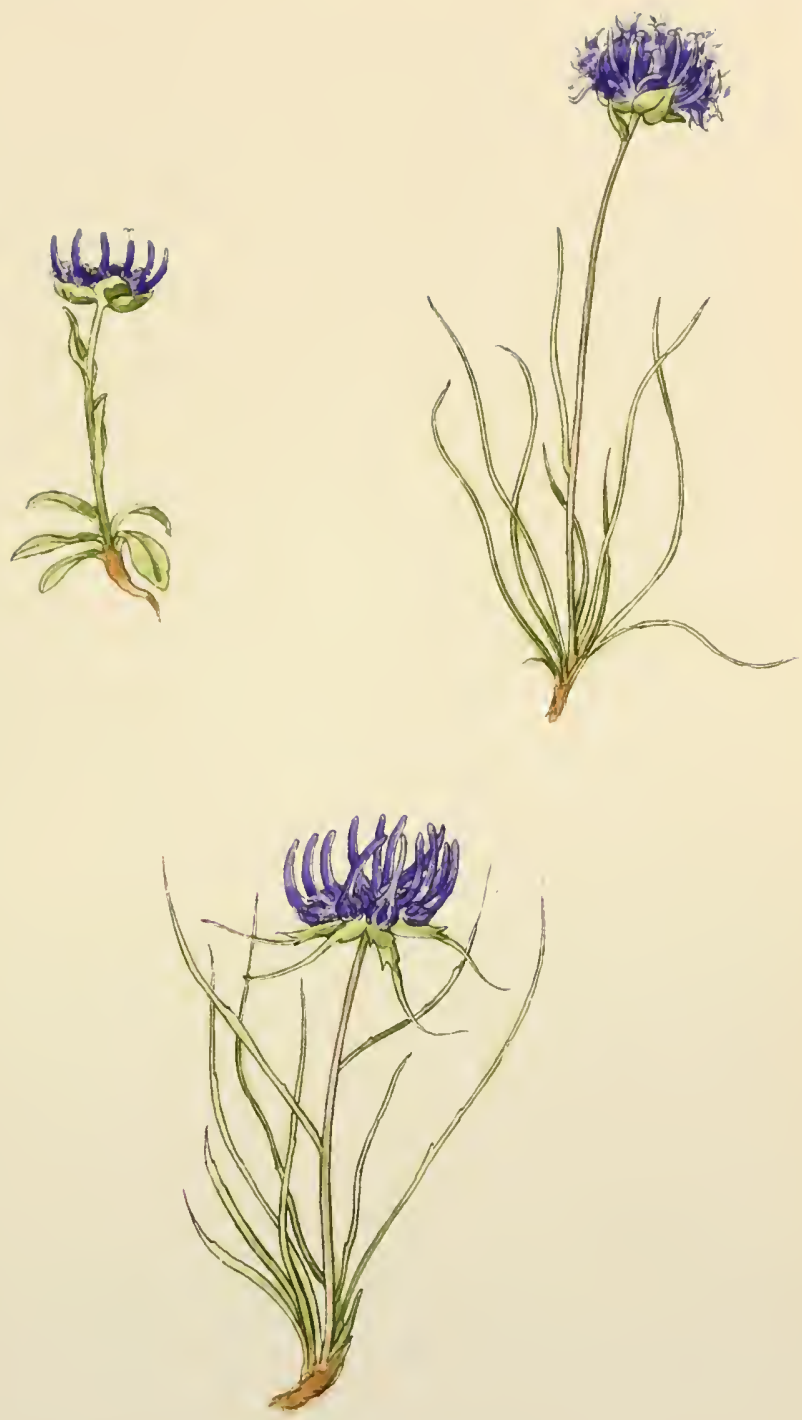

Phyteuma pauciflorum.

Phyteuma hemisphæricum.

Phyteuma humile. 

CXLV.

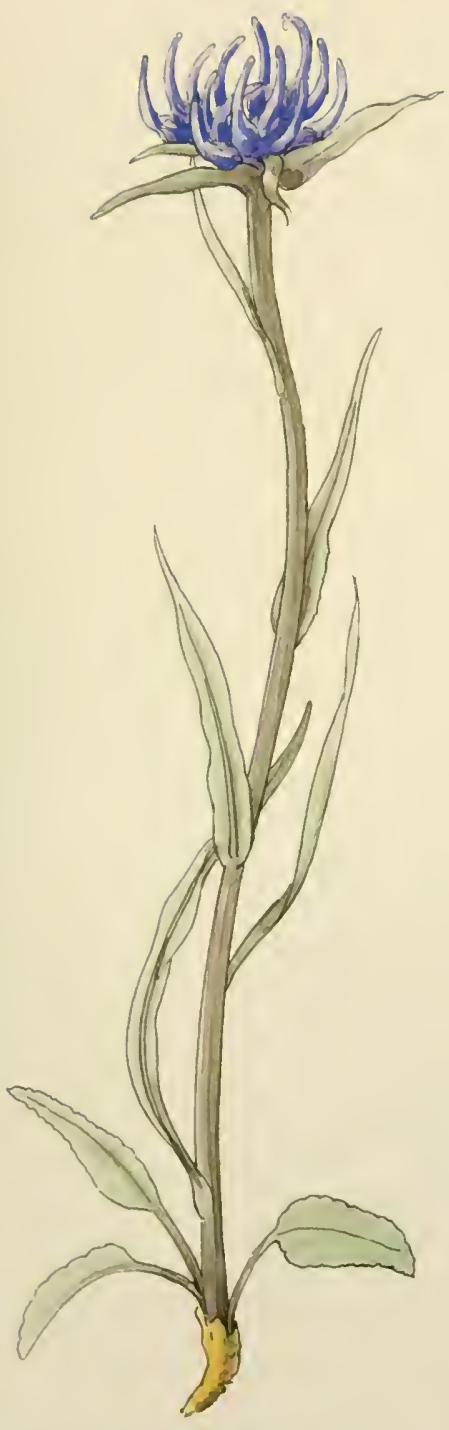

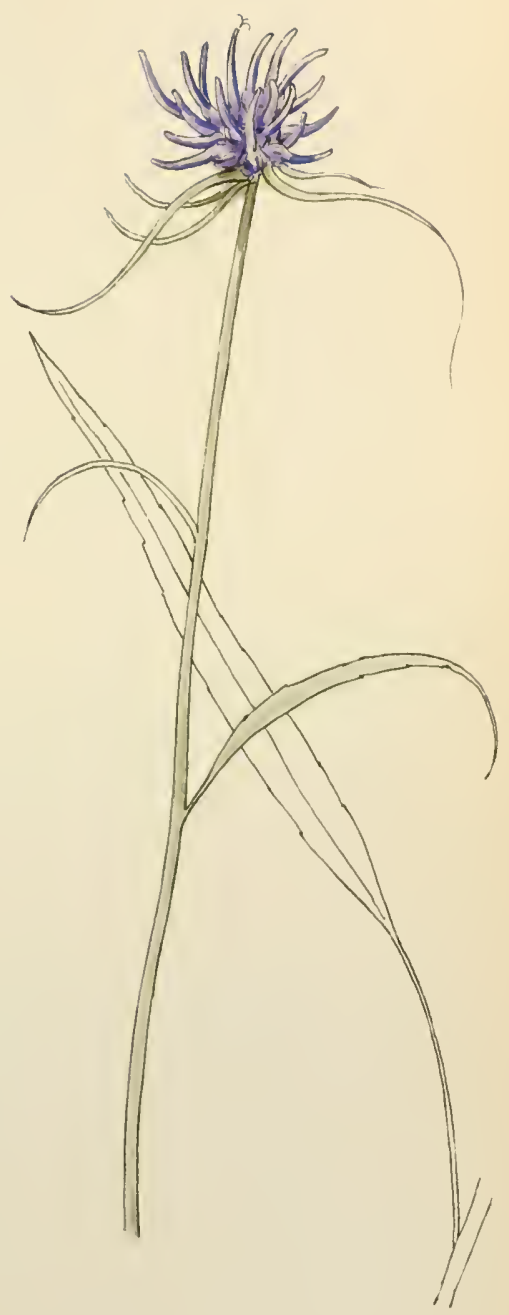

Phyteuma Scheuchzeri.

Phyteuma orbiculare. 

CXLVI.

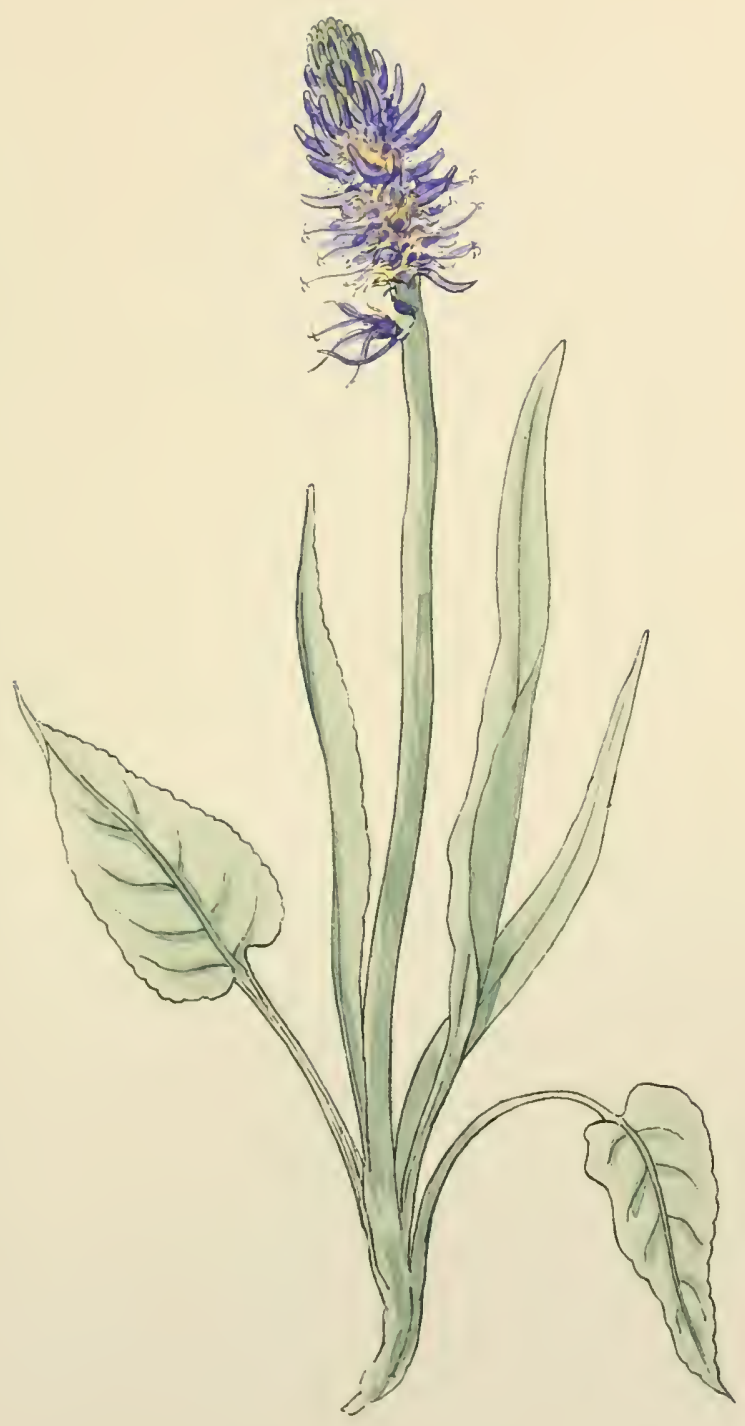

Phyteuma betonicifolium. 



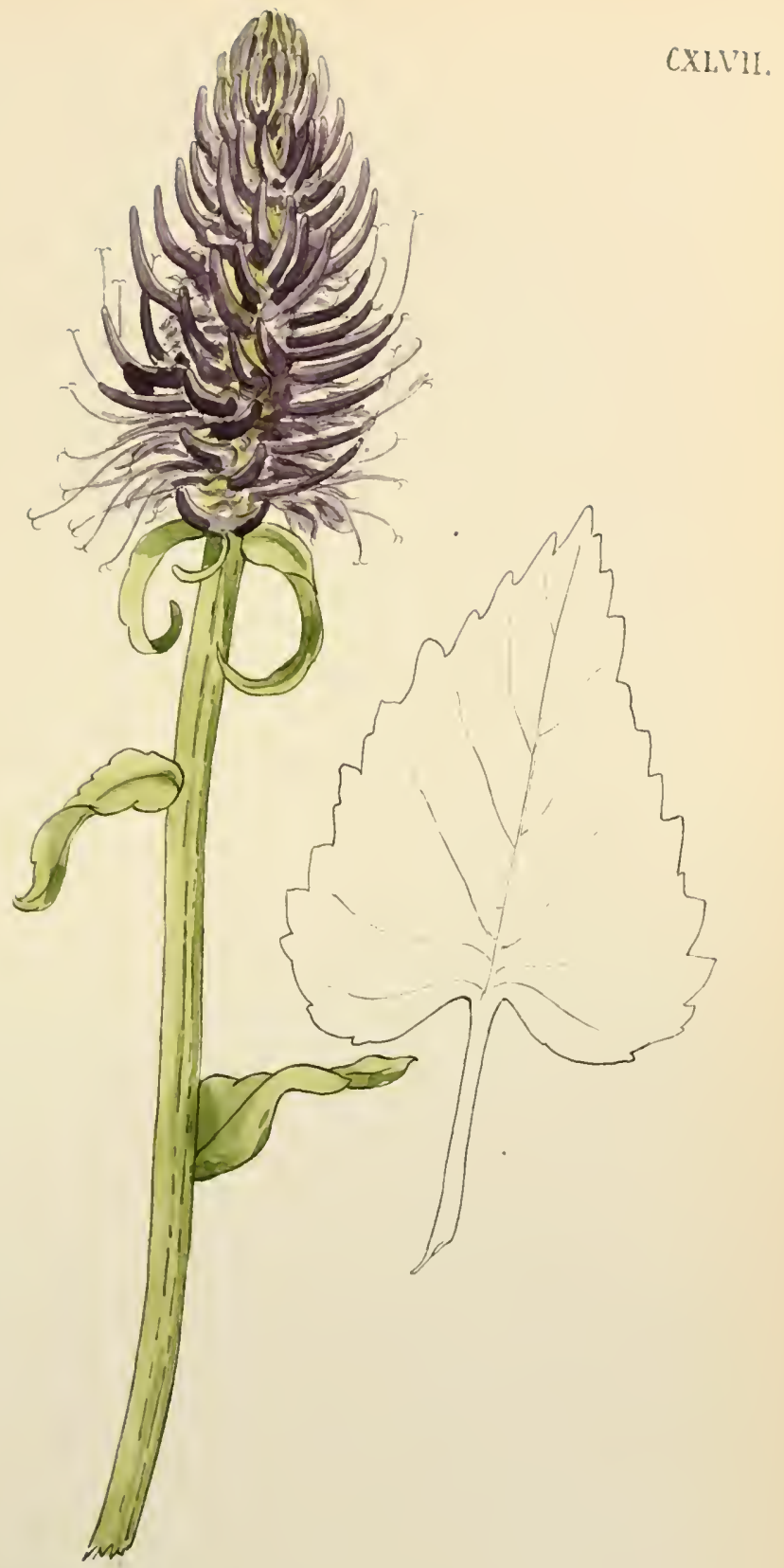

Phyteuma spicatum var. nigrum. 

CXLVIII.
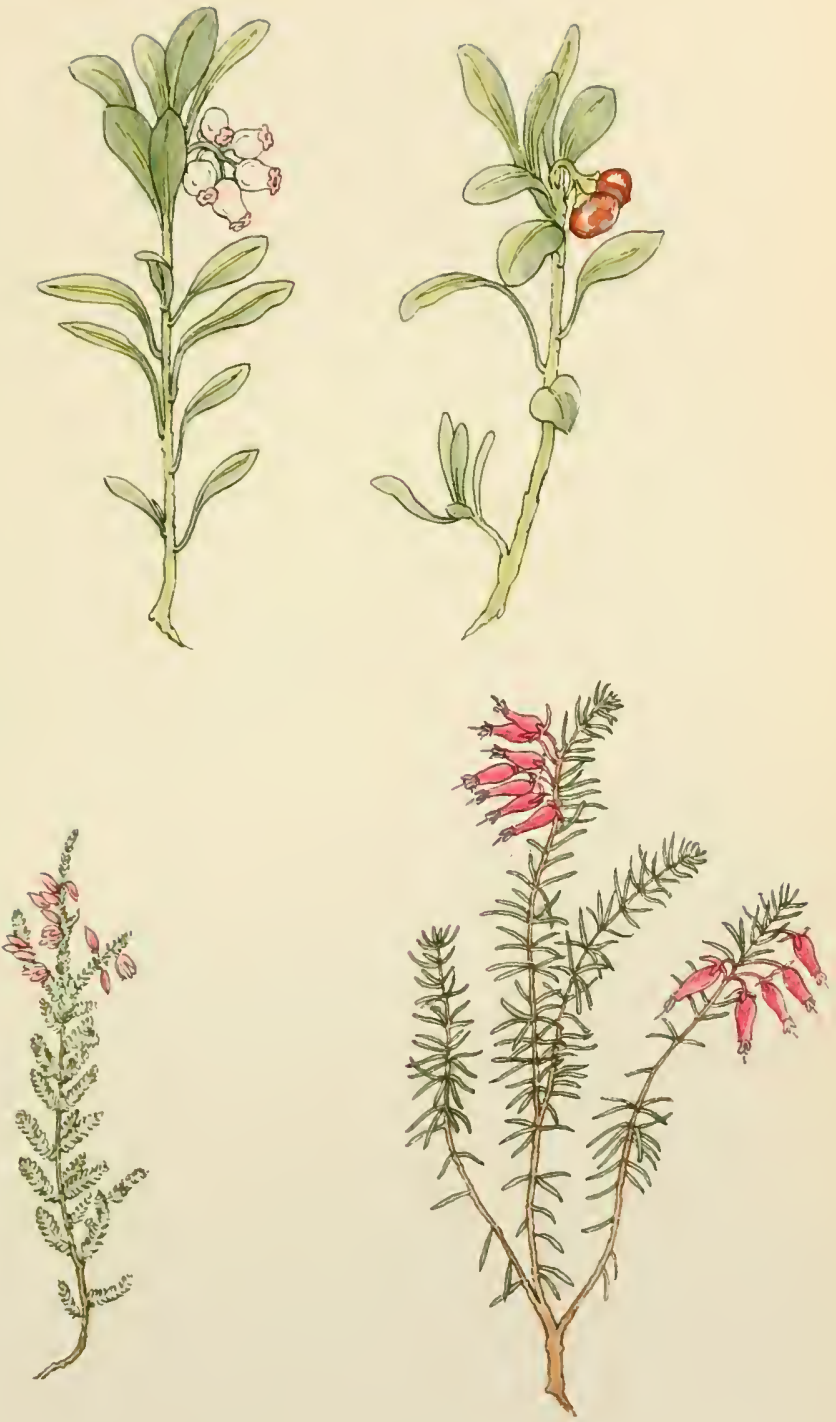

Arctostaphylos officinalis.

Calluna vulgaris.

Erica carnea. 


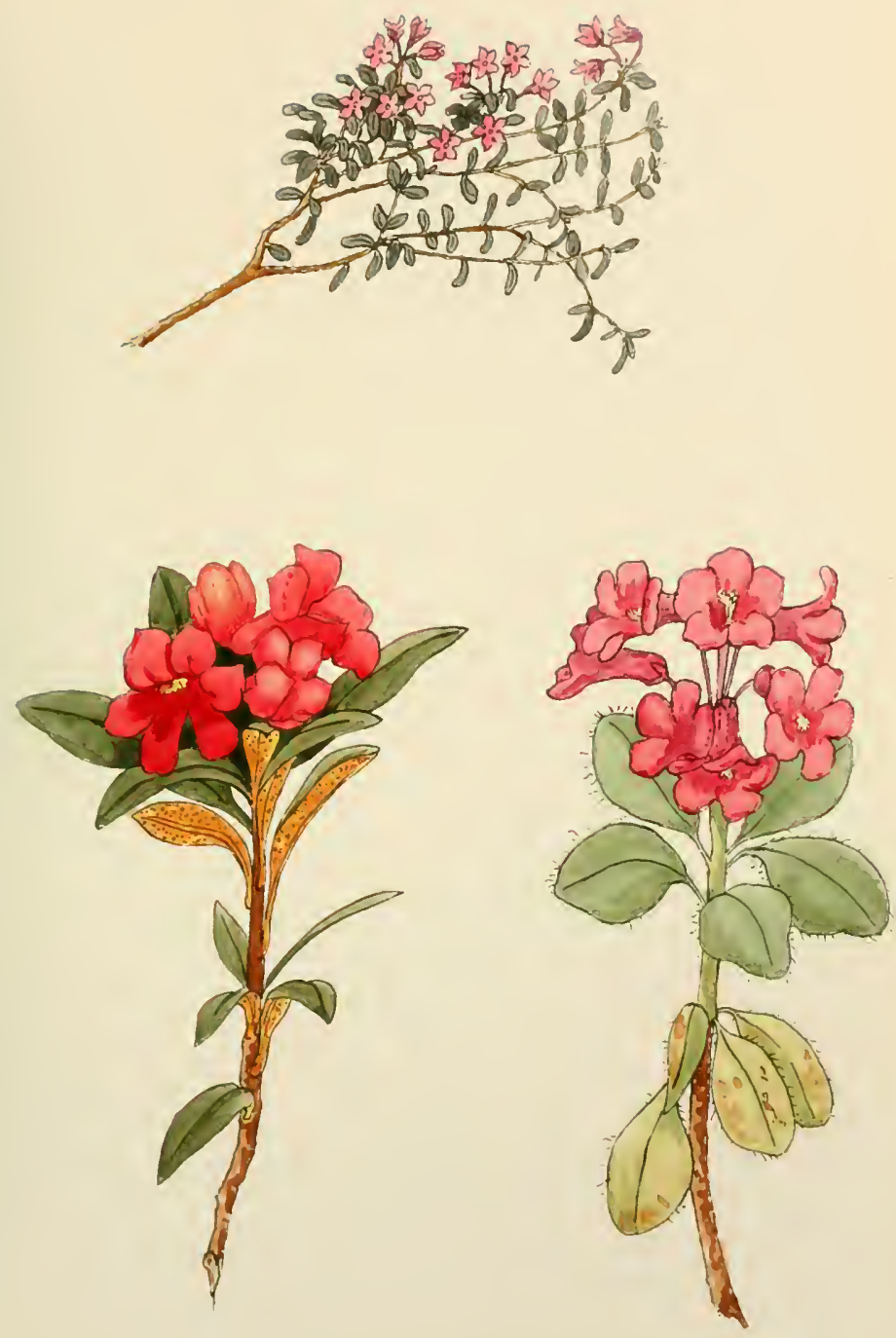

Azalea procumbens.

Rhododendron ferrugineum. Rhododendron hirsutum. 

CL.

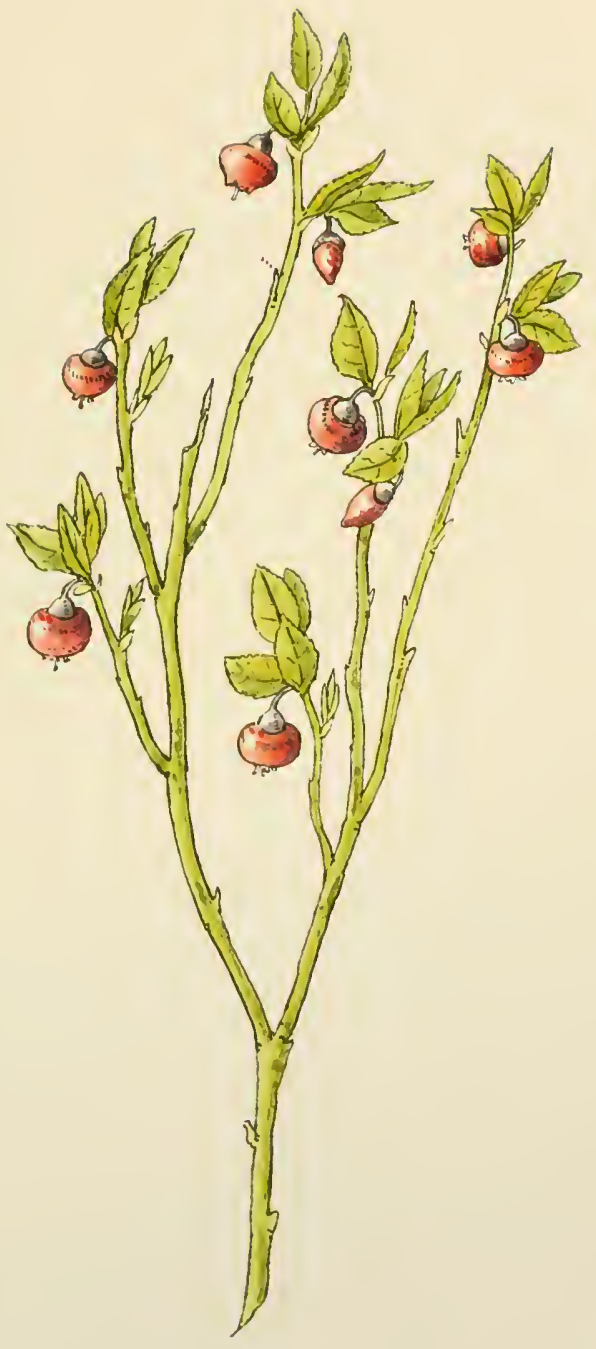

Vaccinium Myrtillus. 


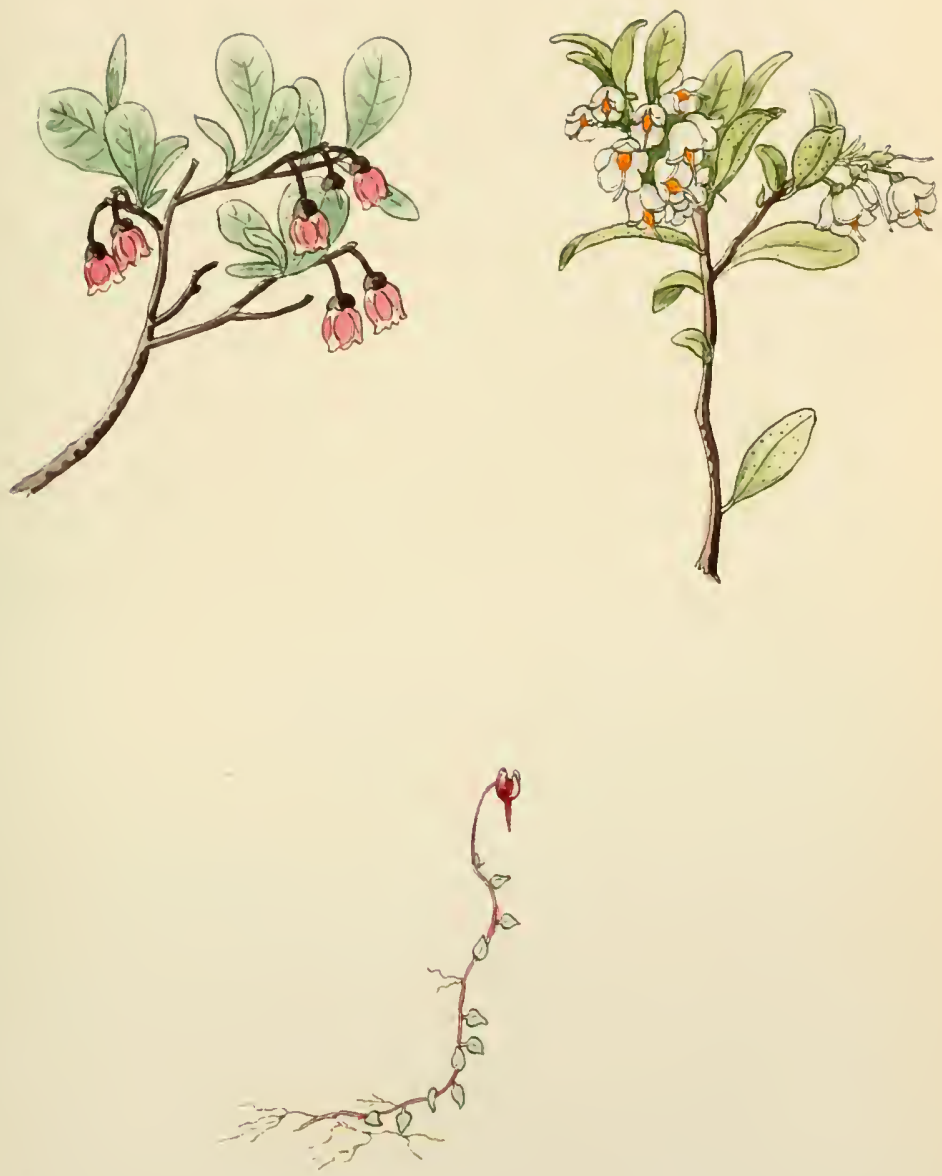

Vaccinium uliginosum.

Vaccinium Vitis-Idæa.

Oxycoccus palustris. 

CLII .

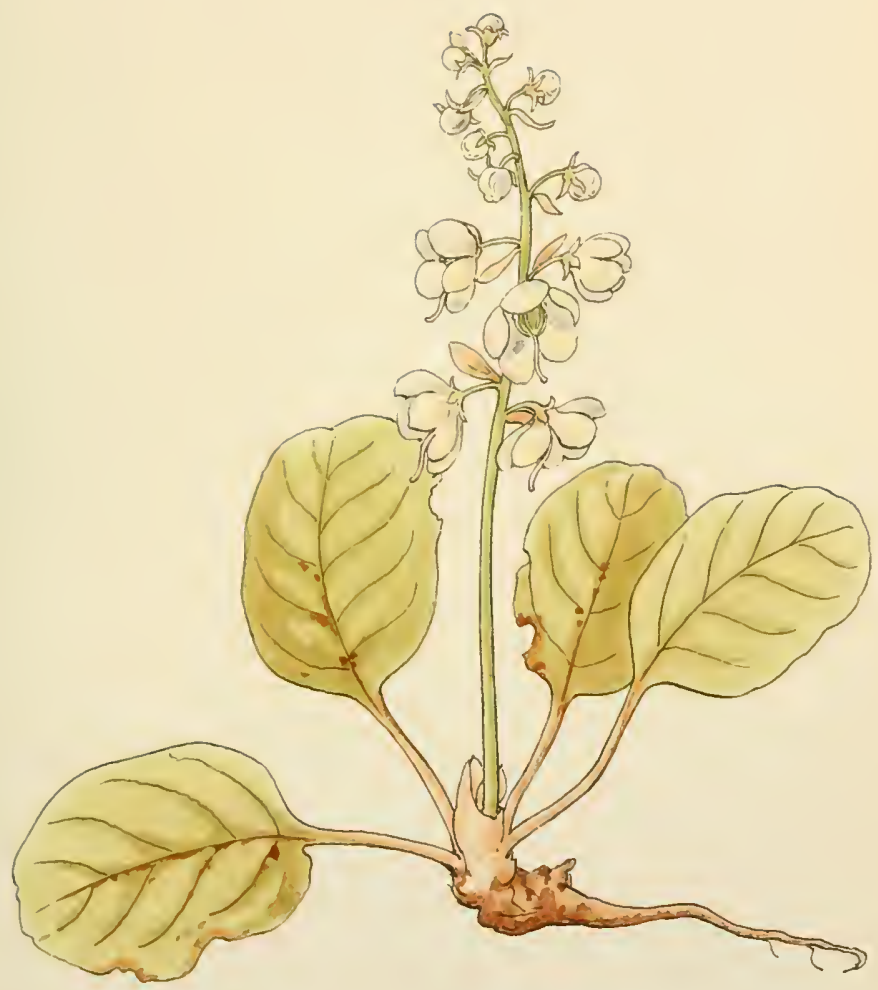

Pyrola rotundifolia. 

CLIII.
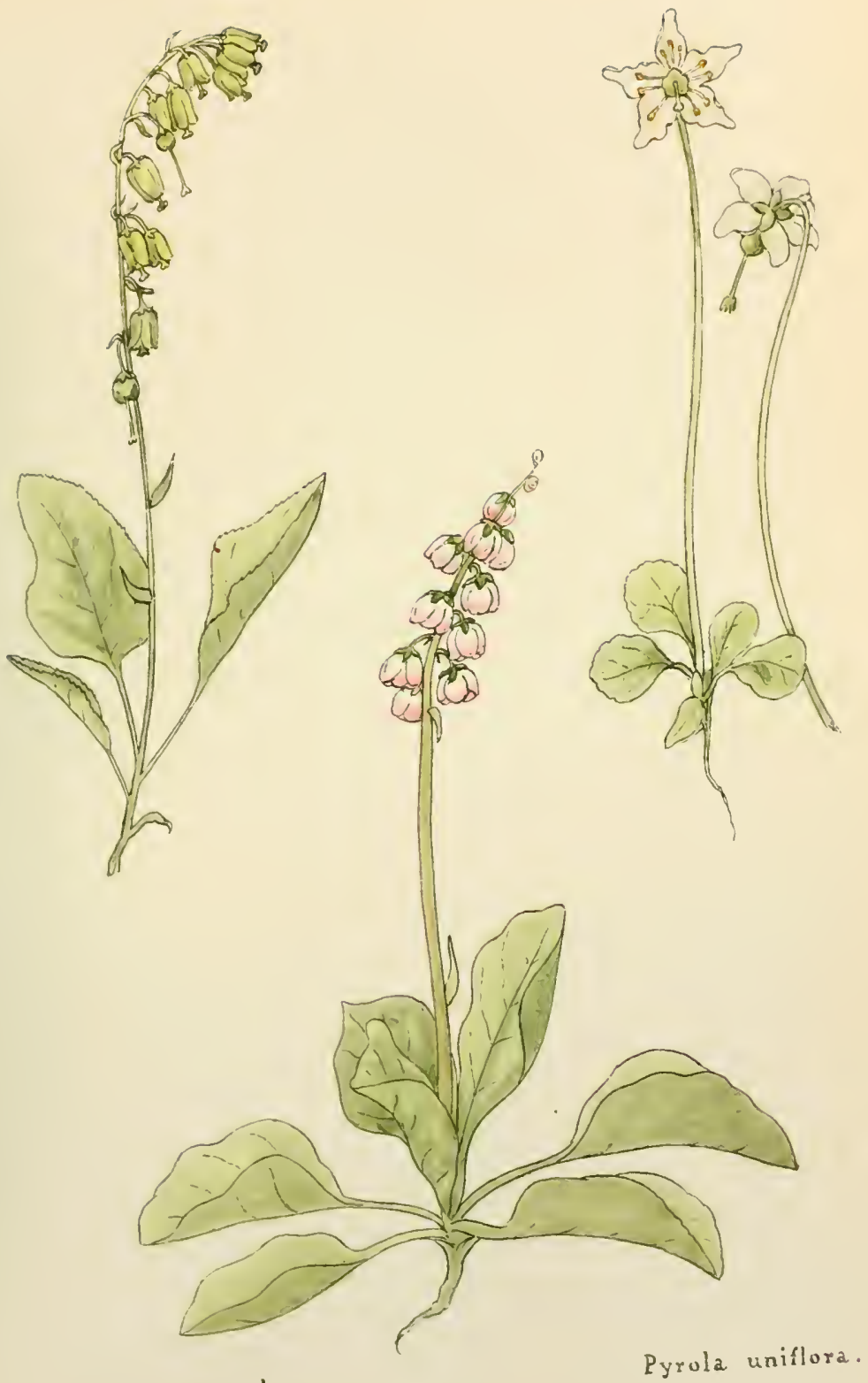

Pyrola secunda.

Pyrola minor. 



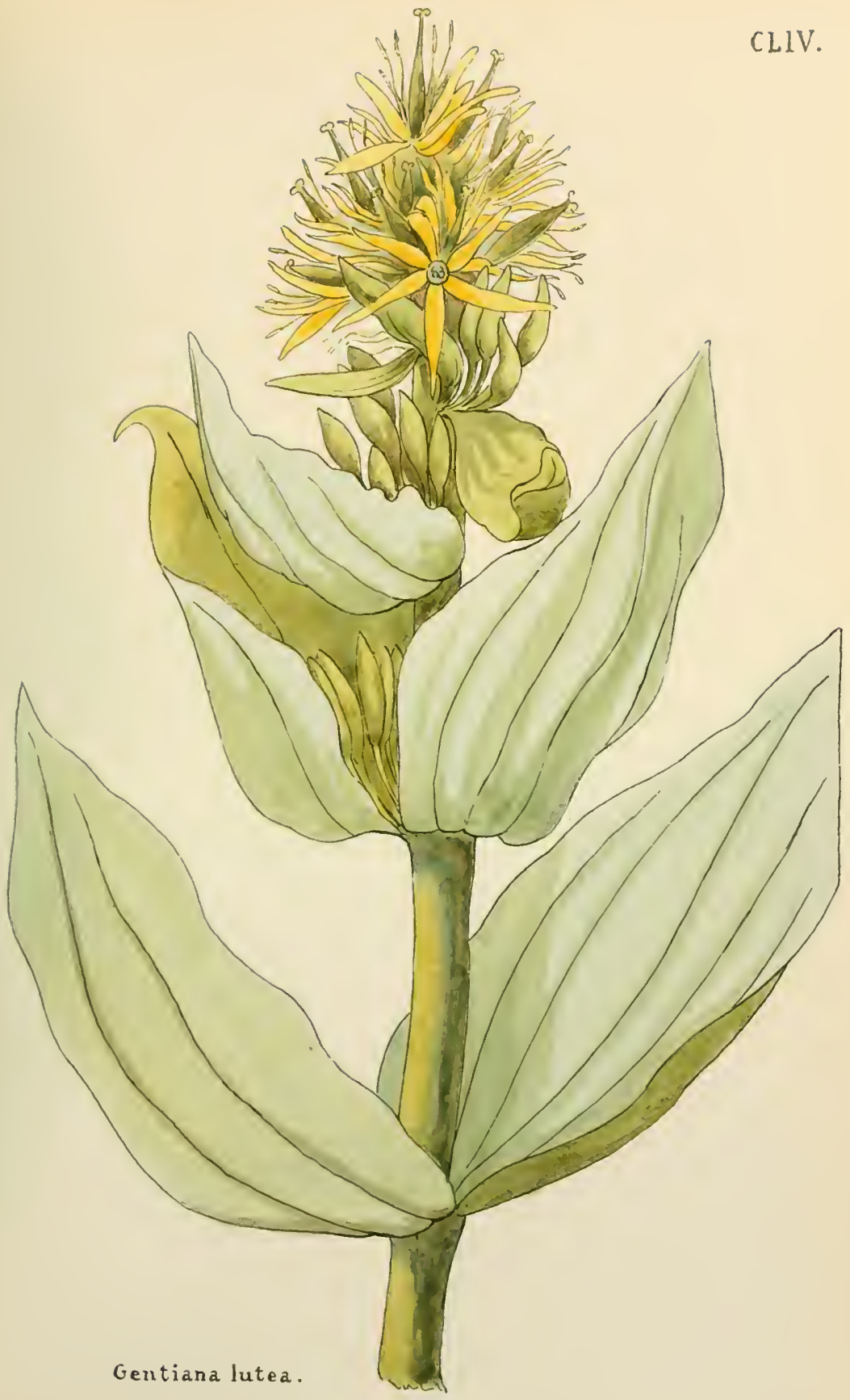


CLV.

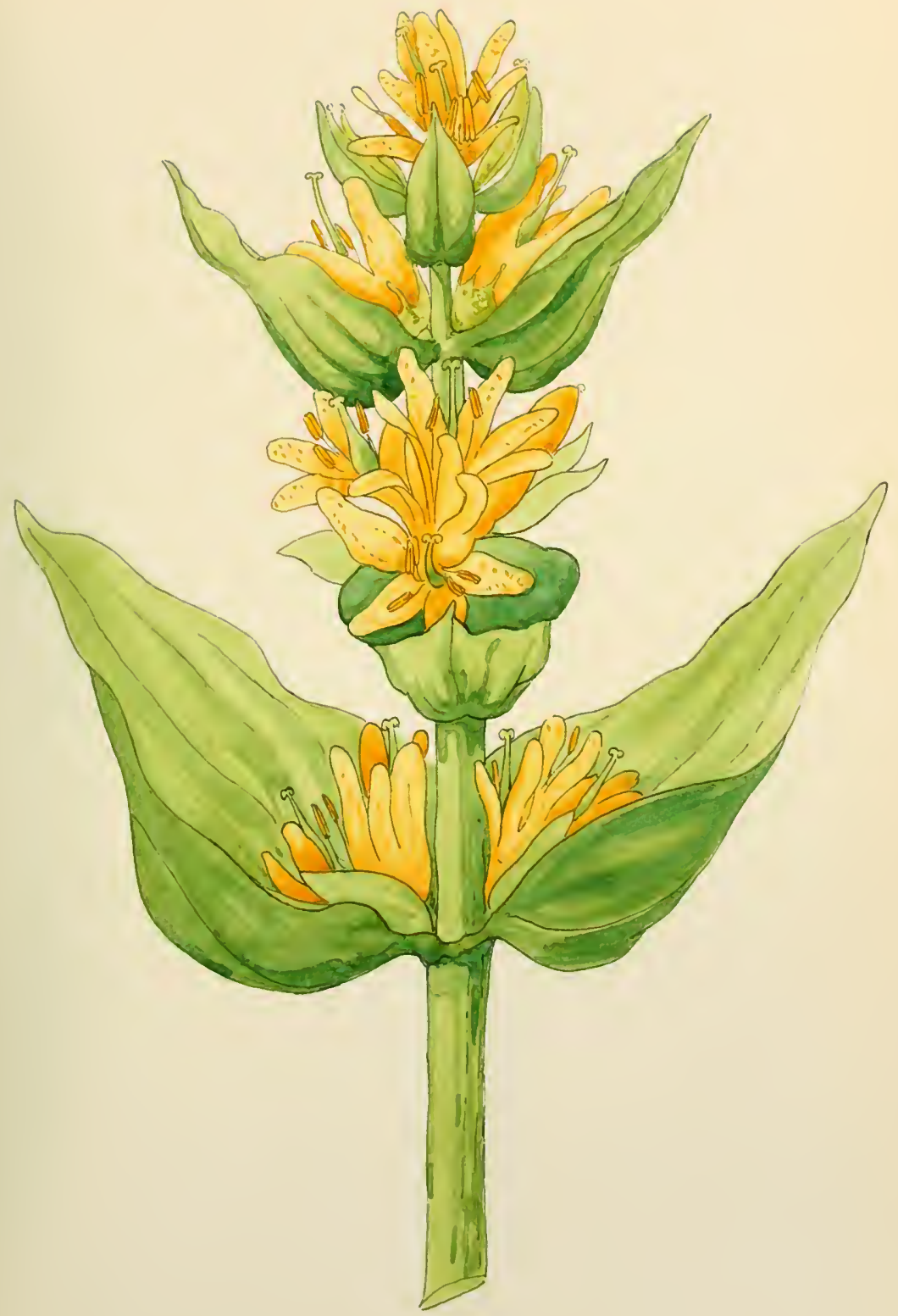

Gentiana Charpentieri. 



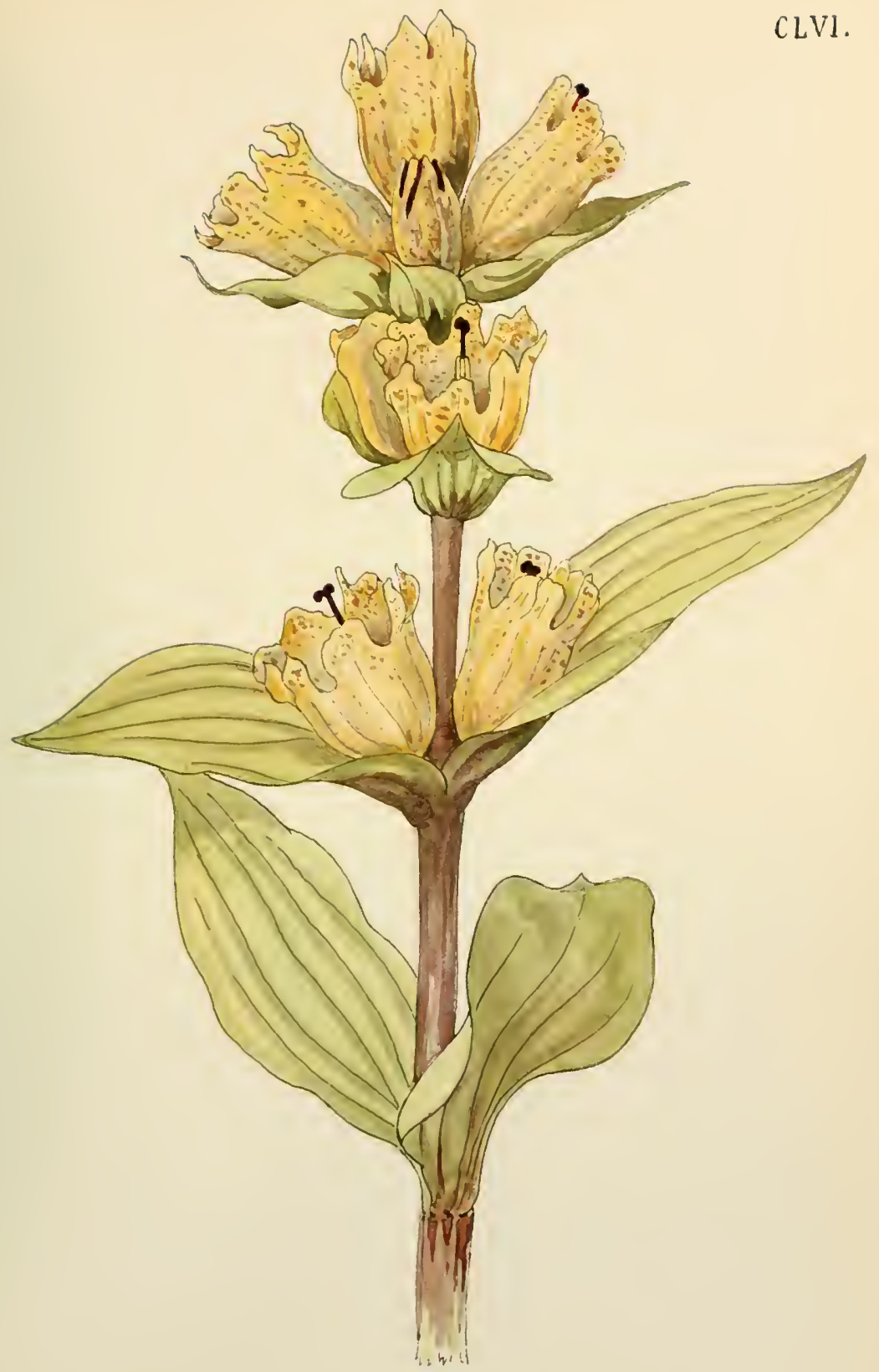

Gentiana punctata. 

CLVII.

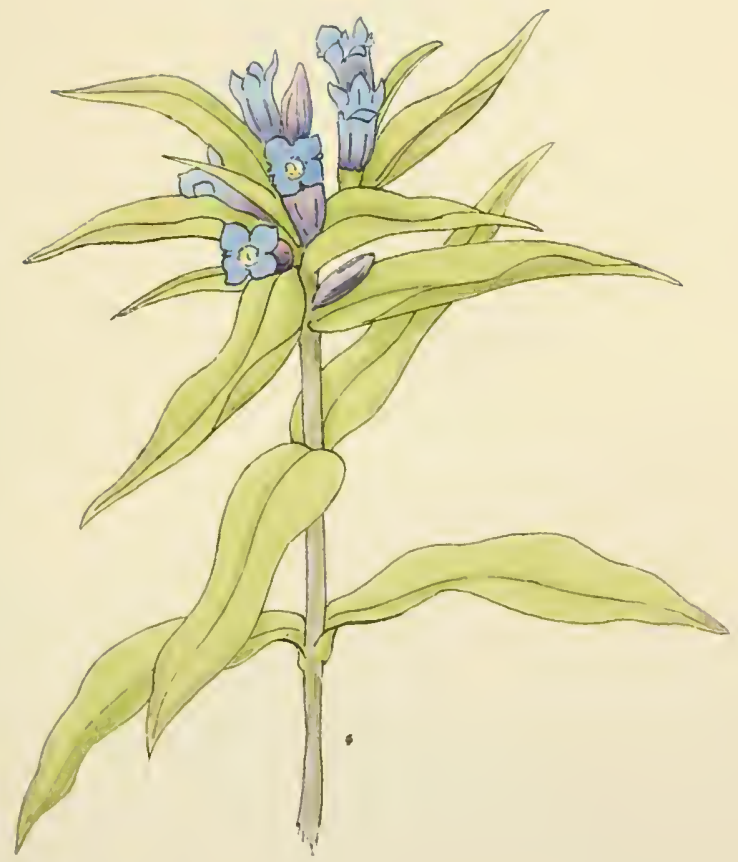

Gentiana cruciata. 



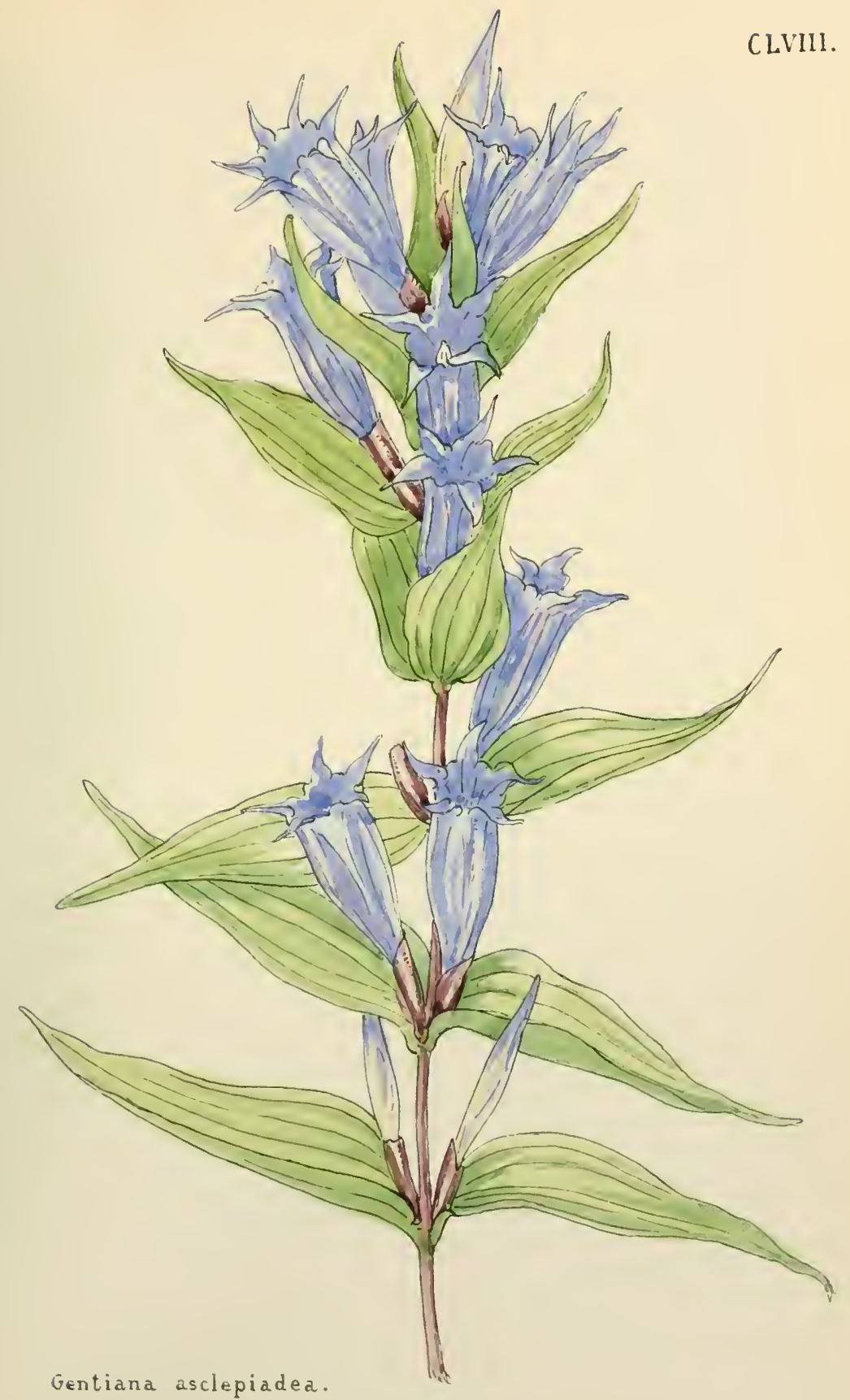




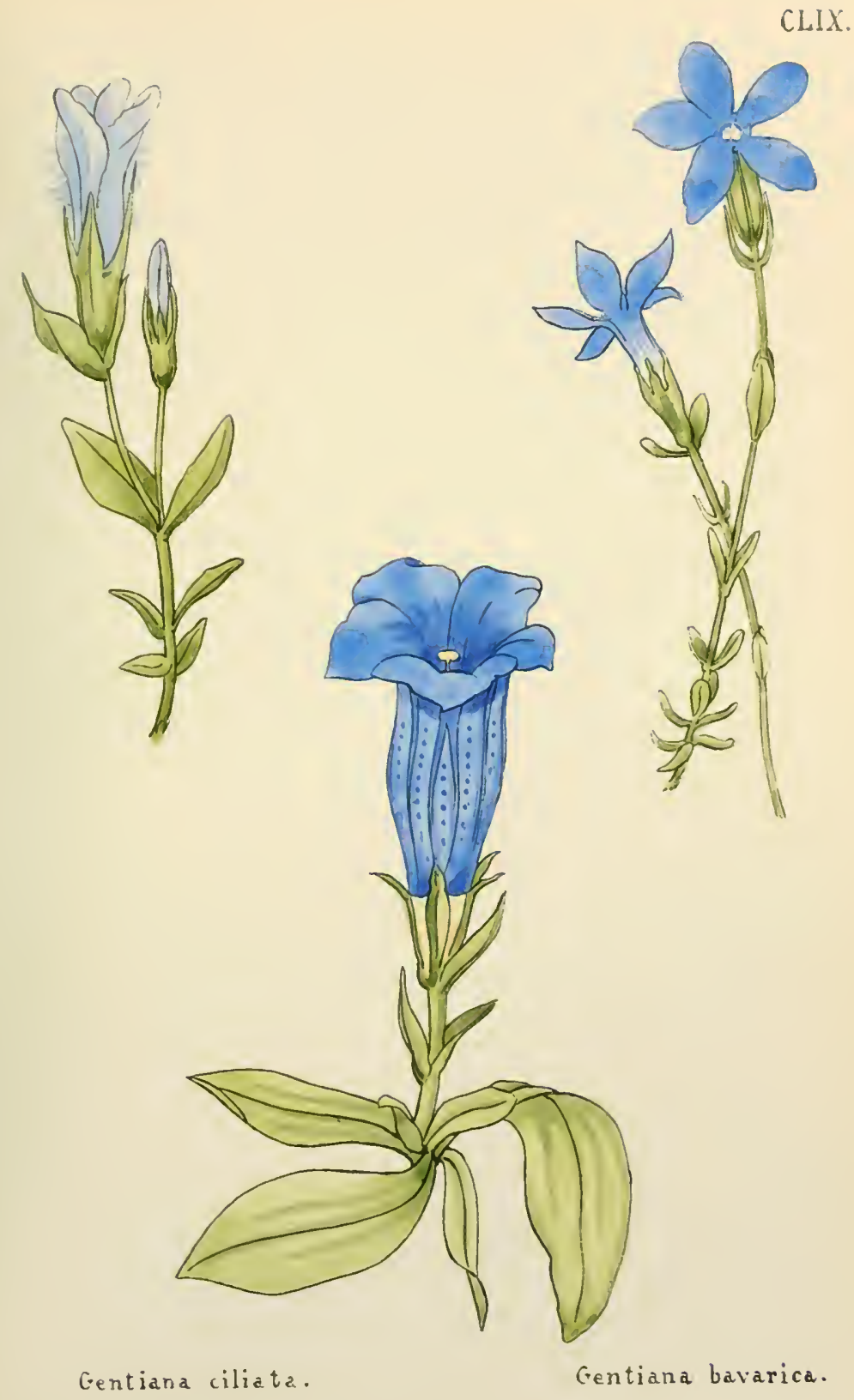

Gentiana excisa. 


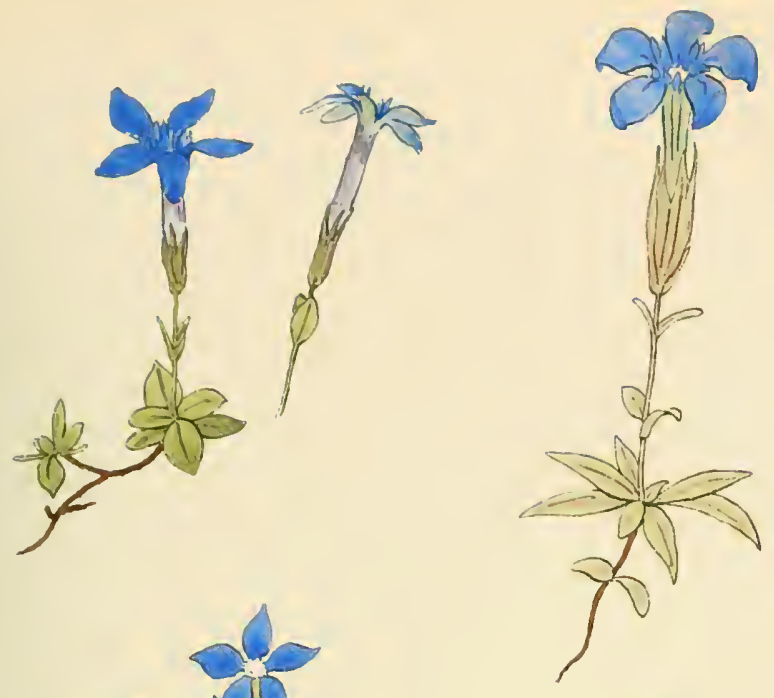

CLX.

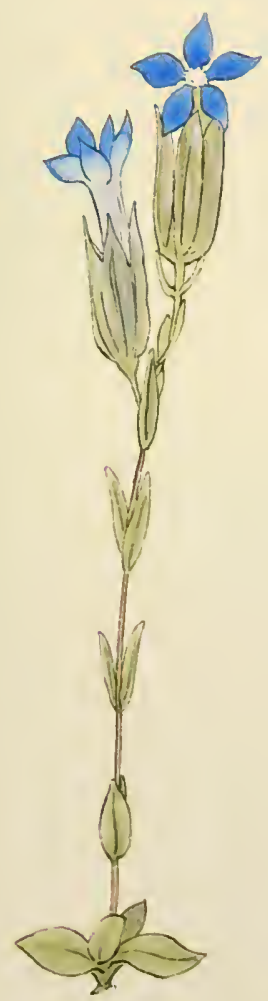

Gentiana brachyphylla.

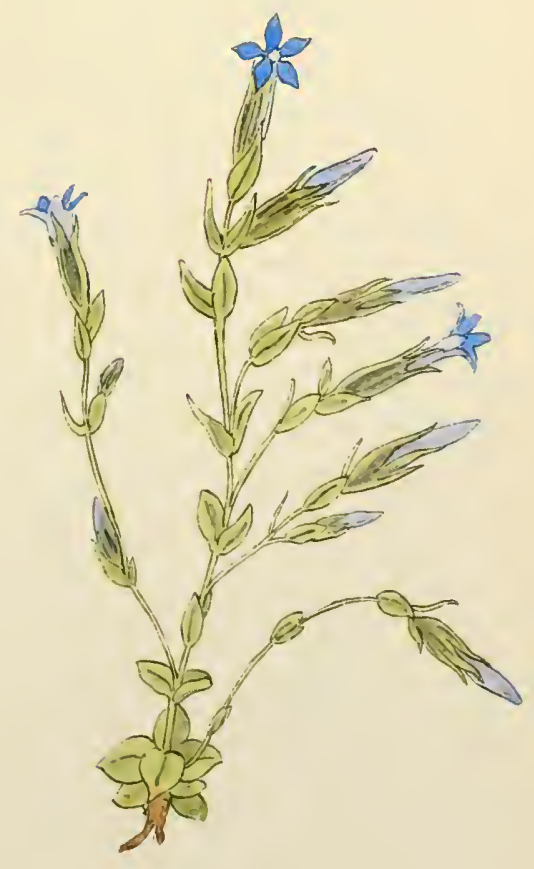

Gentiana verna.

Gentrana utriculosa.

Gentiana nivalis. 


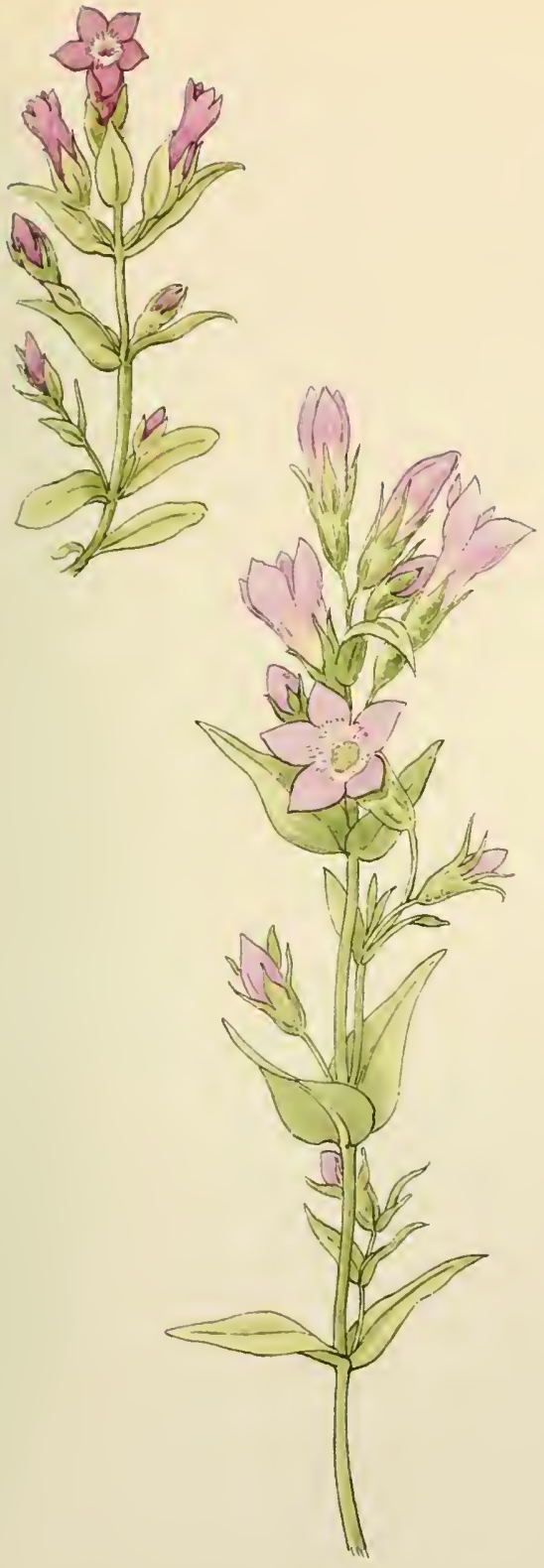

Gentiana obtusifolia.

Gentiana germanica.
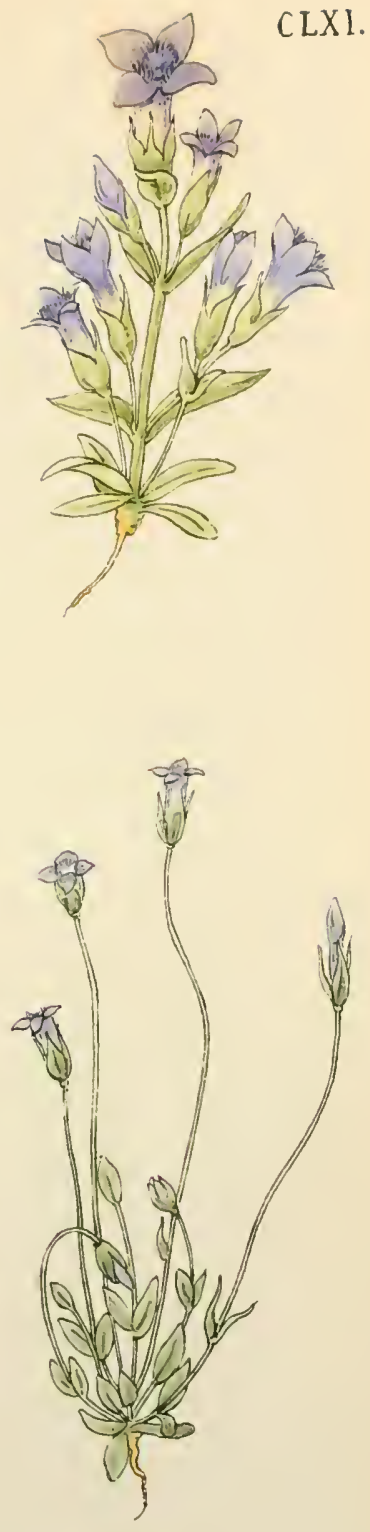

Gentiana campestris.

Gentiana tenella. 
CLXII.
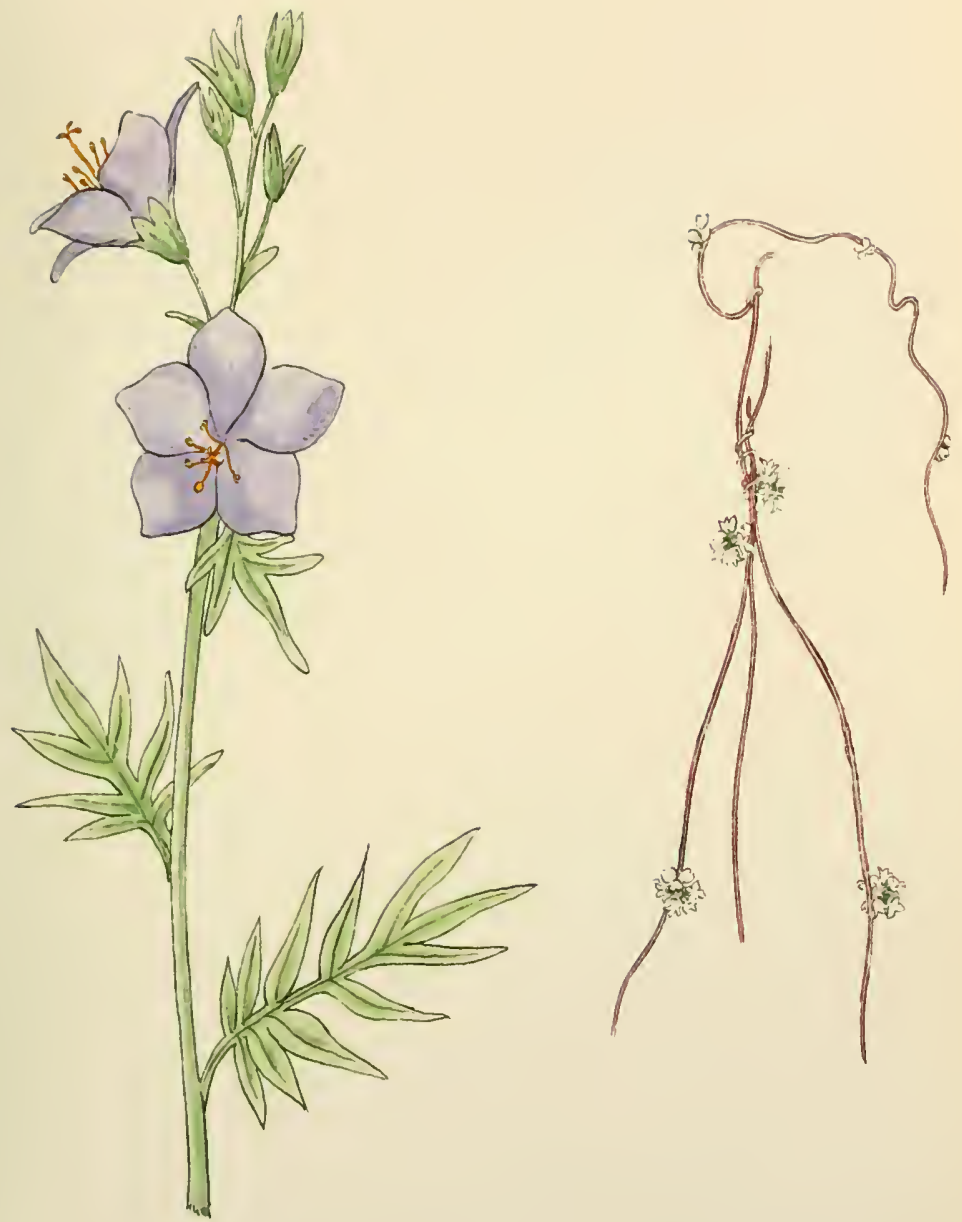

Polemonium creruleum. Cuscuta europias. 


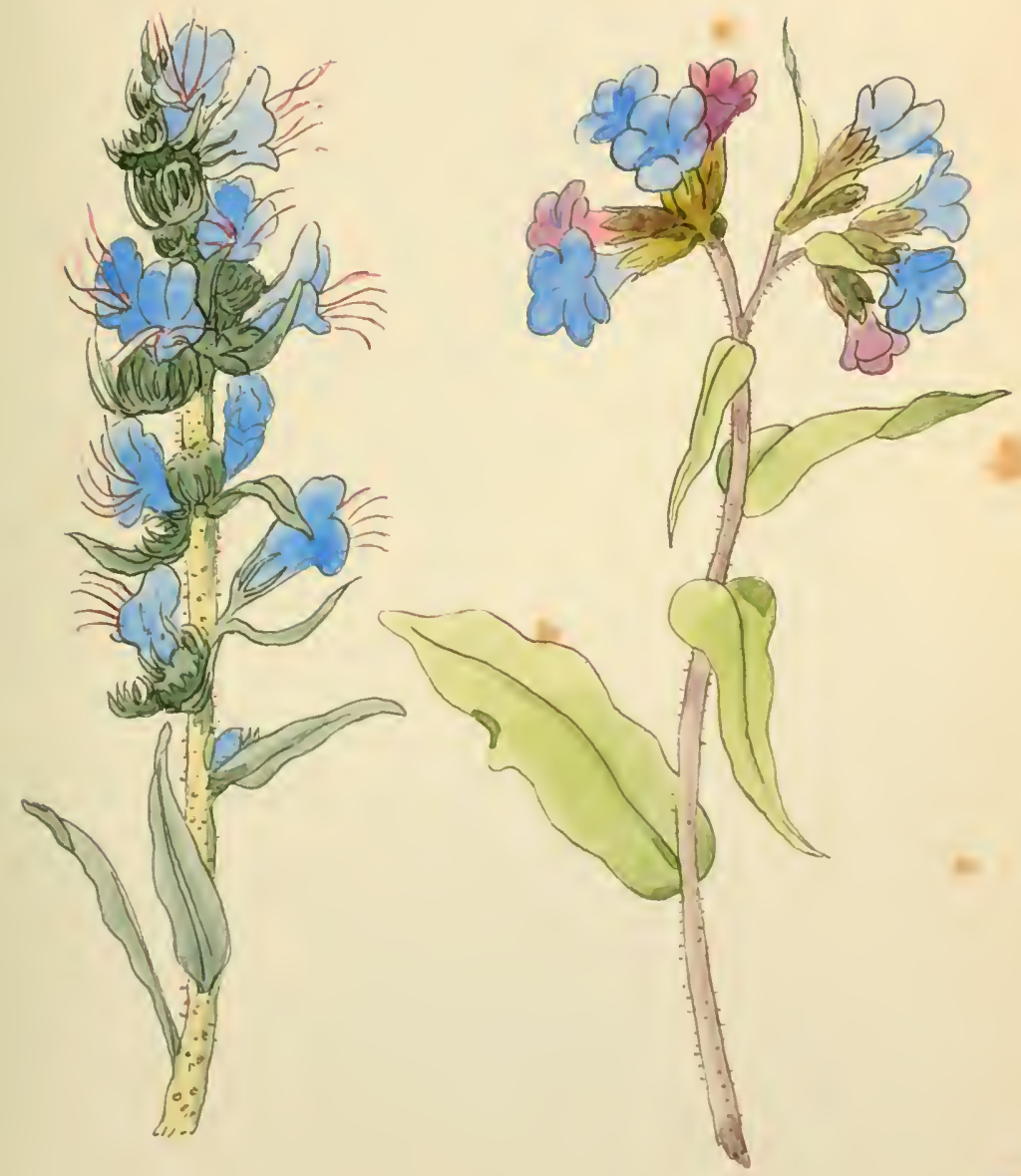

Echium vulgare. 
120

D. 


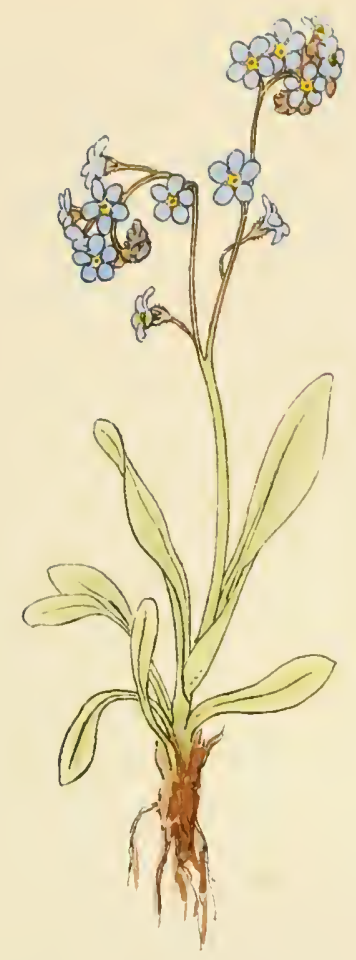

CLXIV.

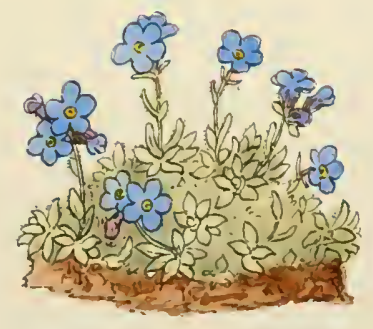

Myosotis alpestris.

Eritrichium nanum. 



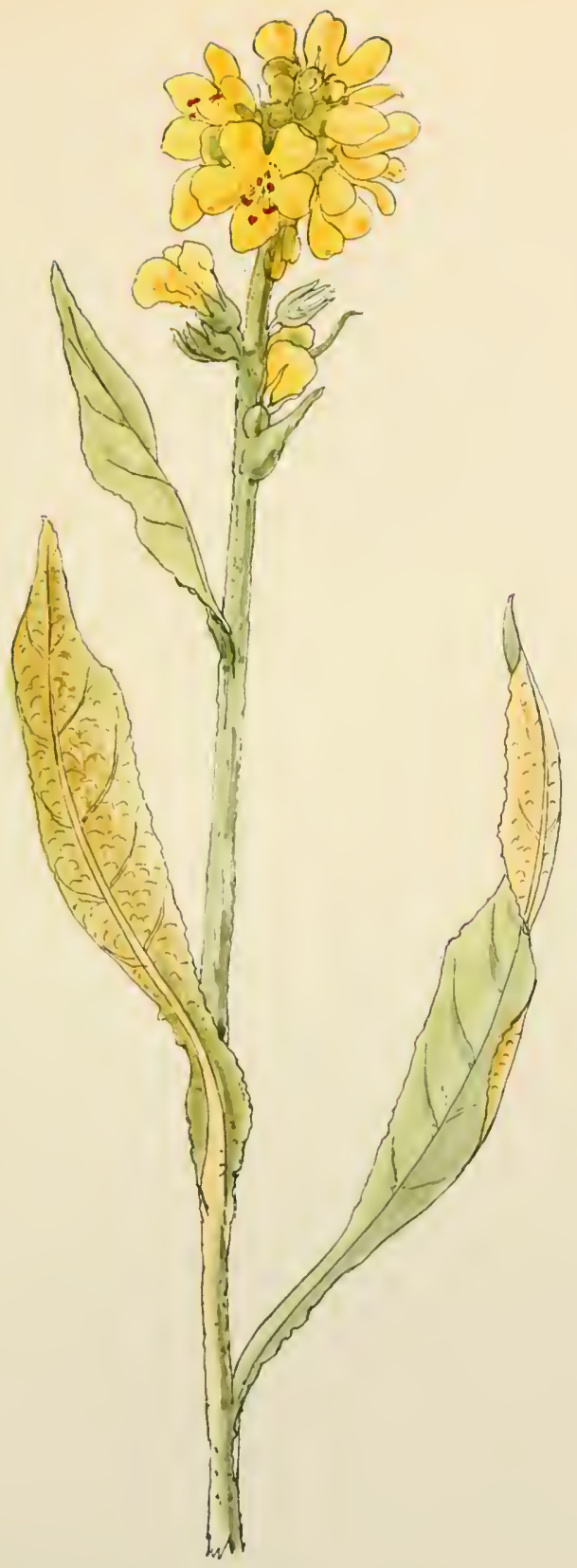

CLXV.

Verbascum pulverulentum. 

CLXVL.
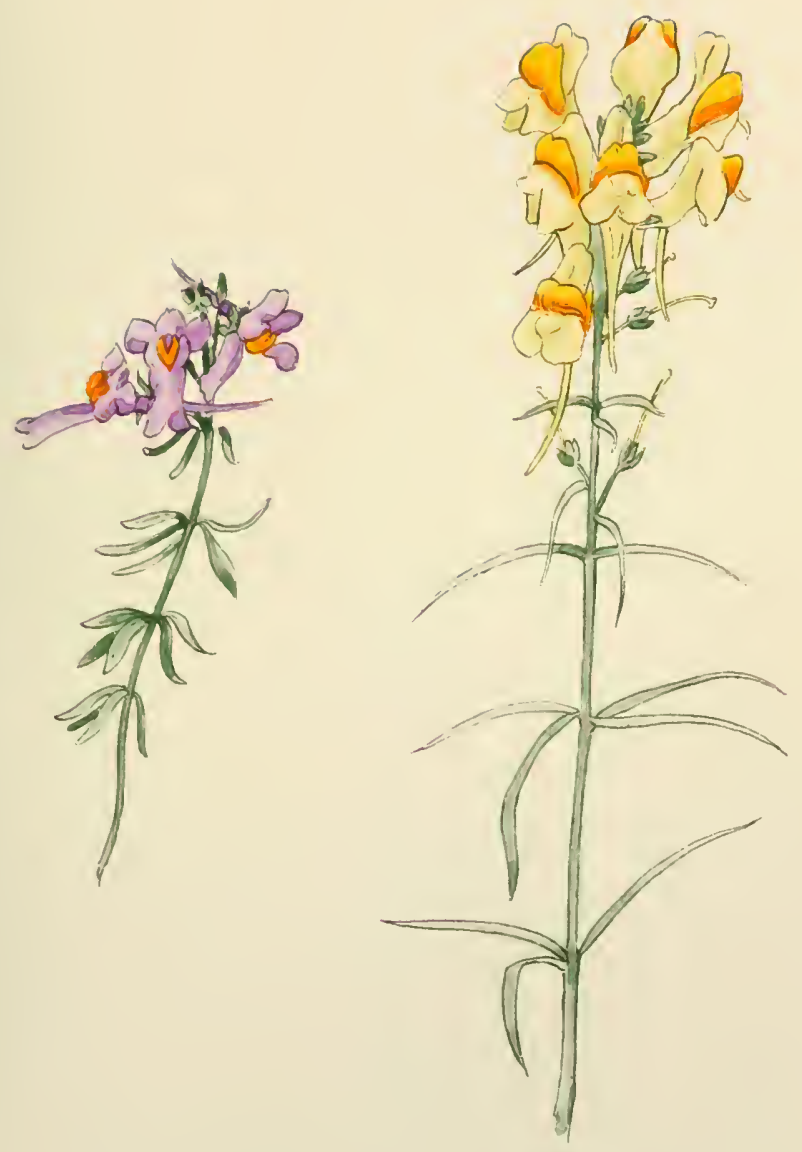

Linaria alpina.

Linaria vulgaris. 

CLXVII.
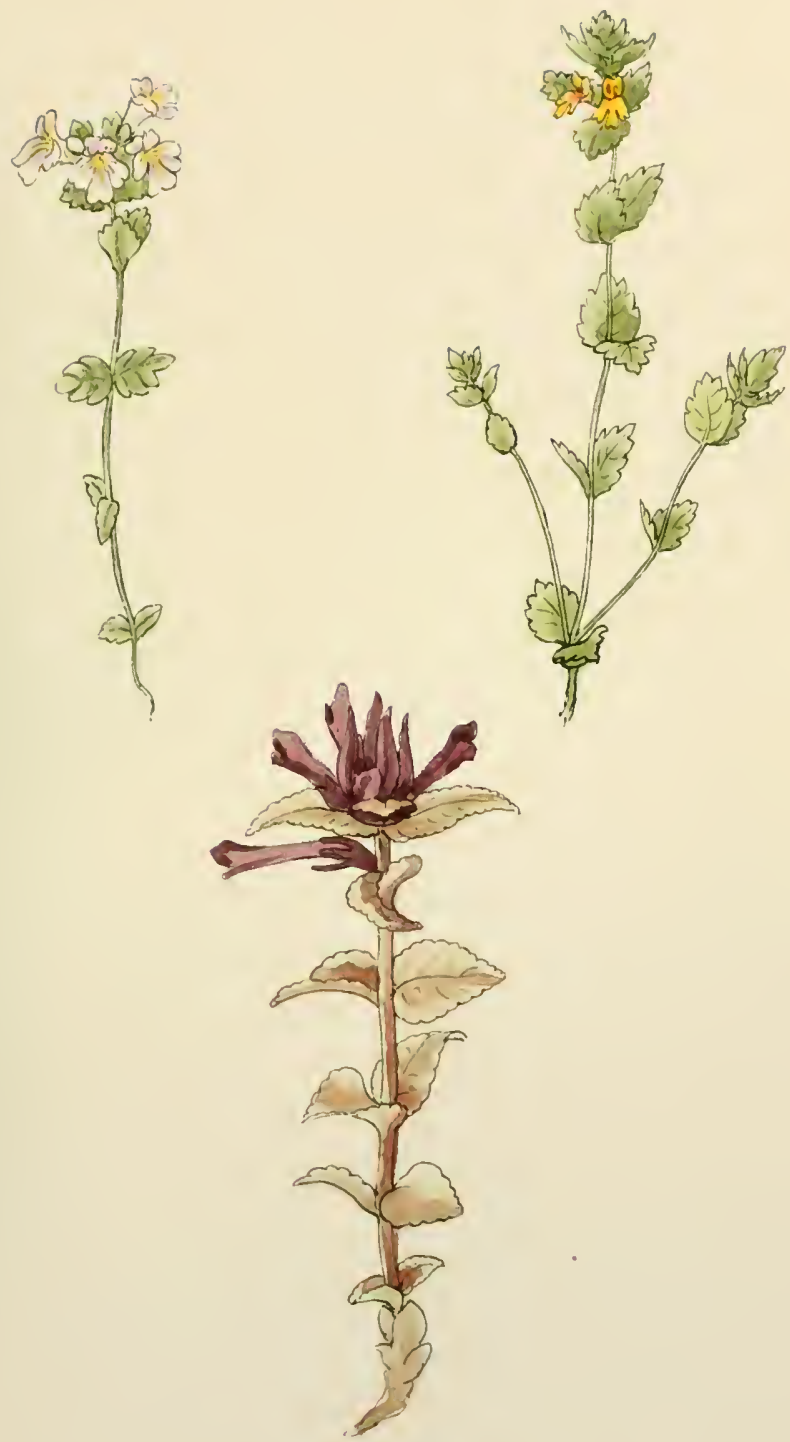

Euphrasia officinalis.

Euphrasia minima.

Bartsia alpina. 



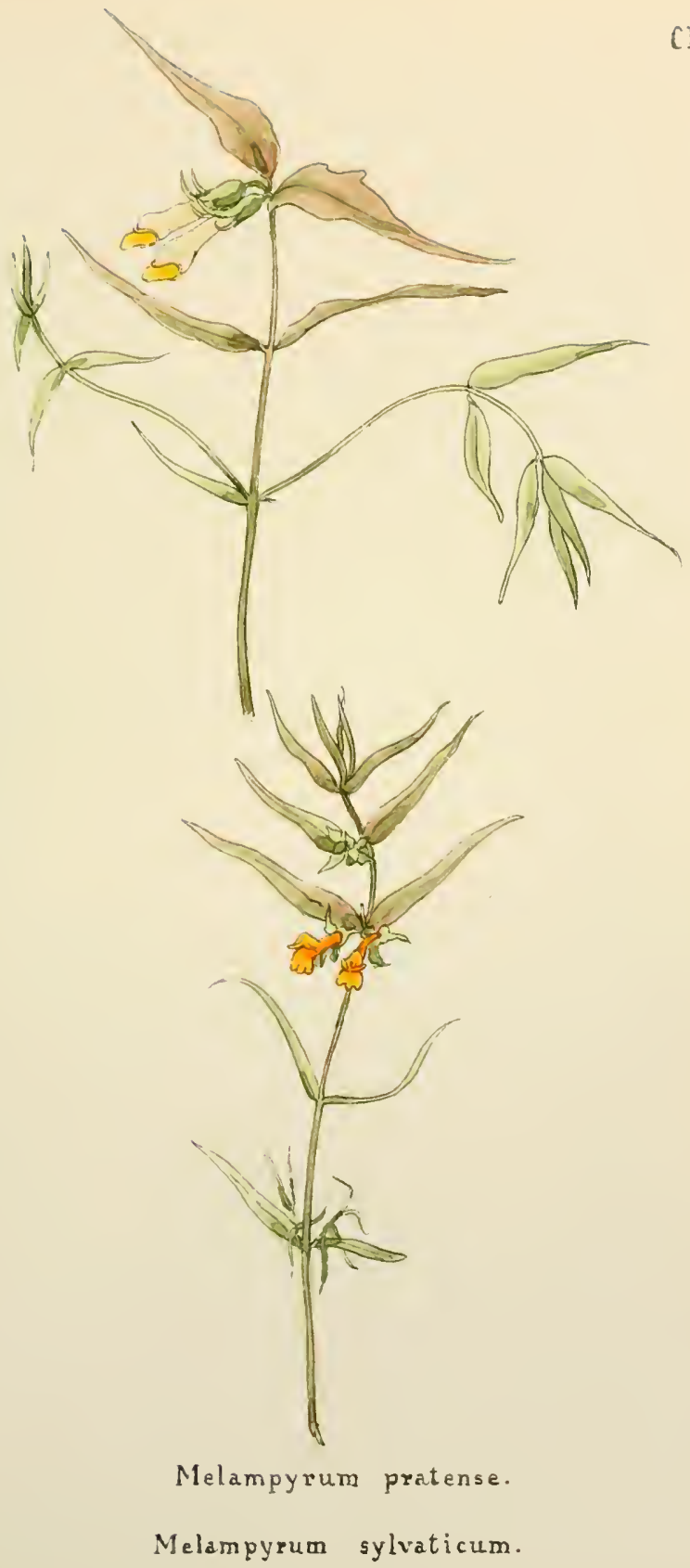

CLXVIII. 

CLXIX.
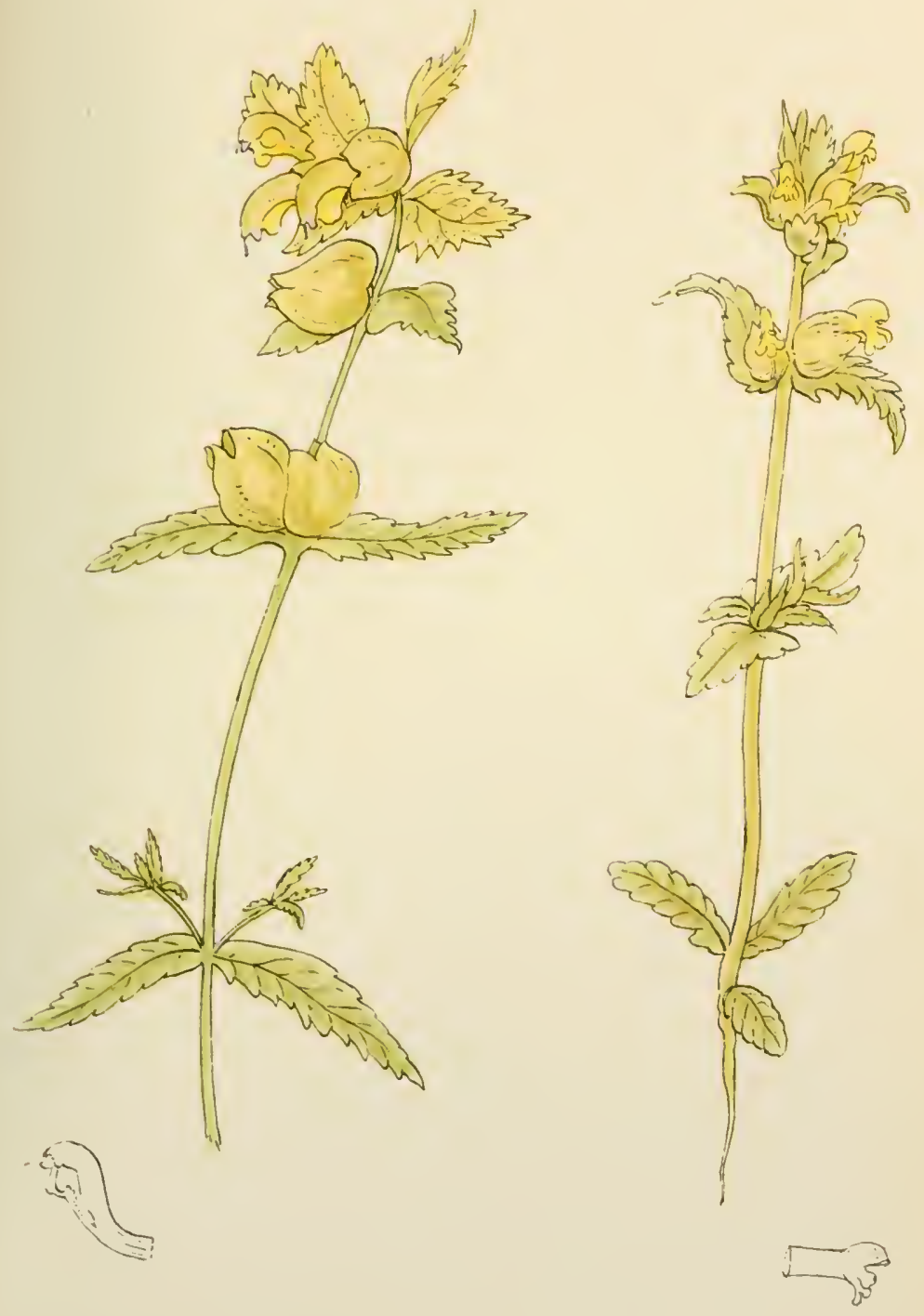

Rhinanthus major.

Rhinanthus minur. 
$\operatorname{cl} x X$

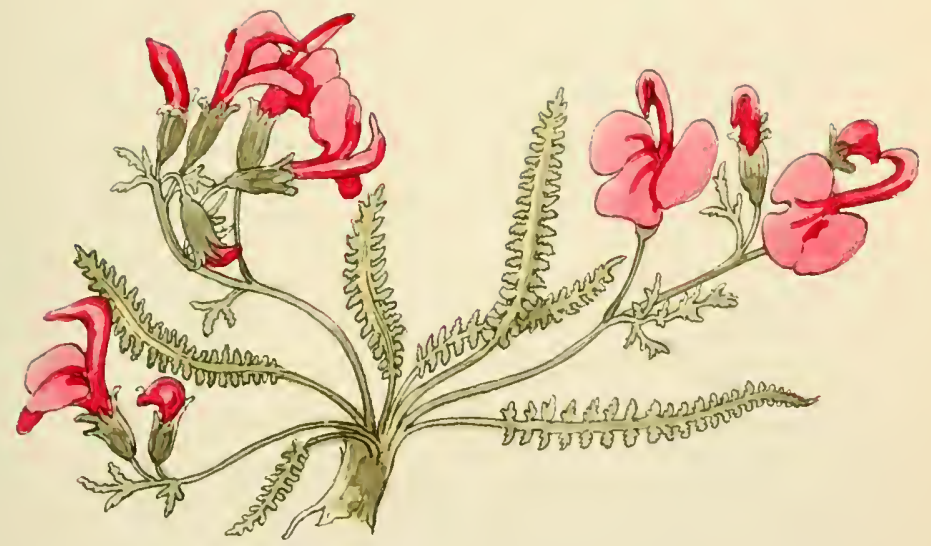

Pedicularis rostrata. 

CLXXI.
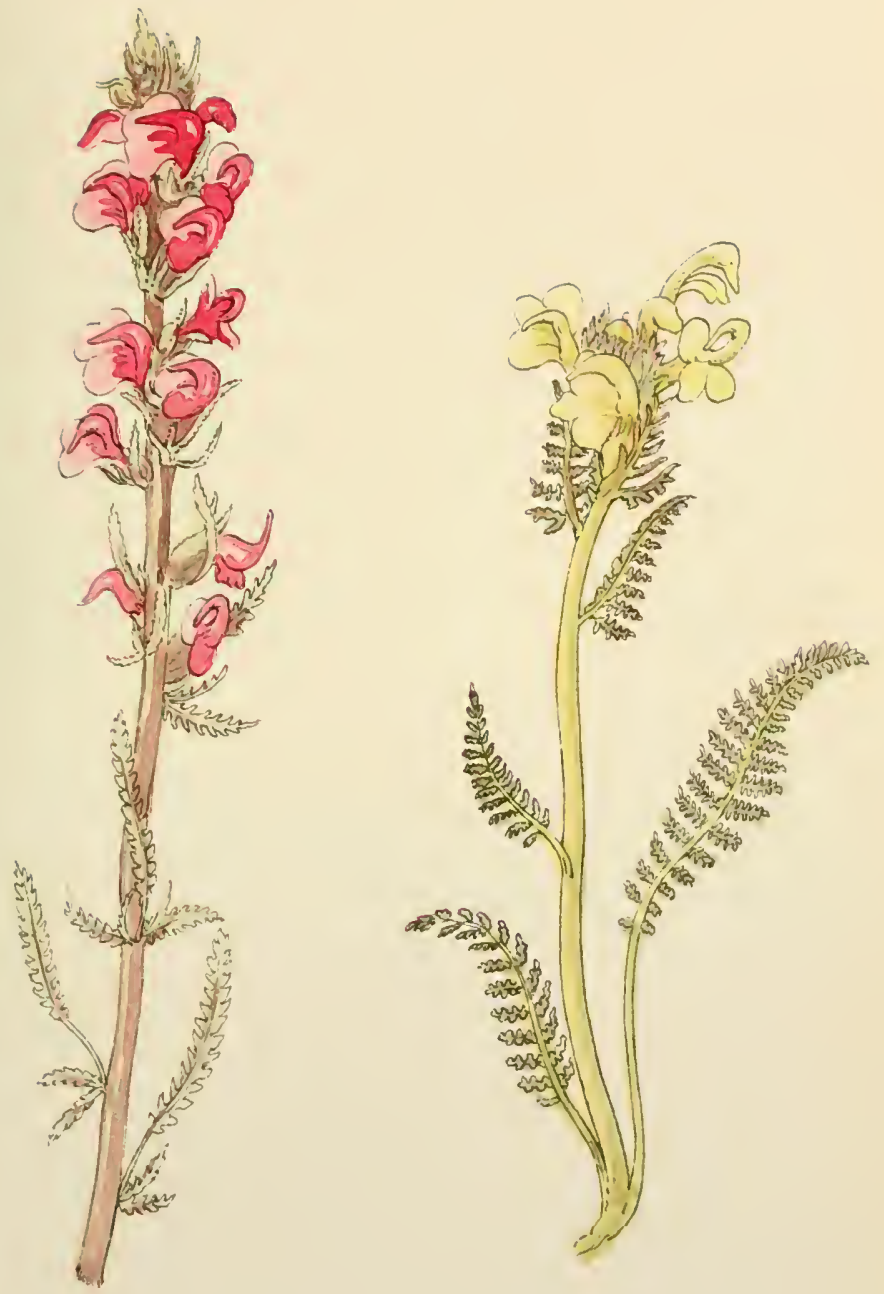

Pedicularis incarnata.

Pedicularis tuberosa. 

CLXXII.

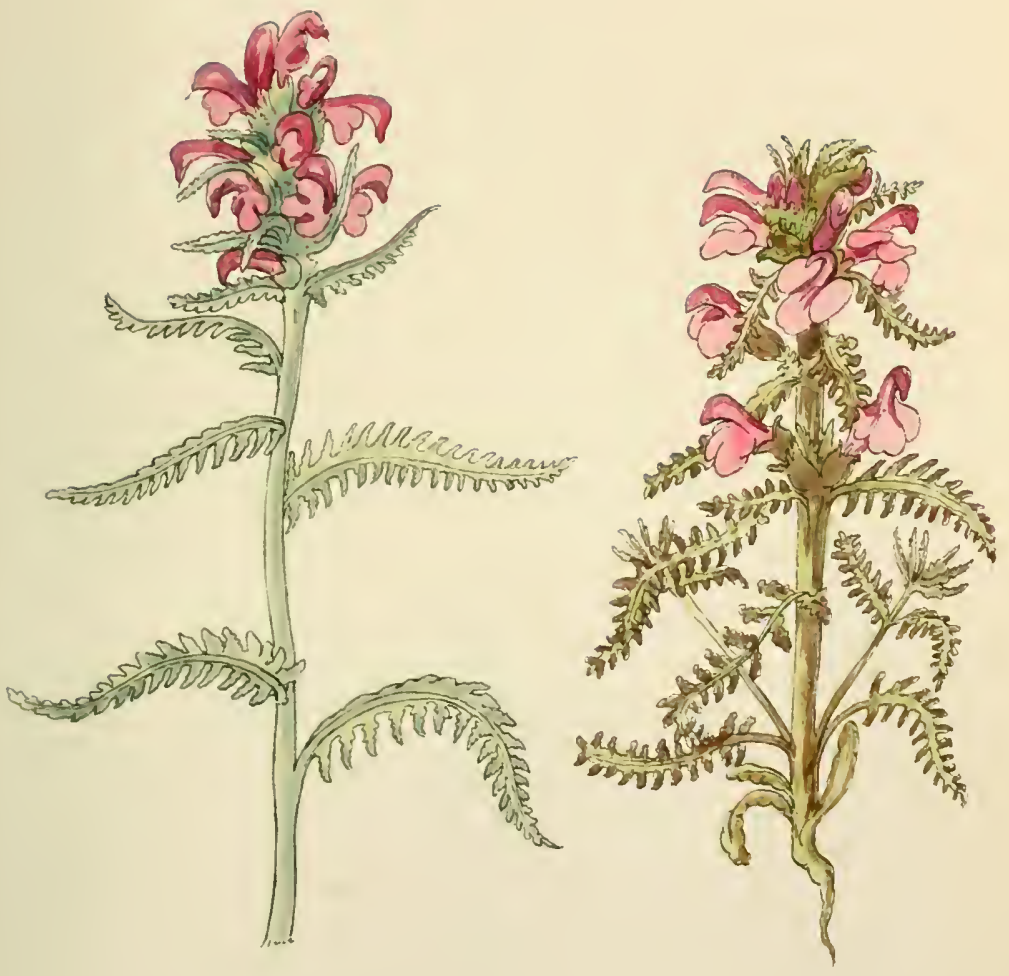

Pedicularis atrorubens. Pedicularis palustris 
CLXXIII.

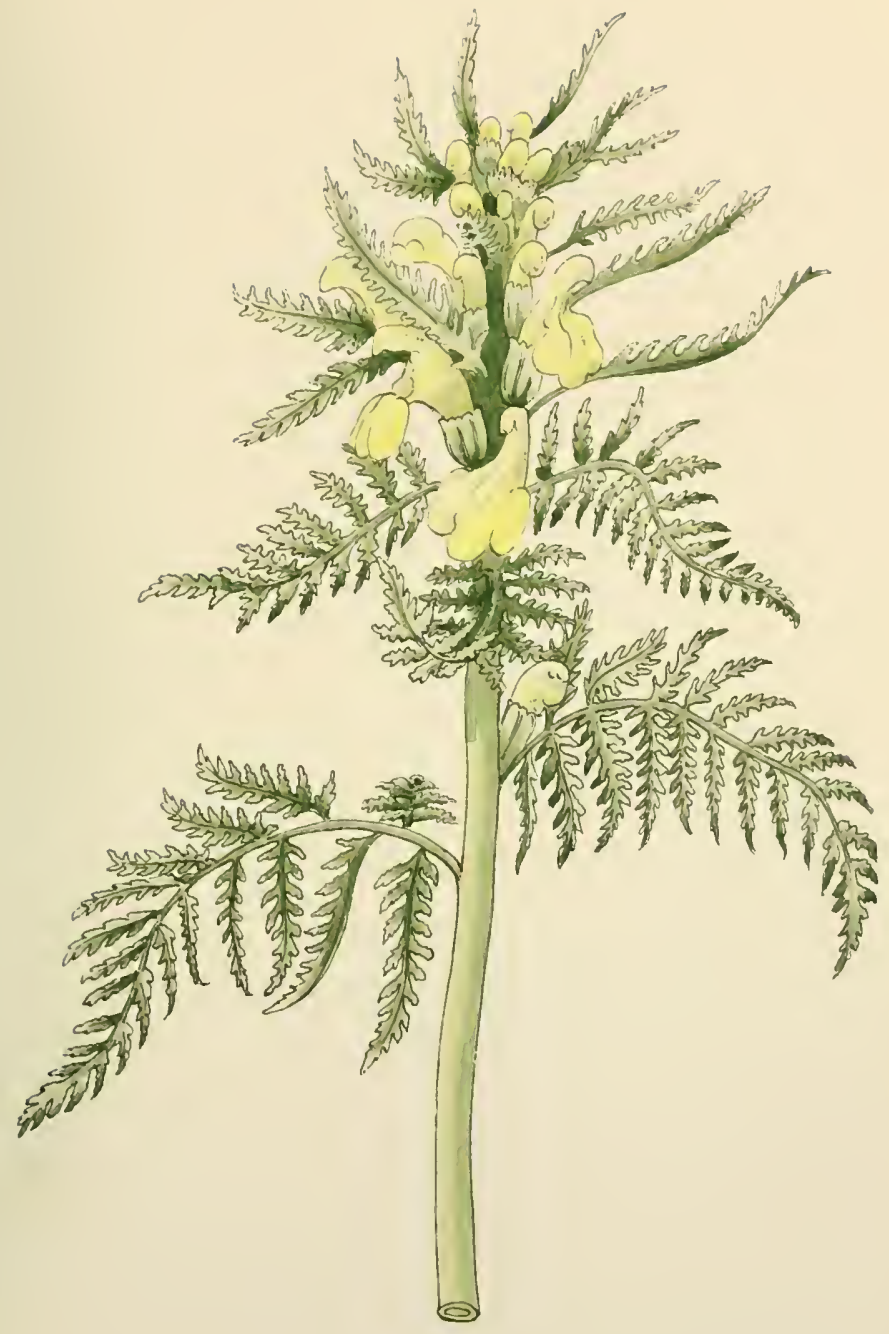

Pedicularis foliosa. 



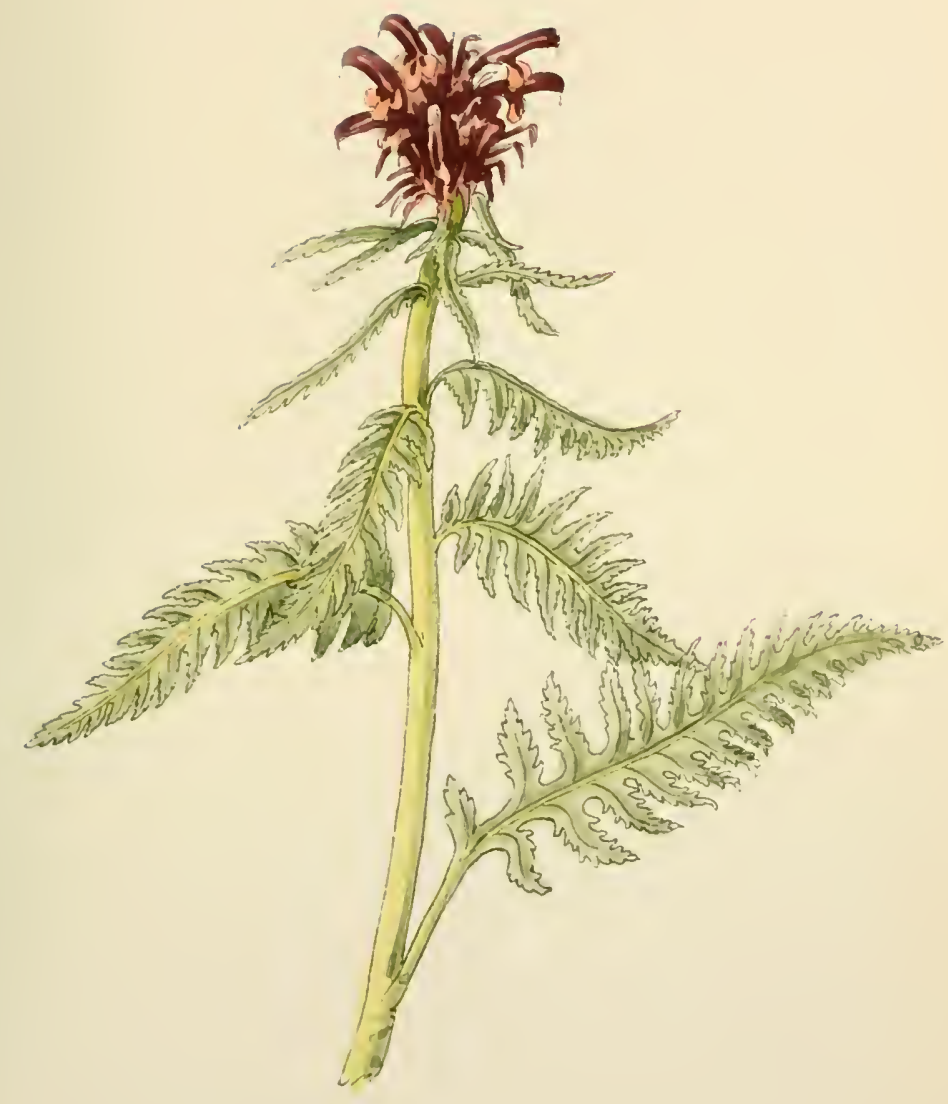

Pedicularis recutita. 

CLXXV.

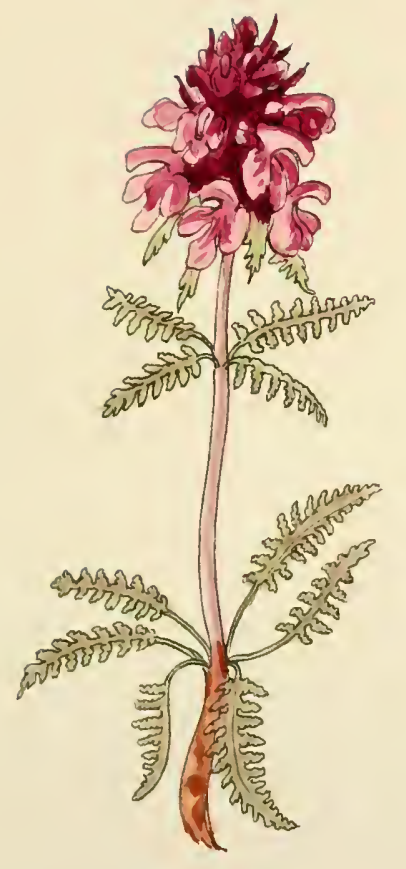

Pedicularis verticillata. 

CLXXVI.
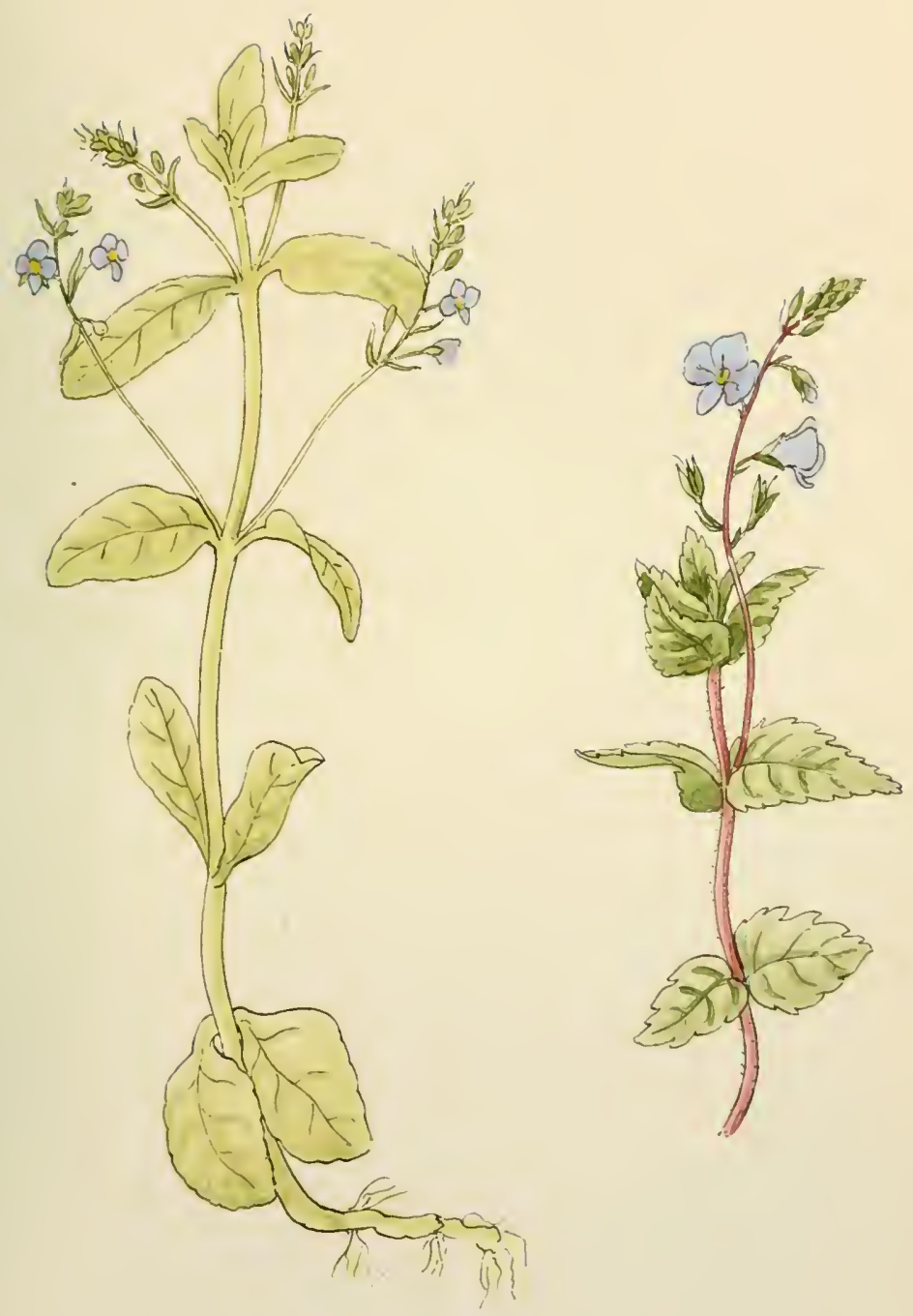

Veronica Beccabunga.

Veronica Chamadrys. 

CLXXVII.
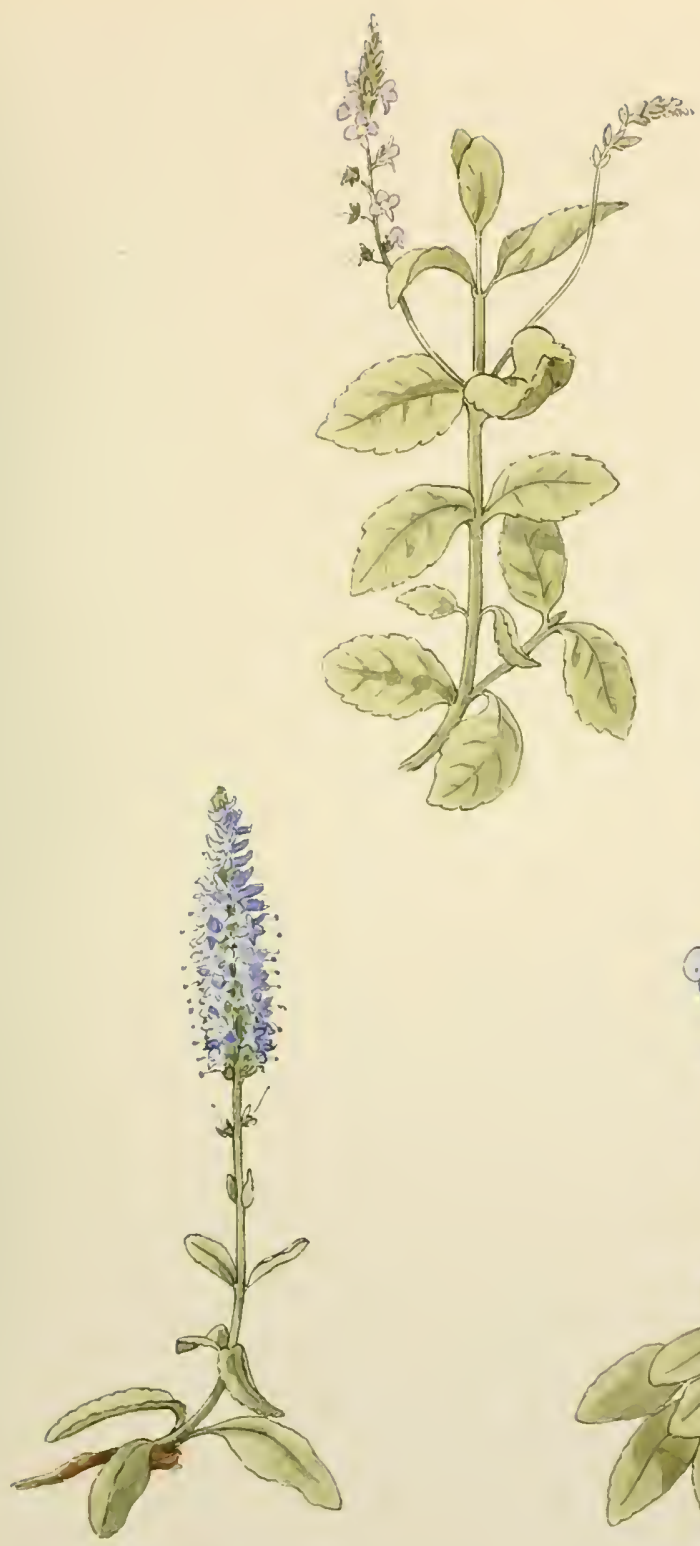

Veronica officinalis.

Veronica spicata.

Veronica bellidioïdes. 

CLXPIIII.
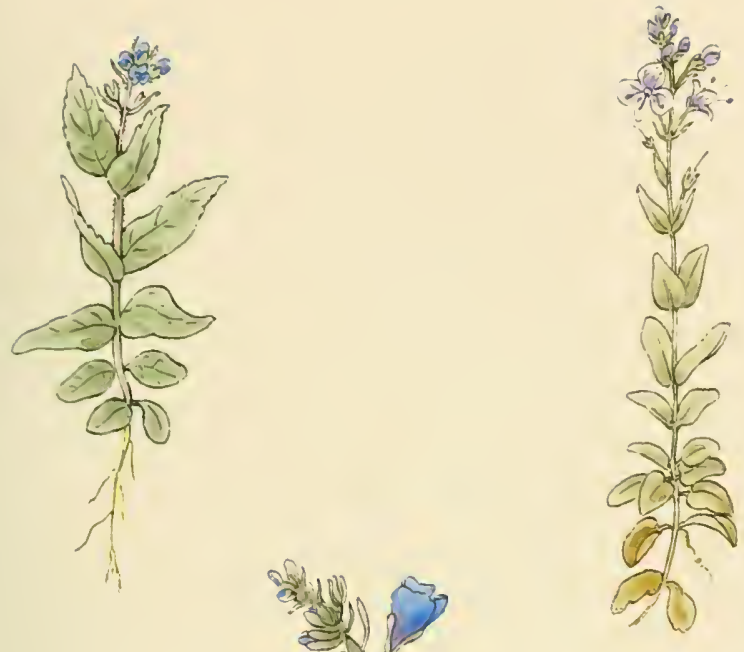

Veronica alpina.

Veronica serpyllifolia.

Veronica saxatilis. 


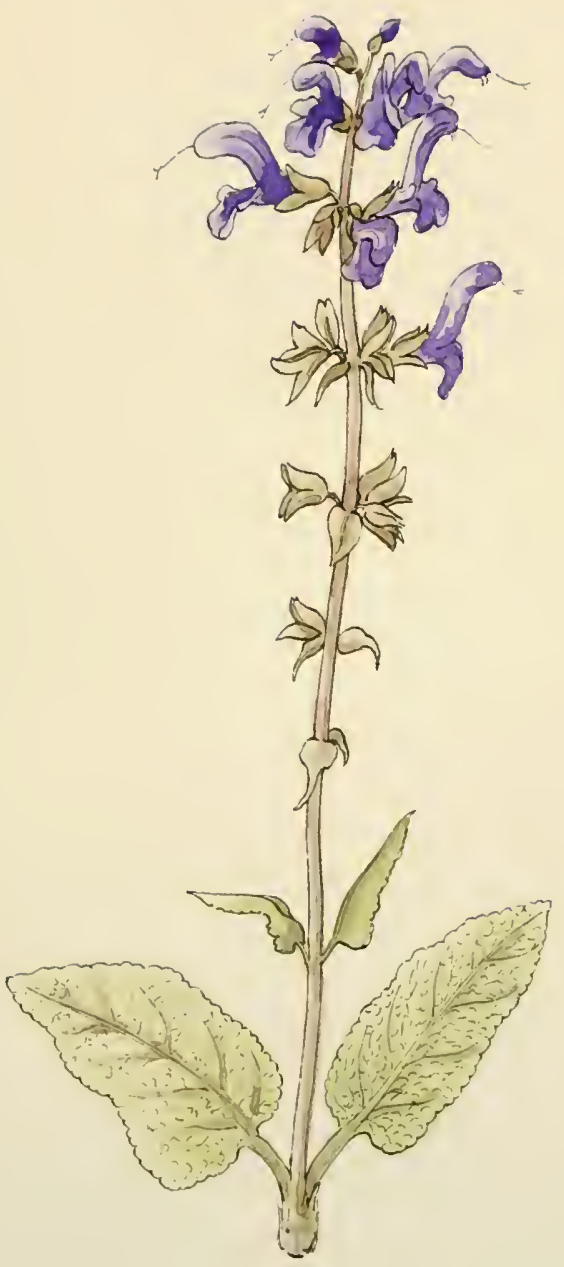

salvia pratensis. 

C LXXX.
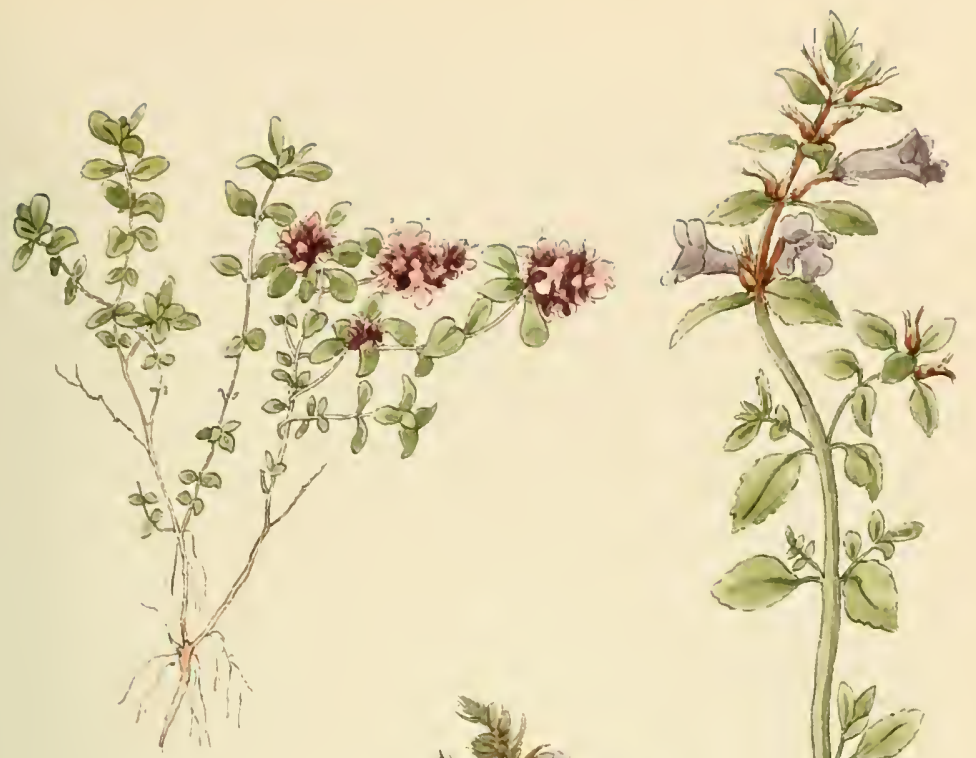

CLXXXI.

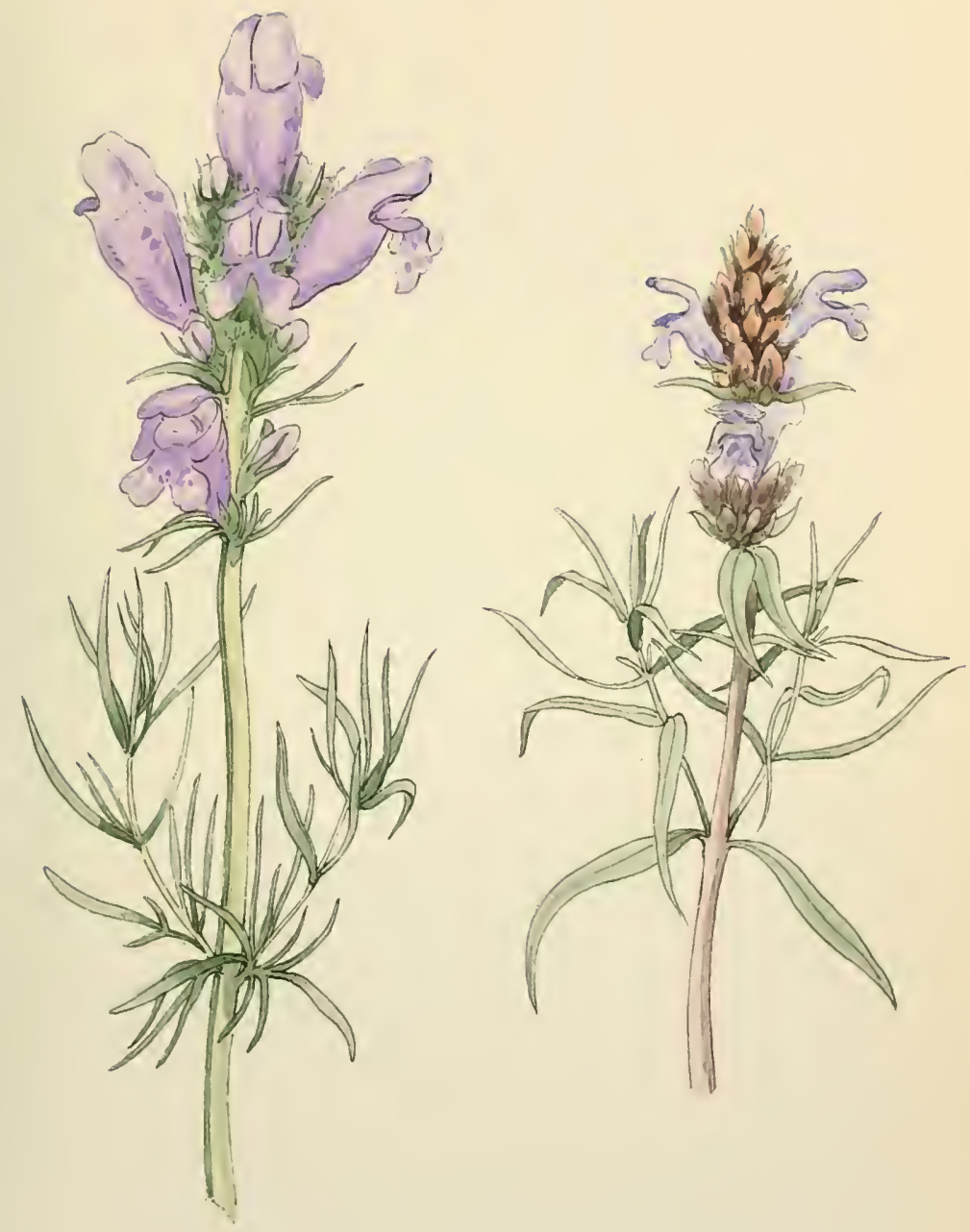

Dracocephalum austriacum. Dracocephalum Ruyschiana. 

CLXXX11.

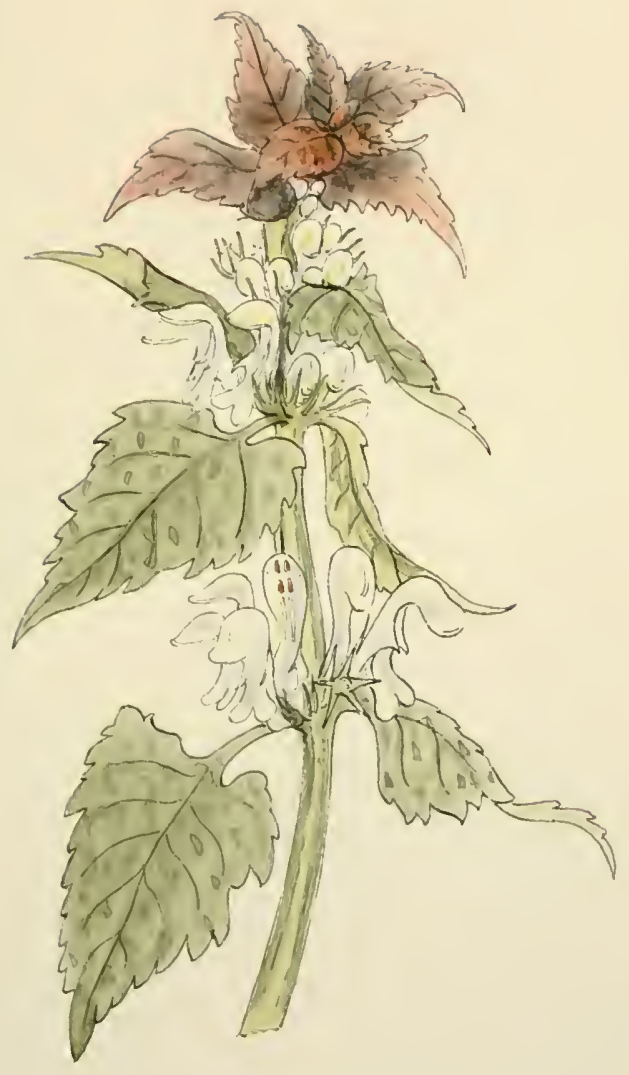

Lamium album. 



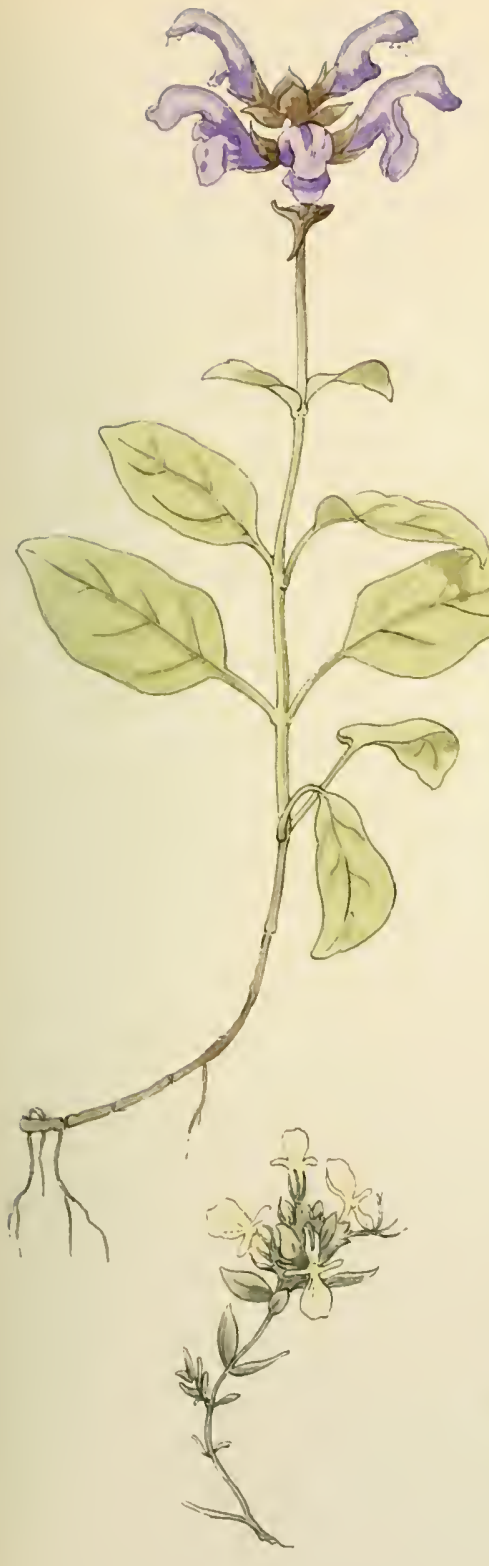

Prunella alpina.

Teucrium montanum.

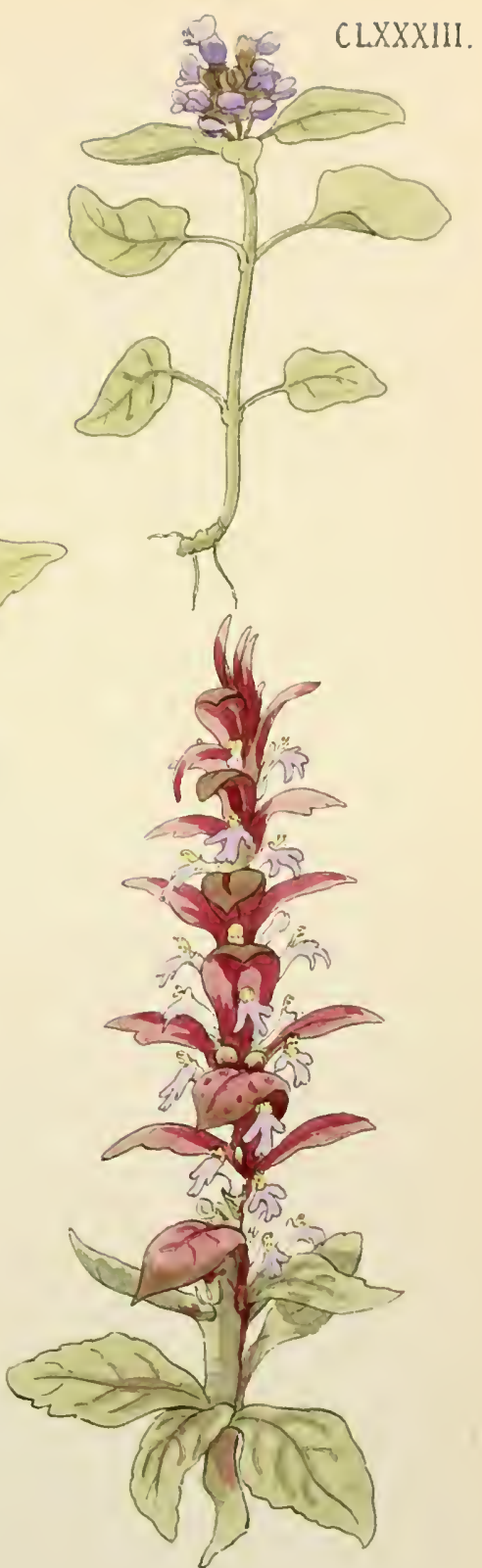

Prunella vulgaris.

Ajuga pyramidalis. 

CLXXXIV.

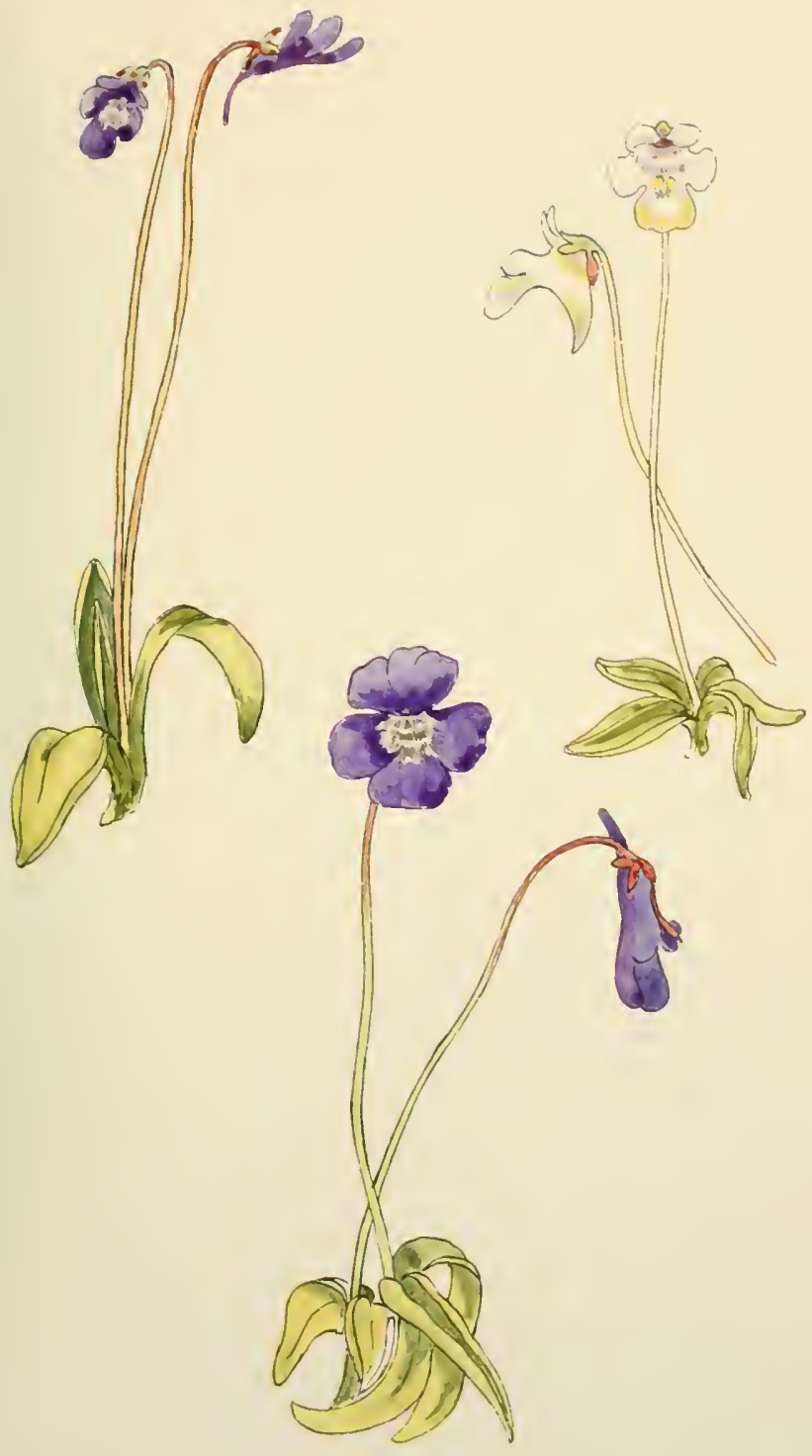

Pinguicula vulgaris.

Pinguicula alpina.

Pinguicula grandiflora. 
CLXXXV.

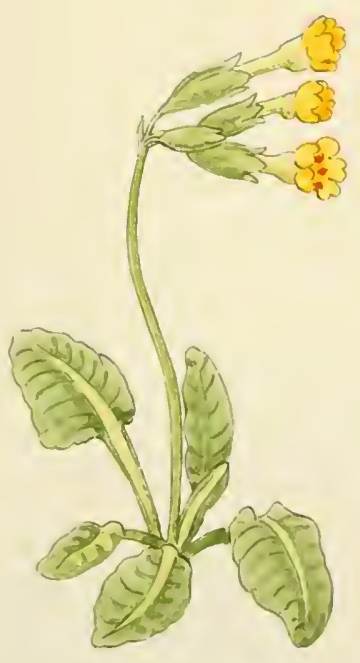

Primula officinalis. 


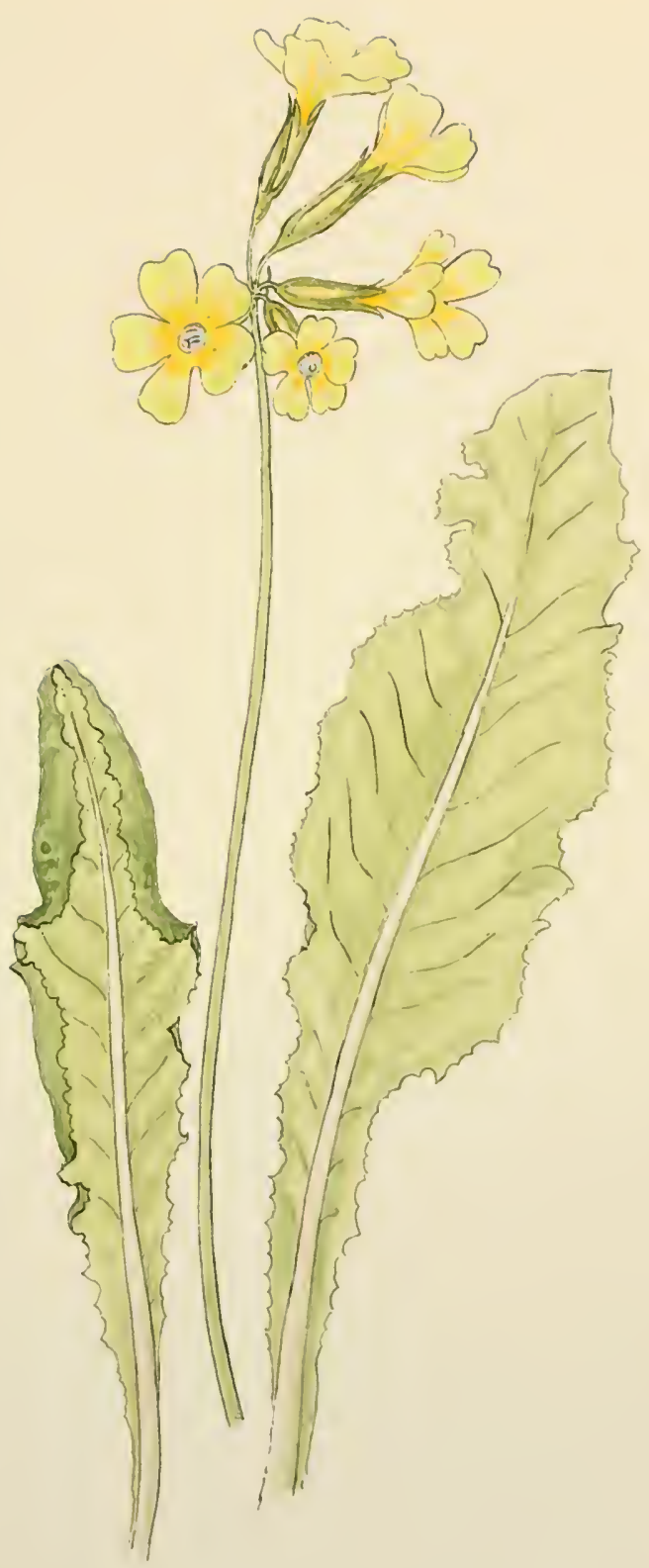

CLXXXVI.

Primula elatior. 

CLXXXVII .

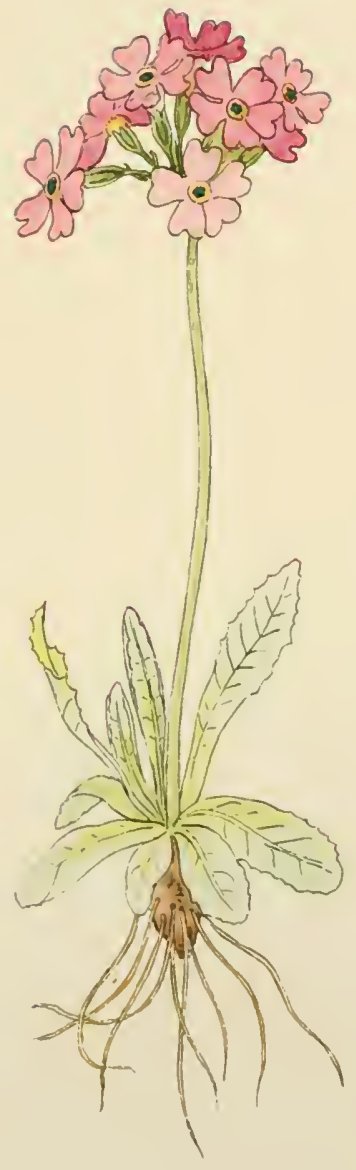

Primula farinosa. 

CLXXXVIII

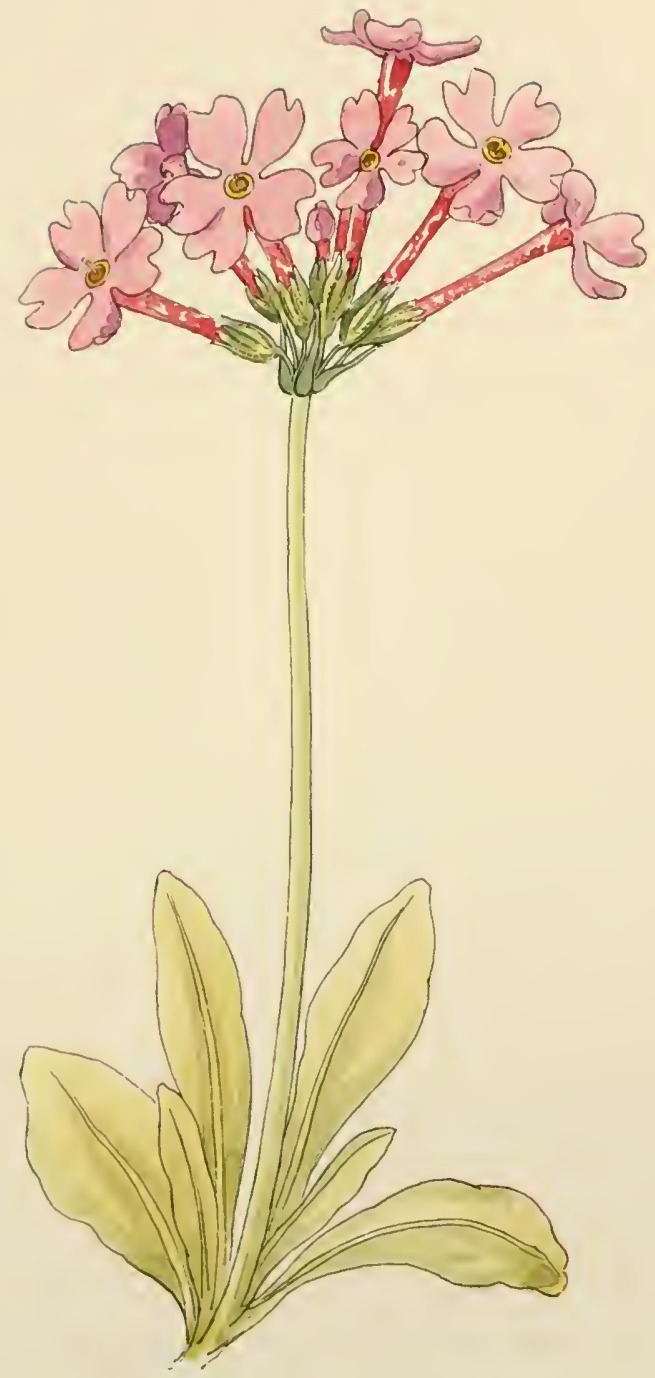

Primula Jongiflora. 

CLXXXIX .

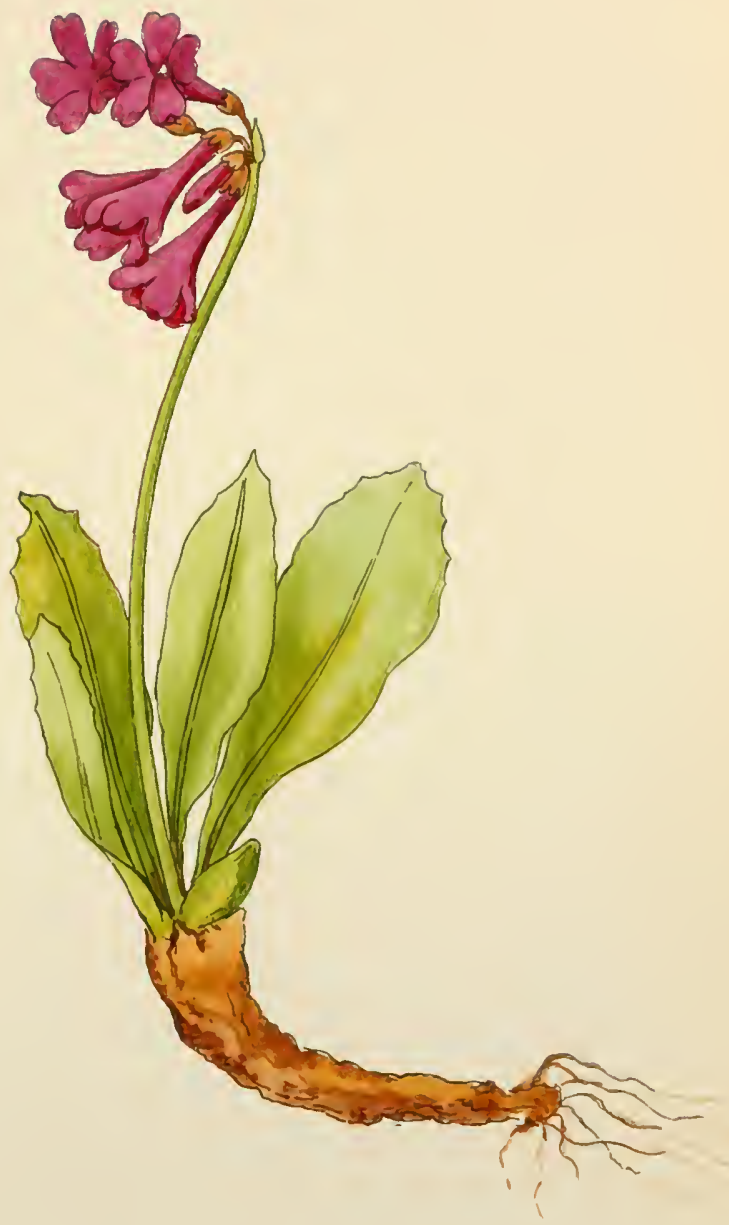

Primula graveolens. 



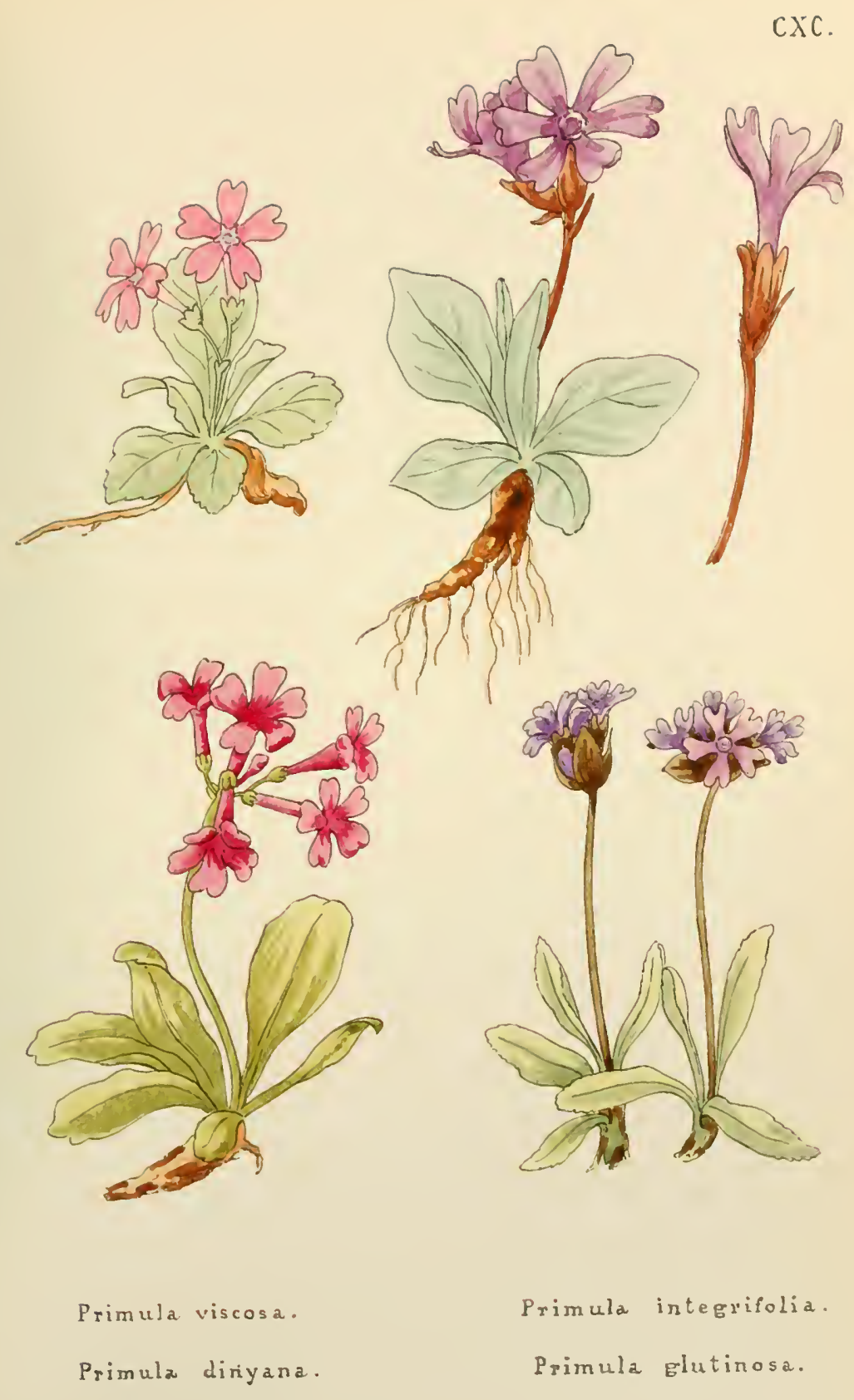



CXCI.
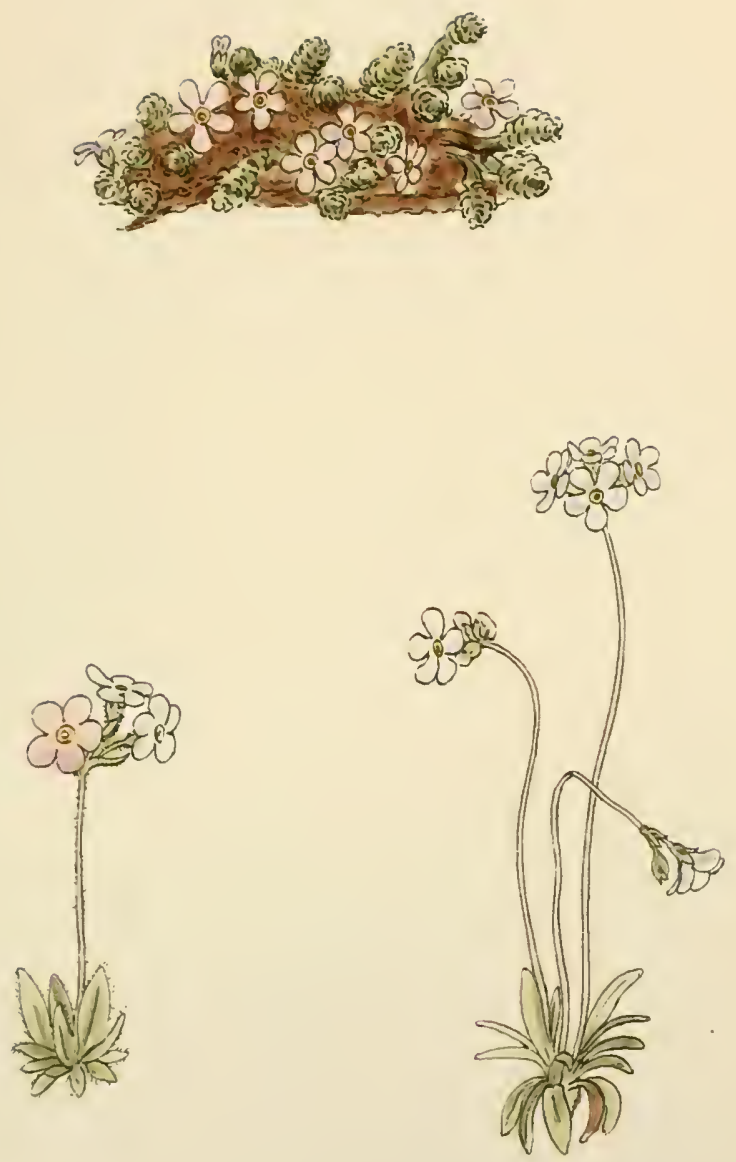

Androsace glacialis.

Andrusace Chamæjasme. Androsace obtusifolia. 

CXCII.
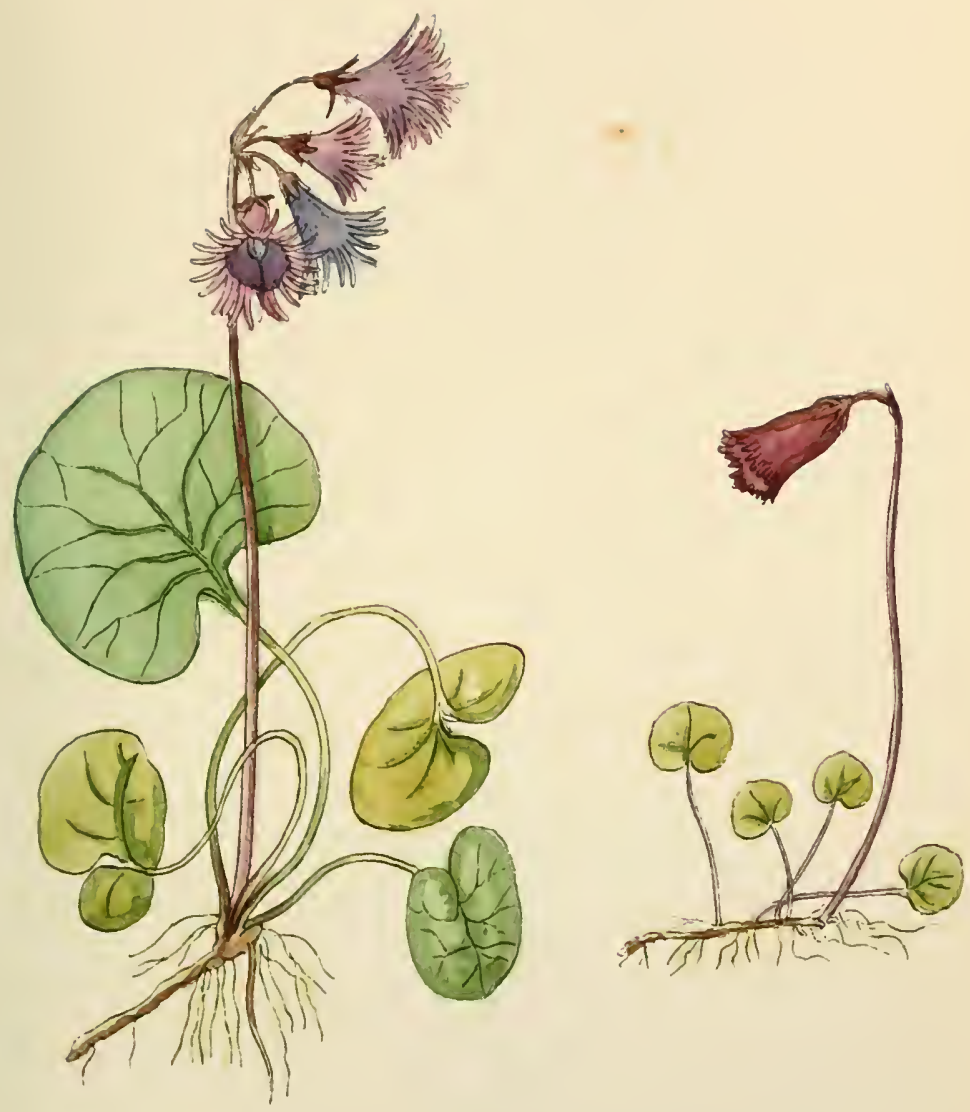

Soldanella alpina.

Soldanella pusilla. 

cXCIII.
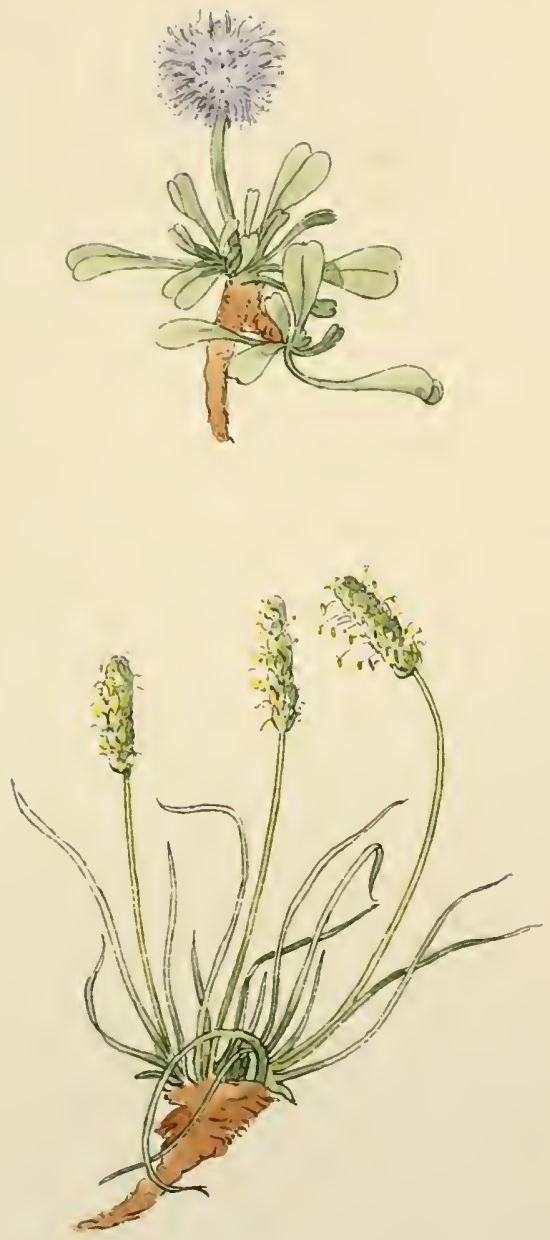

Glubularia cordifolia.

Plantago alpina . 



\section{CXCIV.}
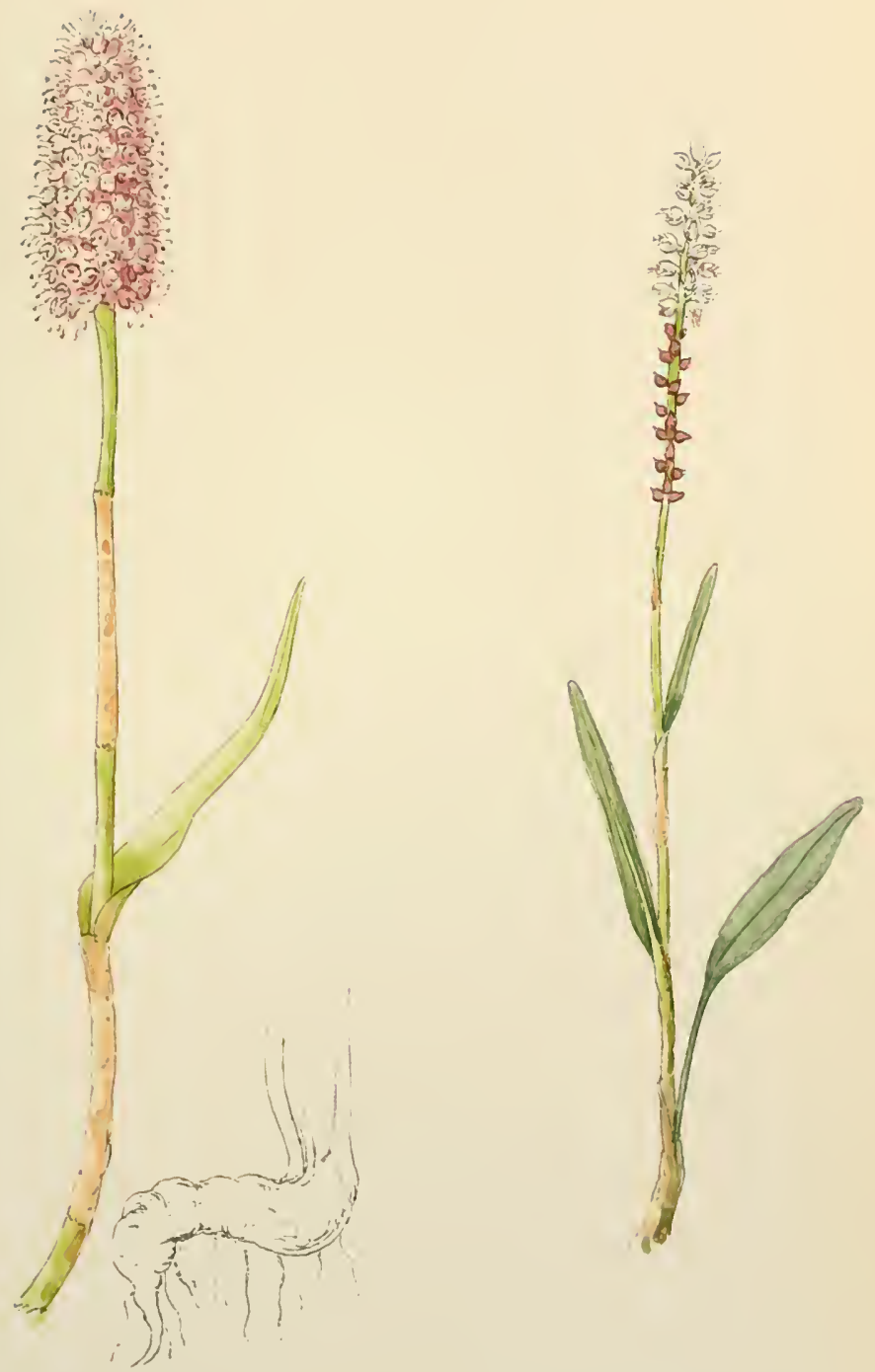

Polygonum Bistorta.

Polygonum viviparum . 

CXCV.

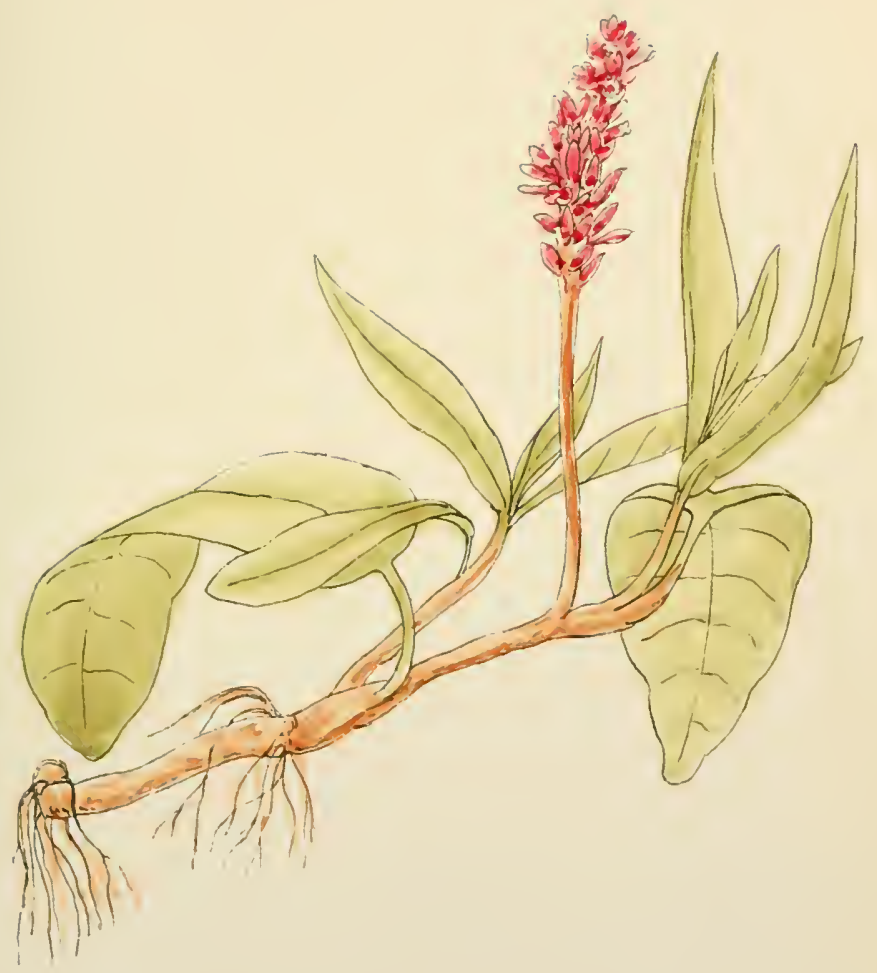

Polygonum amphibium . 

CXCVI.
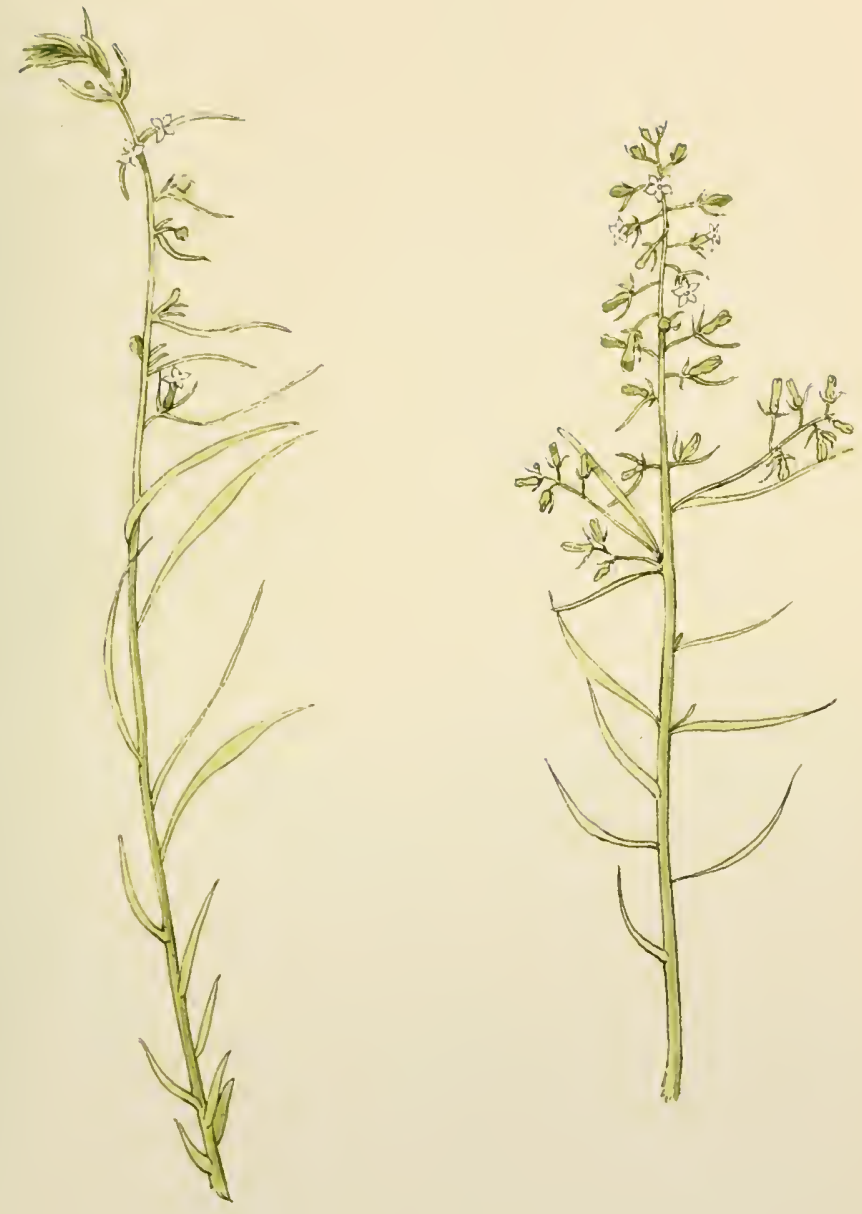

Thesium alpinum .

Thesium pratense. 

CXCVIl.

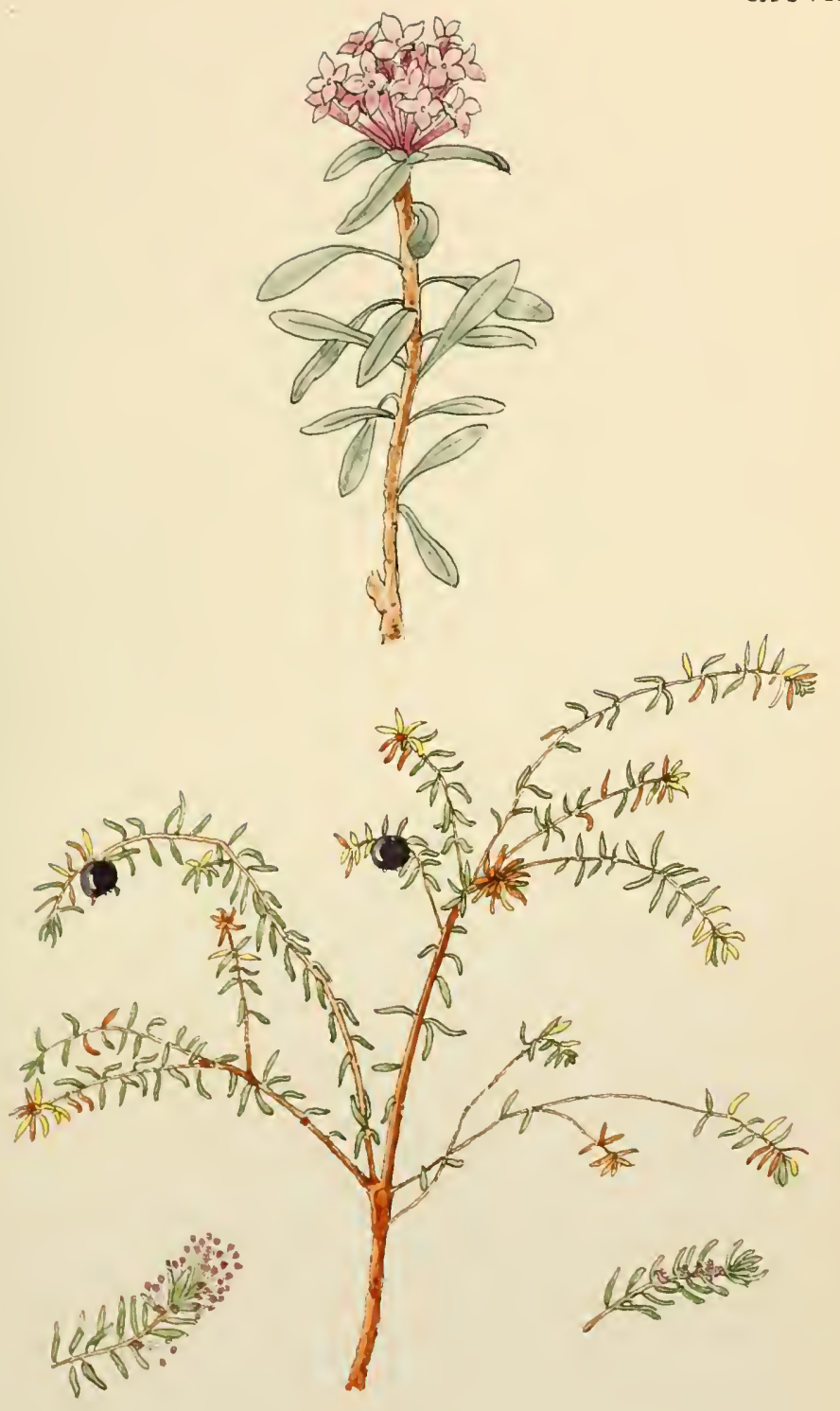

Daphne striata.

Empetrum nigrum. 



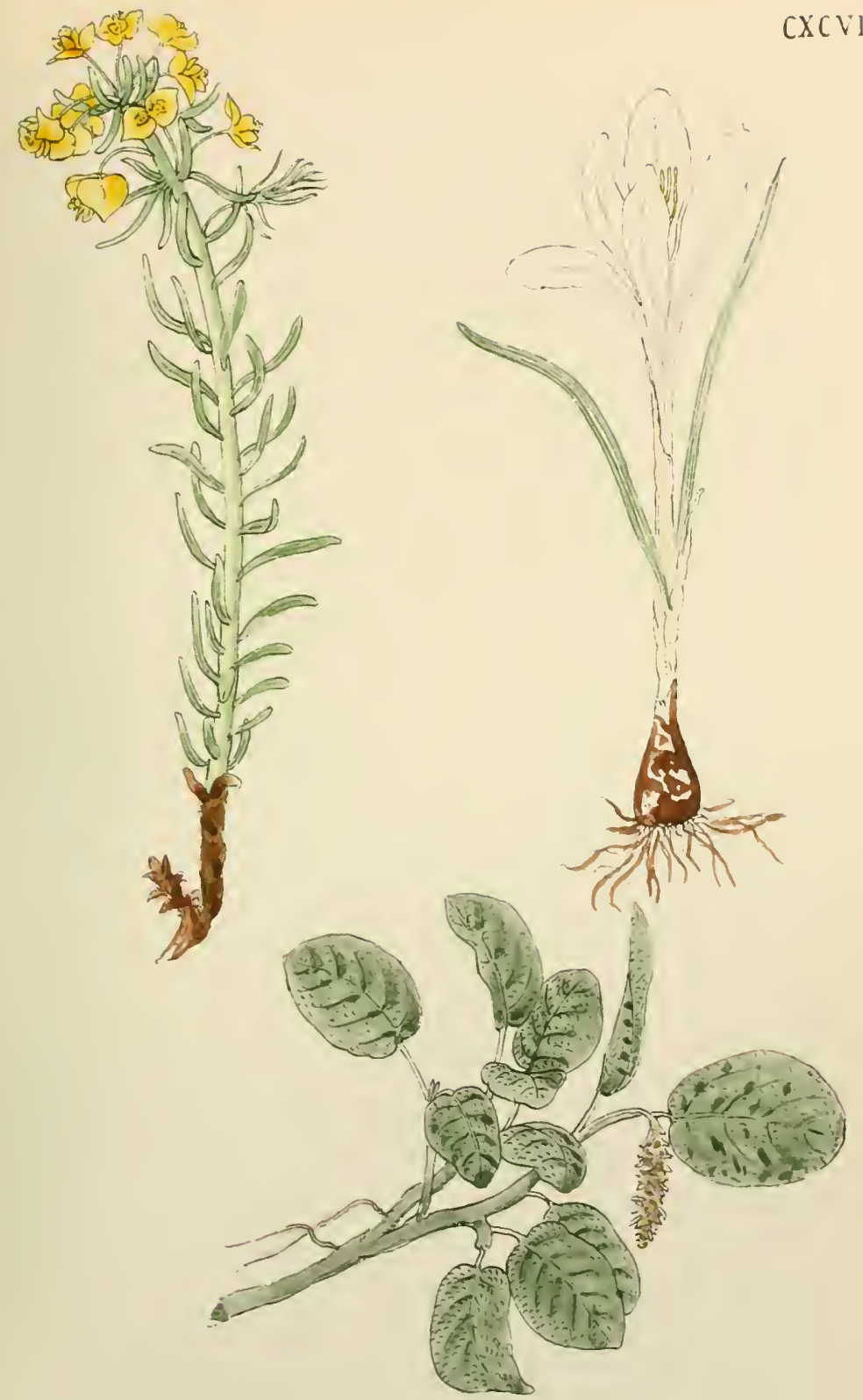

Euphorbia Cyparissias.

Crocus vernus.

Salix reticulata. 

CXCIX.
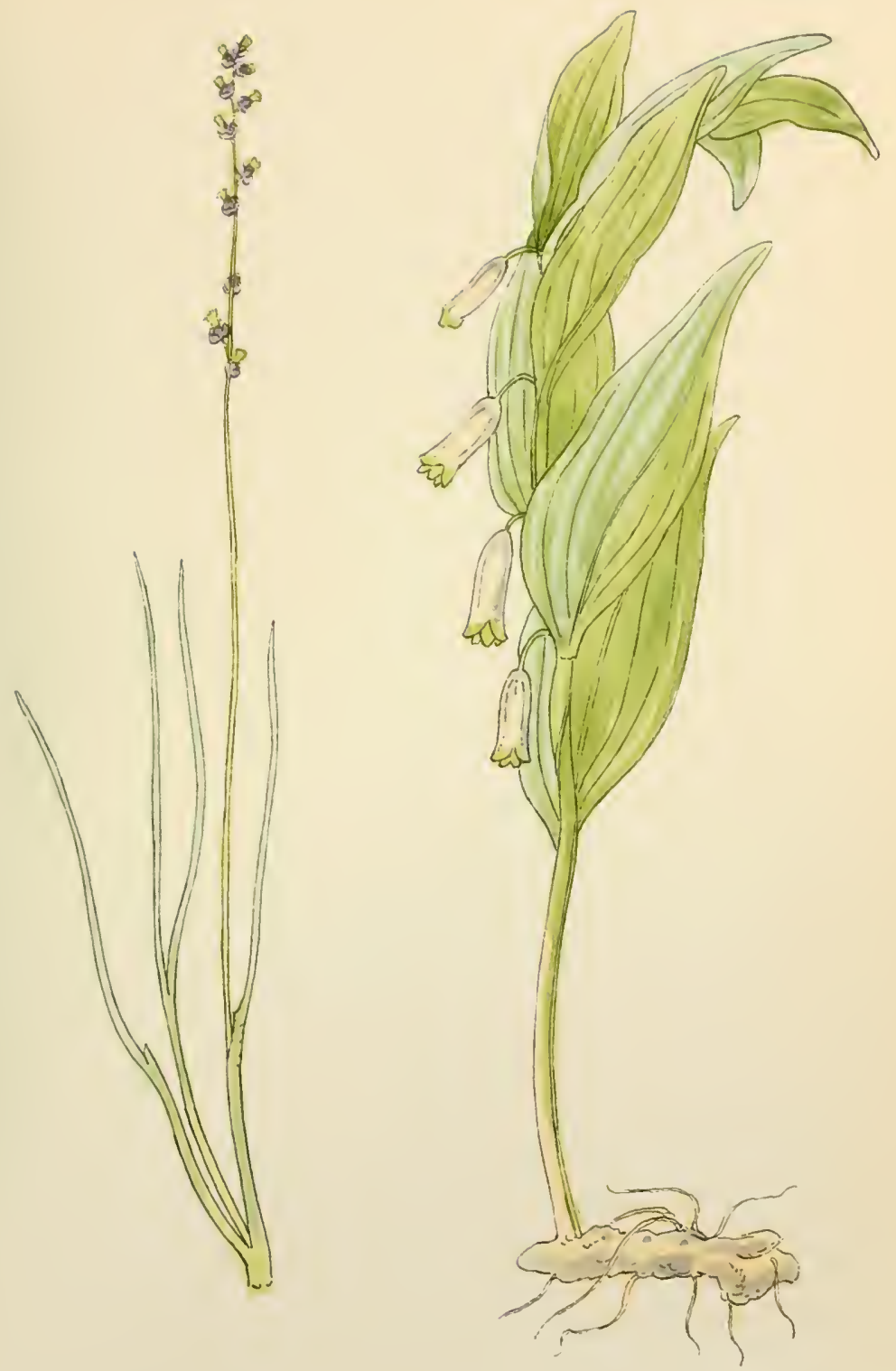

Triglochin palustre.

Polygonatum rulgare. 

c C.

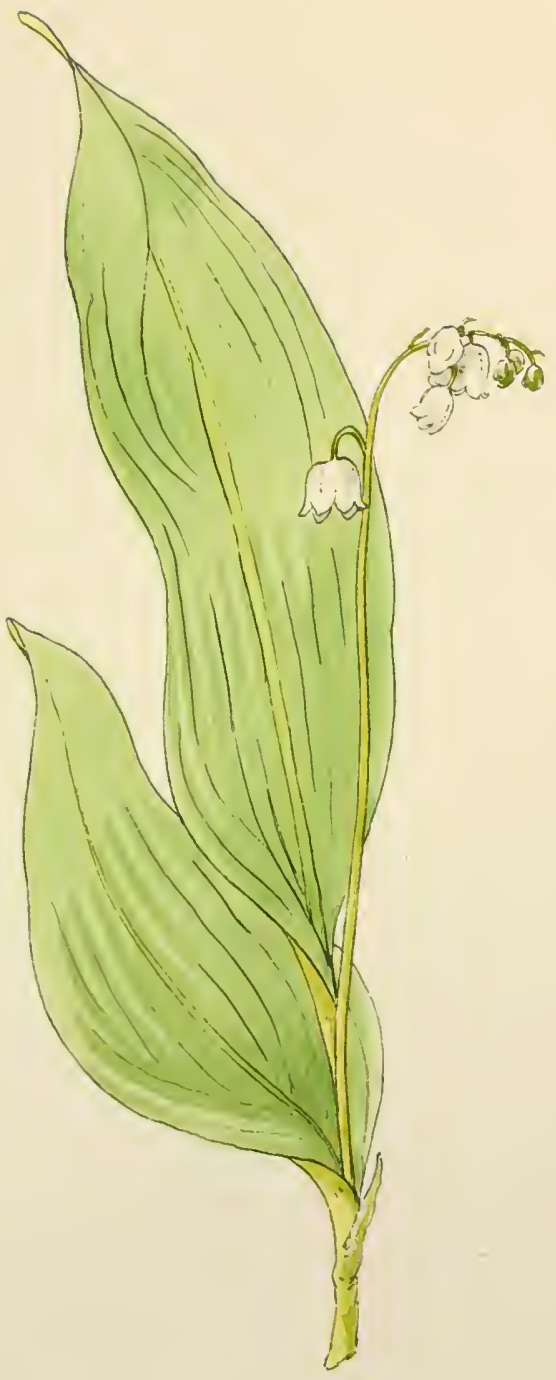

Convallaria majalis. 

CCl.

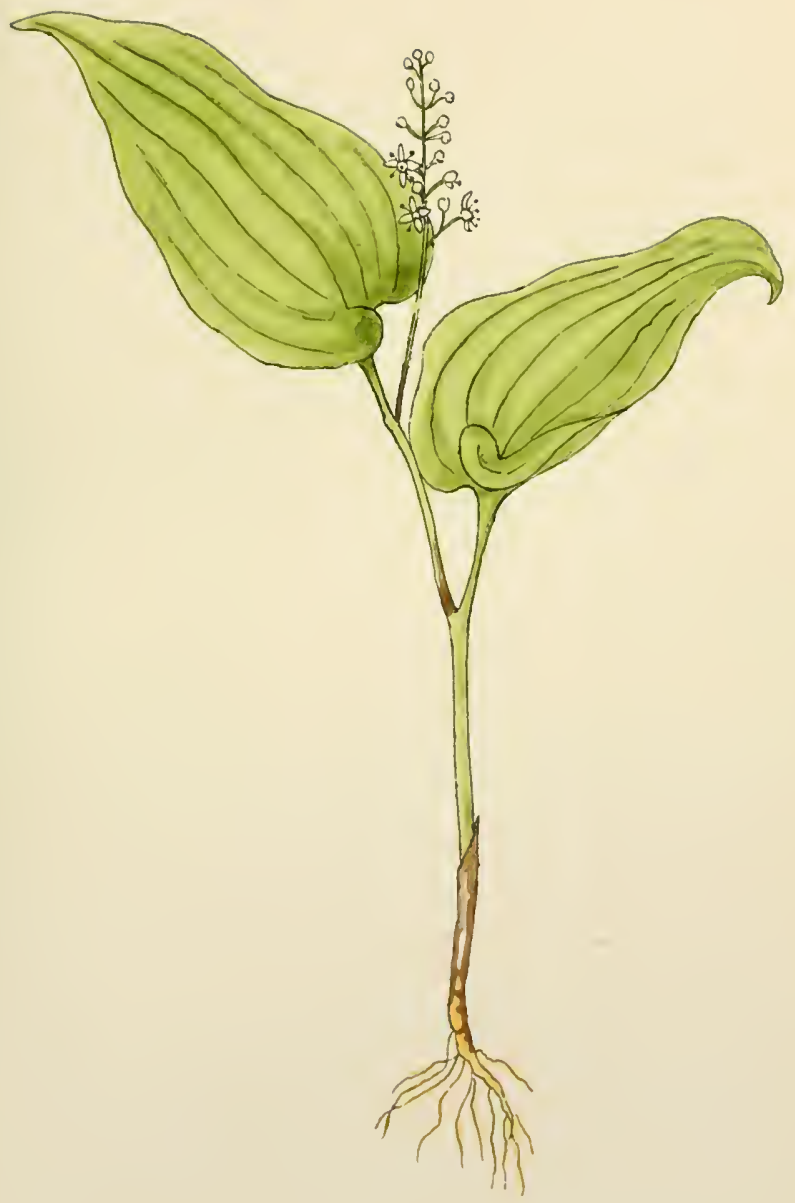

Maiarthemum bifolium. 
CCII.

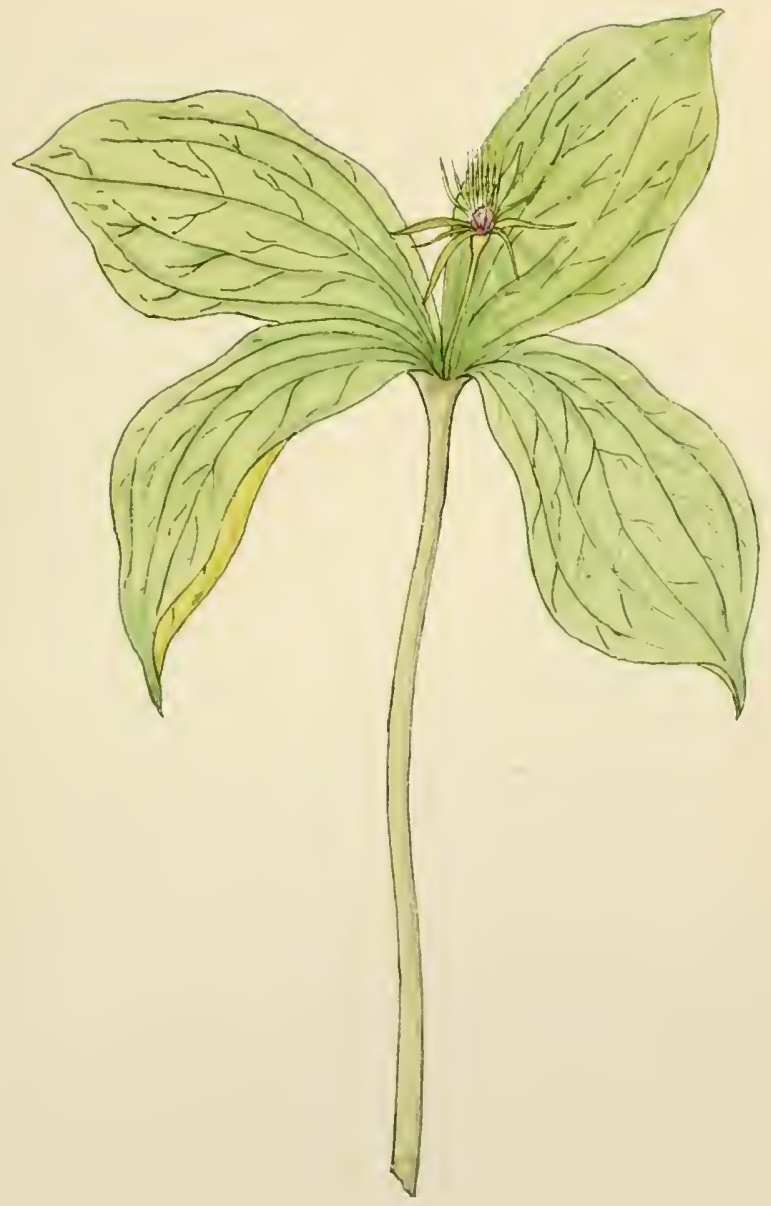

Paris quadrifolia. 
cCIII.
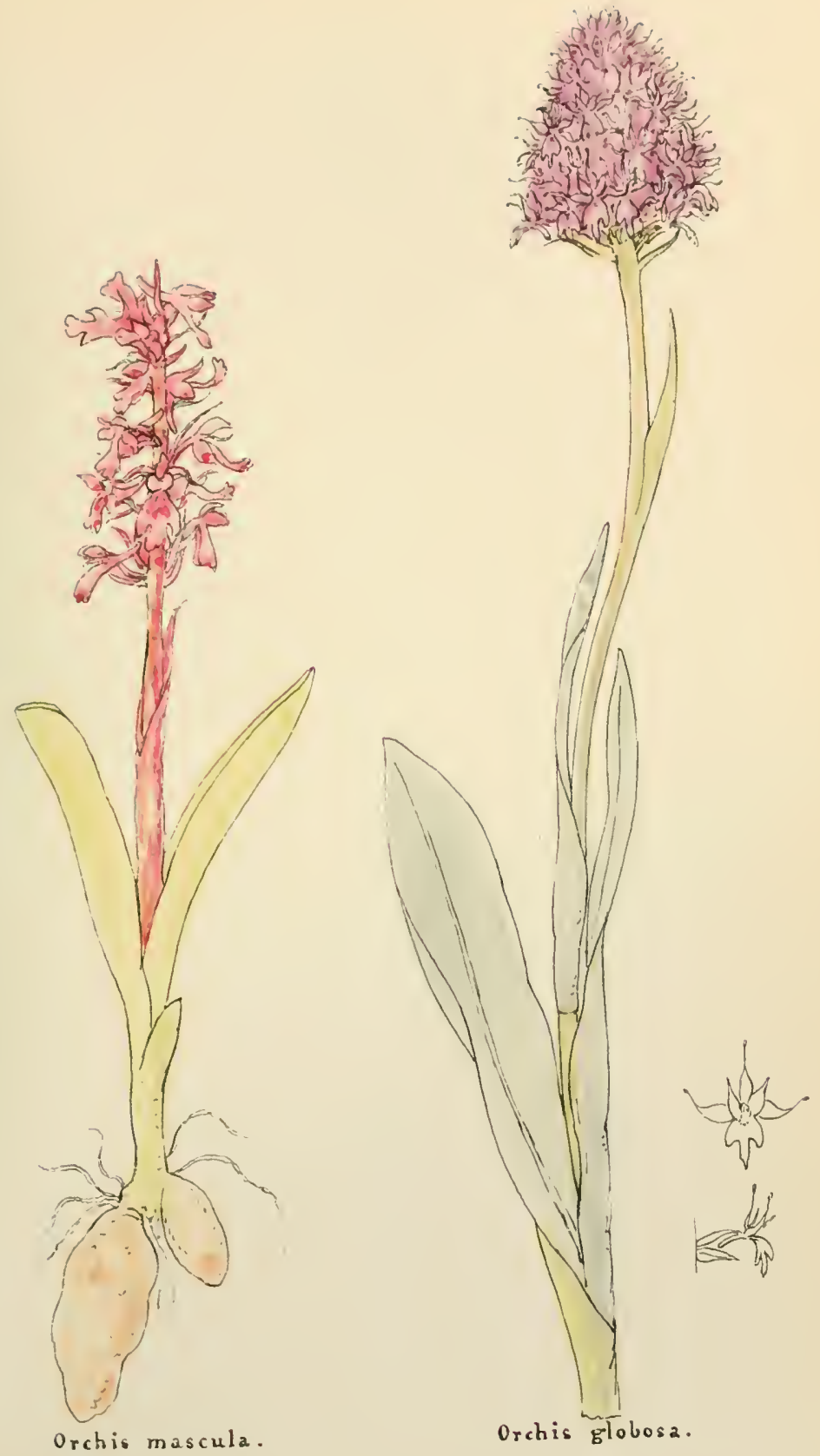



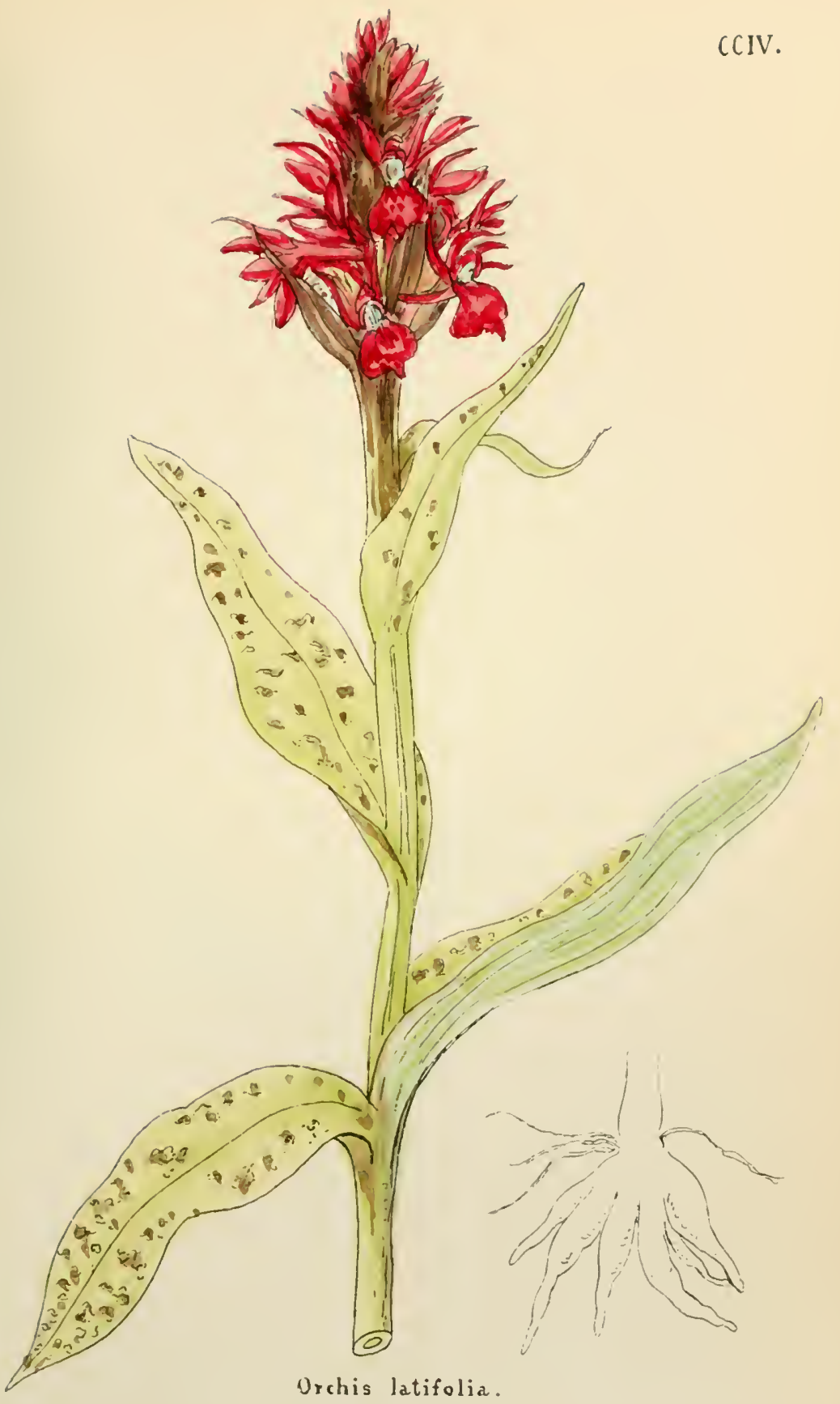



$\mathrm{CCV}$

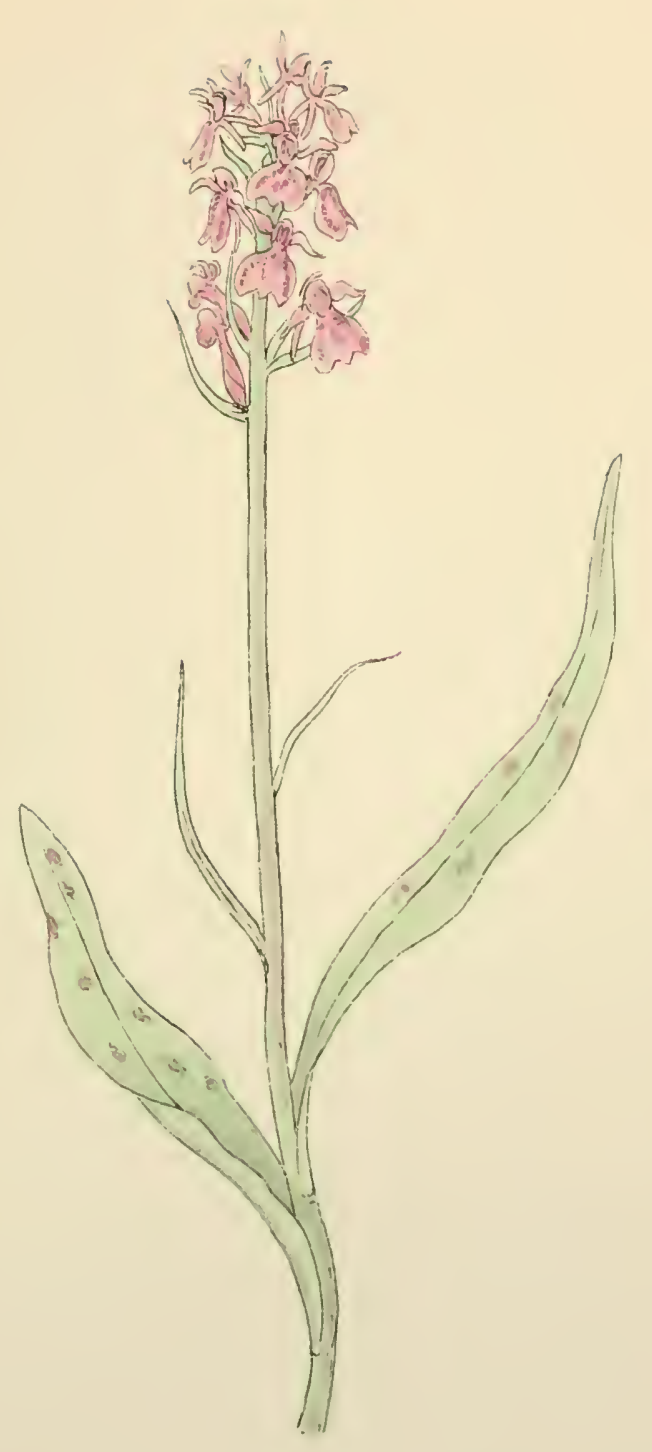



CCVI.
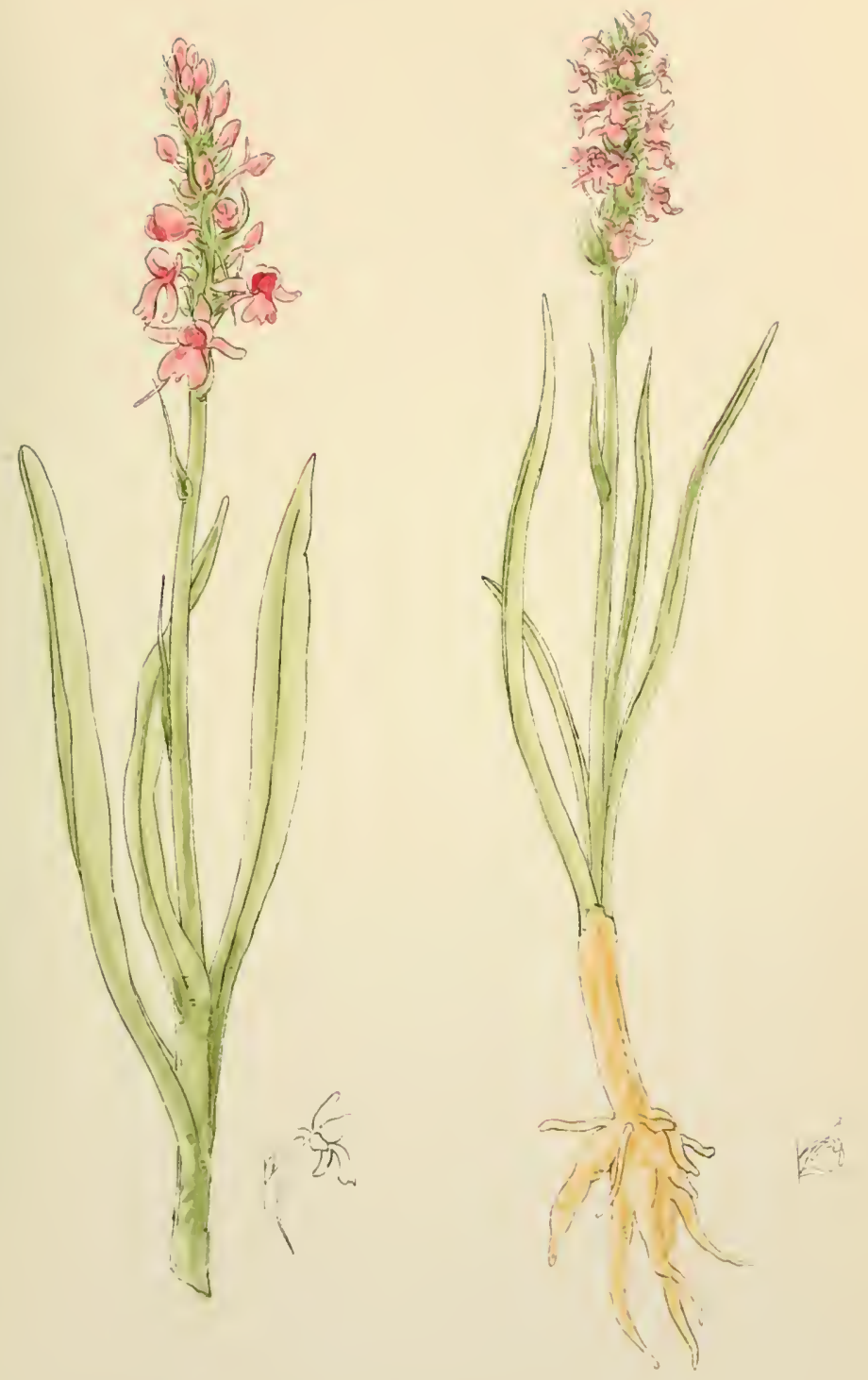

Gymnadenia conopsea.

Gymnadenia odoratissima. 

ccVIl.
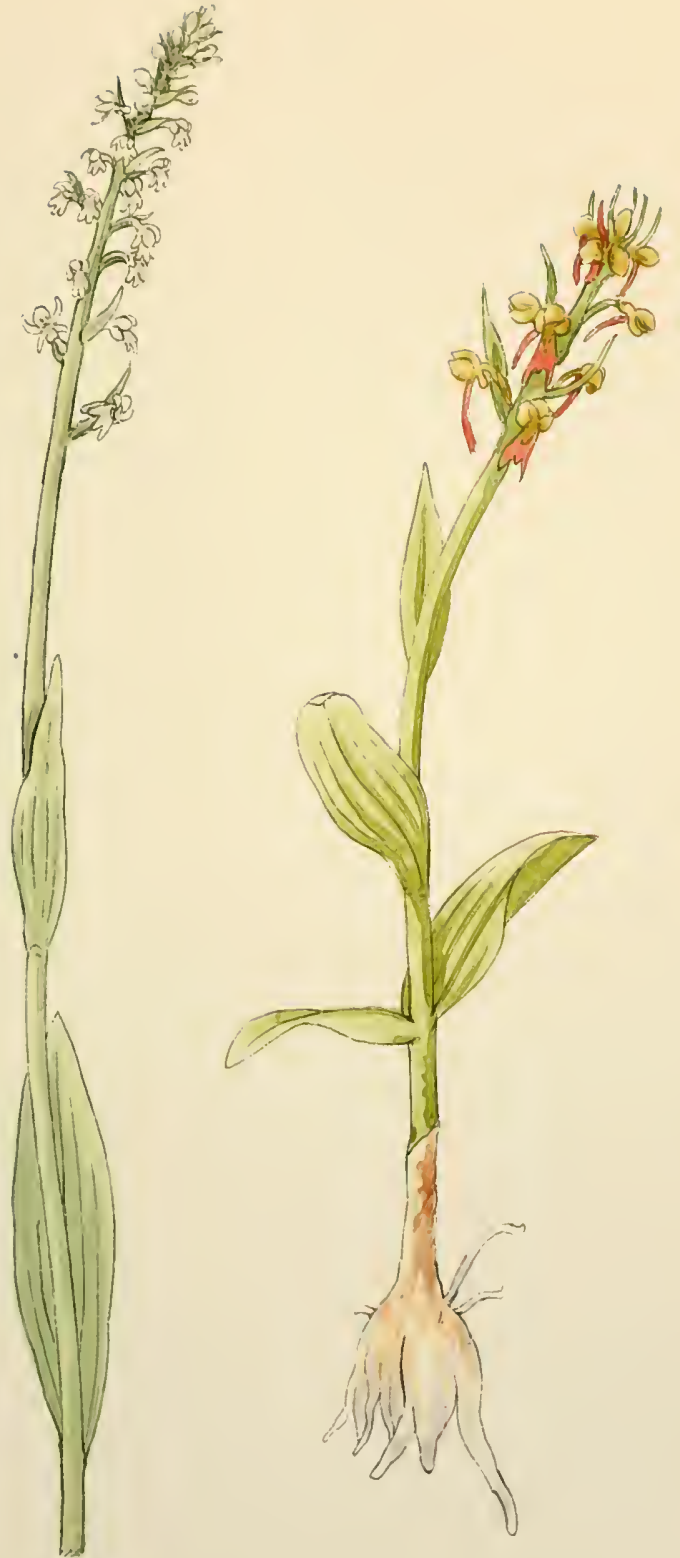

Gymnadenia albida.

Habenaria viridis. 



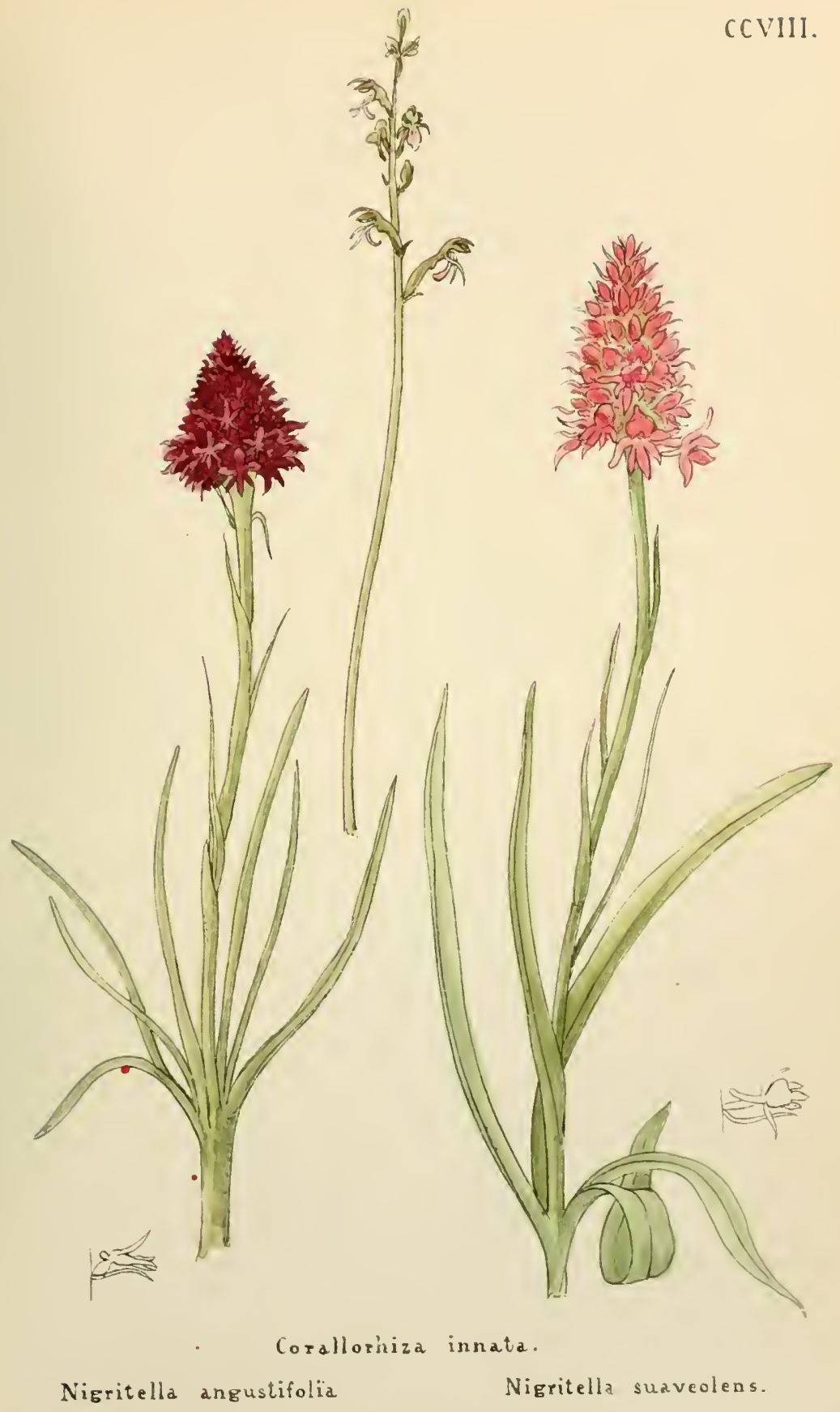



ccix. 

CCX.
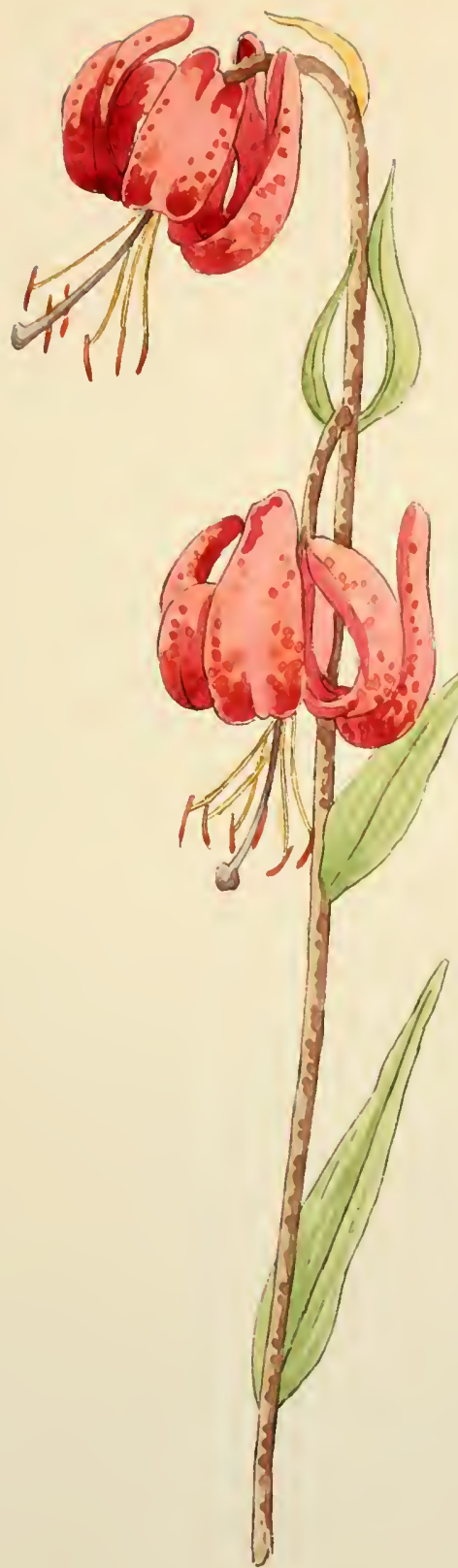

Lilium Martagon. 


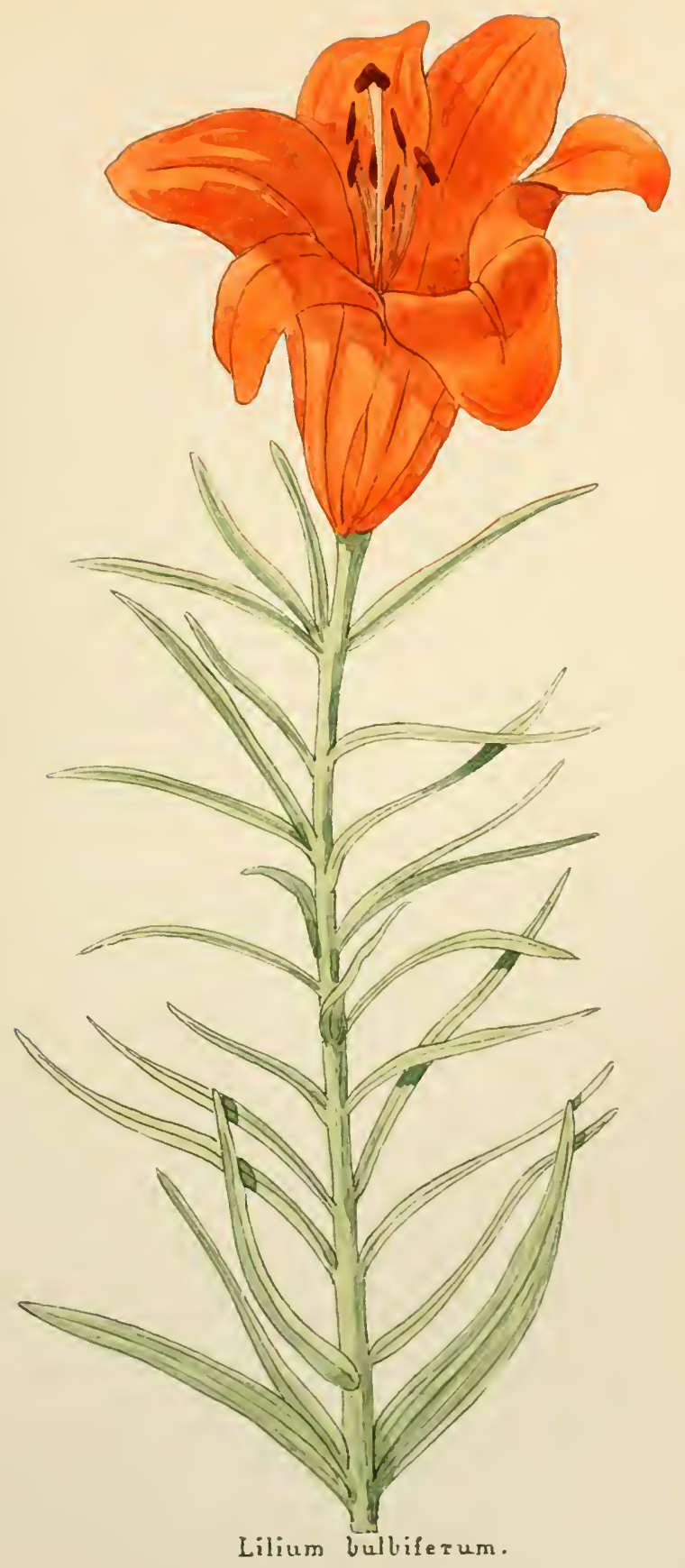

CCXI. 

CCXII.
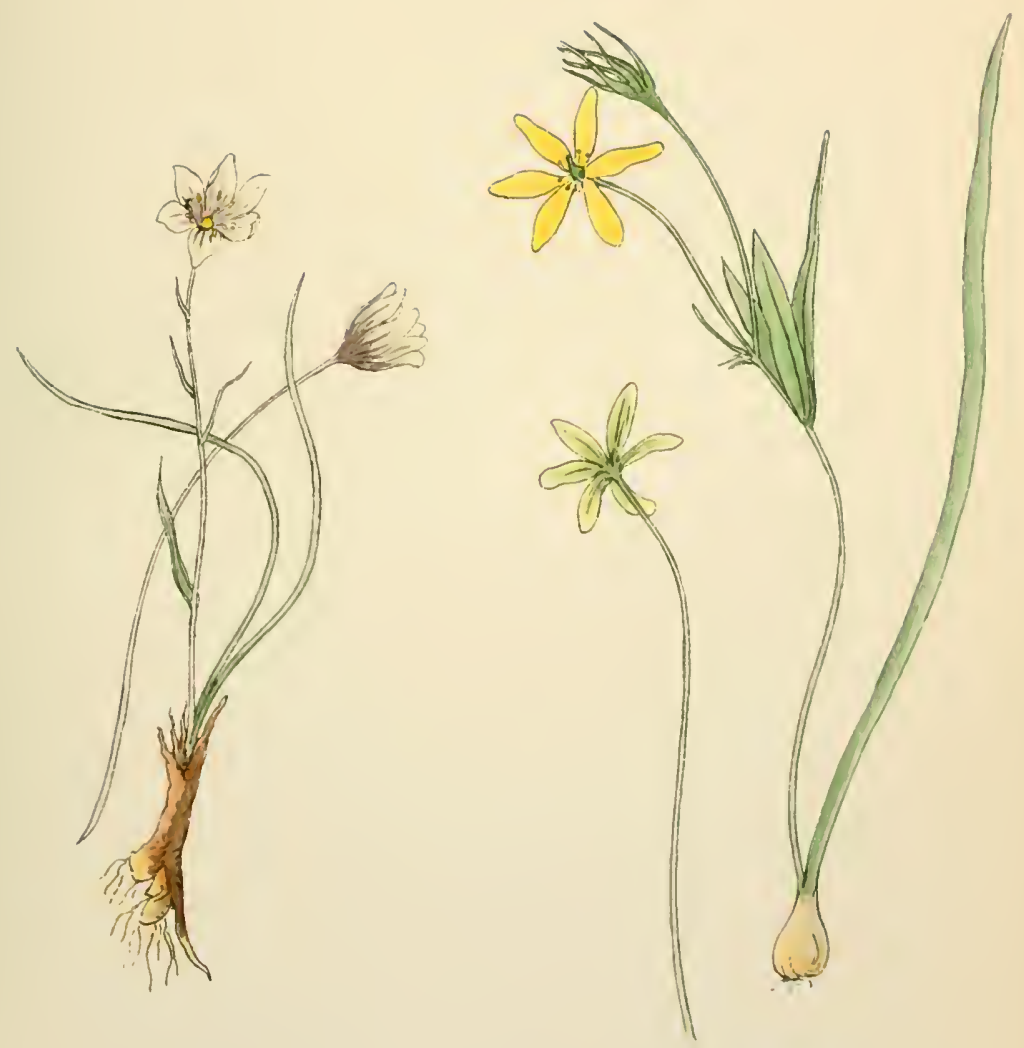

Lloydia serotina.

vagea Livitardi. 



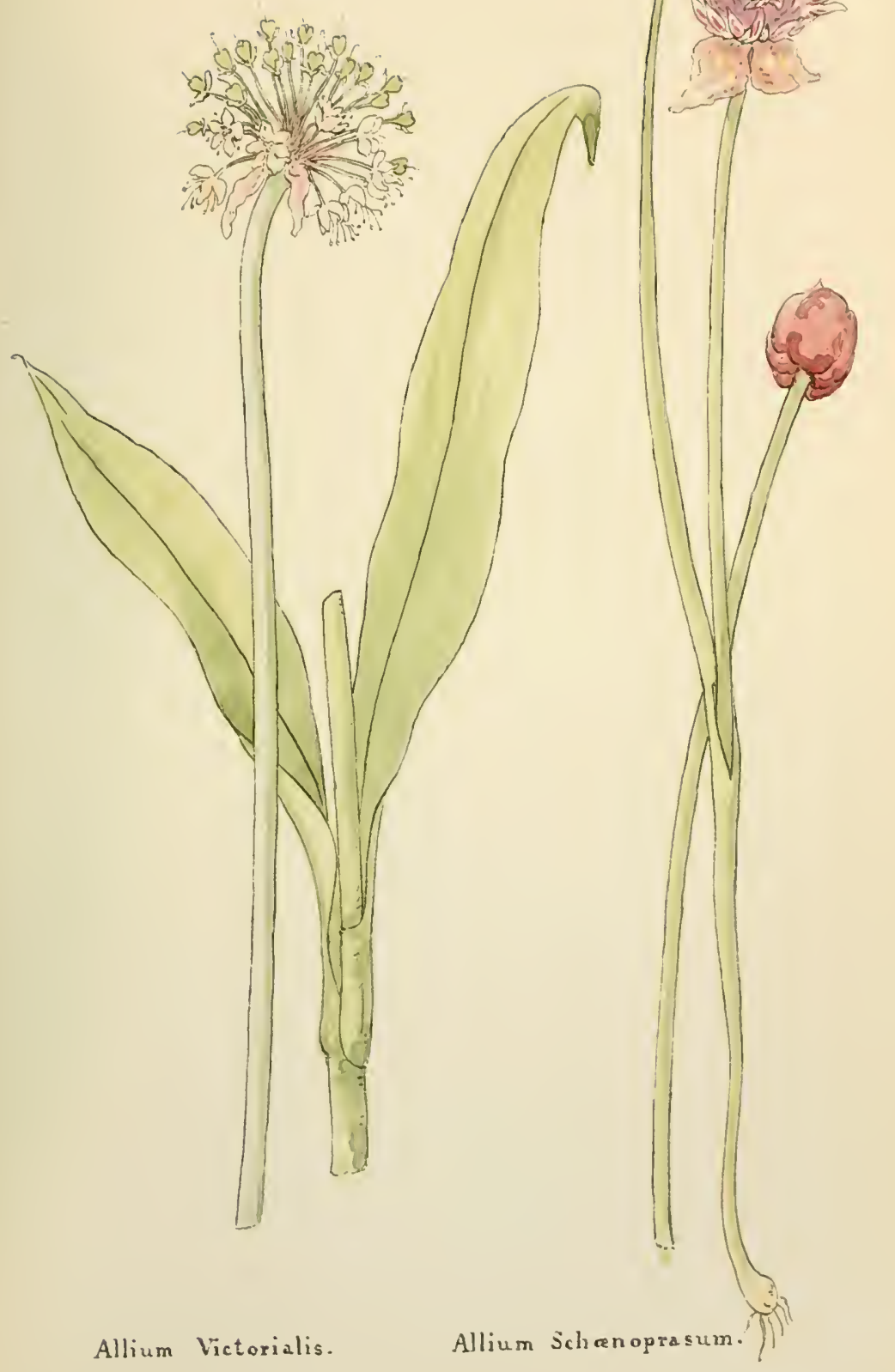





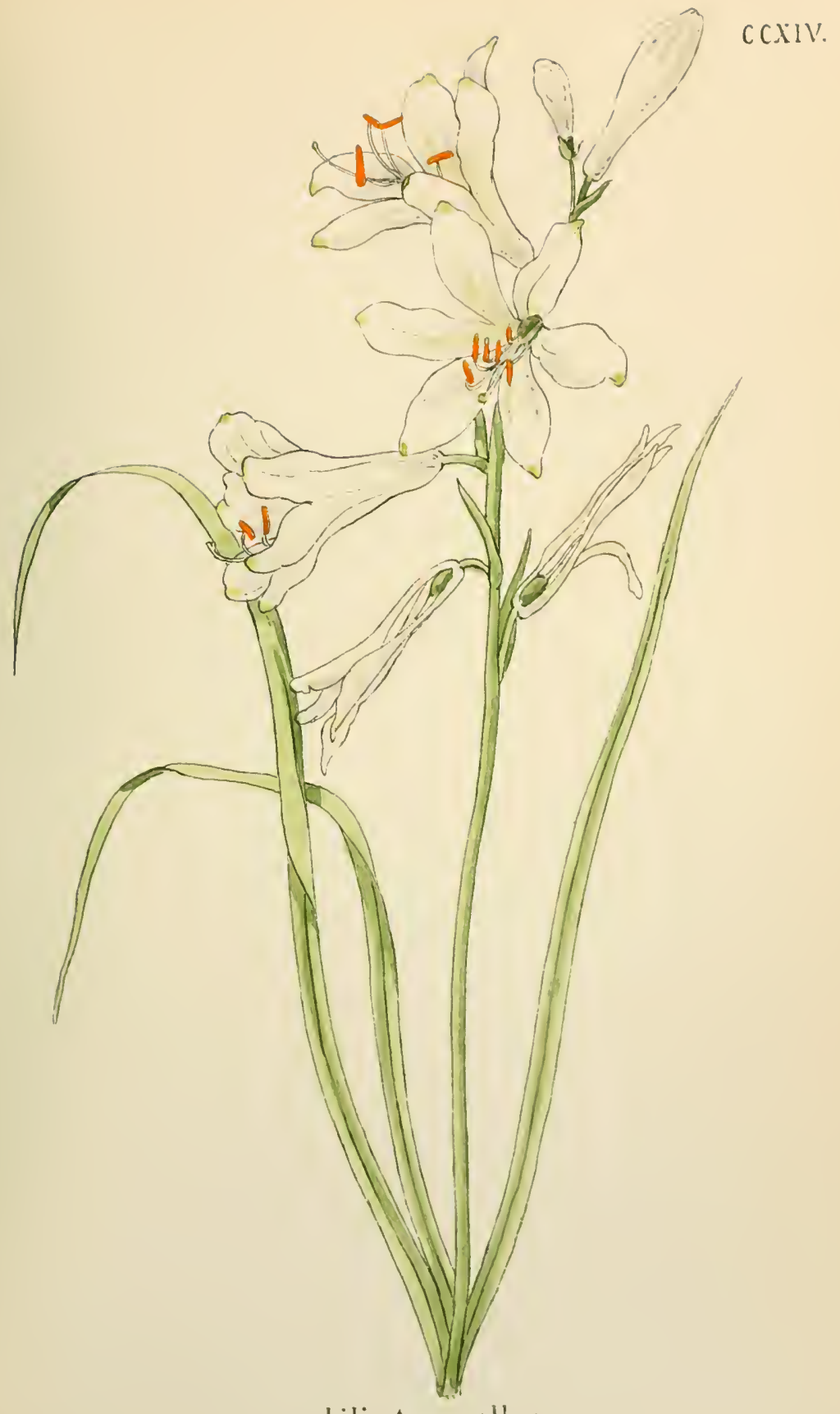

Liliastrum allum. 

CCXV.

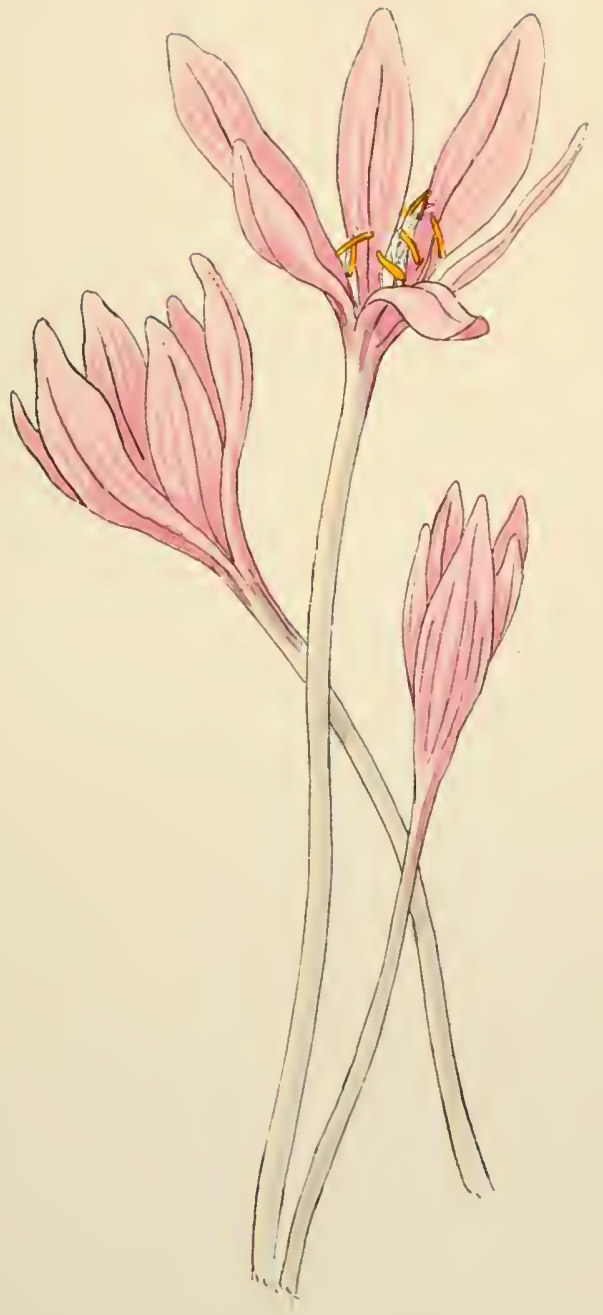

Colchicum autumnale. 

CCXVI.
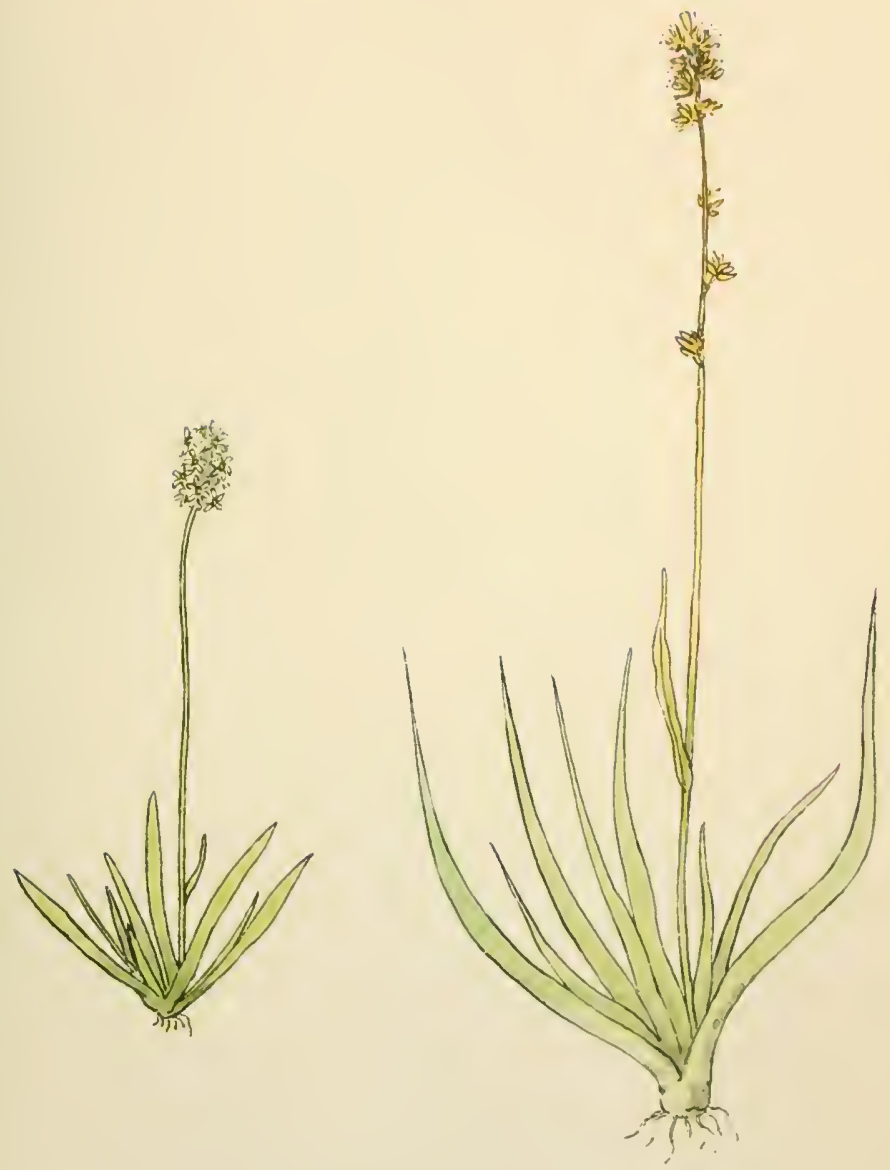

Tofieldia palustris.

Tofieldia calyculata. 


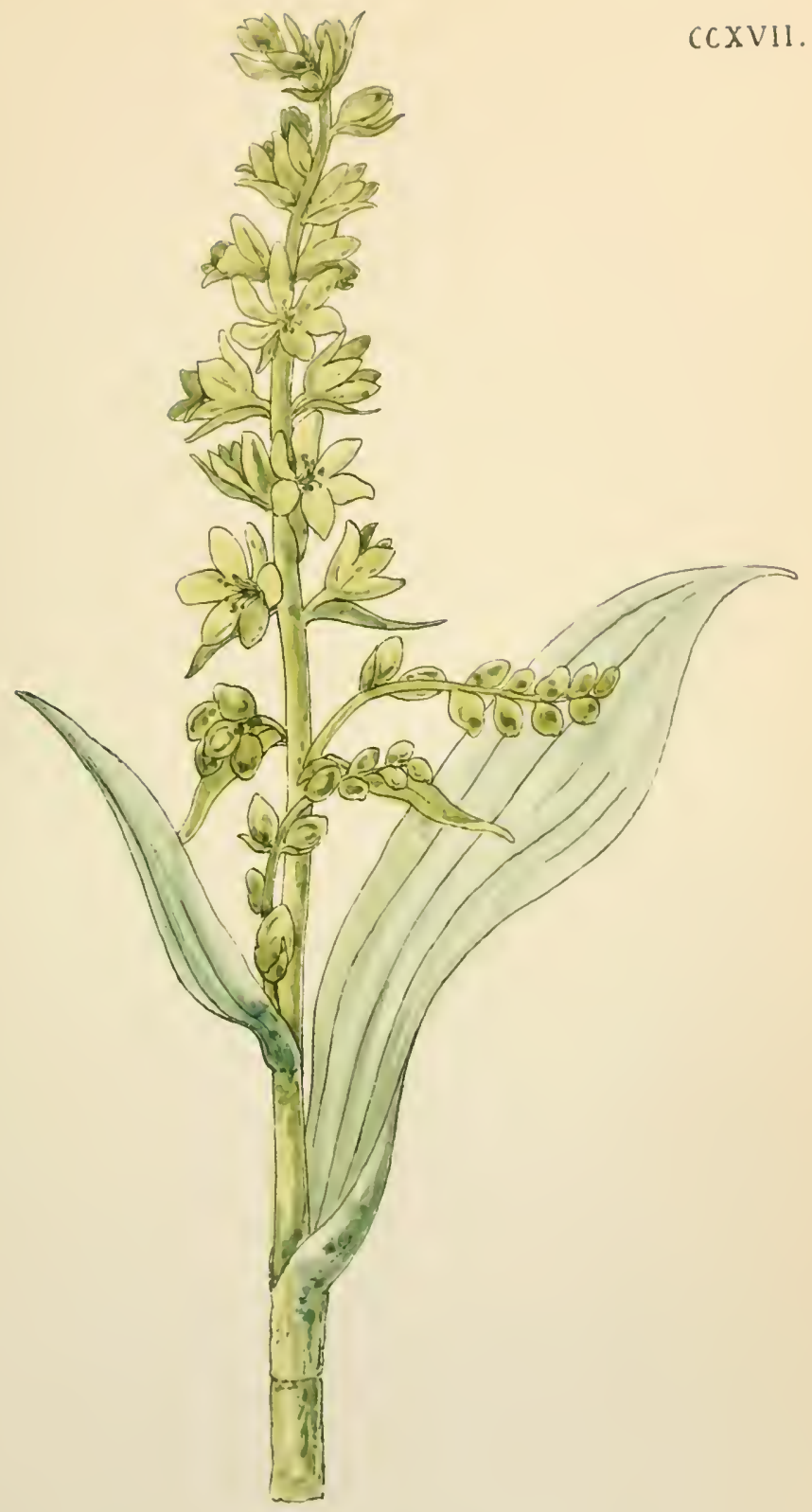

Veratrum allum. 



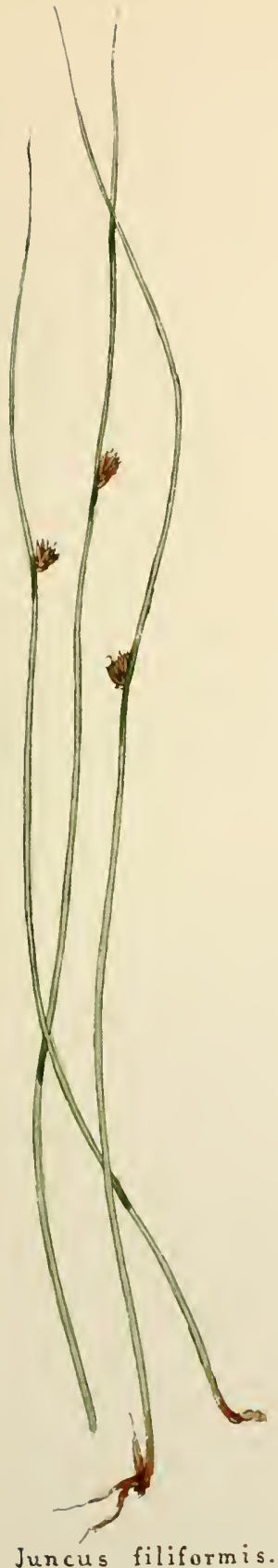

CCXVill .

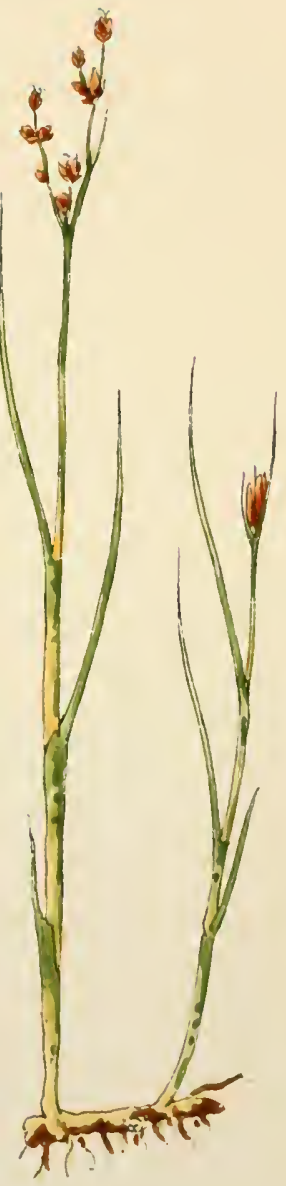

Juncus filiformis.

Juncus alpinus. 

C XIX.
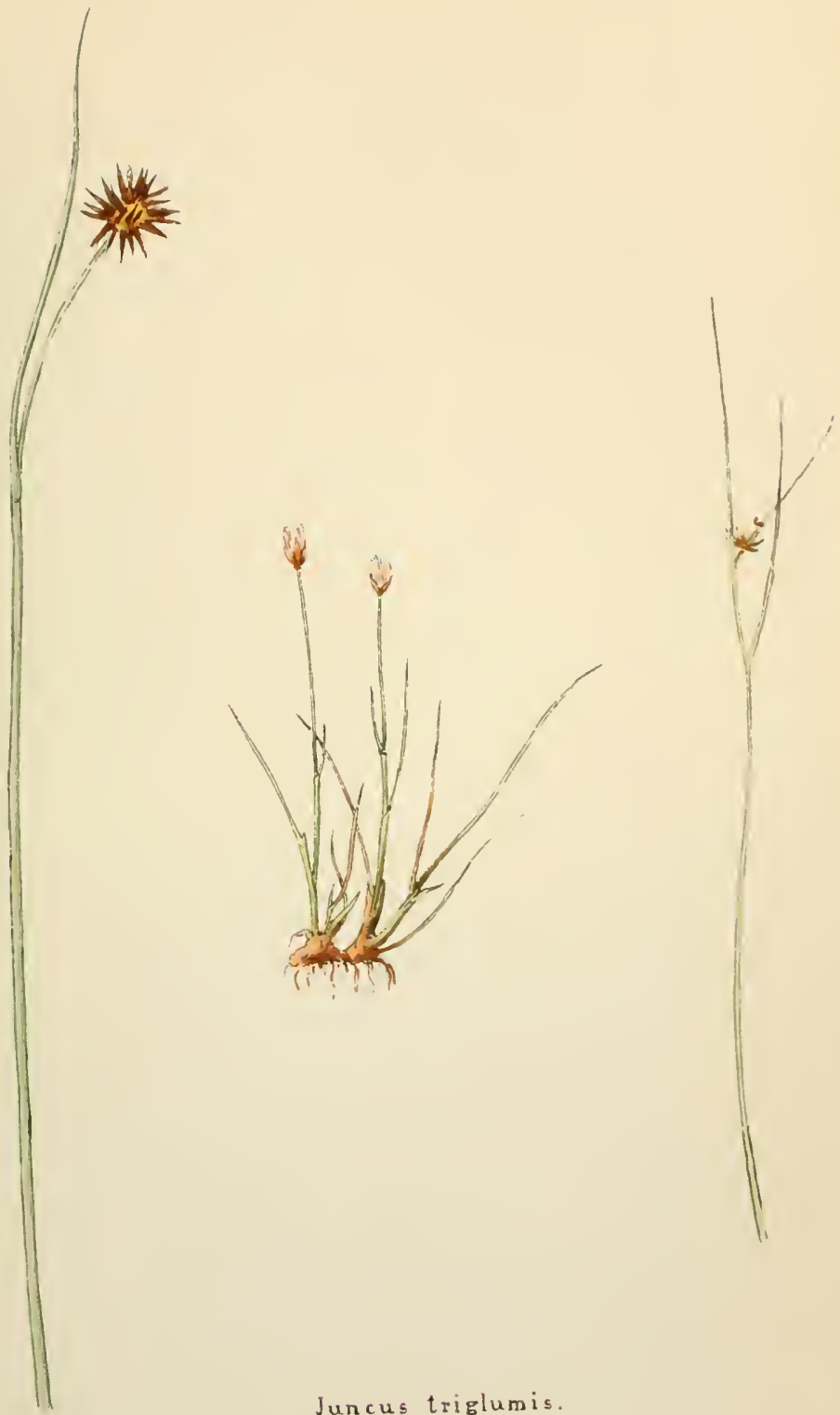

Juncus triglumis.

Juncus Jacquini.

Juncus trifidus. 

CCXX.
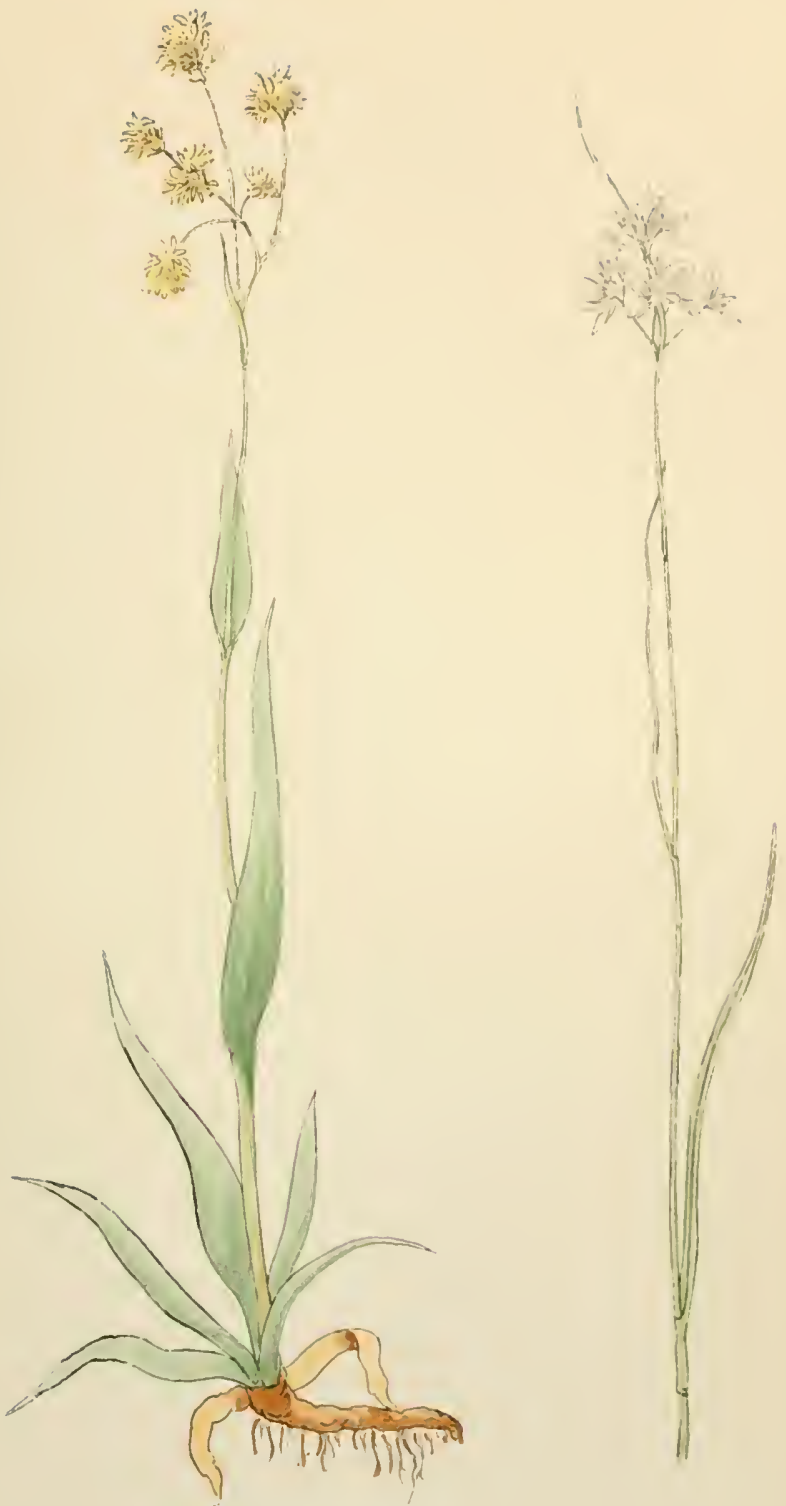

Luzula lutea. 



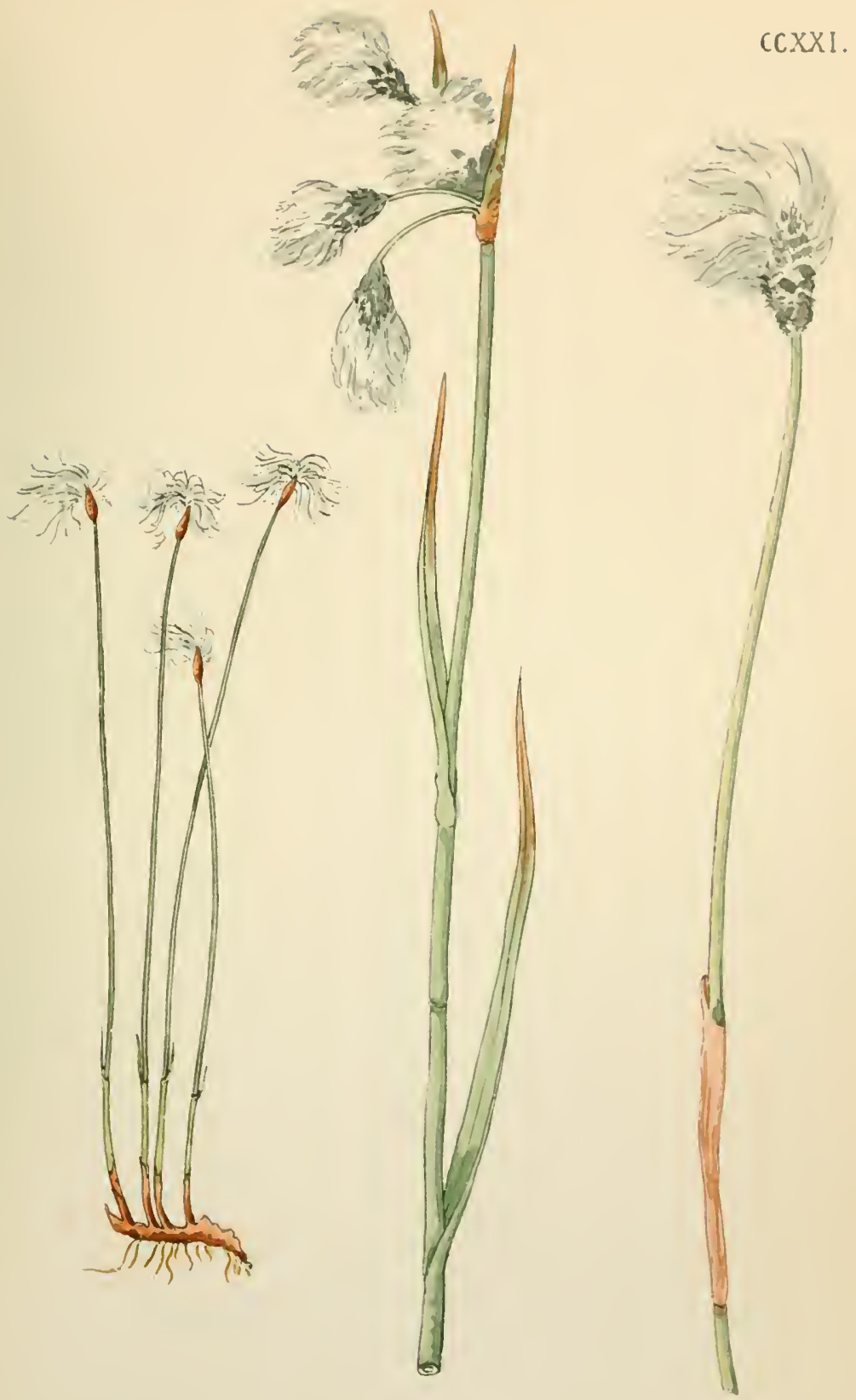

Eriophorum alpinum. Eriophorum angustifolium. Eriephorum Scheuchzeri. 



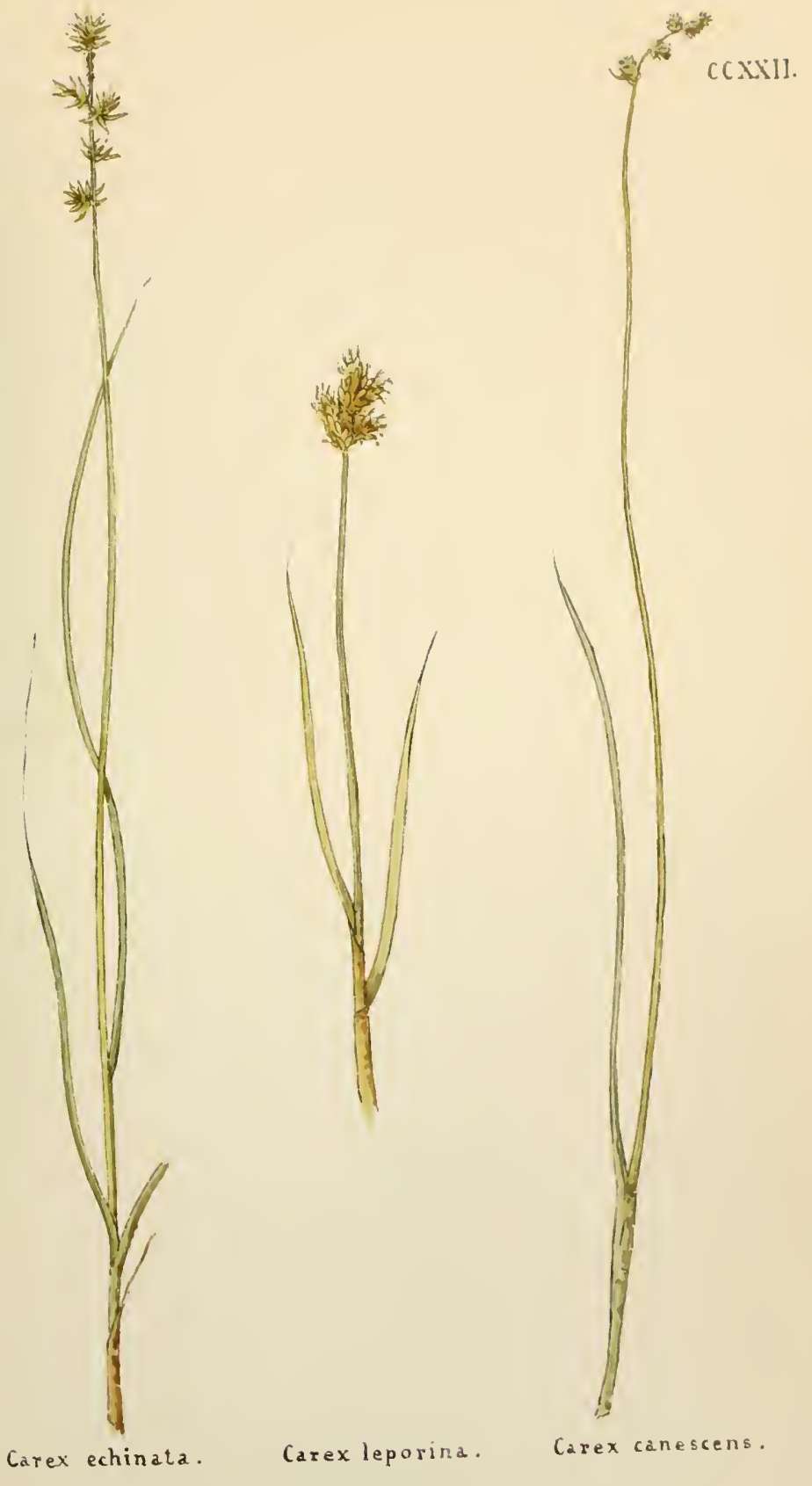


CXXII].

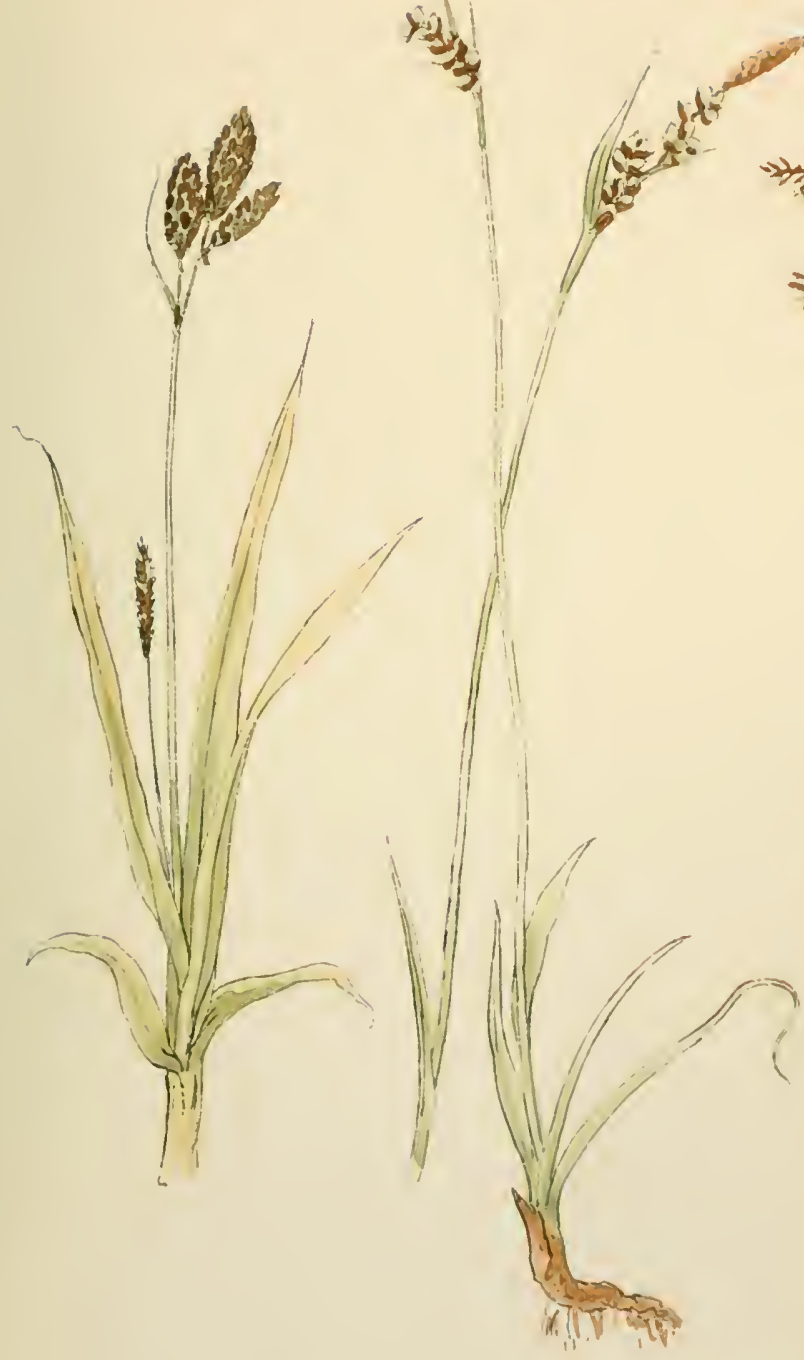

?
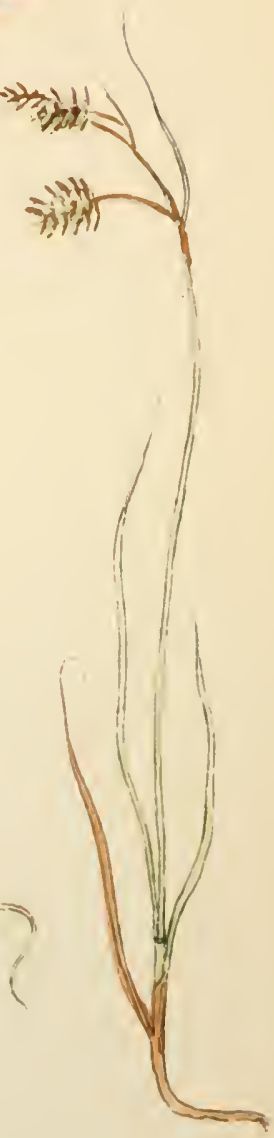

Carex nigra.

Cartex panicea.

Carex limosa. 



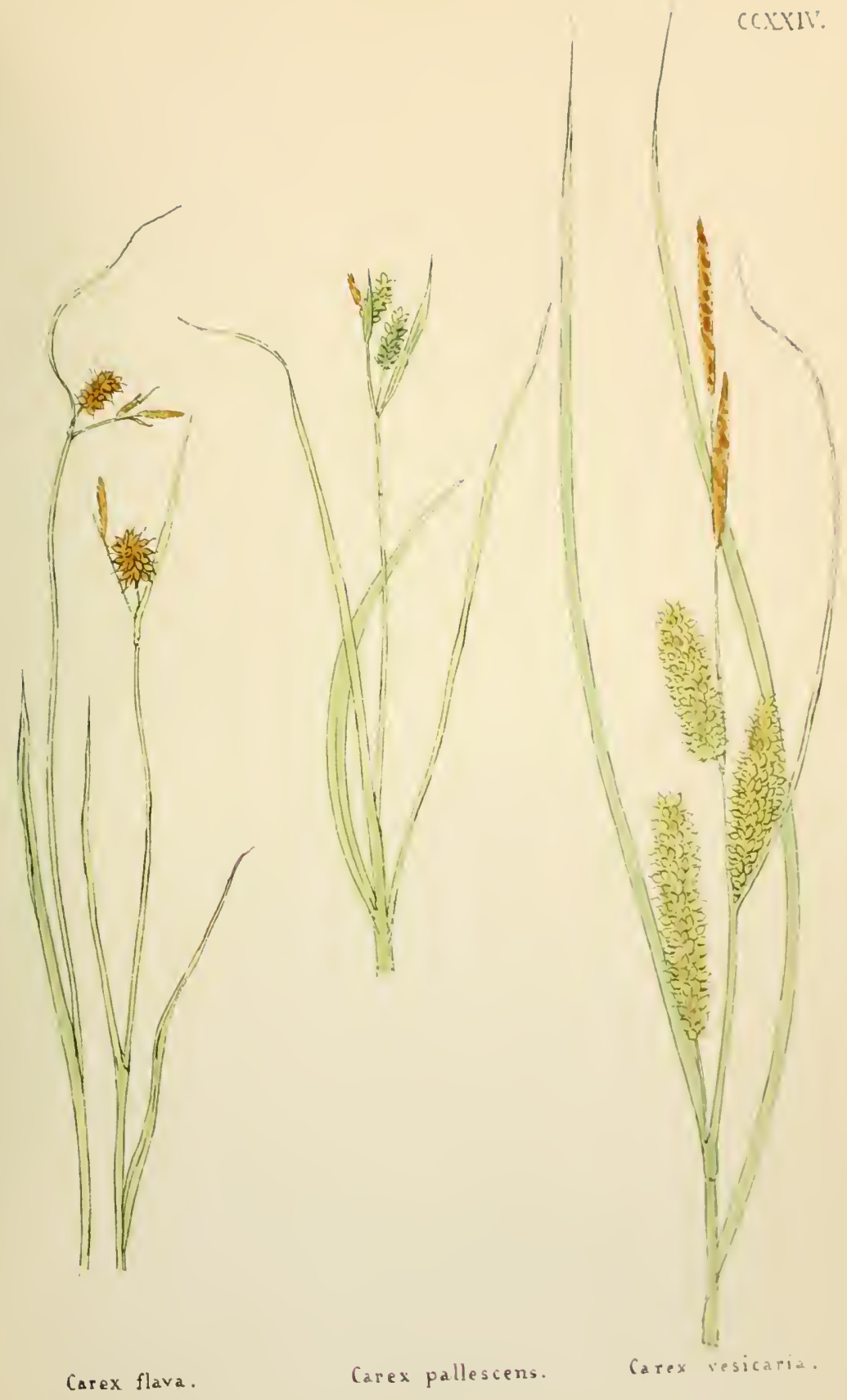










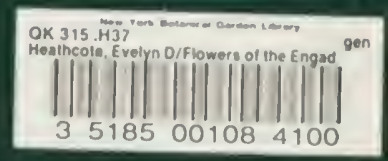




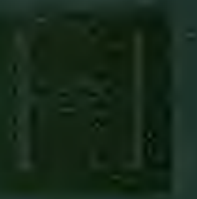

c 